\title{
Large intermediate-depth earthquakes and the subduction process
}

\author{
Luciana Astiz ${ }^{1}$, Thorne Lay ${ }^{2}$ and Hiroo Kanamori ${ }^{1}$ \\ ' Seismological Laboratory, California Institute of Technology, Pasadena, CA (U.S.A.) \\ ${ }^{2}$ Department of Geological Sciences, University of Michigan, Ann Arbor, MI (U.S.A.)
}

(Received September 22, 1987; accepted October 21, 1987)

\begin{abstract}
Astiz, L., Lay, T. and Kanamori, H., 1988. Large intermediate-depth earthquakes and the subduction process. Phys.
\end{abstract} Earth Planet. Inter., 53: 80-166.

This study provides an overview of intermediate-depth earthquake phenomena, placing emphasis on the larger, tectonically significant events, and exploring the relation of intermediate-depth earthquakes to shallower seismicity. Especially, we examine whether intermediate-depth events reflect the state of interplate coupling at subduction zones, and whether this activity exhibits temporal changes associated with the occurrence of large underthrusting earthquakes. Historic record of large intraplate earthquakes $\left(m_{\mathrm{B}} \geq 7.0\right)$ in this century shows that the New Hebrides and Tonga subduction zones have the largest number of large intraplate events. Regions associated with bends in the subducted lithosphere also have many large events (e.g. Altiplano and New Ireland). We compiled a catalog of focal mechanisms for events that occurred between 1960 and 1984 with $M>6$ and depth between 40 and $200 \mathrm{~km}$. The final catalog includes 335 events with 47 new focal mechanisms, and is probably complete for earthquakes with $m_{B} \geq 6.5$. For events with $M \geq 6.5$, nearly $48 \%$ of the events had no aftershocks and only $15 \%$ of the events had more than five aftershocks within one week of the mainshock. Events with more than ten aftershocks are located in regions associated with bends in the subducted slab. Focal mechanism solutions for intermediate-depth earthquakes with $M>6.8$ can be grouped into four categories: (1) Normal-fault events (44\%), and (2) reverse-fault events (33\%), both with a strike nearly parallel to the trench axis. (3) Normal or reverse-fault events with a strike significantly oblique to the trench axis $(10 \%)$, and (4) tear-faulting events (13\%). The focal mechanisms of type 1 events occur mainly along strongly or moderately coupled subduction zones where a down-dip extensional stress prevails in a gently dipping plate. In contrast, along decoupled subduction zones great normal-fault earthquakes occur at shallow depths (e.g., the 1977 Sumbawa earthquake in Indonesia). Type 2 events, with strike subparallel to the subduction zone, and most of them with a near vertical tension axis, occur mainly in regions that have partially coupled or uncoupled subduction zones and the observed continuous seismicity is deeper than $300 \mathrm{~km}$. The increased dip of the downgoing slab associated with weakly coupled subduction zones and the weight of the slab may be responsible for the near vertical tensional stress at intermediate depth and, consequently, the change in focal mechanism from type 1 to type 2 events. Events of type 3 occur where the trench axis bends sharply causing horizontal (parallel to the trench strike) extensional or compressional intraplate stress. Type 4 are hinge-faulting events. For strongly coupled zones we observed temporal changes of intermediate-depth earthquake activity associated with the occurrence of a large underthrusting event. After the occurrence of a large underthrusting event, the stress axis orientation of intermediate-depth earthquakes changes from down-dip tensional to down-dip compressional (e.g., 1960 Chile, 1974 Peru, 1982 Tonga and 1952 Kamchatka earthquakes), or the number of large intermediate events decreases for a few years (e.g., 1964 Alaska and 1985 Valparaiso earthquakes). We conclude that even though the stress changes induced by slab pull and slab distortion control the general pattern of intermediate-depth seismicity, spatial and temporal variations of the intraplate stress associated with interplate coupling are important in controlling the global occurrence of large intermediate-depth events.

\section{Introduction}

In the theory of plate tectonics, subduction of oceanic lithosphere plays a primary role, with the contact zone between subducting and overriding plates being the locus of large underthrusting earthquakes. Such large interplate thrust earthquakes have been extensively studied, and are 


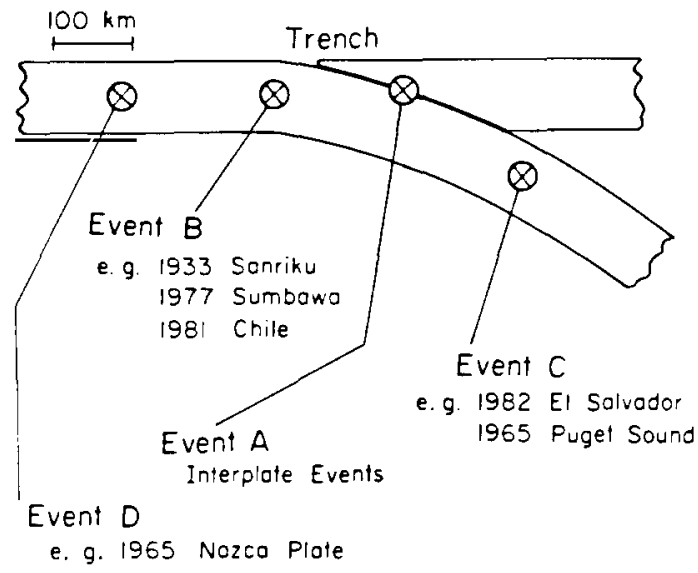

Fig. 1. A schematic figure showing the location of the seismic events near a subduction boundary. Event A: thrust events on the interplate boundary. Event $B$ : trench and outer-rise events. Event C: intermediate-depth events occurring in the subducted plate. Event D: oceanic intraplate events. Also indicated are examples of events for some of these categories.

basically well understood within the context of the tectonic convergence process. However, while most large earthquakes in subduction zones involve interplate thrusting (event $\mathrm{A}$ in Fig. 1), a significant number are intraplate events within the subducting lithosphere. These intraplate ruptures occur as shallow events near the trench axis (e.g., 1933 Sanriku, 1977 Sumba) or in the outer-rise region (e.g., 1981 Chile, event B in Fig. 1), or they occur as deeper events located down-dip from the plate interface within the subducting slab (e.g., $1982 \mathrm{El}$ Salvador, event $\mathrm{C}$ in Fig. 1). Intraplate events also occur well removed from subduction zones, in the interior of oceanic plates (e.g., $1965 \mathrm{Nazca}$, event $\mathrm{D}$ in Fig. 1). In general, our understanding of the process producing the intraplate activity is very limited, principally because the tectonic context of intraplate events is much more difficult to interpret than that of interplate events.

In order to improve our understanding of the nature of intermediate-depth seismicity, this paper presents a review of the spatio-temporal distribution of large $(M \geq 7)$ intermediate and deep focus earthquakes that occurred during this century. Given our relative ignorance of the causes of intermediate-depth events, as well as their hazard potential, we also conduct a global investigation of the focal mechanisms of recent large intermediate-depth events. We explore the interaction between the shallow seismically coupled region, where thrust events occur, and the deeper uncoupled region in subduction zones. This analysis was motivated in part by the recent suggestion that outer-rise intraplate events may, under some conditions, respond to variations in interplate coupling (Christensen and Ruff, 1983). A comprehensive catalog of focal mechanisms of large $(M>6)$ intermediate-depth earthquakes that occurred between 1960 and 1984 was gathered as our primary data base. This is used in the development of qualitative models relating gross tectonic properties to the intraplate earthquake activity. Achieving an understanding of the relationship between interplate and intermediate-depth seismic activity is particularly important for seismic gap regions and for zones of uncertain seismic potential such as the Juan de Fuca plate, where large intermediate-depth earthquakes have occurred recently.

We divide this paper into several relatively self-contained sections. Section 2 is a general review of intermediate and deep seismicity. Section 3 concentrates on the spatio-temporal distribution of large intermediate and deep focus earthquakes that occurred during this century. Section 4 presents the catalog of focal mechanisms of recent large and moderate intermediate-depth events but focuses on the relation of the larger events to the interplate seismic coupling. Section 5 studies the aftershock characteristics of intermediate-depth events with $M \geq 6.5$ in the catalog. Section 6 shows the stress-axis distribution of intermediatedepth events whereas section 7 is a regional presentation of all the events in the catalog and their relation to the recent large thrust earthquakes. Section 8 presents the discussion and conclusions.

\section{Review}

To provide a context for the analysis of the intraplate activity we will briefly review our understanding of shallow interplate earthquake phenomena. After recognizing a systematic variation in the size of interplate earthquakes in the north- 


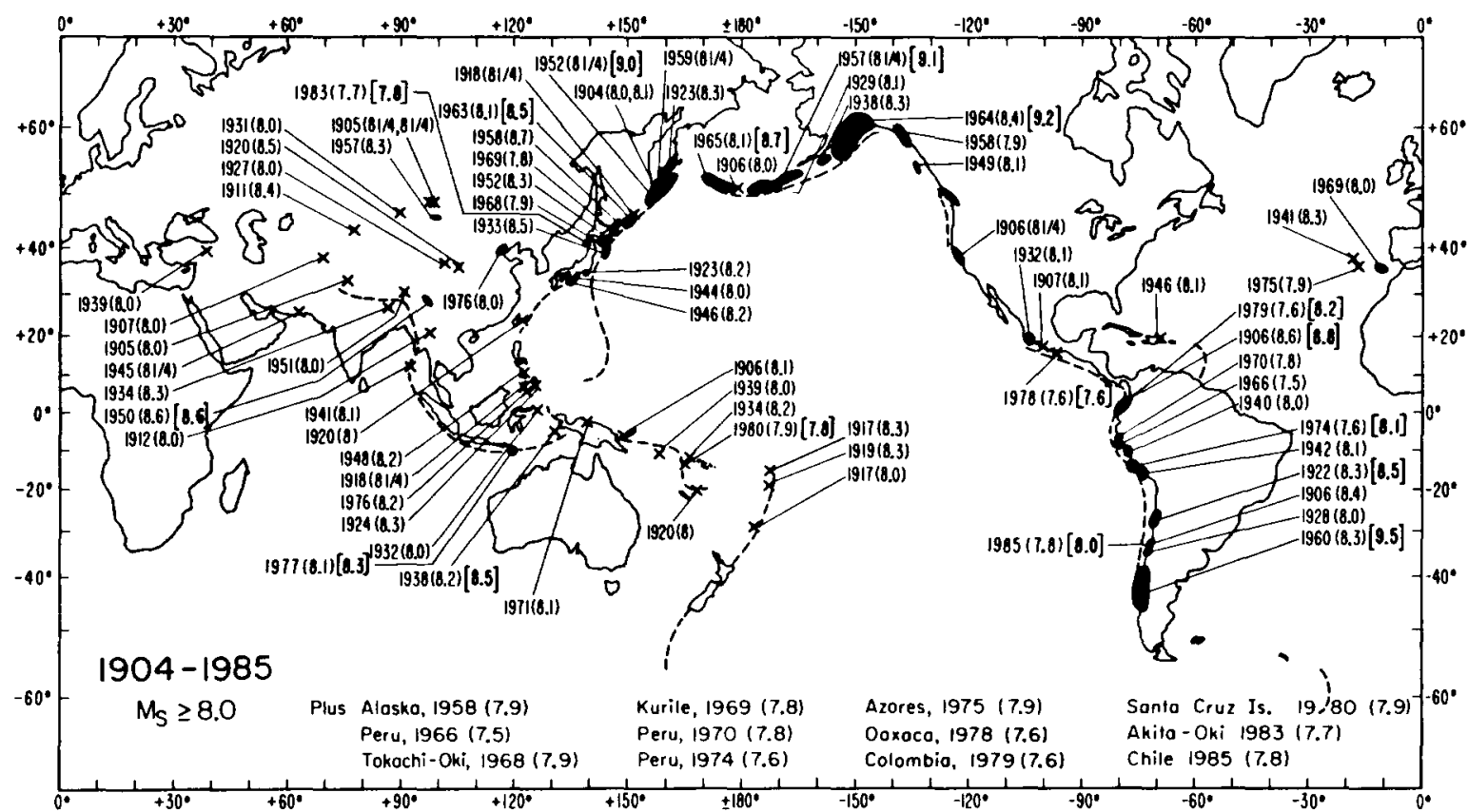

Fig. 2. Location of large $\left(M_{\mathrm{S}} \geq 7.6\right)$ shallow earthquakes from 1904 to 1985. $M_{\mathrm{S}}$ is in parentheses and $M_{\mathrm{W}}$ in brackets. Aftershock areas of recent great earthquakes are shaded. Note the uneven distribution of great earthquakes $\left(M_{\mathrm{W}}=8.5\right)$, suggesting a global variation of interplate coupling at subduction zones (from Kanamori, 1986).

west Pacific, Kanamori (1977b) introduced the term seismic coupling to describe the interaction between the two plates in subduction zones. He noticed that in some regions, such as Chile and Alaska, great earthquakes occur along the entire trench, indicating strong coupling, whereas in the Mariana Islands there is no record of great events, indicating seismic decoupling. Similarly, Kelleher et al. (1974) observed a correlation between earthquake size and width of the contact zone between the two plates at shallow depth in subduction zones. Figure 2 shows the epicenters of earthquakes with $M_{\mathrm{S}}>8.0$ that occurred between 1904 and 1985, with known aftershock areas being shaded. $M_{\mathrm{S}}$ is given in parentheses and $M_{\mathrm{W}}$ in brackets. $M_{\mathrm{w}}$ is a measure of seismic energy (Kanamori, 1977a), which remains unsaturated even for great earthquakes. Note the uneven distribution of great $\left(M_{\mathrm{W}}=8.5\right)$ earthquakes in Fig. 2 that, together with estimates of seismic and aseismic slip (Kanamori, 1977b; Sykes and Quittmeyer, 1981), suggest a global variation of seismic coupling at plate boundaries.
In an attempt to understand the subduction process, several investigators (e.g., Isacks et al., 1968; Vlaar and Wortel, 1976; Molnar et al., 1979) have studied the interrelation among the physical and geometric characteristics of subduction zones (Table I). For example, Uyeda and Kanamori (1979) characterized subduction zones by whether or not active back-arc spreading is taking place. They constructed an evolutionary model based on the degree of interplate coupling, where the end members are the Chile and Mariana type subduction zones that are respectively under regional compressional and regional tensional tectonic stresses at shallow depth. Ruff and Kanamori (1983) found a strong correlation between the maximum earthquake size observed in subduction zones and the convergence rate and age of the subducting plate; great subduction earthquakes occur where young oceanic lithosphere is being subducted at high convergence rate, while smaller events are associated with old plates with slow convergence rates. The above observations suggest that the physical characteristics of the contact 
zone between the subducted and overriding plates can be related to the degree of seismic coupling. Recently, Jarrard (1986) presented an extensive review of the relations among subduction parameters, again concluding that interplate coupling is mainly regulated by convergence rate and slab age. In summary, strongly coupled plates produce great earthquakes like the 1960 Chile $\left(M_{\mathrm{W}}=9.5\right)$, 1964 Alaska $\left(M_{\mathrm{W}}=9.1\right)$ and 1952 Kamchatka $\left(M_{\mathrm{W}}=9.0\right)$ earthquakes. Weakly coupled zones do not produce large earthquakes, as is the case for the Mariana, Bonin and Philippine arcs. Moderately coupled zones do produce large earthquakes, but with maximum ruptures $<500 \mathrm{~km}$ long, as is true for the Peru, Middle America, Aleutian and Kurile trenches (Fig. 2).

Most earthquakes occurring below $40 \mathrm{~km}$ depth near convergent plate margins are located within the subducting plate. These events form inclined zones of seismicity known as Wadati-Benioff zones, that presumably delineate the location of the colder subducting lithosphere to as deep as $650 \mathrm{~km}$ depth (e.g., Isacks et al., 1968; Sleep, 1973; Richter, 1979). It is generally believed that intraplate subduction zone events are infrequent and less important than interplate thrust events for evaluating regional seismic hazard. However, recent studies by several investigators (e.g., Abe, 1972a, b; Malgrange et al., 1981) indicate that intermediate-depth events occur frequently in some subduction zones, and are often very damaging (e.g., 1965 Puget Sound, Washington, 1973 Orizaba, Mexico; 1979 Colombia earthquakes). It is clearly a difficult task to appraise the seismic hazard posed by intraplate activity since we cannot even reliably assume that long-term plate motions will drive repeated failure on the same fault; in other words we cannot rely on the concept of seismic gaps which has been successful for forecasting interplate behavior. However, the intraplate events must be controlled by the regional stress environment, thus many studies have attempted to extract information about the state of stress within the subducting plate by analyzing intraplate focal mechanisms.

Most analyses of deep and intermediate-depth earthquakes have focused on understanding the stress distribution within the subducting litho- sphere by relating the orientation of the compressional and tensional axes of earthquake focal mechanisms to the orientation of the seismic zone. Based on a global study of individual focal mechanism solutions of deep and intermediate-depth earthquakes, Isacks and Molnar $(1969,1971)$ concluded that the descending slab acts like a stressguide, in which compressional stresses are dominant below $300 \mathrm{~km}$, and either down-dip tensional or compressional stresses are observed between 70 and $300 \mathrm{~km}$ depth. They attributed down-dip tensional events to extensional stresses induced by the slab's own negative buoyancy, but they also argue that the slab encounters more resistant material as it sinks into the mantle so that the stresses at intermediate depth become compressional for plates that extend to $650 \mathrm{~km}$ depth. Oike (1971) made similar observations by contouring pressure and tension axes for events in various subduction zones. Moment tensor inversions of recent earthquakes (Vassiliou, 1984) also indicate that down-dip compressional stresses are dominant below $300 \mathrm{~km}$ depth, while the behavior of intermediate-depth events is much more complicated, as discussed below.

Richter (1979) suggested that the occurrence of down-dip compressional events below $300 \mathrm{~km}$ is evidence of the inability of the subducted material to penetrate below $700 \mathrm{~km}$ depth. Vassiliou et al. (1984) conducted numerical modeling to test whether the observed stress pattern within the subducted slab (transition from down-dip tension to compression with increasing depth) is the result of the slab encountering a penetrable viscosity increase or an impenetrable chemical discontinuity in the mantle. Either hypothesis can explain the gross characteristics of the stress orientation. Vassiliou et al. (1984) further suggested that the observed decrease in seismic activity between 250 and $400 \mathrm{~km}$ depth may be the result of the olivine to spinel phase transition at $400 \mathrm{~km}$ depth (Anderson, 1967) and that the lack of seismicity below $670 \mathrm{~km}$ depth indicates that either the slab cannot penetrate below this depth or that a change of the subducted lithosphere rheology occurs, which somehow prevents brittle earthquake failure.

The non-uniform stress pattern observed at intermediate depths may be related to the existence 
TABLE I

Characteristics of subduction zones

\begin{tabular}{|c|c|c|c|c|c|c|c|c|c|}
\hline \multirow{2}{*}{$\begin{array}{l}\text { Region } \\
\text { SP-OP }\end{array}$} & \multirow{2}{*}{$\begin{array}{l}\text { Subduction } \\
\text { Zone Name }\end{array}$} & \multirow{2}{*}{$\begin{array}{c}\text { Age } \\
\text { (m.y.) }\end{array}$} & \multirow{2}{*}{$\begin{array}{l}\text { Dip } \\
(\mathrm{deg}) \\
\end{array}$} & \multirow{2}{*}{$\begin{array}{l}\text { TAz } \\
(\mathrm{deg}) \\
\end{array}$} & \multirow{2}{*}{$\begin{array}{l}\text { TLg } \\
(\mathrm{km}) \\
\end{array}$} & \multirow{2}{*}{$\begin{array}{c}\text { Depth } \\
(\mathrm{km}) \\
\end{array}$} & \multicolumn{3}{|c|}{ Plate Convergence } \\
\hline & & & & & & & $\begin{array}{c}\text { Rate } \\
(\mathrm{cm} / \mathrm{y})\end{array}$ & $\begin{array}{c}\text { Azimuth } \\
(\mathrm{deg})\end{array}$ & $\begin{array}{c}\mathrm{V}_{\mathrm{N}} \\
(\mathrm{cm} / \mathrm{y})\end{array}$ \\
\hline \multicolumn{10}{|c|}{ South America } \\
\hline \multirow[t]{7}{*}{ NA-SA } & 1-Colombia & $<20$ & $35^{1}$ & 30 & 800 & 250 & 7.9 & 81 & 6.2 \\
\hline & 2-Ecuador & 20 & $30^{1}$ & 0 & 600 & 200 & 8.0 & 82 & 7.9 \\
\hline & 3-Peru & 35 & $8^{1}$ & 335 & 1000 & 220 & 8.7 & 82 & 8.3 \\
\hline & 4-Altiplano & 50 & $28^{1}$ & 325 & 750 & 300 & 9.2 & 80 & 8.3 \\
\hline & 5-North Chile & 40 & $30^{1}$ & 2 & 720 & 300 & 9.3 & 80 & 9.1 \\
\hline & 6-Central Chile & 35 & $10^{1}$ & 10 & 600 & 200 & 9.1 & 81 & 8.6 \\
\hline & 7-South Chile & $<20$ & $27^{1}$ & 8 & 1250 & 160 & 9.0 & 81 & 8.6 \\
\hline SA-Sw & 8-Scotia & 70 & $70^{2}$ & 135 & 500 & 200 & $5.4^{3}$ & 260 & 4.4 \\
\hline \multicolumn{10}{|c|}{ Middle America } \\
\hline Ri-NA & -Rivera & $2-10$ & $10^{4}$ & 305 & 300 & 90 & $2.3^{26}$ & 39 & 2.1 \\
\hline Co-NA & 9-Mexico & $20-40$ & $15^{4}$ & 300 & 900 & 200 & 7.0 & 37 & 6.9 \\
\hline $\mathrm{Co}-\mathrm{Cb}$ & 10-C. America & $35-45$ & $60^{5}$ & 314 & 1500 & 280 & 8.0 & 34 & 7.8 \\
\hline \multicolumn{10}{|c|}{ Caribbean } \\
\hline $\mathrm{Cb}-\mathrm{NA}$ & 11-Greater Antilles & $125 ?$ & $75^{\circ}$ & 85 & 300 & 180 & 0.2 & NS? & 0.2 \\
\hline $\mathrm{NA}-\mathrm{Cb}$ & 12-Lesser Antilles & 80 & $65^{7}$ & 150 & 800 & 250 & 2.0 & 282 & 1.5 \\
\hline \multicolumn{10}{|c|}{ North Pacific } \\
\hline JF-NA & 13-Juan de Fuca & 10 & $22^{8}$ & 350 & 500 & 100 & $3.8^{\circ}$ & 45 & 3.1 \\
\hline \multirow[t]{2}{*}{ Pc-NA } & 14-Alaska & 45 & $45^{10}$ & 210 & 2000 & 160 & 7.2 & 329 & 6.3 \\
\hline & 15-Aleutians & 60 & $63^{10}$ & 260 & 3000 & 280 & 8.0 & 320 & 6.9 \\
\hline \multicolumn{10}{|c|}{ Western Pacific } \\
\hline \multirow[t]{4}{*}{ Pc-EU } & 16-Kamchatka & 90 & $50^{10}$ & 216 & 450 & 540 & 8.8 & 305 & 8.8 \\
\hline & 17-North Kuriles & 95 & $50^{10}$ & 216 & 500 & 600 & 9.0 & 298 & 8.9 \\
\hline & 17-South Kuriles & 100 & $45^{10}$ & 230 & 500 & 625 & 9.2 & 298 & 8.5 \\
\hline & 18-NE Japan & 100 & $40^{11}$ & 236 & 900 & 580 & 9.4 & 294 & 8.0 \\
\hline \multirow[t]{3}{*}{$\mathrm{Pc}-\mathrm{Ph}$} & 19-Izu-Bonin & 135 & $45^{12}$ & 165 & 1000 & 560 & $6.5^{13}$ & 278 & 6.0 \\
\hline & 20-North Marianas & $>150$ & $80^{14}$ & 135 & 350 & 640 & $4.7^{13}$ & 295 & 4.1 \\
\hline & 20-South Marianas & $>150$ & $80^{14}$ & 235 & 400 & 640 & $3.5^{13}$ & 305 & 3.3 \\
\hline \multicolumn{10}{|c|}{ Philippine Sea } \\
\hline Ph-EU & -Nankai & $9-20$ & $35^{15}$ & 250 & 800 & 60 & $4.0^{13}$ & 310 & 3.5 \\
\hline & 21-Ryukyu & 45 & $65^{12}$ & 210 & 1000 & 220 & $5.6^{18}$ & 310 & 5.5 \\
\hline & 22-North Taiwan & 65 & $70^{12}$ & 20 & $120 ?$ & 300 & $6.0^{13}$ & 130 & 5.6 \\
\hline EU-Ph & 23-Luzon & $20-35$ & $60^{18}$ & 5 & 500 & 640 & $7.3^{13}$ & 130 & 5.2 \\
\hline $\mathrm{Ph}-\mathrm{EU}$ & 24-Philippines & 55 & $60^{18}$ & 165 & 1500 & 640 & $8.3^{13}$ & 295 & 6.4 \\
\hline EU-? & 25-Sulawesi & $60 ?$ & $62^{15}$ & 188 & 350 & 190 & - & NS? & $?$ \\
\hline Indone & & & & & & & & & \\
\hline In-EU & 26-Burma & - & $60^{17}$ & 0 & 900 & 200 & 6.0 & 20 & 2.1 \\
\hline In-EU & 27-Andaman & 55 & $50^{18}$ & 335 & 1000 & 100 & 6.3 & 21 & 4.5 \\
\hline & 28-Sunda & 80 & $47^{18}$ & 320 & 1500 & 300 & 7.0 & 25 & 6.3 \\
\hline & 29 Java & 135 & $60^{18}$ & 280 & 1700 & 650 & 7.7 & 22 & 7.5 \\
\hline & 30-Timor & 90 & $70^{20}$ & 255 & 1100 & 690 & 7.8 & 20 & 7.7 \\
\hline Southv & st Pacific & & & & & & & & \\
\hline $\mathrm{Bi}-\mathrm{In}$ & 31-New Guinea & $30 ?$ & $55^{21}$ & 118 & 550 & 200 & $3.3^{22}$ & 203 & 3.3 \\
\hline So-Bi & 32-New Britain & $30 ?$ & $60^{21}$ & 254 & 500 & 200 & $9.2^{22}$ & 343 & 9.2 \\
\hline So-Pc & 33-New Ireland & $30 ?$ & $75^{21}$ & 300 & 400 & 550 & $10.1^{22}$ & 34 & 10.1 \\
\hline In-Pc & 34-Solomon & 60 & $50^{21}$ & 300 & 1000 & 200 & 10.0 & 79 & 7.7 \\
\hline & 35-New Hebrides & 65 & $70^{23}$ & 348 & 1500 & 320 & 10.0 & 85 & 9.9 \\
\hline Pc-In & 36-Tonga & 100 & $55^{24}$ & 200 & 1500 & 650 & 9.2 & 280 & 9.1 \\
\hline & 37-Kermadec & 100 & $70^{24}$ & 200 & 600 & 600 & 7.2 & 278 & 7.1 \\
\hline & 38-New Zealand & 90 & $67^{25}$ & 200 & 500 & 350 & 5.0 & 275 & 48 \\
\hline
\end{tabular}


of double seismic zones, which are observed in several regions. Well-located hypocenters in the Kanto district in Japan (Tsumura, 1973) demonstrate the existence of a double Benioff zone. Composite focal mechanisms of small events in the region indicate that the upper seismic zone is under in-plate compression whereas the lower layer is under in-plate tensional stresses (Hasegawa and Umino, 1978). The two layers of seismicity are separated by $\sim 35 \mathrm{~km}$ and extend in depth from 60 to $190 \mathrm{~km}$. Double seismic zones have been observed beneath most of the Japanese arc (e.g., Suzuki and Motoya, 1978; Yoshii, 1979; Ishikawa, 1985 ) and also in the Kuriles-Kamchatka subduction zone (Sykes, 1966; Fedotov, 1968; Fedotov et al., 1971; Veith, 1974, 1977; Stauder and Maulchin, 1976). However, double seismic zones are apparently not found in every subduction zone (Fujita and Kanamori, 1981). Some regions exhibit stress-segmented seismic zones where regions of compressional and tensional events are in close proximity, potentially giving the impression of a double-seismic zone in cross section, as is the case for the central Aleutian arc (Engdahl and Scholz, 1977; Fujita and Kanamori, 1981). Some of the mechanisms proposed to explain double seismic zones include stresses associated with phase changes (Veith, 1974), unbending of the lithosphere (Engdahl and Scholz, 1977; Samowitz and Forsyth, 1981; Kawakatsu, 1986a, b), sagging of the subducted plate (Yoshii, 1977; Sleep, 1979), and thermo-elastic stresses (House and Jacob, 1982; Molnar et al., 1979; Goto et al., 1985).

Fujita and Kanamori (1981) interpreted published focal mechanism solutions of intermediatedepth earthquakes as a function of the slab age and convergence rate as follows. Stresses at intermediate depth in old and slow slabs are tensile and induced by the slab's own weight, whereas those in old and fast slabs are generally mixed, resulting in double seismic zones. Intermediatedepth stresses in young and slow slabs are also mixed, but instead exhibit stress-segmented zones. Young and fast slabs are tensile, specifically under South America, probably owing to continental loading. Their results represent the first attempt to interpret intermediate-depth earthquakes in the context of the global variation of interplate coupling at subduction zones, which is predominantly controlled by slab age and convergence rate (Ruff and Kanamori, 1980).

Recently Yamaoka et al. (1986) studied scaled physical models of the downgoing plate in each subduction zone. They showed that the shape of the descending lithosphere, as inferred from the observed intraplate seismicity in most regions, can be explained by modeling the lithosphere by bending an inextensible spherical shell, which shows little deformation under moderate stress. Regions with a poor fit improved greatly by tearing the spherical shell in the region where the lowest seismicity is observed. Their experiments suggest

TABLE I (footnote)

SP-OP Subducted and overriding plates names (Bi-Bismark, Cb-Caribbean, Co-Cocos, EU-Eurasian, In-Indian, JF-J. Fuca, NA-North America, Pc-Pacific, Ph-Philippine, Ri-Rivera, SA-South America, Sw-Sandwich, So-Solomon)

Age of the SP from Sclatter and Parsons, 1981; and Moore, 1982

DIP of the SP at intermediate depth

TAz Trench Azimuth from Moore, 1982

TLg Approximate Trench Length from Moore, 1982

Depth of maximum extent of continuous observed seismicity. Note that there are deep events in South America but the seismicity is not continuous

Plate Convergence (Rate, Azimuth) calculated from RM2 model of Minster and Jordan, 1978; and Chase, 1978.

$V_{\mathrm{N}}$ convergence velocity normal to the trench axis

References: 1. Barazangi and Isacks, 1976; 2. Frankel and McCann, 1979; 3. Barker, 1972; 4. Molnar and Sykes, 1969, LeFevre and McNally, 1985; 5. Dewey and Algermissen, 1974, Dean and Drake, 1978; 6. Frankel et al., 1980; 7. McCann and Sykes, $1984 ; 8$. Clowes et al., 1983; 9. Nishimura et al., 1984; 10. Enghdal et al., 1977; 11. Ishida, 1970; 12. Shiono; et al., $1980 ; 13$. Seno, $1977 ; 14$. Katsumata and Sykes, 1969; 15. Kanamori and Tsumura, 1971; 16. Cardwell et al., 1980; 17. Fitch, 1970 b; 18. Eguchi et al., $1979 ; 19$. Fitch, 1970a; 20. McCaffrey et al., 1985, Cardwell and Isacks, 1978; 21. Pascal, 1979; 22. Johnson and Molnar, 1972; 23. Pascal et al., 1978; 24. Billington, 1980, Giardini and Woodhouse, 1984; 25. Ansell and Smith, 1975; 26. Reid, 1976. 
that lateral constraint and bending of the subducted lithosphere are important factors in determining the shape and the strain concentration in local regions. Similarly, numerical models of the downgoing plate calculated by Burbach and Frohlich (1986) suggest that the subducting lithosphere is remarkably cohesive, only rarely breaking or stretching. These studies imply that downdip tensional stresses due to slab pull are dominant for most subduction zones, with the exception of the Tonga trench, where down-dip compressional stresses are observed (e.g., Isacks and Molnar, 1969, Giardini and Woodhouse, 1984).

Figure $3 \mathrm{a}$ shows the static stress distribution near the interplate boundary for the intermediatedepth and outer-rise regions. At intermediate depth the upper seismic zone is in-plate compression stress, whereas the lower seismic zone is in tension (Hasegawa et al., 1979). Note that the stresses due to bending of the lithosphere in the outer-rise region are tensional at shallow depths and compressional at larger depth (Stauder, 1968; Chapple and Forsyth, 1979; Christensen and Ruff, 1987). This simple 2-D model does not consider either lateral changes in the subduction zone such as bends in the trench axes, contortion or segmentation of the subducted lithosphere, or subduction of topographic irregularities (e.g., ridges or oceanic platforms), which can presumably change the strength or orientation of the stress field in a given region (Burbach and Frohlich, 1986).
Yet this static stress regime does not explain all intraplate activity. Mogi (1973) studied the relationship between shallow and deep seismicity in the western Pacific region and concluded that temporal changes in the seismic activity at depth associated with the occurrence of large thrust events are not fortuitous, but may indicate an integral part of the earthquake cycle. Mogi's (1973) observations suggest that the descending lithosphere may act as a viscoelastic body under gradually increasing load, rather than as a rigid elastic plate (Kawakatsu, 1986a). Seno and Pongswat (1981) and Kawakatsu and Seno (1983) defined a third seismic zone in northern Japan, and suggested that the focal mechanisms of the events in this zone change in response to stress changes on the interplate boundary. Recently, McNally et al. (1986) and González-Ruiz (1986) constructed a composite spatio-temporal cross section of shallow and intermediate-depth events that occurred along the Mexican subduction zone, which suggests that stress transfer between the shallow interplate boundary and the intraplate seismic activity takes place. Furthermore, temporal variations in the focal mechanisms of intraplate earthquakes in relation to the occurrence of large thrust earthquakes have been observed in southern Chile, both in the outer-rise region (Christensen and Ruff, 1983, 1987) and at intermediate depths (Astiz and Kanamori, 1986).

Figure $3 b$ shows a dynamic model for regions

\section{STATIC}

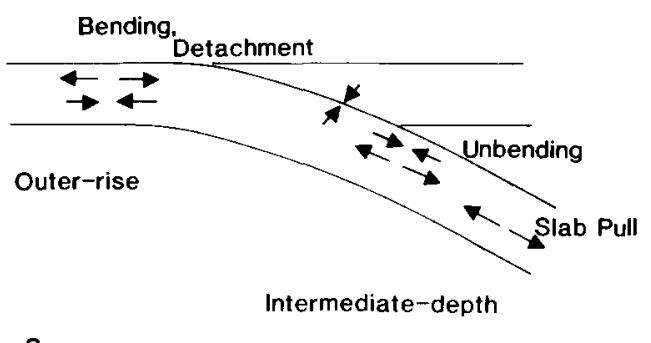

\section{DYNAMIC}

Before Thrust

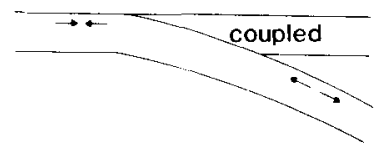

After Thrust

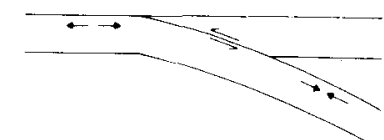

Fig. 3. (a) A 2-D model of the stress distribution within the downgoing lithosphere resulting from bending, unbending and the slab's pull in the outer-rise and intermediate-depth region. (b) A dynamic stress model that is believed to apply to strongly coupled regions near the interplate boundary. The change in the observed stress distribution is related to displacement of the interplate boundary after a large thrust event. 
in which, before a major underthrusting earthquake, the interplate boundary is strongly coupled, the down-dip slab is under tension at intermediate depths, and the outer-rise region is under compression. After displacement at the interplate boundary, the outer-rise region is under tension, whereas the down-dip slab may be either under compression or under diminished tensional stresses. Examples of observations that support this model are the temporal variation of focal mechanisms at intermediate depths in southern Chile with respect to the occurrence of the 1960 Chile $\left(M_{\mathrm{w}}=9.5\right)$ earthquake (Astiz and Kanamori, 1986) and the temporal variations of focal mechanisms in the outer-rise region in strongly coupled zones documented by Christensen and Ruff (1987). Recent elastic lithosphere models by Dmowska et al. (1987) suggest that the stress change within an earthquake cycle in the outer-rise and intermediate-depth regions would be similar to those shown in Fig. 3b.

In this study, we examine the historic record of large $\left(m_{\mathrm{B}}>7.0\right)$ intermediate and deep focus events as well as the focal mechanisms of 335 moderate and large $\left(m_{\mathrm{b}} \geq 6.0\right)$ intermediate-depth $(40-200 \mathrm{~km})$ earthquakes that occurred from 1960 to 1984 in relation to both static conditions and local variations of the strength of interplate coupling in a region. Temporal changes of focal mechanisms of intermediate-depth events owing to large thrust events are examined. We study the spatial and temporal variations for different regions and interpret them within the context of the two models in Fig. 3.

\section{Large intermediate and deep focus earthquakes: 1904-1984}

To provide a general context for a detailed investigation of recent intermediate-depth earthquakes, we first consider the historic record of the largest events within the subducting slabs. Figure 4 shows the world-wide distribution of large $(M \geq$ 7) intermediate and deep focus earthquakes that occurred from 1904 to 1984 . Most intermediate and deep focus earthquakes are located at major active plate boundaries, with only a few events

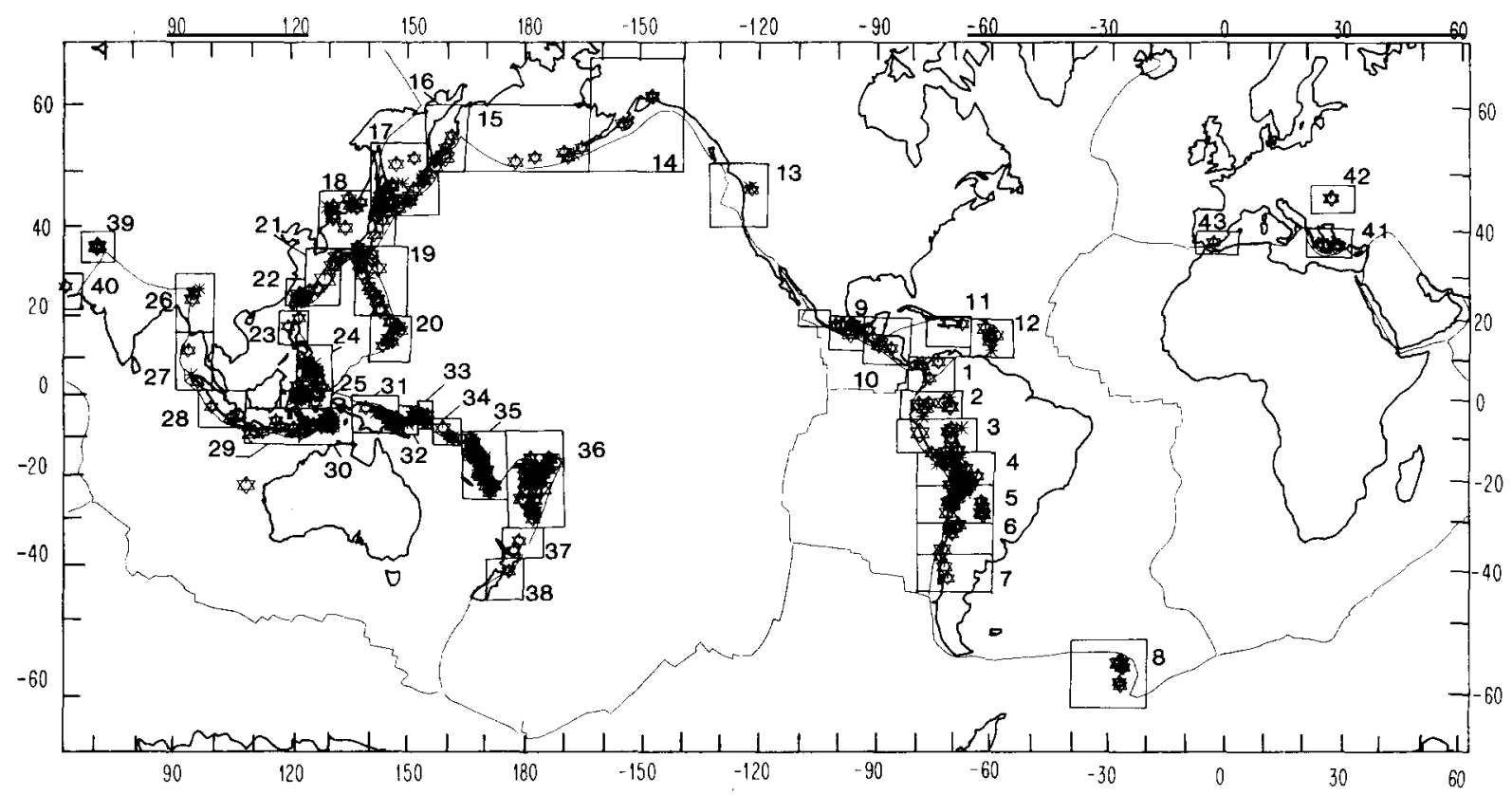

Fig. 4. Global distribution of large $(M \geq 7)$ intermediate and deep focus earthquakes that occurred between 1904 and 1984 . Symbol sizes are proportional to the earthquake magnitude, which are $m_{\mathrm{B}}$ for events that occurred before 1975 and $M_{\mathrm{W}}$ for later events. Boxes encompass regions (Table I) in the depth-magnitude profiles in Appendix I. 
apparently being unrelated to presently subducting plates. Examples of the latter include the 1954 deep Spanish event (Hodgson and Cook, 1956; Richter, 1958; Chung and Kanamori, 1976) or the 1915 event located offshore western Australia, which may be mislocated. These events are listed by region in Table A (Appendix 1). It is believed that this catalog is relatively complete for $m_{\mathrm{B}} \geq 7$ and depth greater than $70 \mathrm{~km}$, from 1904 to the present. Appendix 1 shows the depth and time distribution for each region in Fig. 4.

The most active region at intermediate depths is the New Hebrides (35). Tonga (36) is the most active below $400 \mathrm{~km}$ depth, but also has relatively abundant intermediate-depth seismic activity. Regions with large numbers of intermediate-depth earthquakes with $m_{\mathrm{B}} \geq 7.0$ are the Altiplano (4), Timor (30), Sulawesi (25), Scotia (8), and New Ireland (33), all of which have substantial distortions of the subducted lithosphere. Other regions with a large number of large intermediate-depth events are North Chile (5), Kuriles (17), Ryukyu (21), and the Philippines (24). The Nankai and Rivera regions, where the subducting lithosphere is young and the observed seismicity is all shallower than $100 \mathrm{~km}$, do not have large intraplate earthquakes. Some regions such as Luzon (23) and New Zealand (38) had very few intraplate events in this century.

By comparing the time distribution of large earthquakes in this century with the time distribution of these events after 1960 (see Appendix 1 for details), we can get an idea of how well seismicity after 1960 represents the intraplate activity of different subduction zones. Regions where seismicity within the subducting plate has been relatively constant in the last 80 years are the Altiplano (4), North Chile (5), Kuriles (17), Northeast Japan (18), Timor (30), New Hebrides (35), Tonga (36) and the Hindu-Kush (39). However, seismic activity in some other regions has changed during this century. For example the Ryukyu (21) and Sulawesi (23) regions were especially active in the early part of the century, whereas other regions such as Peru (3), Philippines (24) and New Guinea (31) seem to be more active recently. These temporal changes in activity may be related either to changes in the regional sub- duction process (e.g., the shallow interplate earthquake cycle) or to global changes such as the Chandler wobble (Abe and Kanamori, 1979).

Appendix 1 shows the location of large $\left(m_{\mathrm{b}} \geq\right.$ 7.0) intermediate and deep focus earthquakes together with large $\left(M_{\mathrm{S}} \geq 7.5\right)$ shallow events in a projection along strike with respect to time for each region in Fig. 4. Although no clear relation is observed between the shallow interplate activity and the intermediate-depth events in most regions, sequences of large intermediate and deep focus events tend to occur at periods when no large earthquakes occur at the interplate boundary (see figures in Appendix 1).

Vassiliou et al. (1984) determined the variation of the number of $m_{\mathrm{b}} \geq 5$ events between 1964 and 1980 from the NOAA catalog. They observed that for the global seismicity, the number of intraplate events decreases with depth exponentially to $\sim 300$ $\mathrm{km}$, then remains constant to $\sim 500 \mathrm{~km}$ depth, followed by an increase to $\sim 600 \mathrm{~km}$ depth. They assumed that this distribution reflects the level of stress within the plate with increasing depth. Giardini (1987) determined the $b$-value for deep seismic regions and found large regional variations, from 0.33 in South America to 1.22 in Tonga. The $b$-value for most regions is $\sim 0.6$. Chung and Kanamori (1980) investigated the variation of earthquake source parameters with depth and concluded that the stress drop of intermediate and deep events ranges from $20 \mathrm{bar}$ to $\sim 4.6 \mathrm{kbar}$, with a general increase with depth; however, events close to bends in the subducting lithosphere have higher stress drops than events at comparable depth located in undistorted subducting slabs. The exact relation between absolute stress within the plate and earthquake stress drop is unclear. However, since most of the principal seismic energy is released in large earthquakes, the profiles shown in Appendix 1 should reflect the seismic energy released within the plate for each subduction zone during this century.

We divide the large intraplate earthquakes into three depth ranges $(40 \leq d \leq 260 ; 260<d \leq 400$; and $d>400 \mathrm{~km}$ ) that roughly correspond to different stress regimes within the downgoing slab (Vassiliou et al., 1984), and assign a maximum observed earthquake magnitude, $m_{\mathrm{B}}$, to each 
TABLE II

Largest earthquake magnitude by seismic depth

\begin{tabular}{|c|c|c|c|c|c|}
\hline \multirow{2}{*}{$\begin{array}{l}\text { Subduction } \\
\text { Zone Name }\end{array}$} & \multicolumn{4}{|c|}{ Maximum Observed Magnitude } & \multirow{2}{*}{$\begin{array}{l}\text { Seismic } \\
\text { Depth } \\
(\mathrm{km})\end{array}$} \\
\hline & $\begin{array}{c}\mathrm{M}_{\mathrm{w}} \\
\mathrm{d}<40 \mathrm{~km}\end{array}$ & $\begin{array}{c}\mathrm{m}_{\mathrm{B}} \\
40<\mathrm{d} \leq 260\end{array}$ & $\begin{array}{c}m_{B} \\
260<d<400\end{array}$ & $\begin{array}{c}m_{B} \\
d>400 \mathrm{~km} \\
\end{array}$ & \\
\hline \multicolumn{6}{|l|}{$\overline{\text { South America }}$} \\
\hline 1-Colombia & 8.8 & 7.0 & & & 250 \\
\hline 2-Ecuador & $(88)$ & 7.5 & & 7.5 & 200 \\
\hline 3 -Peru & 8.2 & 7.8 & & 7.3 & 220 \\
\hline 4-Altiplano & & 7.6 & & & 300 \\
\hline 5-North Chile & $(8.5)$ & 7.7 & & 7.4 & 300 \\
\hline 6-Central Chile & 8.5 & 7.5 & & & 200 \\
\hline 7-South Chile & 9.5 & 7.5 & & & 160 \\
\hline 8-Scotia & 7.0 & 7.6 & & & 200 \\
\hline \multicolumn{6}{|l|}{ Middle America } \\
\hline 9a-Rivera & 8.2 & & & & 90 \\
\hline 9-Mexico & 8.1 & 7.7 & & & 200 \\
\hline 10-C. America & $(8.1)$ & 7.4 & & & 280 \\
\hline \multicolumn{6}{|l|}{ Caribbean } \\
\hline 11-Greater Antilles & $(8.0)$ & 7.0 & & & 180 \\
\hline 12-Lesser Antilles & 7.5 & 7.5 & & & 250 \\
\hline \multicolumn{6}{|l|}{ North Pacific } \\
\hline 13-Juan de Fuca & & 7.1 & & & 100 \\
\hline 14-Alaska & 9.2 & 7.3 & & & 160 \\
\hline 15-Aleutians & 9.1 & 7.4 & & & 280 \\
\hline \multicolumn{6}{|l|}{ North Pacific } \\
\hline 16-Kamchatka & 9.0 & 7.8 & & & 540 \\
\hline 17-Kuriles & 8.5 & 7.6 & 7.5 & 7.4 & 625 \\
\hline 18-NE Japan & 8.2 & 7.5 & 7.4 & 7.4 & 580 \\
\hline \multicolumn{6}{|l|}{ Western Pacific } \\
\hline 19-Izu-Bonin & 7.2 & 7.9 & 7.4 & 7.2 & 560 \\
\hline 20-Marianas & 7.2 & 7.3 & 7.0 & & 640 \\
\hline 21a-Nankai & $(8.2)$ & & & & 60 \\
\hline 21-Ryukyu & 8.0 & 8.1 & & & 220 \\
\hline 22-North Taiwan & & 7.6 & & & 300 \\
\hline 23-Luzon & & 7.0 & & & 640 \\
\hline 24-Philippines & $(8.3)$ & 7.3 & 74 & 7.2 & 640 \\
\hline 25-Sulawesi & $(8.0)$ & 7.8 & & & 190 \\
\hline \multicolumn{6}{|l|}{ Indonesia } \\
\hline 26-Burma & & 7.4 & & & 200 \\
\hline 27-Andaman & $(8.0)$ & 7.2 & & & 100 \\
\hline 28-Sunda & 7.9 & 7.3 & & 7.2 & 300 \\
\hline 29-Java & 7.1 & 7.6 & 7.0 & 7.2 & 650 \\
\hline 30-Timor & $(8.5)$ & 7.8 & 7.2 & 7.0 & 690 \\
\hline \multicolumn{6}{|l|}{ Southwest Pacific } \\
\hline 31-New Guinea & $(8.0)$ & 7.3 & & & 200 \\
\hline 32-New Britain & $(8.1)$ & 7.2 & & & 200 \\
\hline 33-New Ireland & & 7.3 & 7.2 & & 550 \\
\hline 34-Solomon & 8.1 & 7.3 & & & 200 \\
\hline 35-New Hebrides & 7.9 & 7.9 & 7.3 & & 320 \\
\hline 36-Tonga & 8.3 & 7.7 & 7.5 & 7.6 & 650 \\
\hline 37-Kermadec & 8.1 & 7.0 & 7.0 & & 600 \\
\hline 38-New Zealand & 7.8 & 7.1 & & & 350 \\
\hline
\end{tabular}

$M_{W}$ are mostly taken from Ruff and Kanamori (1980); values in parentheses are $M_{S}$ from Abe (1981). $\mathrm{m}_{B}$ values are body-wave magnitude at $T \approx 8$ s (Table $A$ ) 
depth range. Observe that the maximum $m_{\mathrm{B}}$ decreases with depth for most regions (Table II) with the exception of the Sunda (28), Java (29) and Tonga (36) regions, for which the observed magnitude $m_{\mathrm{B}}$ increases for the deepest range, and the Philippines (24) for which the middle range (260 $<d \leq 400 \mathrm{~km}$ ) has the largest observed $m_{\mathrm{B}}$. The deepest range $(d>400 \mathrm{~km})$ corresponds to the region with down-dip compressional events (e.g., Isacks and Molnar, 1971). In the middle depth range, down-dip tensional events predominate (Vassiliou et al., 1984) with the exception of Tonga. In the shallowest range $(40-260 \mathrm{~km})$ many events have nearly down-dip tensional axes; however, the focal mechanisms of these events exhibit a large variation as shown in the following sections. The above observations suggest that the occurrence of large intraplate events may be influenced not only by the slab's rheology but also by other factors such as lateral thermal variations in the surrounding mantle.

In an attempt to understand the regional variation in maximum earthquake size for intraplate events, we test the correlation between maximum observed $m_{\mathrm{B}}$ of the intraplate events (Table II) and the various physical characteristics of subduction zones (Table I) for the three depth ranges mentioned above as well as the classical definitions of intermediate $(70-300 \mathrm{~km})$ and deep events $(d>300 \mathrm{~km})$. The linear correlation coefficients, which vary between 0 and \pm 1 for increasing correlation, are given in Table III.

The correlations between maximum observed seismicity and plate age are small for all depth ranges. Similarly, the correlations between the depth of the continuous seismicity and the maximum size of intraplate events are small. This

\section{TABLE III}

Linear correlation coefficients

\begin{tabular}{crrll}
\hline Seismicity & \multicolumn{1}{c}{ Age } & Depth & Rate & \multicolumn{1}{l}{ Dip } \\
\hline $40 \leq d \leq 260$ & 0.12 & 0.08 & 0.17 & -0.15 \\
$260<d \leq 400$ & -0.30 & -0.14 & 0.52 & -0.70 \\
$d>400 \mathrm{~km}$ & -0.38 & -0.35 & 0.55 & -0.44 \\
$70 \leq d \leq 300$ & 0.22 & 0.15 & 0.05 & 0.14 \\
$d>300 \mathrm{~km}$ & -0.37 & -0.23 & 0.52 & -0.51 \\
\hline
\end{tabular}

suggests that the age and depth of the slab alone do not play a major role in determining the maximum size of intraplate earthquakes. The correlation coefficient between seismicity and the convergence rate at the trench increases with depth, suggesting that the rate of influx of subducted material controls to some extent the strength between compressional stress within the plate and ultimately the size of intraplate earthquakes at depth. Finally, the correlation coefficient of the slab dip and the seismicity is small at the shallower depth range ( $40-260 \mathrm{~km})$, high for the depth range $260-400 \mathrm{~km}$, and then it decreases at the deepest range. If we consider that the slab could be modeled by a bending plate, then the torque exerted by the slab's weight is larger for a shallowly dipping slab than it is for steeply dipping plate. Then the large negative value of the dip-seismicity correlation coefficient for the middle depth range suggests that the size of earthquakes at this range may be controlled by the slab's bending moment.

\section{Catalog of focal mechanisms of recent inter- mediate-depth earthquakes}

There is evidence that subducted plates are uncoupled from the overriding plates below $\sim 40$ $\mathrm{km}$ depth, where a steepening of the Benioff zone is generally observed (e.g., Engdahl, 1977; Isacks and Barazangi, 1977; Billington, 1980). This bend in the subducting plate has been related to the phase change in the oceanic crust from basalt to eclogite that initiates at $\sim 35 \mathrm{~km}$ depth, and results in an increase in the average density of the slab (Wortel, 1982; Pennington, 1983; Ruff and Kanamori, 1983; Fukao et al., 1983). Also, many large thrust events appear to have rupture zones constrained to depths $<40 \mathrm{~km}$ (e.g., Schwartz and Ruff, 1987). Thus, most earthquakes $>40 \mathrm{~km}$ depth are believed to occur within the subducting plate, since it is no longer in contact with the overriding plate. An analysis of intraplate events in the depth range $40-200 \mathrm{~km}$ should provide us with a better understanding of the interaction between the state of stress in the deeper uncoupled region and the degree of coupling of the 
interplate boundary at subduction zones. Such a study requires a detailed catalog of events that are reliably identified as being intraplate.

We gathered a catalog of intermediate-depth earthquakes. First, all earthquakes with $m_{\mathrm{B}} \geq 6.0$ that occurred from 1960 to 1984 between 40 and $200 \mathrm{~km}$ depth were identified by sorting the NOAA and ISC (International Seismic Centre) catalogs. Then, a search for published focal mechanisms for these earthquakes was undertaken. Some events have been studied by several groups of researchers. However, only the focal mechanism solution that was considered the most reliable is listed in Table IV. Reliability was determined on the basis of the most recent study (that generally referred to earlier work) or on the basis of studies in which the authors picked the first-motion and S-polarization data themselves. Although there is a considerable variation in the confidence attached to individual focal mechanisms, the general characteristics of the solutions are reliable. If the studies included epicentral relocation then we list these hypocenters in Table IV instead of the location reported by NOAA. In surveying the literature, numerous intermediate-depth events given depths $<40 \mathrm{~km}$ or missing in the NOAA and ISC listings were identified and included in Table IV. Published focal mechanism solutions were not available for nearly 140 of the earthquakes in the initial list.

A preliminary focal mechanism solution was determined from first-motion data in the ISC bulletin for those events lacking a published solution. Most of these mechanisms were not very well constrained. If the depth of the event was shallower than $60 \mathrm{~km}$, with no depth phases being reported in the ISC bulletin, and if the preliminary focal mechanism solution was relatively reliable and consistent with an interplate thrust orientation in the direction of local subduction (Table I), we assumed that the event was probably interplate, and it was removed from the initial catalog. In this way, we eliminated $\sim 30$ events. Then we picked first-motion data from short and long-period records of the World-Wide Seismograph Standard Network (WWSSN) for over a hundred events to determine better constrained focal mechanisms. Recordings for 25 events of the catalog were very small, and reliable focal mechanism could not be determined using WWSSN seismograms. Most of these events occurred in the early 1960 s and their magnitudes may have been overestimated. These events were removed from the catalog as well. After timing depth phases in short-period WWSSN records, we found that 40 events were clearly shallow, and these were not included in the intermediate-depth earthquake catalog either. Some of these events are outer-rise events, for which a comprehensive catalog has been compiled by Christensen and Ruff (1987). These events were eliminated from our catalog. Finally, we determined 40 new focal mechanism solutions of intermediate-depth earthquakes, which are shown in Appendix 2. First-motion data were insufficient to constrain both fault planes for half of these events, but we determined the depth and one of the focal mechanism parameters by modeling long-period body waves. Only a few critically oriented stations were modeled for each of these events, but generally this provided adequate resolution of the source depth and mechanism. The asterisk $(*)$ in Table IV indicates which parameter was constrained by the synthetic seismograms or by timing of $\mathrm{pP}-\mathrm{P}$ phases in short period records to determine the event depth.

The final catalog, listed in Table IV, contains 335 hypocenters, magnitudes and focal mechanism solutions of earthquakes that occurred between 40 and $200 \mathrm{~km}$ depth. Event numbers are chronological, hypocenters are given in degrees (latitude north and longitude east are positive), depth is given in $\mathrm{km}$. The magnitude $M$ listed is $m_{\mathrm{b}}$ for most events that occurred before 1977 except for the larger events for which $m_{\mathrm{B}}$ from Abe (1981) is given. For events that occurred after 1977, $M_{\mathrm{w}}$ was determined from Kanamori's (1977a) relation: $M_{\mathrm{W}}=(2 / 3) / \log M_{0}-10.73$. The magnitudes given in Table IV are intended to reflect the real size of the event, for there is a large scatter between $m_{\mathrm{b}}$ and either $M_{\mathrm{W}}$ or $m_{\mathrm{B}}$ for the intermediate-depth events in Table IV, indicating that $m_{\mathrm{b}}$ is not a very reliable measure of large $(M \geq 7)$ intermediate-depth events. Relations between $m_{\mathrm{B}}$ and seismic moment, $M_{0}$, or $M_{\mathrm{W}}$ are given by Kanamori (1983). The fault parameters, azimuth $(\phi)$, dip $(\delta)$, and rake $(\lambda)$, of one of the possible 
TABLE IV

Catalog of intermediate-depth earthquakes

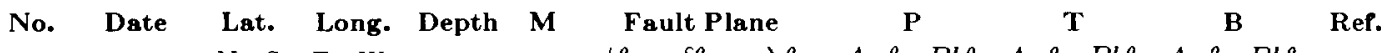
$\mathrm{N}+, \mathrm{S}-\mathrm{E}+\mathrm{W}-\quad \phi^{\circ} \quad \delta^{\circ} \quad \lambda^{\circ} \quad A z^{\circ} \mathrm{Pl}^{\circ} \quad \mathrm{Az}^{\circ} \mathrm{Pl}^{\circ} \quad \mathrm{Az}^{\circ} \mathrm{Pl}^{\circ}$

\begin{tabular}{|c|c|c|c|c|c|c|c|c|c|c|c|c|c|c|c|}
\hline 1 & $7 / 10 / 60$ & 1.00 & 98.00 & 150.0 & 6.50 & 299.0 & 16.0 & 90.0 & 209 & 29 & 29 & 61 & 119 & 0 & FK \\
\hline $1^{\prime}$ & $07 / 25 / 60$ & 54.00 & 159.00 & 100.0 & 6.90 & 340.0 & 35.0 & 41.0 & 301 & 46 & 182 & 26 & 73 & 57 & FK \\
\hline 2 & $03 / 11 / 61$ & 48.70 & 155.20 & 50.0 & 6.20 & 237.0 & 20.0 & 109.5 & 132 & & 96 & & 39 & 6 & FK \\
\hline 3 & $08 / 17 / 61$ & 46.40 & 149.30 & 160.0 & 6.70 & 271.5 & 18.7 & -25.0 & 275 & 0 & 130 & & 27 & 18 & FK \\
\hline 4 & $08 / 03 / 62$ & -23.30 & -68.10 & 108.0 & 7.30 & 142.0 & 32.8 & -123.7 & 307 & 66 & 76 & 6 & 171 & 17 & S73 \\
\hline 5 & $01 / 01 / 63$ & 56.60 & -157.70 & 50.0 & 6.50 & 156.0 & 23.5 & 58.9 & 88 & 20 & 291 & & 185 & 12 & SB \\
\hline 6 & $01 / 29 / 63$ & 49.70 & 155.00 & 143.0 & 6.20 & 159.2 & 17.5 & 42.2 & 108 & 32 & 317 & 00 & 206 & 13 & $\mathrm{SMa}$ \\
\hline 7 & $02 / 13 / 63$ & 24.50 & 122.10 & 67.0 & 7.25 & 259.0 & 29.0 & 90.0 & 169 & 16 & 349 & & 79 & 0 & KS \\
\hline 8 & $02 / 26 / 63$ & -7.60 & 146.20 & 182.0 & $7.3 \mathrm{~B}$ & 267.0 & 21.9 & -180.0 & 105 & 41 & 249 & 41 & 355 & 20 & IM \\
\hline 9 & $03 / 10 / 63$ & -29.90 & -71.20 & 70.0 & 6.30 & 82.5 & 11.5 & -158.9 & 250 & 48 & 51 & 40 & 151 & 11 & S73 \\
\hline 10 & $03 / 30 / 63$ & -19.11 & 169.00 & 156.0 & 6.30 & 278.1 & 48.9 & 13.4 & 235 & 20 & 130 & 36 & 348 & 48 & $\mathrm{IM}$ \\
\hline 11 & $04 / 02 / 63$ & 53.10 & -171.70 & 140.0 & 6.38 & 6.4 & 36.9 & -54.6 & 349 & 00 & 200 & 10 & 00 & 20 & SB \\
\hline 12 & $04 / 13 / 63$ & -6.30 & -76.70 & 125.0 & 6.10 & 20.0 & 31.2 & -63.7 & 49 & 69 & 271 & & 177 & & SB \\
\hline 13 & $05 / 01 / 63$ & -19.00 & 168.90 & 142.0 & $7.1 \mathrm{~B}$ & 296.5 & 41.4 & 23.8 & 250 & 20 & 138 & 46 & 356 & 37 & IM \\
\hline 14 & $05 / 22 / 63$ & -8.20 & 115.70 & 76.0 & 6.10 & 96.0 & 6.0 & 90.0 & 6 & 39 & 186 & 51 & 276 & 0 & F72 \\
\hline 15 & $06 / 17 / 63$ & -4.10 & 102.20 & 69.0 & 6.30 & 330.0 & 10.0 & 90.0 & 240 & 35 & 60 & 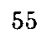 & 150 & 0 & F72 \\
\hline 16 & $06 / 19 / 63$ & 4.70 & 126.50 & 83.0 & 6.20 & 174.0 & 10.0 & 90.0 & 84 & 35 & 264 & o & 354 & 0 & F72 \\
\hline 17 & $07 / 04 / 63$ & -26.30 & -177.70 & 190.0 & 6.50 & 122.4 & 62.8 & -151.1 & 339 & 39 & 71 & 1 & 164 & 51 & ISO \\
\hline 18 & $07 / 30 / 63$ & -55.90 & -27.50 & 55.0 & 6.20 & 168.0 & 40.0 & 160.0 & 32 & 23 & 146 & 44 & 283 & 37 & F75 \\
\hline 19 & $09 / 15 / 63$ & -10.30 & 165.60 & 43.0 & 6.30 & 280.0 & 40.0 & -90.0 & 10 & 85 & 190 & 5 & 280 & 0 & JoM \\
\hline 20 & $09 / 17 / 63$ & -10.80 & -78.20 & 61.0 & 6.70 & 187.1 & 43.8 & -60.0 & 186 & 69 & 76 & 5 & 344 & 20 & S75 \\
\hline 21 & $09 / 24 / 63$ & -10.60 & -78.00 & 80.0 & 6.00 & 90.8 & 24.9 & -141.0 & 261 & 56 & 39 & 27 & 13 & 19 & S75 \\
\hline 23 & $10 / 24 / 63$ & -4.90 & 102.90 & 65.0 & 6.10 & 298.0 & 16.0 & 90.0 & 208 & 29 & 28 & 61 & 118 & 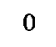 & F72 \\
\hline 24 & $11 / 04 / 63$ & -15.14 & 167.38 & 123.0 & 6.40 & 5.0 & 40.0 & 90.0 & 275 & 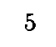 & 95 & 85 & 185 & 0 & IM \\
\hline 25 & $11 / 04 / 63$ & -6.86 & 129.58 & 100.0 & $7.8 \mathrm{~B}$ & 80.0 & 48.0 & 52.0 & 16 & 3 & 279 & & 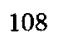 & 27 & $\mathrm{OA}$ \\
\hline 26 & $11 / 15 / 63$ & 44.30 & 149.00 & 50.0 & 6.00 & 96.5 & 14.0 & -84.6 & 179 & 59 & 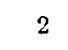 & 31 & 271 & 1 & SMa \\
\hline 27 & $12 / 18 / 63$ & -24.80 & -176.60 & 46.0 & 6.50 & 270.0 & 0.1 & -180.0 & 92 & 45 & 27 & 45 & 2. & 0 & ISO \\
\hline 28 & $01 / 15 / 64$ & 29.10 & 140.80 & 80.0 & 6.40 & 219.6 & 37.5 & 55.0 & 154 & 12 & 35 & 00 & 249 & 20 & KS \\
\hline 29 & $01 / 20 / 64$ & -20.70 & 169.90 & 141.0 & 6.10 & 97.6 & 39.0 & -50.0 & 93 & 63 & 340 & 12 & 244 & 24 & IM \\
\hline 30 & $01 / 22 / 64$ & 22.40 & 93.60 & 88.0 & 6.10 & 238.0 & 18.9 & 0.0 & 220 & 4 & 7 & 42 & 208 & 19 & LTM \\
\hline 31 & $01 / 26 / 64$ & -16.3 & -71. & 116.0 & 6.10 & 203.0 & 37.6 & -16.4 & 181 & 43 & 64 & 26 & 313 & 36 & $\mathrm{IM}$ \\
\hline 32 & $01 / 28 / 64$ & 36.48 & 70.95 & 197.0 & 6.30 & 340.0 & 80.0 & 90.0 & 70 & 3 & 250 & & 160 & 0 & New \\
\hline 33 & $02 / 27 / 64$ & 21.70 & 94.40 & 102.0 & 6.40 & 263.3 & 19.6 & 30.8 & 220 & 33 & 76 & 52 & 321 & 17 & LTM \\
\hline 34 & $04 / 07 / 64$ & 0.10 & 123.2 & 184.0 & 6.30 & 169.2 & 60.3 & -179.2 & 30 & 21 & 128 & 20 & 258 & 60 & So73 \\
\hline 35 & $04 / 24 / 64$ & -5.10 & 144.20 & 106.0 & 6.30 & 356.3 & 10.0 & -87.3 & 83 & 55 & 264 & 35 & 172 & 1 & IM \\
\hline 36 & $05 / 26 / 64$ & -56.45 & -27.70 & 114.0 & $7.5 \mathrm{~B}$ & 179.3 & 52.7 & 162.0 & 41 & 14 & 156 & 30 & 297 & 49 & A72 \\
\hline 37 & $05 / 27 / 64$ & -56.40 & -28.4 & 61.0 & 6.00 & 281.0 & 48.2 & 44.7 & 221 & 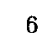 & 125 & 57 & 315 & 32 & IM \\
\hline 38 & $06 / 23 / 64$ & 43.30 & 146.10 & 77.0 & 6.20 & 200.5 & 13.4 & 38.9 & 152 & 36 & 351 & 52 & 249 & 9 & SMa \\
\hline 39 & $07 / 06 / 64$ & 18.30 & -100.40 & 45.0 & $7.3 \mathrm{~B}$ & 87.0 & 46.0 & -116.0 & 279 & 72 & 15 & 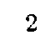 & 100 & 18 & MS \\
\hline 40 & $07 / 08 / 64$ & -5.50 & 129.80 & 165.0 & $7.0 \mathrm{~B}$ & 4.4 & 26.1 & 157.3 & 225 & 31 & 357 & 49 & 119 & 25 & FM \\
\hline
\end{tabular}




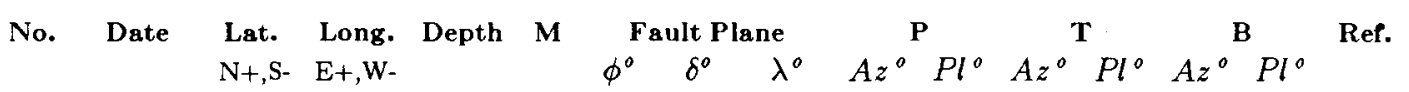

\begin{tabular}{|c|c|c|c|c|c|c|c|c|c|c|c|c|c|c|c|}
\hline 41 & $07 / 09 / 64$ & -15.50 & 167.60 & 127.0 & $7.4 \mathrm{~B}$ & 353.0 & 36.0 & 90.0 & 263 & 9 & 83 & 81 & 173 & 0 & IM \\
\hline 42 & $07 / 12 / 64$ & 24.90 & 95.30 & 155.0 & 6.70 & 258.0 & 61.2 & -9.0 & 219 & 26 & 122 & 14 & 6 & 60 & 071 \\
\hline 43 & $09 / 12 / 64$ & -4.40 & 144.00 & 120.0 & 6.30 & 305.0 & 2.0 & 90.0 & 215 & 43 & 35 & 47 & 125 & 0 & IM \\
\hline 44 & $09 / 15 / 64$ & 8.90 & 93.03 & 89.0 & 6.30 & 263.6 & 29.4 & 43.9 & 207 & 22 & 75 & 59 & 306 & 21 & LTM \\
\hline 45 & $11 / 01 / 64$ & 3.10 & 128.10 & 89.0 & 6.30 & 220.4 & 52.3 & 146.3 & 94 & 7 & 192 & 48 & 358 & 41 & F72 \\
\hline 46 & $11 / 02 / 64$ & -4.10 & -76.90 & 91.0 & 6.00 & 144.9 & 43.2 & -95.8 & 299 & 85 & 59 & 2 & 149 & 4 & IM \\
\hline 47 & $11 / 17 / 64$ & -5.70 & 150.70 & 65.0 & 6.70 & 266.0 & 32.5 & 90.0 & 176 & 13 & 356 & 78 & 86 & 0 & IM \\
\hline 48 & $11 / 24 / 64$ & -6.80 & 107.40 & 130.0 & 6.00 & 230.0 & 70.0 & 0.0 & 187 & 14 & 93 & 14 & 320 & 70 & So73 \\
\hline 49 & $01 / 02 / 65$ & 19.10 & 145.40 & 136.0 & 6.50 & 235.2 & 28.7 & -133.7 & 43 & 60 & 177 & 22 & 275 & 19 & KS \\
\hline 50 & $01 / 16 / 65$ & -56.60 & -27.40 & 101.0 & 6.10 & 158.0 & 15.0 & 90.0 & 68 & 30 & 248 & 60 & 338 & 0 & IM \\
\hline 51 & $02 / 23 / 65$ & -25.70 & -70.50 & 80.0 & 7.25 & 138.8 & .8 & 44.6 & 291 & 51 & 93 & 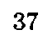 & 193 & 10 & S73 \\
\hline 52 & $03 / 04 / 65$ & -5.40 & 147.00 & 191.0 & 6.40 & 284.6 & 56.7 & -32.6 & 252 & 45 & 158 & $x^{2}$ & 63 & 45 & 071 \\
\hline 53 & $03 / 14 / 65$ & 36.42 & 70.73 & 205.0 & $7.5 \mathrm{~B}$ & 270.0 & 22.0 & 84.0 & 184 & 23 & 10 & 67 & 276 & 2 & O71 \\
\hline 54 & $03 / 22 / 65$ & -15.30 & -173.40 & 44.0 & 6.50 & 10.0 & 31.8 & -174.0 & 339 & 34 & 212 & 40 & 93 & 30 & ISO \\
\hline 55 & $03 / 28 / 65$ & -32.40 & -71.20 & 72.0 & 7.30 & 348.0 & 80.0 & -100.0 & 246 & 54 & 86 & 4 & 350 & 10 & MDM \\
\hline 56 & $03 / 31 / 65$ & 38.60 & 22.40 & 78.0 & 6.75 & 348.4 & 35.0 & 127.2 & 235 & 17 & 2 & & 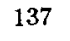 & 18 & IM \\
\hline 57 & $04 / 29 / 65$ & 47.40 & -122.40 & 63.0 & 6.88 & 344.0 & 70.0 & -75.0 & 277 & 62 & 62 & 24 & 159 & 14 & LB \\
\hline 58 & $05 / 17 / 65$ & 22.41 & 121.26 & 72.0 & 6.00 & 196.5 & 56.5 & 4.7 & 155 & 20 & 55 & 26 & 278 & 56 & KS \\
\hline 59 & $05 / 26 / 65$ & -56.10 & -27.60 & 120.0 & 6.70 & 175.9 & 57.2 & 140.7 & 52 & 0 & 146 & 00 & 32 & 41 & IM \\
\hline 60 & $06 / 12 / 65$ & -20.50 & -69.30 & 102.0 & 6.50 & 95.0 & 18.2 & -145.0 & 260 & 53 & 49 & 33 & 14 & 15 & S73 \\
\hline 61 & $7 / 02 / 65$ & 53.10 & -167.60 & 60.0 & 6.90 & 121.5 & 9.4 & -34.3 & 138 & 50 & 339 & 39 & 24 & 8 & IM \\
\hline 62 & $07 / 30 / 65$ & -18.10 & -70.80 & 72.0 & 6.00 & 232.6 & 24.6 & -2.6 & 211 & 41 & 77 & 39 & 32 & 25 & S73 \\
\hline 63 & $08 / 05 / 65$ & -5.20 & 151.60 & 50.0 & 6.50 & 44.0 & 50.0 & 96.8 & 129 & 5 & 357 & 83 & 22 & 5 & JoM \\
\hline 64 & $08 / 20 / 65$ & -18.90 & -69.00 & 128.0 & 6.50 & 206.0 & 15.2 & -30.6 & 221 & 51 & 67 & 36 & 328 & 13 & S73 \\
\hline 65 & $08 / 20 / 65$ & -22.80 & -176.20 & 79.0 & 6.10 & 313.0 & 5.0 & 55.1 & 263 & 40 & 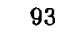 & I & 35 & 6 & ISO \\
\hline 66 & $09 / 16 / 65$ & 7.10 & 126.60 & 183.0 & 6.00 & 115.6 & 56.6 & 36.9 & 60 & 2 & 328 & 48 & 152 & 42 & $\mathrm{FM}$ \\
\hline 67 & $09 / 17 / 65$ & -1.50 & -77.70 & 191.0 & 6.50 & 82.6 & 13.4 & -143.0 & 242 & 52 & 3 & 36 & 135 & 11 & IM \\
\hline 68 & $09 / 21 / 65$ & 29.00 & 128.10 & 199.0 & 6.75 & 139.1 & 34.7 & -149.0 & 329 & 52 & 90 & 22 & 19 & 30 & KS \\
\hline 69 & $10 / 25 / 65$ & 44.20 & 145.30 & 181.0 & 6.50 & 178.0 & 16.8 & 26.1 & 139 & 36 & 346 & 50 & 237 & 14 & IM \\
\hline 70 & $11 / 12 / 65$ & 30.70 & 140.10 & 65.0 & 6.75 & 10.0 & 42.8 & -75.8 & 15 & 8 & 270 & $\Omega$ & 180 & 10 & KS \\
\hline 71 & $11 / 20 / 65$ & -7.30 & 129.20 & 132.0 & 6.10 & 233.4 & 29.4 & 136.1 & 110 & 22 & 241 & 59 & 10 & 22 & FM \\
\hline 72 & $11 / 21 / 65$ & -6.30 & 130.30 & 132.0 & 6.60 & 236.4 & 32.3 & 149.9 & 10 & 24 & 23 & 52 & 3 & 25 & FM \\
\hline 73 & $12 / 07 / 65$ & -6.40 & 146.30 & 116.0 & 6.10 & 339.0 & 67.8 & -2.2 & 297 & 17 & 202 & 14 & 77 & 68 & $R 74$ \\
\hline 74 & $12 / 08 / 65$ & -37.10 & 177.50 & 156.0 & 6.20 & 117.5 & 29.5 & 56.2 & 52 & 19 & 275 & 65 & 148 & 16 & CK80 \\
\hline 75 & $12 / 13 / 65$ & -56.10 & -27.60 & 153.0 & 6.30 & 215.0 & 55.0 & 138.7 & 94 & 2 & 180 & 51 & 2 & 38 & O71 \\
\hline 76 & $12 / 26 / 65$ & -5.40 & 151.60 & 76.0 & 6.10 & 331.0 & 24.6 & -180.0 & 174 & 40 & 308 & 40 & 60 & 25 & IM \\
\hline 77 & $02 / 03 / 66$ & 0.10 & 123.50 & 165.0 & 6.00 & 270.0 & 9.0 & 90.0 & 180 & 36 & 0 & 54 & 90 & 0 & FM \\
\hline 78 & $05 / 01 / 66$ & -8.40 & -74.30 & 154.0 & 6.75 & 248.2 & 26.7 & -33.9 & 251 & 54 & 117 & 27 & 15 & 22 & S75 \\
\hline 79 & $07 / 01 / 66$ & 24.80 & 122.40 & 119.0 & 6.75 & 304.0 & 27.0 & 90.0 & 214 & 18 & 34 & 72 & 124 & 0 & KS \\
\hline 80 & $08 / 10 / 66$ & -20.10 & -175.40 & 95.0 & 6.50 & 110.3 & 23.8 & -145.4 & 282 & 54 & 62 & 29 & 163 & 19 & ISO \\
\hline 81 & $08 / 21 / 66$ & 8.50 & 126.60 & 67.0 & 6.75 & 158.0 & 26.0 & 90.0 & 68 & 19 & 248 & 71 & 338 & 0 & F72 \\
\hline $8:$ & $09 / 08 / 66$ & 2.40 & 128.30 & 71.0 & $7.2 \mathrm{~B}$ & 326.7 & 41.3 & 98.1 & 231 & 4 & 2 & 83 & 140 & 4 & FM \\
\hline
\end{tabular}


TABLE IV (continued).

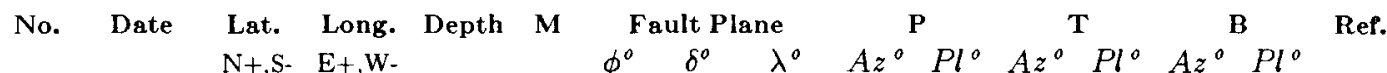

\begin{tabular}{|c|c|c|c|c|c|c|c|c|c|c|c|c|c|c|c|}
\hline$\because 3$ & $10 / 07 / 66$ & -21.60 & 170.60 & 160.0 & 6.30 & 9.5 & 69.3 & 157.6 & 59 & 0 & 328 & 30 & 149 & 60 & IM \\
\hline 4 & $11 / 10 / 66$ & -31.90 & -68.40 & 113.0 & 6.00 & 49.7 & 46.2 & -35.9 & 28 & 52 & 284 & 11 & 186 & 36 & S73 \\
\hline 5 & $12 / 01 / 66$ & -14.00 & 167.10 & 132.0 & 6.00 & 335.7 & 42.1 & 85.3 & 249 & 3 & 122 & 86 & 339 & 4 & IM \\
\hline 6 & $12 / 14 / 66$ & -4.89 & 144.00 & 70.0 & 6.00 & 122.0 & 30.0 & 90.0 & 32 & 15 & 212 & 75 & 302 & 0 & IM \\
\hline 87 & $01 / 19 / 67$ & -11.85 & 166.40 & 156.0 & 6.00 & 162.0 & 37.8 & 74.6 & 83 & 8 & 317 & 78 & 174 & 9 & IM \\
\hline $87^{\prime}$ & $02 / 17 / 67$ & -23.75 & -175.23 & 46.0 & 6.20 & 214.0 & 56.0 & 90.0 & 124 & 79 & 304 & 11 & 34 & 0 & JoM \\
\hline 88 & $02 / 19 / 67$ & -9.13 & 113.05 & 94.0 & 6.75 & 147.1 & 8.0 & -83.2 & 229 & 53 & 51 & 37 & 320 & 1 & F72 \\
\hline 39 & $03 / 11 / 67$ & -10.77 & 166.26 & 49.0 & 6.25 & 290.4 & 29.3 & -52.3 & 309 & 63 & 173 & 20 & 76 & 17 & JoM \\
\hline 90 & $04 / 12 / 67$ & 5.26 & 96.40 & 55.0 & 6.10 & 98.0 & 28.0 & 90.0 & 8 & 17 & 188 & 73 & 278 & 0 & F72 \\
\hline 1 & $05 / 11 / 67$ & -20.26 & -68.69 & 79.0 & 6.75 & 190.6 & 25.2 & -46.2 & 209 & 59 & 68 & 25 & 330 & 17 & S73 \\
\hline 92 & $05 / 21 / 67$ & 0.97 & 101.35 & 172.0 & 6.70 & 323.1 & 20.0 & 76.4 & 244 & 25 & 77 & 65 & 335 & 5 & FM \\
\hline 3 & $06 / 17 / 67$ & -58.30 & -26.60 & 140.0 & 6.10 & 147.0 & 22.6 & -114.4 & 272 & 64 & 73 & 24 & 170 & 8 & IM \\
\hline 94 & $07 / 29 / 67$ & 6.80 & -73.00 & 161.0 & 6.00 & 289.9 & 30.0 & 13.5 & 254 & 32 & 127 & 44 & 4 & 29 & MS \\
\hline 15 & $08 / 12 / 67$ & -24.70 & -177.50 & 134.0 & 6.50 & 37.5 & 26.7 & 43.7 & 342 & 24 & 204 & 58 & 81 & 19 & CK80 \\
\hline 96 & $08 / 26 / 67$ & 12.18 & 140.80 & 53.0 & 6.10 & 68.9 & 40.2 & -83.1 & 115 & 83 & 334 & 5 & 244 & 4 & KS \\
\hline 97 & $10 / 12 / 67$ & -7.10 & 129.80 & 86.0 & 6.20 & 84.8 & 56.1 & 37.7 & 29 & 2 & 296 & 49 & 122 & 41 & F72 \\
\hline 98 & $10 / 15 / 67$ & 11.90 & -86.00 & 162.0 & 6.20 & 304.2 & 18.1 & 67.5 & 232 & 28 & 68 & 61 & 326 & 7 & IM \\
\hline 99 & $10 / 25 / 67$ & 24.50 & 122.20 & 65.0 & $7.0 \mathrm{~B}$ & 33.3 & 42.7 & 133.4 & 274 & 10 & 18 & 60 & 180 & 28 & KSe \\
\hline 00 & $12 / 27 / 67$ & -21.20 & -68.30 & 135.0 & $7.0 \mathrm{~B}$ & 163.0 & 37.2 & -73.1 & 195 & 76 & 61 & 9 & 329 & 10 & S73 \\
\hline & $03 / 11 / 68$ & -16.20 & -173.90 & 112.0 & 6.00 & 2.8 & 47.7 & -30.6 & 339 & 48 & 234 & 12 & 137 & 39 & IM \\
\hline 02 & $05 / 28 / 68$ & -2.91 & 139.32 & 65.0 & $7.2 \mathrm{~B}$ & 118.9 & 39.0 & 94.1 & 26 & 6 & 180 & 84 & 296 & 3 & F72 \\
\hline 3 & $07 / 25 / 68$ & -30.77 & -178.35 & 60.0 & 6.40 & 192.0 & 30.0 & 90.0 & 102 & 15 & 282 & 75 & 12 & 0 & JoM \\
\hline & $08 / 05 / 68$ & 33.29 & 132.18 & 41.0 & 6.30 & 11.0 & 20.0 & -90.0 & 101 & 65 & 281 & 25 & 11 & 0 & New \\
\hline 05 & $09 / 09 / 68$ & -8.73 & -74.52 & 120.0 & 6.00 & 97.6 & 17.0 & -152.3 & 265 & 51 & 57 & 36 & 159 & 15 & S75 \\
\hline 6 & $09 / 20 / 68$ & 10.73 & -62.67 & 103.0 & 6.20 & 71.0 & 85.0 & 95.0 & 156 & 40 & 346 & 50 & 251 & 5 & S\&al \\
\hline 07 & $09 / 28 / 68$ & -13.16 & -76.38 & 70.0 & 6.00 & 122.0 & 16.6 & -125.5 & 257 & 57 & 59 & 32 & 156 & 10 & S75 \\
\hline 08 & $10 / 28 / 68$ & -12.47 & 166.46 & 60.0 & 6.50 & 282.7 & 47.2 & 36.3 & 228 & 10 & 125 & 52 & 325 & 36 & JoM \\
\hline 09 & $11 / 17 / 68$ & 9.55 & -72.65 & $172 . *$ & 6.63 & 240.0 & $60 . *$ & 90.0 & 330 & 15 & 150 & 75 & 60 & 0 & New \\
\hline 110 & $12 / 17 / 68$ & 60.20 & -152.80 & $90 . *$ & 6.50 & 346.0 & 65.0 & $70 . *$ & 91 & 18 & 222 & 64 & 355 & 18 & New \\
\hline 11 & $01 / 05 / 69$ & -7.98 & 158.91 & $71 . *$ & $7.1 \mathrm{~B}$ & 114.0 & 76.0 & $-80 . *$ & 37 & 58 & 196 & 30 & 292 & 10 & New \\
\hline 112 & $01 / 11 / 69$ & -28.41 & -176.96 & 68.0 & 6.63 & 206.0 & 13.8 & 90.0 & 116 & 31 & 296 & 55 & 26 & 0 & JoM \\
\hline 113 & $01 / 19 / 69$ & -14.89 & 167.19 & 107.0 & 7.30 & 175.0 & 40.6 & 175.0 & 31 & 30 & 145 & 35 & 272 & 40 & CK78 \\
\hline 14 & $01 / 30 / 69$ & 4.80 & 127.44 & 70.0 & $7.1 \mathrm{~B}$ & 1.0 & 22.3 & 116.7 & 251 & 25 & 48 & 63 & 156 & 4 & F72 \\
\hline 15 & $06 / 14 / 69$ & -7.91 & 158.98 & 66.0 & 6.60 & 318.9 & 67.8 & -5.0 & 278 & 19 & 182 & 12 & 57 & 61 & R75 \\
\hline 116 & $07 / 16 / 69$ & 52.20 & 158.98 & 69.0 & 6.50 & 214.6 & 30.2 & 138.2 & 90 & 22 & 221 & 58 & 351 & 22 & SMa \\
\hline 117 & $10 / 17 / 69$ & 23.05 & 94.70 & 124.0 & 6.00 & 90.0 & 15.0 & 90.0 & 0 & 30 & 180 & 60 & 270 & 0 & LTM \\
\hline 18 & $10 / 26 / 69$ & -16.17 & -173.95 & 127.0 & 6.70 & 46.4 & 45.8 & 64.7 & 334 & 2 & 239 & 72 & 65 & 18 & $\mathrm{~B} 80$ \\
\hline 19 & $12 / 25 / 69$ & 15.79 & -59.64 & 42.0 & 7.50 & 168.0 & 74.0 & -105.0 & 58 & 58 & 270 & 27 & 172 & 14 & S\&al \\
\hline 120 & $01 / 08 / 70$ & -34.74 & 178.57 & 179.0 & $7.0 \mathrm{~B}$ & 217.1 & 36.8 & 131.0 & 101 & 16 & 252 & 59 & 2 & 23 & $\mathrm{~B} 80$ \\
\hline 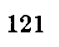 & $01 / 10 / 70$ & 6.82 & 126.74 & 73.0 & 7.30 & 179.1 & 45.7 & 77.3 & 98 & 0 & 6 & 81 & 190 & 9 & F72 \\
\hline 122 & $01 / 20 / 70$ & -25.80 & -177.35 & 82.0 & $7.2 \mathrm{~B}$ & 60.0 & 6.0 & 90.0 & 332 & 37 & 159 & 59 & 240 & 0 & B80 \\
\hline & $01 / 20 / 70$ & 2 & 142.97 & 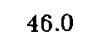 & 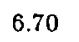 & 13.4 & 20.9 & 147.1 & 239 & 32 & 28 & 53 & 140 & 15 & SMa \\
\hline
\end{tabular}




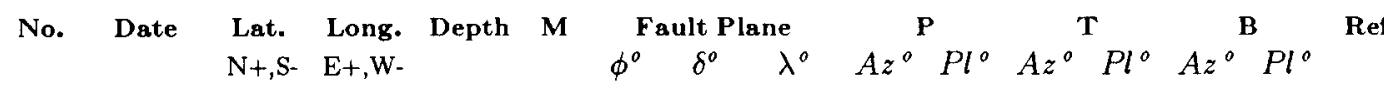

\begin{tabular}{|c|c|c|c|c|c|c|c|c|c|c|c|c|c|c|c|}
\hline 124 & $02 / 28 / 70$ & 2.70 & -175.10 & 159.0 & 6.10 & 171.0 & 27.0 & -116.3 & 313 & 67 & 102 & 20 & 195 & 12 & S72 \\
\hline 125 & $03 / 15 / 70$ & -29.65 & -69.50 & 119.0 & 6.00 & 332.2 & 25.3 & -102.8 & 90 & 70 & 252 & 20 & 344 & 5 & S73 \\
\hline 126 & $03 / 30 / 70$ & 6.80 & 126.65 & 76.0 & $7.1 \mathrm{~B}$ & 10.4 & 36.4 & 124.7 & 256 & 13 & 19 & 66 & 162 & 18 & F72 \\
\hline 127 & $05 / 20 / 70$ & -55.89 & -28.33 & 70.0 & 6.00 & 262.8 & 61.0 & 17.5 & 216 & 9 & 120 & 32 & 320 & 57 & Fo75 \\
\hline 128 & $05 / 31 / 70$ & -9.18 & -78.82 & 48.0 & 7.80 & 160.0 & 40.0 & -90.0 & 250 & 85 & 70 & 5 & 60 & 0 & S75 \\
\hline 129 & $06 / 11 / 70$ & -24.53 & -68.50 & 112.0 & 6.80 & 163.6 & 32.5 & -62.7 & 189 & 69 & 54 & 15 & 320 & 14 & S73 \\
\hline 130 & $06 / 17 / 70$ & -15.80 & -71.80 & 91.0 & 6.10 & 135.6 & 10.0 & -84.6 & 219 & 55 & 41 & 35 & 310 & 1 & S75 \\
\hline 131 & $06 / 19 / 70$ & -22.19 & -70.52 & 52.0 & 6.40 & 179.1 & 19.2 & -85.8 & 263 & 63 & 86 & 27 & 355 & 1 & $\$ 73$ \\
\hline 132 & $06 / 25 / 70$ & -7.92 & 158.69 & 69.0 & 6.50 & 263.0 & 75.0 & -90.0 & 173 & 60 & 353 & 30 & 263 & 0 & P79 \\
\hline 133 & $07 / 29 / 70$ & 26.02 & 95.40 & 68.0 & 6.50 & 264.4 & 57.5 & -10.3 & 227 & 29 & 128 & 16 & 13 & 56 & LTM \\
\hline 134 & $08 / 13 / 70$ & -8.88 & 117.98 & 99.0 & 6.00 & 208.1 & 26.2 & 66.7 & 135 & 20 & 340 & 66 & 229 & 10 & CI \\
\hline 135 & $08 / 28 / 70$ & -4.57 & 153.05 & 76.0 & 6.20 & 303.8 & 30.0 & 114.5 & 196 & 17 & 339 & 69 & 103 & 12 & $\mathrm{R} 75$ \\
\hline 136 & $09 / 29 / 70$ & -13.52 & 166.55 & 59.0 & 6.60 & 344.0 & 79.0 & 82.0 & 81 & 34 & 244 & 55 & 346 & 0 & New \\
\hline 137 & $12 / 08 / 70$ & -30.70 & -71.21 & 50.0 & 6.40 & 331.2 & 25.3 & 78.4 & 250 & 20 & 86 & 70 & 342 & 5 & S73 \\
\hline 138 & $12 / 28 / 70$ & -5.16 & 153.62 & 61.0 & 6.50 & 204.0 & 80.0 & 90.0 & 294 & 35 & 114 & 55 & 24 & 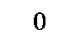 & P79 \\
\hline 139 & $12 / 29 / 70$ & -10.55 & 161.40 & 72.0 & 6.80 & 154.0 & 45.1 & 96.0 & 60 & 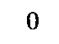 & 150 & 86 & 329 & 3 & R75 \\
\hline 140 & $02 / 21 / 71$ & -23.85 & -67.16 & 169.0 & 6.80 & 350.0 & 78.0 & -110.0 & 236 & 53 & 96 & 30 & 354 & 20 & New \\
\hline 141 & $03 / 13 / 71$ & -5.72 & 145.37 & 118.0 & 6.50 & 223.0 & 43.7 & 130.8 & 51 & 62 & 161 & 0 & 256 & 25 & R75 \\
\hline 142 & $03 / 23 / 71$ & -22.88 & -176.36 & 76.0 & 6.10 & 278.0 & 14.3 & 0.0 & 268 & 44 & 108 & 44 & 8 & 14 & B80 \\
\hline 143 & $04 / 07 / 71$ & 2.44 & 129.12 & 47.0 & 6.60 & 327.0 & 34.0 & -90.0 & 57 & 79 & 237 & 11 & 327 & 0 & CIK \\
\hline 144 & $04 / 08 / 71$ & -4.33 & 102.40 & 75.0 & 6.30 & 338.0 & 24.0 & 90.0 & 248 & 21 & 68 & 69 & 158 & 0 & New \\
\hline 145 & $05 / 08 / 71$ & -42.22 & -71.69 & $150 . *$ & 6.80 & $12 . *$ & $80 . *$ & $100 . *$ & 93 & 34 & 294 & 54 & 190 & 10 & New \\
\hline 146 & $05 / 17 / 71$ & -1.60 & -77.70 & 176.0 & 6.50 & 150.1 & 20.8 & -69.8 & 209 & 63 & 45 & 26 & 311 & 7 & S75 \\
\hline 147 & $06 / 11 / 71$ & 17.97 & -69.78 & 70.0 & 6.50 & 275.0 & 80.0 & 90.0 & 5 & 35 & 185 & 55 & 95 & 0 & New \\
\hline 148 & $06 / 17 / 71$ & -25.48 & -69.15 & 93.0 & $7.2 \mathrm{~B}$ & 2.0 & 70.0 & -104.0 & 250 & 62 & 103 & 24 & 7 & 13 & New \\
\hline 149 & $07 / 08 / 71$ & -7.03 & 129.70 & 92.0 & $7.3 \mathrm{~B}$ & 184.0 & 20.0 & 90.0 & 94 & 25 & 274 & 65 & 4 & 0 & New \\
\hline 150 & $07 / 19 / 71$ & $-5,69$ & 153.80 & 42.0 & 7.10 & 324.0 & 80.0 & -80.0 & 246 & 54 & 45 & 34 & 142 & 10 & New \\
\hline 151 & $07 / 27 / 71$ & -2.75 & -77.43 & 135.0 & 7.50 & 208.2 & 46.4 & -54.7 & 194 & 65 & 94 & 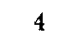 & 2 & 25 & S75 \\
\hline 152 & $08 / 02 / 71$ & 41.38 & 143.46 & 51.0 & 7.00 & 94.3 & 40.2 & -82.5 & 135 & 83 & 359 & 5 & 269 & 5 & SMa \\
\hline 153 & $09 / 16 / 71$ & -5.92 & 130.65 & 115.* & 6.20 & 220.0 & 78.0 & -90.0 & 130 & 57 & 310 & 33 & 220 & 0 & New \\
\hline 154 & $09 / 25 / 71$ & -6.54 & 146.57 & 115.0 & 6.30 & 320.1 & 34.4 & -47.1 & 329 & 61 & 200 & 17 & 103 & 20 & $R 75$ \\
\hline 155 & $09 / 28 / 71$ & -5.57 & 153.99 & 107.0 & 6.60 & 63.8 & 40.1 & 44.2 & 5 & 13 & 255 & 59 & 103 & 28 & R75 \\
\hline 156 & $11 / 21 / 71$ & -11.82 & 166.53 & $115 . *$ & $7.4 \mathrm{~B}$ & 118.0 & 45.0 & $116 . *$ & 10 & ${ }^{\circ}$ & 109 & 72 & 279 & 18 & New \\
\hline 157 & $11 / 24 / 71$ & 52.90 & 159.19 & 106.0 & $7.3 \mathrm{~B}$ & 30.0 & 86.0 & 90.0 & 120 & 41 & 300 & 49 & 210 & 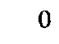 & New \\
\hline 158 & $12 / 30 / 71$ & -4.73 & 151.87 & 109.0 & 6.00 & 165.3 & 59.3 & -149.0 & 22 & 42 & 112 & 3 & 207 & 50 & R75 \\
\hline 159 & $01 / 08 / 72$ & -55.76 & -28.72 & $60 . *$ & 6.20 & 38.0 & 78.0 & $-70 . *$ & 332 & 53 & 112 & 30 & 214 & 20 & New \\
\hline 160 & $01 / 28 / 72$ & -19.38 & 169.11 & $124, *$ & 6.60 & 74.0 & 62.0 & $-80 . *$ & 6 & 71 & 157 & 16 & 249 & 9 & New \\
\hline 161 & $02 / 14 / 72$ & -11.36 & 166.33 & 102.0 & $7.0 \mathrm{~B}$ & 15.0 & 55.0 & 100.0 & 98 & 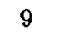 & 319 & 77 & 89 & 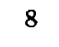 & New \\
\hline 162 & $02 / 26 / 72$ & -10.43 & 161.26 & 90.0 & 6.50 & 220.0 & 44.2 & 67.2 & 146 & 3 & 45 & 74 & 237 & 15 & R75 \\
\hline 163 & $03 / 07 / 72$ & -28.23 & -178.35 & 200.0 & 6.70 & 23.2 & 52.0 & -20.3 & 352 & 39 & 250 & 14 & 144 & 48 & B80 \\
\hline 164 & $03 / 19 / 72$ & 40.83 & 141.90 & 76.0 & 6.00 & 93.1 & 71.8 & -163.9 & 315 & 24 & 46 & 2 & 140 & 66 & $\mathrm{SMa}$ \\
\hline 165 & $03 / 22 / 72$ & 49.06 & 153.57 & 134.0 & 6.80 & 182.0 & 17.9 & -119.1 & 311 & 58 & 114 & 30 & 208 & 9 & $\mathrm{Mz}$ \\
\hline
\end{tabular}


Table IV (continued).

No. Date Lat. Long. Depth $M$ Fault Plane $P$ T $\quad$ B $\mathrm{N}+$, S- E+,W- $\quad \phi^{\circ} \quad \delta^{\circ} \quad \lambda^{\circ} \quad A z^{\circ} \quad P^{\circ} A z^{\circ} P^{\circ} A z^{\circ} P^{\circ}$

\begin{tabular}{|c|c|c|c|c|c|c|c|c|c|c|c|c|c|c|c|}
\hline 166 & $03 / 24 / 72$ & 56.14 & -157.18 & 71.0 & 6.00 & 326.6 & 38.6 & 110.6 & 222 & 8 & 342 & 75 & 130 & 13 & $\mathrm{HJ}$ \\
\hline 167 & $09 / 05 / 72$ & 1.88 & 128.17 & 132.0 & 6.10 & 153.0 & 42.0 & 90.0 & 63 & 3 & 243 & 87 & 333 & 0 & CIK \\
\hline 168 & $12 / 04 / 72$ & 33.33 & 140.68 & $62 . *$ & $7.4 \mathrm{~B}$ & 340.0 & 57.0 & 54.0 & 95 & 5 & 194 & 60 & 2 & 30 & New \\
\hline 169 & $01 / 05 / 73$ & -39.00 & 175.23 & 150.0 & 6.20 & 196.7 & 38.4 & 145.9 & 69 & 18 & 184 & 53 & 328 & 31 & D77 \\
\hline 170 & $01 / 18 / 73$ & -6.87 & 149.99 & 43.0 & 6.80 & 253.0 & 62.0 & 90.0 & 343 & 17 & 163 & 73 & 73 & 0 & P79 \\
\hline 171 & $01 / 27 / 73$ & 0.11 & 123.93 & 55.0 & 6.00 & 174.0 & 38.0 & 90.0 & 84 & 7 & 264 & 83 & 354 & 0 & CIK \\
\hline 172 & $03 / 09 / 73$ & 6.27 & 127.34 & 41.0 & 6.00 & 326.1 & 37.9 & -133.2 & 149 & 61 & 266 & 14 & 3 & 25 & CIK \\
\hline 173 & $04 / 03 / 73$ & 4.69 & -75.63 & 158.0 & 6.70 & 24.0 & 66.0 & -80.0 & 313 & 67 & 106 & 20 & 200 & g & New \\
\hline 174 & $04 / 24 / 73$ & 4.96 & -78.14 & 50.0 & 6.50 & 50.0 & 32.0 & -36.0 & 48 & 55 & 282 & 22 & 181 & 25 & New \\
\hline 175 & $06 / 09 / 73$ & -10.29 & 161.36 & $70 . *$ & 6.60 & 354.0 & 40.0 & 90.0 & 264 & 5 & 84 & 85 & 174 & 0 & New \\
\hline 176 & $06 / 17 / 73$ & 42.71 & 146.04 & 50.0 & 6.00 & 139.9 & 22.9 & -42.6 & 158 & 57 & 14 & 28 & 275 & 17 & SMa \\
\hline 177 & $08 / 01 / 73$ & -14.26 & 167.27 & 200.0 & 6.50 & 210.0 & 46.0 & 74.0 & 131 & 0 & 41 & 79 & 221 & 11 & New \\
\hline 178 & $08 / 28 / 73$ & 18.27 & -96.60 & 80.0 & $7.3 \mathrm{~B}$ & 326.0 & 50.0 & -75.0 & 297 & 78 & 45 & 4 & 136 & 11 & GMR \\
\hline 179 & $08 / 30 / 73$ & 7.34 & -72.83 & 181.0 & 6.50 & 182.0 & 82.0 & 90.0 & 272 & 37 & 92 & 53 & 2 & 0 & New \\
\hline 180 & $11 / 30 / 73$ & -15.20 & 167.39 & 124.0 & 6.00 & 16.5 & 38.4 & 93.5 & 284 & 7 & 86 & 83 & 194 & 2 & CK78 \\
\hline 181 & $12 / 29 / 73$ & -15.12 & 166.90 & 47.0 & 7.20 & 176.0 & 51.1 & 133.0 & 57 & 2 & 151 & 58 & 326 & 32 & CK78 \\
\hline 182 & $01 / 02 / 74$ & -22.54 & -68.40 & 105.0 & $7.1 \mathrm{~B}$ & 146.0 & 14.0 & -90.0 & 236 & 59 & 56 & 31 & 146 & 0 & New \\
\hline 183 & $01 / 05 / 74$ & -12.30 & -76.35 & 98.0 & 6.60 & 18.0 & 55.0 & -65.0 & 342 & 68 & 90 & 7 & 183 & 15 & New \\
\hline 184 & $10 / 08 / 74$ & 17.30 & -62.00 & 47.0 & 7.50 & 250.0 & 58.0 & -90.0 & 160 & 77 & 340 & 13 & 250 & 0 & S\&al \\
\hline 185 & $10 / 09 / 74$ & 44.72 & 150.12 & $55 . *$ & 6.70 & 222.0 & 14.0 & 90.0 & 132 & 31 & 312 & 59 & 42 & 0 & New \\
\hline 186 & $10 / 29 / 74$ & -6.88 & 129.46 & 156.0 & 6.60 & 304.0 & 51.0 & 164.0 & 169 & 17 & 272 & 37 & 58 & 48 & New \\
\hline 187 & $11 / 20 / 74$ & -15.12 & 167.16 & 62.0 & 6.20 & 168.0 & 37.3 & 92.0 & 77 & 15 & 248 & 82 & 346 & 1 & ISO \\
\hline 188 & $03 / 18 / 75$ & -4.23 & -77.02 & $75 . *$ & 6.50 & 8.0 & 65.0 & $-85 . *$ & 288 & 70 & 94 & 20 & 186 & 5 & New \\
\hline 189 & $04 / 09 / 75$ & -4.04 & 152.69 & 133.0 & 6.30 & 272.0 & 80.0 & 42.0 & 36 & 20 & 141 & 36 & 283 & 47 & New \\
\hline 190 & $05 / 27 / 75$ & 0.77 & 122.62 & 74.0 & 6.10 & 322.0 & 16.2 & -90.0 & 52 & 59 & 232 & 31 & 322 & 0 & CIK \\
\hline 191 & $07 / 08 / 75$ & 21.48 & 94.70 & 157.0 & 6.50 & 228.0 & 44.0 & 29.0 & 178 & 16 & 70 & 48 & 280 & 37 & LTM \\
\hline 192 & $07 / 10 / 75$ & 6.51 & 126.64 & 86.0 & 7.00 & 9.0 & 41.0 & 117.3 & 260 & 7 & 14 & 71 & 168 & 18 & CIK \\
\hline 193 & $08 / 10 / 75$ & -22.65 & -66.59 & 166.0 & 6.20 & 338.0 & 74.0 & -88.0 & 251 & 61 & 66 & 29 & 157 & 2 & New \\
\hline 194 & $08 / 23 / 75$ & 10.01 & 125.79 & 66.0 & 6.00 & 154.4 & 35.4 & 59.3 & 86 & 13 & 318 & 68 & 180 & 17 & CIK \\
\hline 195 & $10 / 17 / 75$ & -7.47 & 128.73 & $120 . *$ & 6.30 & 53.0 & 62.0 & $108 . *$ & 130 & 15 & 358 & 68 & 224 & 16 & New \\
\hline 196 & $11 / 01 / 75$ & 13.84 & 144.75 & $113 . *$ & 7.10 & 132.0 & 84.0 & 90.0 & 222 & 39 & 42 & 51 & 312 & 0 & New \\
\hline 197 & $12 / 25 / 75$ & -4.08 & 142.04 & $115 . *$ & 6.80 & 75.0 & 75.0 & $105 . *$ & 153 & 28 & 5 & 57 & 251 & 14 & New \\
\hline 198 & $01 / 24 / 76$ & -28.64 & -177.59 & 78.0 & 6.20 & 115.7 & 51.4 & 34.0 & 61 & 8 & 327 & 48 & 158 & 40 & R79 \\
\hline 199 & $03 / 04 / 76$ & -14.74 & 167.10 & 90.0 & 6.40 & 273.0 & 77.0 & 34.0 & 40 & 13 & 139 & 33 & 291 & 54 & New \\
\hline 200 & $06 / 03 / 76$ & -5.20 & 153.44 & $72 . *$ & 6.20 & 56.0 & 50.0 & $50 * *$ & 353 & 2 & 259 & 60 & 84 & 29 & New \\
\hline 201 & $11 / 07 / 76$ & 8.48 & 126.38 & 60.0 & 6.90 & 180.0 & 24.4 & 90.0 & 90 & 21 & 270 & 69 & 0 & 0 & CIK \\
\hline 202 & $11 / 30 / 76$ & -20.52 & -68.92 & 82.0 & $7.4 \mathrm{~B}$ & 342.0 & 78.0 & -108.0 & 230 & 54 & 87 & 31 & 346 & 18 & New \\
\hline 203 & $01 / 01 / 77$ & -7.91 & 109.01 & 102.8 & 6.00 & 63.0 & 23.0 & -130.0 & 217 & 60 & 3 & 26 & 101 & 15 & G84 \\
\hline 204 & $01 / 12 / 77$ & 1.58 & 99.88 & 186.0 & 6.00 & 92.0 & 46.0 & 132.0 & 334 & 6 & 75 & 60 & 240 & 29 & G84 \\
\hline 205 & $03 / 04 / 77$ & 45.77 & 26.76 & 94.0 & 7.40 & 255.0 & 76.0 & 90.0 & 345 & 31 & 165 & 59 & 75 & 0 & New \\
\hline 206 & $03 / 28 / 77$ & -14.68 & 167.10 & 111.9 & 6.07 & 165.0 & 53.0 & 164.0 & 30 & 16 & 132 & 36 & 280 & 50 & G84 \\
\hline 207 & $04 / 02 / 77$ & -16.70 & -172.10 & 50.3 & 7.28 & 317.0 & 41.0 & 45.0 & 258 & 12 & 147 & 60 & 354 & 28 & GDW \\
\hline
\end{tabular}




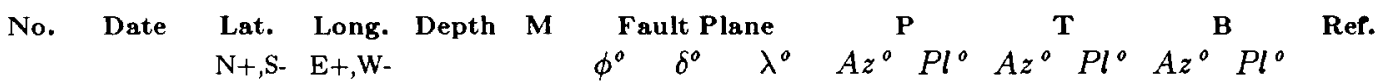

\begin{tabular}{|c|c|c|c|c|c|c|c|c|c|c|c|c|c|c|c|}
\hline 208 & $05 / 21 / 77$ & 15.70 & 20.82 & 188.0 & 6.12 & 329.0 & 48.0 & -15.0 & 298 & 38 & 192 & 20 & 81 & 46 & G84 \\
\hline 209 & $06 / 22 / 77$ & -22.88 & -175.90 & 59.1 & 8.04 & 10.0 & 13.0 & -98.0 & 111 & 58 & 287 & 32 & 18 & 2 & G84 \\
\hline 210 & $07 / 10 / 77$ & -56.12 & -27.56 & 116.7 & 6.03 & 166.0 & 56.0 & 140.0 & 43 & 1 & 134 & 51 & 312 & 39 & G84 \\
\hline 211 & $08 / 04 / 77$ & -56.01 & -27.79 & 111.1 & 5.94 & 171.0 & 47.0 & 143.0 & 46 & 10 & 149 & 53 & 309 & 36 & G84 \\
\hline $211^{\prime}$ & $08 / 11 / 77$ & -17.56 & -174.37 & 119.0 & 6.30 & 182.0 & 82.0 & -92.0 & 270 & 53 & 90 & 37 & 2 & 88 & K85 \\
\hline 212 & $10 / 29 / 77$ & -6.22 & 146.64 & 110.5 & 6.06 & 252.0 & 62.0 & -162.0 & 110 & 32 & 205 & 8 & 307 & 57 & G84 \\
\hline 213 & $10 / 30 / 77$ & -14.89 & 166.95 & 106.8 & 6.15 & 192.0 & 68.0 & 166.0 & 58 & 6 & 151 & 25 & 316 & 64 & G84 \\
\hline 214 & $12 / 31 / 77$ & -15.30 & -71.68 & 161.4 & 6.15 & 224.0 & 28.0 & -7.0 & 204 & 42 & 74 & 36 & 322 & 28 & G84 \\
\hline 215 & $01 / 28 / 78$ & -25.92 & -177.30 & 97.6 & 6.38 & 110.0 & 28.0 & 153.0 & 334 & 28 & 106 & 51 & 230 & 25 & G84 \\
\hline 216 & $04 / 18 / 78$ & 5.14 & 127.41 & 114.0 & 6.30 & 346.0 & 29.0 & 78.0 & 265 & 16 & 105 & 73 & 357 & 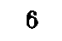 & G84 \\
\hline 217 & $05 / 01 / 78$ & -21.24 & 169.80 & 73.4 & 7.37 & 18.0 & 41.0 & -170.0 & 226 & 38 & 339 & 27 & 95 & 40 & G84 \\
\hline 218 & $05 / 13 / 78$ & -14.52 & 167.32 & 180.4 & 6.80 & 178.0 & 48.0 & 120.0 & 67 & 1 & 160 & 68 & 337 & 22 & G84 \\
\hline 219 & $05 / 23 / 78$ & 31.05 & 130.13 & 175.9 & 6.66 & 229.0 & 23.0 & 113.0 & 121 & 23 & 278 & 65 & 28 & 9 & G84 \\
\hline 220 & $08 / 03 / 78$ & -26.51 & -70.54 & 69.3 & 6.83 & 9.0 & 72.0 & -110.0 & 252 & 58 & 114 & 24 & 15 & 19 & New \\
\hline 221 & $09 / 02 / 78$ & 24.90 & 121.98 & 89.3 & 6.26 & 33.0 & 28.0 & 149.0 & 260 & 27 & 32 & 53 & 157 & 24 & G84 \\
\hline 222 & $09 / 06 / 78$ & -13.32 & 167.14 & 202.3 & 6.70 & 306.0 & 41.0 & 53.0 & 242 & 9 & 131 & 65 & 336 & 23 & G84 \\
\hline 223 & $12 / 06 / 78$ & 44.59 & 146.58 & 181.0 & 7.80 & 64.0 & 57.0 & -172.0 & 282 & 28 & 21 & 18 & 140 & 56 & G84 \\
\hline 224 & $02 / 07 / 79$ & 5.21 & 127.29 & 129.0 & 6.37 & 29.0 & 31.0 & 96.0 & 295 & 14 & 102 & 76 & 204 & 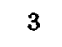 & G84 \\
\hline 225 & $02 / 11 / 79$ & 5.99 & 125.92 & 142.0 & 6.22 & 229.0 & 47.0 & 132.0 & 111 & 6 & 210 & 60 & 17 & 29 & G84 \\
\hline 226 & $02 / 14 / 79$ & -15.56 & 167.57 & 126.8 & 6.05 & 7.0 & 29.0 & 98.0 & 271 & 16 & 77 & 73 & 180 & 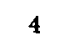 & G84 \\
\hline 227 & $05 / 20 / 79$ & 56.65 & -156.73 & 71.0 & 6.50 & 123.7 & 32.8 & 26.6 & 78 & 25 & 314 & 50 & 183 & 29 & $\mathrm{HJ}$ \\
\hline 228 & $06 / 22 / 79$ & 17.00 & -94.61 & 117.0 & 6.91 & 190.0 & 47.0 & -40.0 & 169 & 55 & 67 & 9 & 331 & 34 & G84 \\
\hline 229 & $06 / 25 / 79$ & -4.98 & 145.58 & 192.1 & 6.60 & 131.0 & 32.0 & -76.0 & 181 & 74 & 31 & 14 & 299 & . & G84 \\
\hline 230 & $08 / 05 / 79$ & -22.72 & -177.49 & 229.0 & 6.40 & 195.0 & 14.0 & -112.0 & 315 & 58 & 123 & 32 & 216 & 5 & G84 \\
\hline 231 & $08 / 22 / 79$ & 52.27 & 157.33 & 126.0 & 6.50 & 86.0 & 32.0 & 166.0 & 303 & 31 & & 77 & 192 & $\mathbf{3 1}$ & 384 \\
\hline 232 & $11 / 05 / 79$ & 17.83 & -68.62 & 78.3 & 6.19 & 93.0 & 22.0 & 87.0 & 5 & 23 & 188 & 67 & 96 & 1 & G84 \\
\hline 233 & $11 / 22 / 79$ & -24.34 & -67.39 & 181.0 & 6.22 & 209.0 & 44.0 & -37.0 & 191 & 54 & 84 & 12 & 345 & $8 \pi$ & G84 \\
\hline 234 & $11 / 23 / 79$ & 4.80 & -76.22 & 108.6 & 7.20 & 137.0 & 41.0 & -163.0 & 341 & 42 & 94 & 23 & 205 & 39 & G84 \\
\hline 235 & $12 / 11 / 79$ & 28.88 & 140.70 & 124.7 & 6.64 & 95.0 & 38.0 & 162.0 & 317 & 25 & 74 & 44 & 207 & 36 & G84 \\
\hline 236 & $02 / 29 / 80$ & 6.30 & 126.88 & 79.3 & 6.34 & 138.0 & 35.0 & 49.0 & 77 & 16 & 314 & 62 & 173 & 2 & G84 \\
\hline 237 & $04 / 13 / 80$ & -23.47 & -177.30 & 166.2 & 7.57 & 144.0 & 25.0 & -154.0 & 326 & 51 & 102 & 31 & 206 & 22 & G84 \\
\hline 238 & $05 / 14 / 80$ & -6.01 & 154.51 & 62.0 & 6.32 & 131.0 & 41.0 & 81.0 & 47 & - & 281 & 83 & 138 & 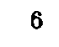 & GDW \\
\hline 239 & $05 / 26 / 80$ & -19.36 & -69.24 & 104.0 & 6.61 & 177.0 & 23.0 & -83.0 & 254 & 68 & 82 & 22 & 351 & 3 & G84 \\
\hline 240 & $06 / 16 / 80$ & -7.35 & 128.54 & 160.1 & 6.00 & 330.0 & 44.0 & 74.0 & 251 & o & 151 & 79 & 342 & 11 & G84 \\
\hline 241 & $06 / 18 / 80$ & -15.27 & -183.08 & 72.8 & 6.35 & 193.0 & 23.0 & 166.0 & 45 & 36 & 183 & 46 & 298 & 22 & SDW \\
\hline 242 & $06 / 25 / 80$ & 4.44 & -75.78 & 151.1 & 6.33 & 231.0 & 74.0 & 14.0 & 184 & 2 & 94 & 21 & 279 & 69 & GDW \\
\hline 243 & $07 / 08 / 80$ & 6.63 & 125.79 & 164.5 & 6.52 & 79.0 & 32.0 & 16.0 & 41 & 30 & 276 & 45 & 150 & 31 & G84 \\
\hline 244 & $07 / 16 / 80$ & -4.46 & 143.52 & 81.9 & 7.26 & 276.0 & 22.0 & 101.0 & 178 & 23 & 346 & 66 & 86 & 4 & GDW \\
\hline 245 & $07 / 19 / 80$ & -29.00 & -69.68 & 129.3 & 5.94 & 306.0 & 26.0 & -77.0 & 9 & 70 & 206 & 19 & 114 & 6 & G84 \\
\hline 246 & $07 / 22 / 80$ & -20.30 & 169.61 & 132.5 & 6.31 & 352.0 & 23.0 & -95.0 & 91 & 68 & 266 & 22 & 357 & 2 & G84 \\
\hline 247 & $10 / 08 / 80$ & -1.38 & -77.69 & 192.1 & 6.00 & 115.0 & 16.0 & -117.0 & 243 & 59 & 47 & 30 & 141 & 7 & G84 \\
\hline 248 & $10 / 24 / 80$ & 18.21 & -98.24 & 63.4 & 7.14 & 311.0 & 26.0 & -66.0 & 354 & 67 & 203 & 21 & 109 & 10 & GMR \\
\hline
\end{tabular}


Table IV (continued).

No. Date Lat. Long. Depth $M \quad$ Fault Plane $\quad P \quad$ T $\quad$ B $\mathrm{N}+$, S- E+,W- $\quad \phi^{\circ} \quad \delta^{\circ} \quad \lambda^{\circ} \quad \mathrm{Az}^{\circ} \quad \mathrm{Pl}^{\circ} \quad \mathrm{Az}^{\circ} \quad \mathrm{Pl}^{\circ} \mathrm{Az}^{\circ} \mathrm{Pl}^{\circ}$

\begin{tabular}{|c|c|c|c|c|c|c|c|c|c|c|c|c|c|c|c|}
\hline 249 & $01 / 23 / 81$ & 42.52 & 142.12 & 120.9 & 6.76 & 86.0 & 11.0 & -65.0 & 145 & 55 & 335 & 35 & 241 & 5 & DW \\
\hline 250 & $02 / 24 / 81$ & -6.06 & 148.74 & 77.8 & 6.56 & 288.0 & 37.0 & 108.0 & 185 & 9 & 315 & 76 & 93 & 11 & DW \\
\hline 251 & $03 / 26 / 81$ & -19.37 & -68.96 & 143.9 & 6.46 & 179.0 & 14.0 & -76.0 & 250 & 58 & 77 & 31 & 345 & 3 & DW \\
\hline 252 & $05 / 13 / 81$ & 5.83 & 127.01 & 133.1 & 6.32 & 317.0 & 39.0 & 111.0 & 212 & 8 & 332 & 75 & 120 & 13 & DW \\
\hline 253 & $05 / 22 / 81$ & -6.56 & 132.25 & 80.0 & 6.07 & 72.0 & 66.0 & 11.0 & 27 & 9 & 292 & 24 & 136 & 64 & DW \\
\hline 254 & $05 / 28 / 81$ & -14.69 & 167.28 & 138.0 & 6.16 & 185.0 & 60.0 & 150.0 & 57 & 3 & 150 & 41 & 324 & 49 & DW \\
\hline 255 & $06 / 26 / 81$ & -30.27 & -178.99 & 163.8 & 6.14 & 169.0 & 18.0 & -119.0 & 302 & 60 & 102 & 29 & 197 & 9 & DW \\
\hline 256 & $07 / 06 / 81$ & -22.26 & 171.73 & 58.3 & 7.54 & 345.0 & 30.0 & -179.0 & 191 & 38 & 318 & 37 & 74 & 30 & DW \\
\hline 257 & $08 / 07 / 81$ & -5.19 & 151.67 & 65.8 & 6.14 & 251.0 & 36.0 & 71.0 & 175 & 10 & 42 & 75 & 267 & 11 & DW \\
\hline 258 & $08 / 26 / 81$ & -5.34 & 151.48 & 63.6 & 5.94 & 259.0 & 29.0 & 77.0 & 179 & 17 & 20 & 72 & 270 & 6 & DW \\
\hline 259 & $09 / 03 / 81$ & 6.49 & 126.20 & 83.9 & 6.02 & 290.0 & 29.0 & -167.0 & 126 & 44 & 255 & 33 & 5 & 28 & DW \\
\hline 260 & $09 / 14 / 81$ & 18.32 & -68.89 & 159.6 & 6.02 & 78.0 & 25.0 & 85.0 & 352 & 20 & 178 & 70 & 83 & 2 & DW \\
\hline 261 & $10 / 17 / 81$ & -7.10 & 128.97 & 185.8 & 6.39 & 292.0 & 51.0 & 148.0 & 165 & 9 & 264 & 47 & 67 & 41 & DW \\
\hline 262 & $11 / 04 / 81$ & -20.02 & -174.39 & 56.6 & 6.51 & 19.0 & 8.0 & -71.0 & 87 & 52 & 272 & 37 & 180 & 3 & DW \\
\hline 263 & $11 / 07 / 81$ & -32.20 & -71.34 & 65.6 & 6.94 & 208.0 & 5.0 & -47.0 & 251 & 49 & 78 & 41 & 345 & 3 & DW \\
\hline 264 & $12 / 02 / 81$ & 40.91 & 142.51 & 83.3 & 6.28 & 224.0 & 69.0 & -162.0 & 85 & 27 & 176 & 3 & 272 & 63 & DW \\
\hline 265 & $12 / 11 / 81$ & -6.09 & 148.18 & 59.8 & 6.16 & 272.0 & 33.0 & 85.0 & 186 & 12 & 19 & 78 & 276 & 3 & DW \\
\hline 266 & $12 / 14 / 81$ & -56.36 & -26.48 & 71.1 & 6.04 & 234.0 & 37.0 & -155.0 & 70 & 48 & 188 & 23 & 294 & 33 & DW \\
\hline 267 & $01 / 04 / 82$ & -23.05 & -177.47 & 190.5 & 6.36 & 181.0 & 16.0 & -120.0 & 313 & 58 & 115 & 31 & 210 & 8 & $\mathrm{FiG}$ \\
\hline 268 & $02 / 27 / 82$ & 22.26 & 143.53 & 103.6 & 6.27 & 91.0 & 62.0 & 172.0 & 315 & 14 & 51 & 25 & 198 & 61 & $\mathrm{FiG}$ \\
\hline 269 & $03 / 10 / 82$ & -56.01 & -27.26 & 109.8 & 6.48 & 168.0 & 42.0 & 145.0 & 41 & 15 & 151 & 53 & 301 & 33 & $\mathrm{DFiG}$ \\
\hline 270 & $03 / 28 / 82$ & -12.77 & -76.09 & 108.7 & 6.31 & 32.0 & 17.0 & -13.0 & 27 & 46 & 240 & 39 & 136 & 17 & $\mathrm{DFiG}$ \\
\hline 271 & $03 / 29 / 82$ & 0.10 & 123.37 & 164.6 & 6.00 & 305.0 & 31.0 & 134.0 & 183 & 20 & 313 & 60 & 85 & 21 & $\mathrm{FiG}$ \\
\hline 272 & $04 / 16 / 82$ & -15.68 & -173.08 & 59.3 & 6.13 & 223.0 & 19.0 & 154.0 & 82 & 35 & 229 & 50 & 340 & 17 & $\mathrm{DFiG}$ \\
\hline 273 & $06 / 11 / 82$ & -17.53 & -174.46 & 113.2 & 6.80 & 237.0 & 16.0 & -38.0 & 259 & 53 & 105 & 34 & 6 & 13 & $\mathrm{DFiG}$ \\
\hline 274 & $06 / 19 / 82$ & 13.25 & -89.40 & 51.9 & 7.28 & 102.0 & 25.0 & -106.0 & 224 & 68 & 24 & 21 & 117 & 7 & DFiG \\
\hline 275 & $08 / 14 / 82$ & -5.06 & 143.91 & 114.1 & 6.03 & 30.0 & 42.0 & -15.0 & 4 & 40 & 252 & 24 & 140 & 40 & DFiG \\
\hline 276 & $08 / 26 / 82$ & -2.70 & -79.91 & 92.1 & 6.01 & 128.0 & 54.0 & 19.0 & 81 & 13 & 341 & 37 & 188 & 50 & $\mathrm{DFiG}$ \\
\hline 277 & $09 / 06 / 82$ & 29.31 & 140.28 & 155.6 & 6.80 & 100.0 & 55.0 & -158.0 & 313 & 39 & 52 & 11 & 155 & 49 & DFiG \\
\hline 278 & $09 / 15 / 82$ & -14.52 & -70.84 & 168.6 & 6.28 & 109.0 & 44.0 & -128.0 & 301 & 64 & 45 & 7 & 138 & 25 & $\mathrm{DFiG}$ \\
\hline 279 & $10 / 02 / 82$ & -14.74 & 167.28 & 154.6 & 6.02 & 190.0 & 51.0 & 147.0 & 63 & 8 & 163 & 48 & 326 & 41 & $\mathrm{DFiG}$ \\
\hline 280 & $10 / 31 / 82$ & 14.15 & -90.19 & 89.7 & 6.20 & 39.0 & 14.0 & -178.0 & 231 & 44 & 24 & 43 & 127 & 14 & $\mathrm{DFiG}$ \\
\hline 281 & $11 / 18 / 82$ & -1.73 & -76.74 & 190.2 & 6.54 & 163.0 & 29.0 & -51.0 & 180 & 62 & 45 & 21 & 308 & 18 & DFiG \\
\hline 282 & $11 / 18 / 82$ & -31.25 & -65.86 & 166.2 & 6.00 & 129.0 & 24.0 & -114.0 & 263 & 65 & 57 & 23 & 151 & 10 & DFiG \\
\hline 283 & $12 / 17 / 82$ & 24.64 & 122.62 & 94.4 & 6.47 & 54.0 & 49.0 & 152.0 & 284 & 12 & 27 & 46 & 183 & 42 & DFiG \\
\hline 284 & $01 / 01 / 83$ & -17.31 & -69.28 & 171.8 & 6.27 & 216.0 & 32.0 & -19.0 & 201 & 46 & 76 & 29 & 328 & 30 & DFiW \\
\hline 285 & $01 / 08 / 83$ & -15.32 & -173.40 & 52.9 & 6.25 & 214.0 & 11.0 & -150.0 & 15 & 50 & 176 & 39 & 274 & 10 & DFiW \\
\hline 286 & $01 / 24 / 83$ & 16.18 & -95.15 & 35.6 & 6.60 & 110.0 & 54.0 & -145.0 & 320 & 48 & 56 & 5 & 150 & 42 & DFiW \\
\hline 287 & $01 / 24 / 83$ & 12.95 & 93.64 & 72.7 & 6.76 & 110.0 & 54.0 & -145.0 & 320 & 48 & 56 & 5 & 150 & 42 & DFiW \\
\hline 288 & $01 / 26 / 83$ & -6.13 & 150.05 & 56.8 & 6.17 & 81.0 & 74.0 & 170.0 & 307 & 4 & 38 & 18 & 204 & 71 & DFiW \\
\hline 289 & $02 / 13 / 83$ & 13.84 & 144.98 & 108.1 & 6.10 & 154.0 & 36.0 & -23.0 & 138 & 47 & 18 & 24 & 272 & 33 & DFiW \\
\hline 290 & $02 / 25 / 83$ & -18.27 & -69.44 & 144.9 & 6.68 & 253.0 & 15.0 & -14.0 & 252 & 47 & 100 & 40 & 357 & 15 & DFiW \\
\hline
\end{tabular}




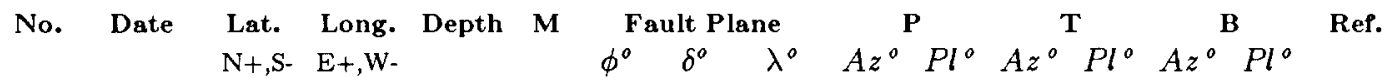

\begin{tabular}{|c|c|c|c|c|c|c|c|c|c|c|c|c|c|c|c|}
\hline 291 & $03 / 18 / 83$ & -4.86 & 153.51 & 69.9 & 7.71 & 170.0 & 49.0 & 120.0 & 59 & 0 & 150 & 68 & 329 & 22 & DFiW \\
\hline 292 & $03 / 20 / 83$ & -4.73 & 153.13 & 65.2 & 6.34 & 316.0 & 34.0 & 134.0 & 195 & 18 & 319 & 61 & 97 & 23 & DFiW \\
\hline 93 & $03 / 21 / 83$ & -21.67 & -175.32 & 51.5 & 6.65 & 357.0 & 16.0 & 84.0 & 272 & 29 & 96 & 01 & 3 & 2 & DFiW \\
\hline 294 & $04 / 04 / 83$ & 5.73 & 94.81 & 71.6 & 6.96 & 207.0 & 51.0 & 51.0 & 143 & 1 & 52 & 61 & 234 & 29 & DFWa \\
\hline 95 & $04 / 04 / 83$ & -15.02 & 167.27 & 119.8 & 6.08 & 180.0 & 34.0 & 146.0 & 51 & 21 & 173 & 54 & 309 & 28 & DFWa \\
\hline 296 & $04 / 12 / 83$ & -4.89 & -78.18 & 111.1 & 6.97 & 339.0 & 35.0 & -86.0 & 53 & 80 & 246 & 10 & 156 & 2 & $\mathrm{DFWa}_{\mathrm{a}}$ \\
\hline 297 & $04 / 18 / 83$ & 27.72 & 62.06 & 51.3 & 6.70 & 81.0 & 43.0 & -68.0 & 80 & 75 & 336 & 4 & 245 & 15 & $\mathrm{FWa}$ \\
\hline 298 & $05 / 10 / 83$ & -5.36 & 150.92 & 101.4 & 6.43 & 198.0 & 33.0 & -167.0 & 38 & 43 & 161 & 30 & 273 & 32 & PFWa \\
\hline 299 & $05 / 11 / 83$ & 2.30 & 128.35 & 133.1 & 6.09 & 198.0 & 27.0 & 153.0 & 62 & 29 & 195 & 51 & 318 & 24 & DFWa \\
\hline 300 & $06 / 01 / 83$ & -17.00 & -174.71 & 185.7 & 6.51 & 211.0 & 24.0 & -97.0 & 315 & 69 & 126 & 21 & 217 & 3 & $F W_{a}$ \\
\hline 301 & $07 / 24 / 83$ & 53.85 & 158.39 & 177.4 & 6.18 & 315.0 & 11.0 & 7.0 & 298 & 43 & 139 & 45 & 38 & 11 & $F W b$ \\
\hline 302 & $08 / 02 / 83$ & 20.46 & 122.17 & 166.2 & 6.24 & 0.0 & 23.0 & -79.0 & 70 & 67 & 262 & 22 & 170 & 4 & DFWb \\
\hline 303 & $08 / 06 / 83$ & -6.56 & 130.13 & 172.7 & 5.94 & 72.0 & 9.0 & -25.0 & 88 & 48 & 284 & 41 & 187 & 8 & DFWb \\
\hline 304 & $08 / 17 / 83$ & 55.79 & 161.21 & 77.2 & 7.01 & 216.0 & 41.0 & 60.0 & 147 & 8 & 36 & 69 & 240 & 19 & $F W b$ \\
\hline 305 & $08 / 25 / 83$ & 33.49 & 131.43 & 127.5 & 6.48 & 230.0 & 42.0 & 123.0 & 117 & 7 & 225 & 67 & 24 & 21 & $\mathrm{~Wb}$ \\
\hline 306 & $09 / 07 / 83$ & 60.98 & -147.50 & 52.1 & 6.33 & 26.0 & 41.0 & -97.0 & 169 & 84 & 301 & 4 & 31 & 5 & DFWb \\
\hline 307 & $09 / 14 / 83$ & 18.09 & 145.81 & 148.4 & 6.27 & 235.0 & 51.0 & -156.0 & 85 & 42 & 186 & 13 & 290 & 45 & DFWb \\
\hline 308 & $09 / 15 / 83$ & 16.11 & -93.23 & 121.5 & 6.29 & 138.0 & 9.0 & -107.0 & 248 & 54 & 63 & 36 & 155 & 3 & $\mathrm{~Wb}$ \\
\hline 309 & $10 / 27 / 83$ & 1.05 & 120.81 & 60.0 & 6.44 & 41.0 & 38.0 & 27.0 & 353 & 21 & 237 & 49 & 98 & 33 & DFWc \\
\hline 310 & $10 / 31 / 83$ & -9.11 & & 98.4 & 6.38 & 205.0 & 18.0 & 154.0 & 64 & 35 & 212 & -5 & 322 & 16 & DFWc \\
\hline 311 & $11 / 20 / 83$ & -7.53 & 130.67 & 78.5 & 6.09 & 73.0 & 60.0 & 11.0 & 29 & 14 & 29 & 2 & 14 & 58 & $\mathrm{DFW}_{\mathrm{c}}$ \\
\hline 312 & $11 / 24 / 83$ & -7.55 & 128.25 & 157.1 & 7.40 & 74.0 & 39.0 & 59.0 & 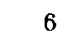 & 1 & 250 & & & 19 & Wc \\
\hline 313 & $12 / 10 / 83$ & 14.38 & -91.63 & 55.6 & 5.98 & 247.0 & 45.0 & 31.0 & 196 & 14 & 89 & 49 & 297 & 37 & $\mathrm{DFW}_{\mathrm{C}}$ \\
\hline 314 & $12 / 12 / 83$ & -7.70 & 127 . & 164.5 & 6.35 & 290.0 & 38.0 & 131.0 & 172 & 13 & 288 & 6 & 76 & 24 & DFWc \\
\hline 315 & $12 / 15 / 83$ & -33.09 & -70.28 & 103.6 & 6.00 & 202.0 & 21.0 & -78.0 & 271 & 65 & 103 & 2 & 11 & 4 & $\mathrm{DFW} \mathrm{c}$ \\
\hline 316 & $01 / 01 / 84$ & -3.86 & -78. & 125.9 & 5.68 & 327.0 & 35.0 & -46.0 & 328 & 61 & 206 & 17 & 109 & 23 & DFWd \\
\hline 317 & $01 /$ & -56.56 & & 113.6 & 5.64 & 219.0 & 40.0 & 151.0 & 88 & 19 & & 5 & 345 & 34 & DFWd \\
\hline 318 & $01 /$ & 36.39 & 71.03 & 172.0 & 6.03 & 68.0 & 34.0 & 89.0 & 339 & 11 & 162 & 79 & 69 & 1 & DFWd \\
\hline 319 & $02 / 16 / 84$ & 36.43 & 70.83 & 204.2 & 6.37 & 266.0 & 34.0 & 114.0 & 159 & 1 & 292 & $e^{-1}$ & 60 & 13 & DFWd \\
\hline 320 & $02 / 17 / 84$ & -6.60 & 130.12 & 172.1 & 5.96 & 216.0 & 26.0 & 103.0 & 116 & 19 & 279 & 70 & 24 & 6 & DFWd \\
\hline 21 & $02 / 26 / 84$ & -17.32 & -70.53 & 115.0 & 6.09 & 169.0 & 21.0 & -69.0 & 22 & & & & & 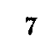 & DFWd \\
\hline 322 & $03 / 08 / 84$ & -38.25 & 177.22 & 78.5 & 6.01 & 310.0 & 49.0 & 8.0 & 184 & 10 & 85 & 50 & 86 & 39 & DFWd \\
\hline 323 & $03 / 23 / 84$ & -15.19 & -173. & 43.0 & 5.96 & 226.0 & 6.0 & -137.0 & 8 & 49 & 179 & 41 & 273 & 4 & DFWd \\
\hline 324 & $04 / 06 / 84$ & -18.90 & 168.85 & 175.0 & 6.80 & 304.0 & 14.0 & 42.0 & 254 & 35 & 95 & 53 & 351 & 10 & DFWe \\
\hline 325 & $04 / 13 / 84$ & -5.65 & 148.30 & 170.0 & 6.09 & 202.0 & 31.0 & -17.0 & 187 & 45 & 61 & 30 & 312 & 30 & DFWe \\
\hline 326 & $04 / 18 / 84$ & -15.93 & -174.35 & 158.0 & 6.44 & 198.0 & 21.0 & -76.0 & 264 & 65 & 97 & 24 & 5 & 5 & DFWe \\
\hline 327 & $05 / 29 / 84$ & 3.56 & 97.14 & 79.6 & 5.50 & 130.0 & 21.0 & -78.0 & 199 & 65 & 31 & 24 & 299 & 4 & DFWe \\
\hline 328 & $07 / 17 / 84$ & -56.39 & -27.39 & 118.0 & 6.00 & 348.0 & 35.0 & 29.0 & 300 & 22 & 179 & 51 & 44 & 30 & DFWf \\
\hline 329 & $09 / 30 / 84$ & -6.06 & & 49.9 & 5.93 & 258.0 & 32.0 & 82.0 & 174 & 13 & 1 & 76 & 265 & 4 & EDR \\
\hline 330 & $10 / 15 / 84$ & -15.86 & -173.64 & 119.7 & 7.07 & 161.0 & 89.0 & 105.0 & 56 & 44 & 265 & 42 & 161 & 15 & EDR \\
\hline 331 & & -24.07 & -00.83 & 192.0 & 6.15 & 220.0 & 27.0 & -67.0 & 26 & 00 & 113 & 20 & 19 & 10 & EDR \\
\hline 332 & $11 / 11 / 84$ & -12.69 & 166.74 & 88.0 & 5.66 & 213.0 & 67.0 & 96.0 & 298 & 22 & 134 & 67 & 31 & 6 & EDR \\
\hline
\end{tabular}


Table IV (continued).

\begin{tabular}{|c|c|c|c|c|c|c|c|c|c|c|c|c|c|c|c|}
\hline \multirow[t]{2}{*}{ No. } & \multirow[t]{2}{*}{ Date } & \multirow{2}{*}{$\begin{array}{l}\text { Lat. } \\
\mathrm{N}+, \mathrm{S}-\end{array}$} & \multirow{2}{*}{$\begin{array}{l}\text { Long. } \\
E+, W-\end{array}$} & \multirow[t]{2}{*}{ Depth } & \multirow[t]{2}{*}{$\mathbf{M}$} & \multicolumn{3}{|c|}{ Fault Plane } & \multicolumn{2}{|c|}{$\mathbf{P}$} & \multicolumn{2}{|c|}{$T$} & \multicolumn{2}{|c|}{ B } & \multirow[t]{2}{*}{ Ref. } \\
\hline & & & & & & $\phi^{0}$ & $\delta^{o}$ & $\lambda^{o}$ & $A z^{\circ}$ & $\mathrm{Pl}^{\circ}$ & $A z^{\circ}$ & $\mathrm{Pl}^{\circ}$ & $A z^{\circ}$ & $P l^{\circ}$ & \\
\hline 333 & $11 / 17 / 84$ & 47.52 & 154.49 & 42.0 & 5.70 & 202.0 & 84.0 & -22.0 & 156 & 20 & 250 & 11 & 7 & 67 & EDR \\
\hline 334 & $11 / 19 / 84$ & 51.78 & -175.28 & 47.0 & 5.65 & 253.0 & 31.0 & 107.0 & 151 & 15 & 299 & 73 & 58 & 9 & EDR \\
\hline 335 & $11 / 20 / 84$ & 5.17 & 125.12 & 180.7 & 7.48 & 335.0 & 66.0 & -97.0 & 232 & 68 & 70 & 21 & 338 & 6 & EDR \\
\hline
\end{tabular}

* parameter constrained by modeling of long-period $\mathrm{P}$ waves.

$M$ magnitudes are $m_{\mathrm{b}}$ for events before 1977, if a B appears next to the value, then it is $m_{\mathrm{B}}$ from Abe (1980); magnitudes are $M_{\mathrm{W}}$ from 1977 to 1984.

A72 - Abe, 1972; B80 - Billington, 1980; CI - Cardwell and Isacks, 1978; CIK - Cardwell, Isacks and Kraig, 1980; CK78 - Chung and Kanamori, 1978; CK80 - Chung and Kanamori, 1980; D77 - Denham, 1977; DW - Dziewonski and Woodhouse, 1983; DFiG Dziewonski, Friedman, Giardini and Woodhouse, 1983a; DFiW - Dziewonski, Friedman and Woodhouse, 1983b; DFWa Dziewonski, Franzen and Woodhouse, 1983c; DWWb - Dziewonski, Franzen and Woodhouse, 1984a; DFWc - Dziewonski, Franzen and Woodhouse, 1984b; DFWd - Dziewonski, Franzen and Woodhouse, 1984c; DFWe - Dziewonski, Franzen and Woodhouse, 1985a; DFWf - Dziewonski, Franzen and Woodhouse, 1985b; EDR - Earthquake Data Report, U.S.G.S., 1984; F72 - Fitch, 1972; FM - Fitch and Molnar, 1970; Fo75 - Forsyth, 1975; FK - Fujita and Kanamori, 1981; G84 - Giardini, (personal communication) 1984; GDW - Giardini, Dziewonski and Woodhouse, 1985; GMR - Gonzalez, McNally and Rial, 1984; HJ - House and Jacob, 1983; IM - Isacks and Molnar, 1969; ISO - Isacks, Sykes and Oliver, 1969; JoM - Johnson and Molnar, 1972; KS - Katsumata and Sykes, 1969; K85 - Kawakatsu, 1985; KSe - Kawakatsu and Seno, 1983; LB - Langston and Blum, 1977; LTM - LeDain, Tapponier and Molnar, 1984; MDM - Malgrange, Deschamps and Madariaga, 1981; MS - Molnar and Sykes, 1969; O71 - Oike, 1971; OA - Osada and Abe, 1981; P79 - Pascal, 1979; Ri79 - Richter, 1979; R74 - Ripper, 1974; R75 - Ripper, 1975; So73 - Soedarmo, 1973 ; S72 Stauder, 1972; S73 - Stauder, 1973; S75 - Stauder, 1975; SB - Stauder and Bollinger, 1966; SMa - Stauder and Maulchin, 1976; S \& al - Stein, Engeln, Wiens, Speed and Fujita, 1982.

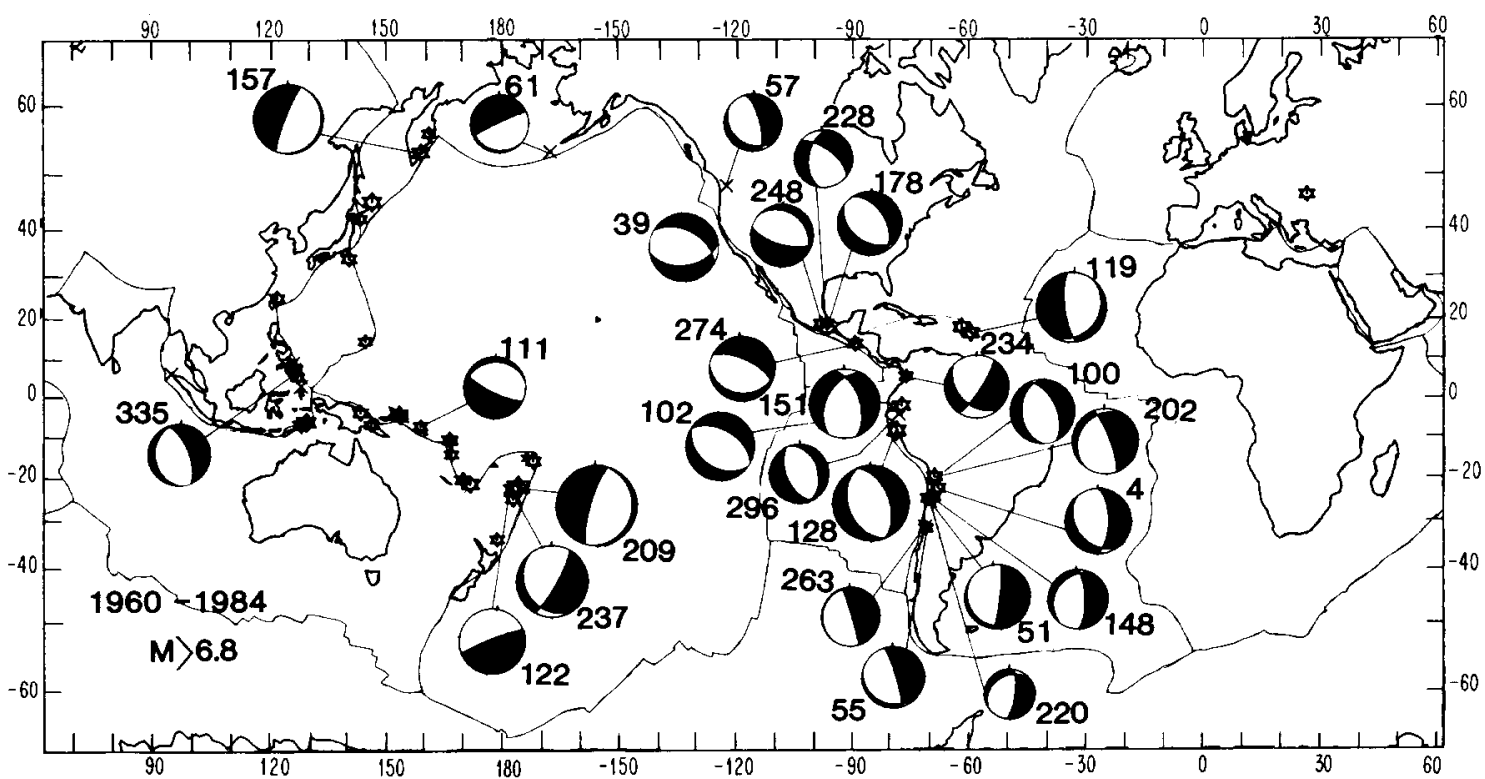

Fig. 5. Focal mechanisms of type 1 events that indicate normal faulting within the subducted plate and strike subparallel to the trench axis. The events shown are large $\left(M_{\mathrm{S}}>6.8\right)$ intermediate-depth earthquakes that occurred from 1960 to 1984 . The mechanism diagrams are lower hemisphere equal area projections that are proportional in size to the earthquake magnitudes. Dark quadrants are compressional. Numbers identify the events in Table IV. 


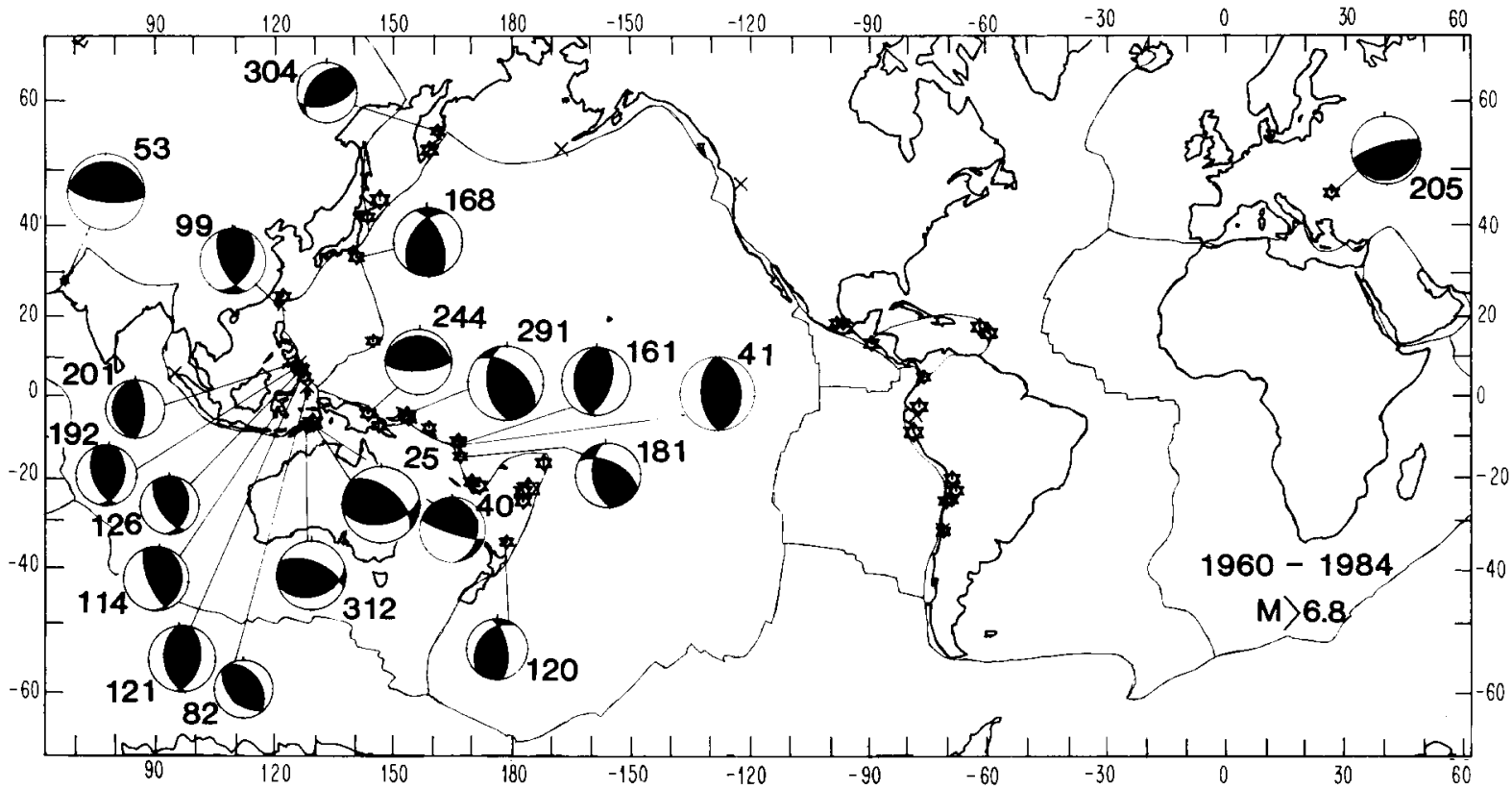

Fig. 6. Focal mechanisms of type 2 events that show reverse faulting within the subducted plate. The strike of these events is also subparallel to the trench axis. Numbers identify the events in Table IV. Dark quadrants are compressional. The diagrams are lower hemisphere equal area projections that have sizes proportional to the magnitude of the earthquakes.

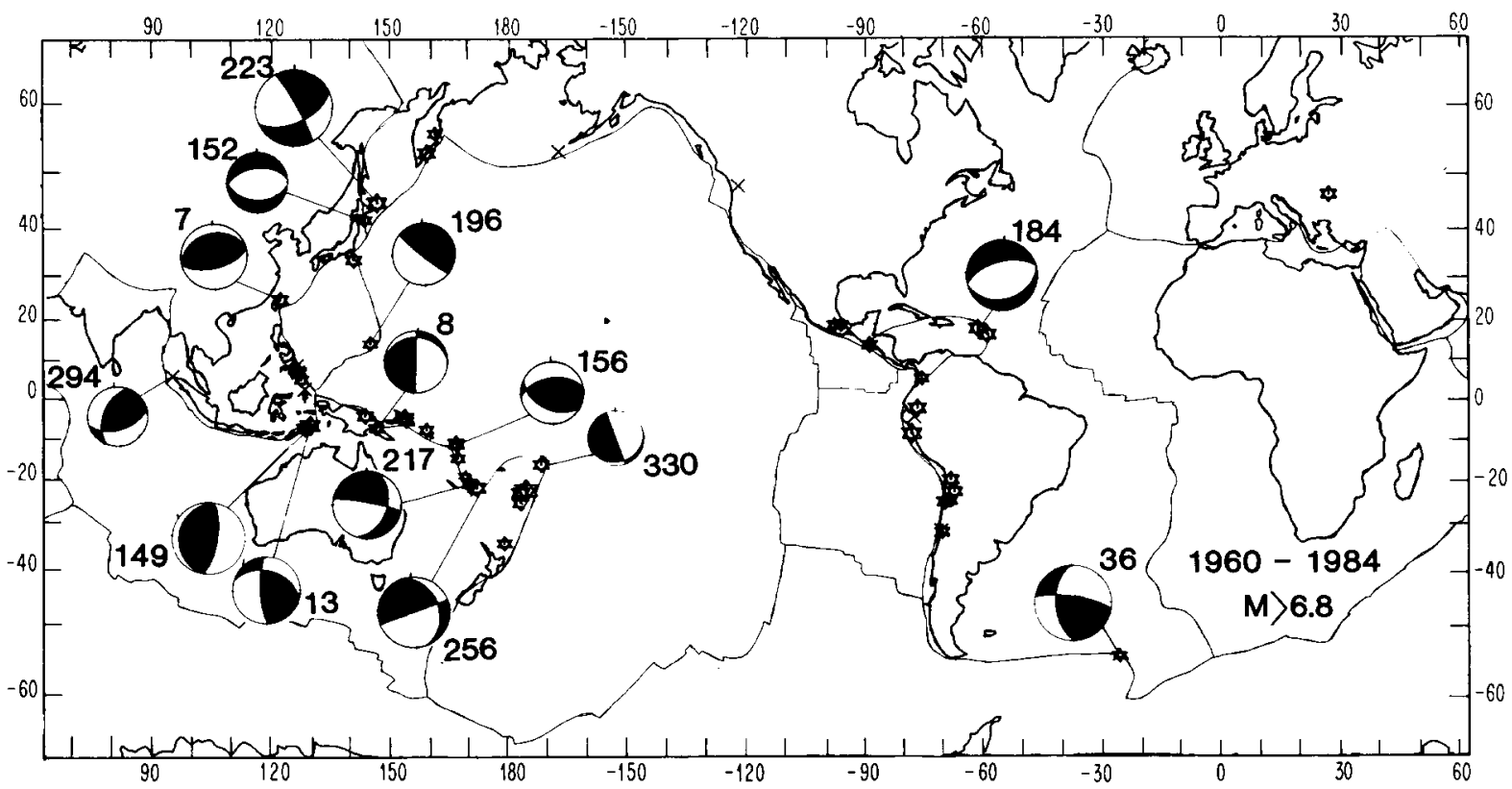

Fig. 7. Focal mechanisms of type 3 and 4 events are shown. These events indicate hinge faulting or contorsions within the subducted slab and tear faulting, respectively. Compressional quadrants are darkened. The mechanism diagrams are plotted in a lower hemisphere equal area projection. Their size is proportional to the earthquake's magnitude. Numbers identify the events in Table IV. 
fault planes are given as well as the pressure $(P)$, tension $(T)$ and intermediate $(B)$ stress axes. Azimuth is measured clockwise from north. Abbreviations are references that are listed at the bottom of Table IV.

This catalog probably includes most intermediate-depth intraplate earthquakes with $M \geq$ 6.5 that occurred within the subducting slab between 1960 and 1984. However, some intermediate-depth events that were originally listed by NOAA and ISC as shallow may be missing, particularly if they have compressional mechanisms. Also, a few of the events listed in the catalog may actually be interplate events with slightly anomalous depths and mechanisms. This is a significant expansion of the data base of 210 events in the same period compiled by Fujita and Kanamori (1981); 33 of their mechanisms are for events prior to 1960 , most of which are based on ISS reports, and only 75 of their other events are large enough to be included in Table IV.

If we consider only the largest of the events in this catalogue $(M>6.8)$, a regional pattern in the types of mechanisms emerges, as shown in Figs. 5-7. These events can be grouped as follows:

(1) normal-fault events (44\%) with a strike subparallel to the trench axis (Fig. 5);

(2) reverse-fault events (33\%) with a strike subparallel to the trench axis (Fig. 6);

(3) normal or reverse fault events with a strike significantly oblique to the trench axis $(10 \%$, Fig. 7);

(4) tear faulting events (13\%, Fig. 7).

Fault plane solutions of type 1 events (Fig. 5) indicate a steeply dipping normal fault, usually with the 'continental' side down-dropped. These events occur mainly along the South and Middle America trenches, but they also occur in the Pacific Northwest, Alaska, and Kamchatka. All of these zones are strongly or moderately coupled subduction zones (Kanamori, 1977b; Uyeda and Kanamori, 1979; Ruff and Kanamori, 1983) and the focal mechanisms of the intermediate-depth events are down-dip tensional. However, the type 1 events that occur along the Philippine and Solomon Island regions (335 and 111) are downdip compressional. The Solomon Islands region is the site of numerous large doublet events (Lay and
Kanamori, 1980; Wesnousky et al., 1986) that suggest moderate interplate coupling, although no single earthquake with $M>7.7$ has occurred there. Events 122, 209, and 237 in the Tonga trench may be related to subduction of the Louisville ridge, which may cause a localized increase in interplate coupling, in an otherwise uncoupled region. Note that event 209 is down-dip tensional and the largest event $\left(M_{\mathrm{W}}=8.0\right)$ in the catalog. This event may have fractured and detached the subducted slab at the leading edge of the coupled interplate boundary (Given and Kanamori, 1980). Events 122 and 237, on the other hand, are down-dip compressional and deeper than event 209 (Table IV). These events are discussed in more detail below. Simple models of plate coupling and geometry suggest that most type 1 events occur at strongly coupled plate boundaries, where a downdip extensional stress prevails in a gently dipping plate. Various explanations for the occurrence of these events include (1) bending or unbending stresses, (2) continental loading and (3) down-dip gravitational loading by the leading edge of the plate. The dominant mechanism may vary from region to region, as we will explore in a later section. One possible interpretation is that at strongly coupled subduction zones the negative buoyancy of the subducting plate tends to concentrate down-dip tensional stresses in the downgoing slab near the lower edge of the coupled thrust plane, as shown in Fig. 8a. Thus, the occurrence of type 1 events may be interpreted as evidence of strong coupling between plates. Although the coupling of the Pacific Northwest is being debated, note that the 1965 and 1949 Puget Sound earthquakes are type 1 events, which would suggest strong coupling between the Juan de Fuca and North American plates. This study may provide an additional clue to the strength of coupling in this region.

In contrast, if the interplate boundary is weakly coupled, the stress due to negative slab buoyancy is transferred to even shallower depth, causing large normal-fault events near the trench where the curvature of the plate is largest (Fig. 8b). The great 1933 Sanriku earthquake in northeast Japan (Kanamori, 1970; Mogi, 1973), and the 1977 Sumbawa, Java earthquake (Given and Kanamori, 


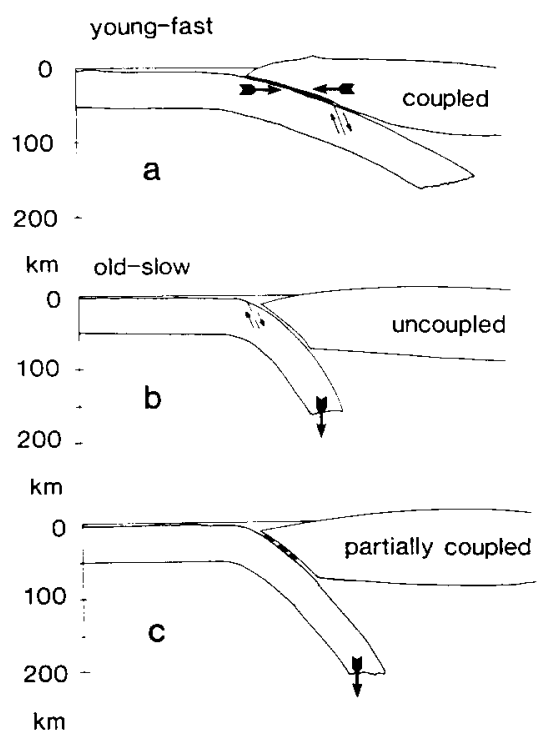

Fig. 8. A schematic figure showing (a) the possible mechanism of type 1 events at a strongly coupled plate boundary and (c) type 2 events at a moderately coupled boundary. Figure $8 b$ shows the case where the interplate boundary is decoupled, allowing slab pull stresses to produce large normal faulting events at shallow depths below the trench.

1980; Silver and Jordan, 1983; Spence, 1986), which occurred at weakly coupled subduction zones, are examples of this type.

Type 2 events have reverse-fault solutions and strike subparallel to the subduction zone (Fig. 6). The events that occur in the Philippines, northwest Solomon Islands, New Hebrides, and Kermadec regions show near vertical tension and horizontal compression axes. Note that all of these regions are considered partially coupled or uncoupled subduction zones (Kanamori, 1977b; Uyeda and Kanamori, 1979; Ruff and Kanamori, 1983), where the continuous seismicity is deeper than 300 $\mathrm{km}$ (see Table I). In terms of our simple model, the increased dip angle of the downgoing slab associated with weakly coupled subduction zones, together with the weight of the relatively long subducted plate, induces vertical tensional stresses at intermediate depth, which are responsible for the change in focal mechanism from type 1 to type 2 events (Fig. 8c). Exceptions are events 114 in New Guinea, 205 in Rumania, and 244 in the Philippines that show down-dip compressional stresses. We will discuss these three events in detail in the regional descriptions later.

Events of types 3 and 4 are shown in Fig. 7. Type 3 events $(7,149,152,156,184,294)$ occur where the trench axis bends sharply, causing horizontal extensional or compressional intraplate stresses. These events indicate hinge faulting within the subducting slab. Type 4 events $(8,13,36,196$, $217,223,256,330)$ include all those that do not fall in the above categories. These events occur at plate boundaries with complex features and may be related to tear faulting.

\section{Aftershock characteristics for intermediate- depth events}

Intermediate-depth earthquakes tend to have far fewer aftershocks than shallow subduction events with similar magnitudes. We determine the number of aftershocks of the events with $M \geq 6.5$ in Table V, reported by the NOAA and ISC catalogs, within one week after the main event. About $48 \%$ of the events had no aftershocks and $37 \%$ of the events had between 1 and 5 aftershocks. If we consider a one-month period, the number of aftershocks on various characteristics of the subducting slabs. Little correlation with slab age, maximum depth of seismicity or convergence rate was found.

Figure 9 shows the variation of the number of one-week aftershocks, $N$, with the mainshock magnitude for events with $M>6.5$ in Table IV. Note that most events have no aftershocks or only a few detected. As expected, there is a slight positive correlation between mainshock magnitude and number of aftershocks occurring within one week that could be attributed to detection threshold. However, there are some events with particularly large numbers of aftershocks shown on the map in Fig. 9. Open circles indicate events with $N>5$, filled circles $N>10$ and stars $N>25$ aftershocks. These events are generally large intermediate-depth events that are associated with bends in the subducted slab, or with moderately coupled or uncoupled regions like the December 25, 1969 (event 119, Stein et al., 1982) and the 

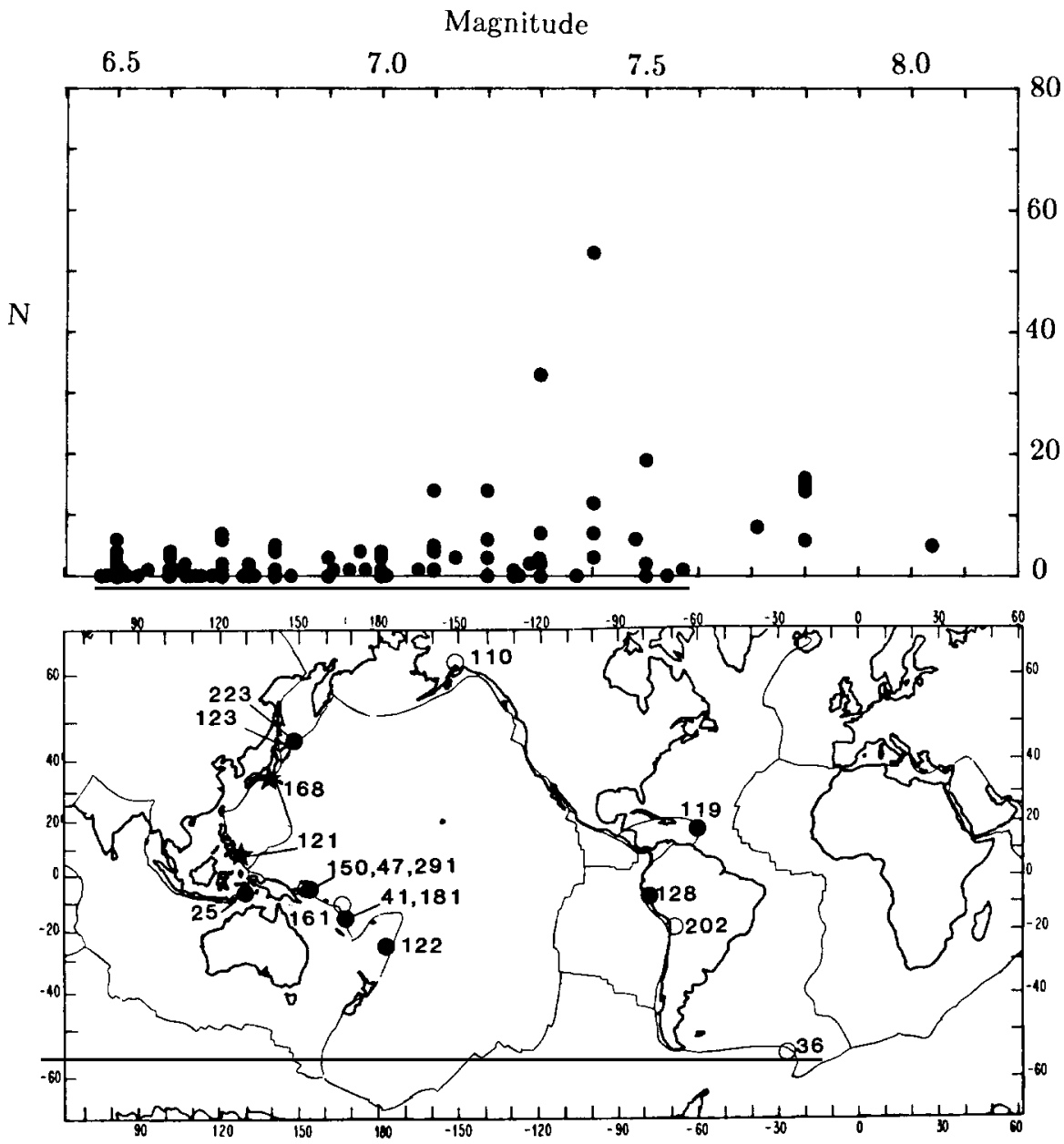

Fig. 9. The distribution of number of aftershocks with $M>3.5$ that occurred within one week of an intermediate-depth event and the magnitude of the mainshock is shown on the top diagram. Mainshocks are from 1960 to 1984. Note that most events have no aftershocks or only a few. Location of events (Table IV) with larger number of aftershocks are indicated in the map. Open circles indicate $N>5$, filled circles $N>10$, and stars $N>25$ aftershocks.

1972 Izu-Bonin event (168), which had the largest number of aftershocks within a week.

\section{Stress-axis distribution of intermediate-depth earthquakes}

In principle, we can determine the general intraplate stress field orientation for a region by plotting the $P$ and $T$ axes of focal mechanisms of events in that region in a focal sphere together with the downgoing plate orientation at the corre- sponding depth. This approach has been used by many investigators (e.g., Oike, 1971; Fujita and Kanamori, 1981; Vassiliou et al., 1984; Burbach and Frohlich, 1986). Since our data base has been expanded from previous studies we use this same method not only to determine the general characteristics of the stress field in each region but also to help us discriminate 'anomalous events'.

Figure 10 shows equal area lower hemisphere projections of the distribution of compression ( $P$ $=$ filled symbols) and tension ( $T=$ open symbols) axes for different subduction zones. Circles are 
used for events located between 40 and $100 \mathrm{~km}$ depth and diamonds for events $100-200 \mathrm{~km}$ deep. Region names are defined in Fig. 4 and open arrows indicate the convergence direction of the plates in each region (Table I). The curve indicates the trench azimuth and dip. The dip angle shown in Fig. 10 is that of the seismic zone at $-100 \mathrm{~km}$ depth. It is common to observe an abrupt increase of the seismicity dip angle at $40 \mathrm{~km}$ depth and the dip of the seismic zone increases gently to $\sim 100$ $\mathrm{km}$, remaining approximately constant thereafter (Spence, 1987). We define in-plate tension or compressional events on the basis of whether the respective stress axes are located within $20^{\circ}$ of the down-dip slab location. This method ignores earthquake magnitudes; but we believe that the larger events used here reflect the first-order stress distribution in a region, whereas smaller events reflect only secondary or local effects. Inspection of Fig. 10 indicates that most regions have either dominant down-dip tensional stresses or a mixed pattern of down-dip tensional and compressional stresses at intermediate depth. Only Tonga has dominant down-dip compressional stresses.

Most intermediate-depth events that occur in South America regions (from Colombia to Central Chile) have near in-plate tensional axes. Note also that the tension axis of all events in the Altiplano and North Chile regions are especially well aligned with the subducted plate and the convergence direction. These results agree with those of previous investigators (e.g., Stauder, 1973, 1975). The South Chile region has only one down-dip compression event that occurred on May 8, 1971. This event has been discussed in detail by Astiz and Kanamori (1986) who infer that the great underthrusting event in 1960 temporally induced intraplate compression down-dip. A large number of events in the Scotia region have nearly down-dip tensional axes. This result is consistent with the model of weak coupling at the interplate boundary in which near vertical tensional stress is induced as a result of the negative buoyancy of the slab.

The Mexico and Central America regions have mostly shallow-dipping tensional axes, consistent with the observed seismicity dip angle. In the Caribbean region only a few intermediate-depth events have occurred between 1960 and 1984. The
Greater Antilles events have nearly vertical tension axes consistent with the weakly coupled model described earlier. In contrast, the tension and compression axes of events in the Lesser Antilles are aligned with the trench axes. These events are discussed in more detail in the following section.

The 1965 Puget Sound event, that occurred in the Juan de Fuca region, has a down-dip tensional stress axis. The dip angle in this region is shallow $\left(22^{\circ}\right)$, consistent with a strongly coupled interplate boundary. The North Pacific regions are also considered strongly coupled regions and had only a few events at intermediate depth. Alaska has mostly down-dip tensional events, but events that occur in the Aleutians and Kamchatka regions have their stress axes widely distributed in the focal sphere.

The Kurile subduction zone is divided into two sections according to differences in the dip and depth extent of seismicity (see Table I). The stress axis distribution is mixed in both Kurile and the Northeast Japan regions. These two regions have been very active. However, only a few events have occurred within the oldest ( $>150 \mathrm{Ma}$ ) subducting oceanic lithosphere in the Izu-Bonin and Mariana regions. The deeper events in the Izu-Bonin region have in-plate compression axes; however, the events with depth $<100 \mathrm{~km}$ are mixed. The Mariana region was divided into northern and southern segments because of the abrupt change in the trench axis.

Events in the Ryukyu region have a mixed stress axis pattern, but it is aligned with the direction of convergence of the Eurasia and Philippine plates. The North Taiwan region events have mostly down-dip tensional axes which are nearly vertical (Seno and Kurita, 1978). Thus, this region is consistent with a rather weakly coupled interplate boundary. In the subducting plate in Luzon only three events have occurred, but the stress axes are distributed randomly which may reflect the complexity of this area. The Philippine subduction zone is among the more active seismic regions at intermediate depth (see Appendix 1). Most events in the northern section show nearly vertical tension axes that align with the steeply dipping seismic zone. In the southern segment, however, the tension axes of the events are mostly 
106
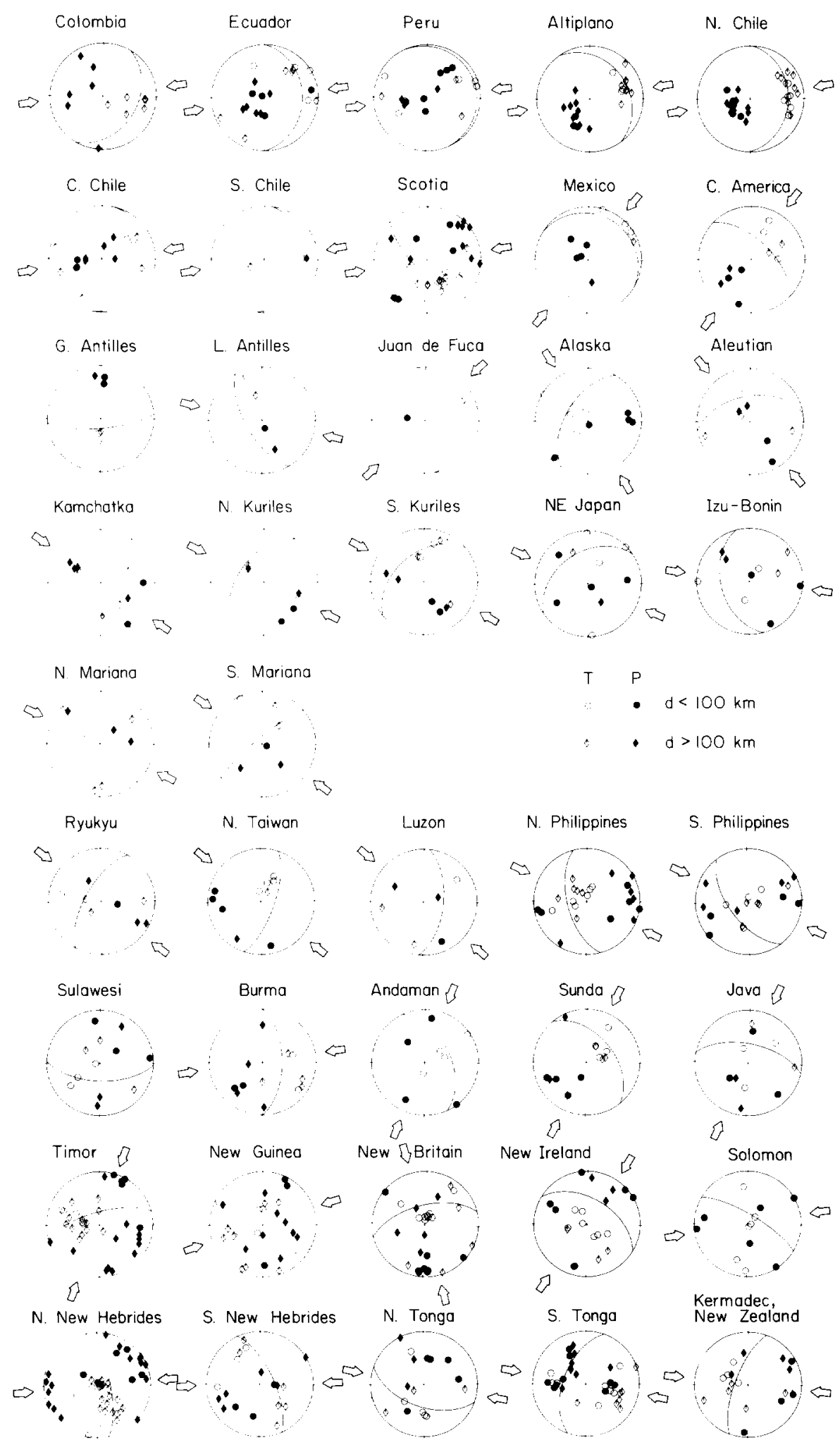
vertical but are not aligned with the subducted plate.

The Sulawesi region is a complex region with sparse large seismicity. In the Burma subduction zone continental collision is occurring; however, intermediate-depth events may be located within the attached oceanic lithosphere. Tension and compression axes are separated in the diagram but are not easily related to either the seismic zone or the convergence direction in the region. Events that occurred in the Andaman region are all $<100$ $\mathrm{km}$ depth, but the stress axis distribution is mixed. Most of the events in the Sunda trench have down-dip tensional axes, which are nearly vertical. This observation is consistent with a weakly coupled interplate boundary. The stress axes of intermediate-depth events in the Java region are mixed. In the Timor subduction region most earthquakes have nearly down-dip tensional axes that are aligned in the direction perpendicular to the convergence direction of the subducting and overriding plates.

The New Guinea region has mixed stress axes. Most events shallower than $100 \mathrm{~km}$ that occur in the New Britain region have near vertical tension axes; however, the tension axes of events with depths between 100 and $200 \mathrm{~km}$ are nearly horizontal. This pattern reflects the complexity of this region. The New Ireland region is small and very active (see Appendix 1), which may be related to high stress levels due to lateral bending of the Solomon plate beneath this arc. The stress axis distribution in the New Ireland region is mixed. Intermediate-depth events $(M \geq 6)$ in the Solomon subduction zone are shallower than $100 \mathrm{~km}$ and exhibit a mixed distribution of the stress axis.

The New Hebrides region is the most active area at intermediate depth (see Appendix 1). The trench azimuth changes from north to south, so the events have been divided accordingly. Most events in the northern segment have nearly hori- zontal compressional axes; the tensional axes of the events located below $100 \mathrm{~km}$ depth (open diamonds) are aligned with the plate. The stress axis distribution in the southern New Hebrides segment is mixed.

The Tonga subduction zone is also very active at intermediate depths. The area is divided into two regions that separate the events which occurred near the northern bend from those in the more linear section of the trench. Most of the southern events have down-dip compressional axes with the exception of a few shallower events, which are discussed in detail below. The Kermadec-New Zealand diagram shows a mixed distribution of the stress.

\section{Regional examination of intermediate-depth events}

Although the regional stress field of intermediate-depth events inferred from the focal mechanism catalog in Table IV shows that a large number of events have down-dip or nearly vertical tension axes, as discussed above (Fig. 10), many regions show a mixed or complex distribution of the tension and compression axes.

We wish to evaluate these variations in greater detail, to appraise the relative importance of static versus dynamic stress fields. Figure 11 shows the location of the intermediate-depth earthquakes with $M \geq 6.0$, which occurred from 1960 to 1984 . Boxes enclose the regions shown in Figs. 12-19. On the regional maps the plate names, bathymetric features and relative motion between adjacent plates are indicated. Holocene volcanoes are shown as open triangles and filled triangles represent volcanoes active in the last 1000 years (Moore, 1982). The fault parameters of the events in each regional figure and the event number, shown next to the lower hemisphere projection of the focal

Fig. 10. Equal area lower hemisphere projection showing $P$ (filled symbols) and $T$ (open symbols) for events in the regions listed in Table 1.3. The curve indicates the trench azimuth and the dip of the seismic zone at intermediate depth. Open arrows show the direction of convergence across plate boundaries for each region. Diamonds are symbols for events deeper than $100 \mathrm{~km}$ and circles for shallower events. 


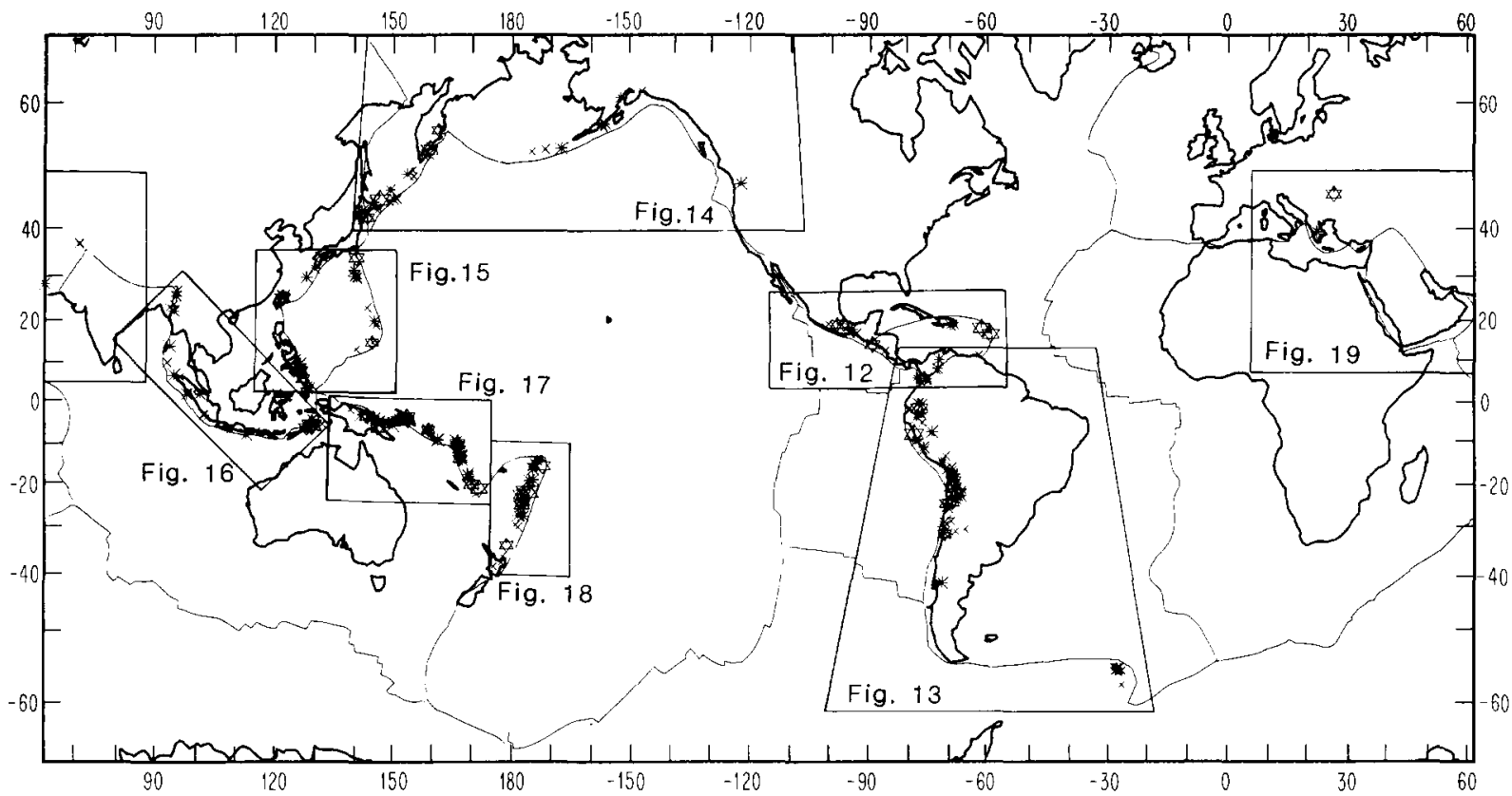

Fig. 11. Location of intermediate-depth earthquakes $(40-200 \mathrm{~km})$ with $M \geq 6.0$ that occurred from 1960 to 1984 . Boxes enclose the regions shown in Figs. 12-19.

sphere, are given in Table IV. Dark quadrants are compressional, and numbers within parentheses indicate the event depth.

In the region by region discussion that follows we describe the characteristics of the downgoing plate as well as those of the interplate boundary. Then we examine individual events or groups of nearby events relative to their location to local physical changes in the subduction zone, for example, bends or tear of the downgoing plate or subduction of topographic highs. Detailed seismicity profiles and modeling by Yamaoka et al. (1986) and Burbach and Frohlich (1986) for each subduction zone help us locate the regions where the plate is bending or tearing. Then we explore the relative location, both in space and time, of intermediate-depth events that may be related to the occurrence of large thrust events at the interplate boundary.

\subsection{Middle America}

North of the Tehuantepec Ridge the Rivera and Cocos plate subduct with a shallow dip $\left(10-20^{\circ}\right)$ beneath the North America plate (Fig. 12 ). The seismicity extends eastward to $\sim 150 \mathrm{~km}$ depth. The chain of active volcanoes forms an angle with the trench and is located farther inland than in most subduction zones. Large shallow underthrusting earthquakes with rupture lengths of $\sim 100 \mathrm{~km}$ and short recurrence intervals, $30-80$ years (Singh et al., 1981) are characteristic of the Mexican subduction zone, suggesting strong to moderate coupling along this interplate boundary. Three large intermediate-depth events occurred from 1960 to 1984 down-dip of future large subduction events. The 1964 event (39) occurred down-dip of the 1979 Petatlán earthquake aftershock area. Similarly, the 1980 Huajuapan event (248) occurred down-dip of the 1982 Ometepec doublet, and the 1973 Orizaba earthquake (178) was located down-dip of the 1978 Oaxaca aftershock zone. Although these events are consistent with the dynamic model for strongly or moderately coupled regions presented earlier, no large compressive intraplate events have occurred after these large subduction earthquakes. This suggests that the displacement at the interplate 


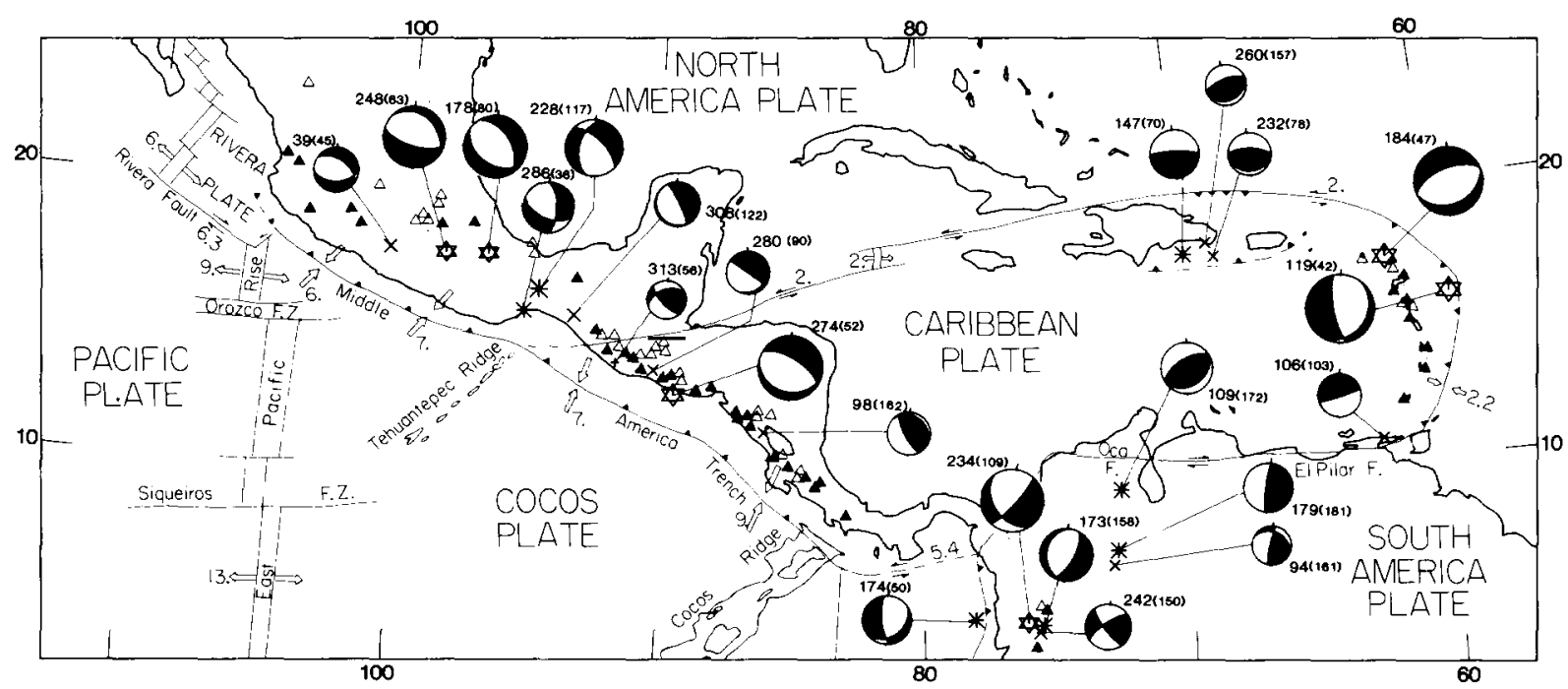

Fig. 12. Location and focal mechanisms for events with $M \geq 6.0$ that occurred in the Middle America subduction zone and in the Greater and Lesser Antilles in the Caribbean during the period of 1960-1984. Numbers refer to events in Table IV and those in parentheses indicate event depth in $\mathrm{km}$. Plate names, bathymetric highs and trench names are indicated in the figure. Open triangles are locations of Holocene volcanoes and filled triangles are recent active volcanoes (Moore, 1982). Open arrows are convergence rates (Table I) and double arrows indicate strike-slip motion across plate boundaries. The focal spheres are lower hemisphere projections, with compressional quadrants darkened. The sizes of the focal mechanisms are proportional to the magnitude.

boundary may not be large enough to change the stress characteristics at depth or that any such down-dip intraplate compressional events may be too small to identify. González-Ruiz (1986) documented the occurrence of normal faulting events down-dip of the Ometepec region within a few years before four consecutive thrusting episodes. He suggested that the occurrence of normal faulting events at intermediate depth may be an integral part of the earthquake cycle in the Ometepec region. However, the 1931 normal fault event studied by Singh et al. (1985) was located at 45 $\mathrm{km}$ depth down-dip of the region broken in 1928 by four large shallow thrust events offshore Oaxaca. Singh et al. (1985) suggested that this event may have broken the lithosphere, decoupling the Cocos plate from the overriding continental plate. Events 228 and 286 may be associated with subduction of the Tehuantepec ridge, where the arc junction is identified by Yamaoka et al. (1986). It is not known whether large interplate events occur where the Tehuantepec ridge enters the trench.
South of the Tehuantepec ridge, the Cocos plate subducts beneath the Caribbean plate with a considerably larger dip angle, which varies from $30^{\circ}$ beneath Guatemala to $65^{\circ}$ beneath Nicaragua. The seismicity is continuous to a depth of $250 \mathrm{~km}$ along Central America, but shallows toward the southeast, where a shallower dip is also observed. All events in this region are down-dip tensional. However, events 98 and $313(M \approx 6)$ have reverse focal mechanisms that suggest, under the classification of large events discussed earlier, an uncoupled interplate boundary. McNally and Minster (1981) observed low seismic slip along Central America, indicating either weak coupling or longer recurrence intervals along this plate boundary. The largest intermediate-depth event is the 1982 El Salvador earthquake (event 274), which occurred down-dip of a region where large events last occurred at the turn of the century (Astiz and Kanamori, 1984). If this region is at least moderately coupled, this event may indicate down-dip loading preparatory to a large subduction earthquake; however, the mechanisms of the historic 
events are not known, so the seismic potential is difficult to evaluate.

\subsection{Atlantic island arcs}

\subsubsection{Caribbean}

Subduction of old Atlantic oceanic lithosphere beneath the Caribbean along the Lesser Antilles trench occurs at $\sim 2 \mathrm{~cm} \mathrm{a}^{-1}$ in a westerly direction. However, as the trench curves to an E-W trend, along the Greater Antilles, the North America and Caribbean plate relative motions become very oblique and convergence reduces to $\sim 0.2 \mathrm{~cm} \mathrm{a}^{-1}$ (Fig. 12). Several studies (e.g., Stein et al., 1982; Sykes et al., 1982; Yamaoka et al., 1986) indicate that the downgoing slab curves continuously beneath the Caribbean plate. McCann and Sykes (1984), on the other hand, suggested tearing of the plate based on the focal mechanism of the 1974 earthquake $\left(M_{\mathrm{S}}=7.5\right.$, event 184); however, this event is also consistent with in-plate tensional stresses caused by the lateral bending of the lithosphere. The 1969 Christmas Day earthquake ( $M_{\mathrm{S}}=7.5$, event 119) location near the trench axis indicates lithospheric faulting in response to the slab pull in a weakly coupled region (McCann and Sykes, 1984), in agreement with the model shown in Fig. 8. Note also that this event had a relatively large number of aftershocks with respect to other intermediatedepth events. These indicate faulting along the steeply dipping plane (Stein et al., 1982). Event 106 indicates tear faulting. Several large intraplate earthquakes are located along the Lesser Antilles arc (Table $\mathrm{A}$ ); however, no large subduction events have occurred there during this century (McCann and Sykes, 1984).

In the Greater Antilles region the 120-Ma-old oceanic plate subducts along the Puerto Rico trench. At intermediate depth the downgoing lithosphere is nearly vertical (Frankel et al., 1980). Moderate intraplate earthquakes in this area (147, 232, 260) are down-dip tensional events, consistent with the model for a weakly coupled region. In this model the dip of the slab is steep and the slab's weight induces tensional stresses at intermediate depth. Frankel (1982) reports that smaller magnitude events in this region are also down-dip tensional.

\subsubsection{Scotia arc}

Tectonic studies of the Scotia arc region (Fig. 13) indicate that back-arc spreading is occurring (Barker, 1970, 1972) and that the 70-Ma-old, oceanic lithosphere of the South America plate gets younger to the south $(<10 \mathrm{Ma})$ as it subducts beneath the oceanic floor of the recently formed Sandwich plate (Frankel and McCann, 1979). The southern portion of the plate is less active and the focal mechanism of event 93 indicates down-dip compression; however, in the northern segment most events are down-dip tensional (see Fig. 13). The change from predominantly down-dip tensional to down-dip compressional stresses along the Scotia arc was also observed for focal mechanisms of smaller magnitude events by Forsyth (1975), who explains this change as the result of reduced negative buoyancy forces in the younger, southern half of the subducted plate. Evidence of tearing of the downgoing plate in the northern segment is given by some earthquake focal mechanisms (e.g., events 159, 328; Forsyth, 1975) and a gap in seismicity is observed at intermediate depth (Yamaoka et al., 1986). Events 266 and 269, which occurred within three months of each other, have very different focal mechanisms and may reflect internal deformation of the subducting plate. Several very large intraplate events have occurred in the Scotia arc in this century (Table A). The most recent event on May 26, 1964 (36), was studied in detail by Abe (1972a), who obtained $M_{\mathrm{W}}=7.8$ (from surface-wave seismic moment of $6.2 \times 10^{27} \mathrm{dyn} \mathrm{cm}$ ). The 1964 event, which is the largest recent event in this region, is consistent with the model described above for weakly coupled regions. No large $(M>7.5)$ subduction thrust events have occurred in this region.

\subsection{South America}

The Nazca plate subducts eastward along the curving margin of western South America for $\sim 5000 \mathrm{~km}$. The major topographic features being subducted along this oceanic-continental plate 


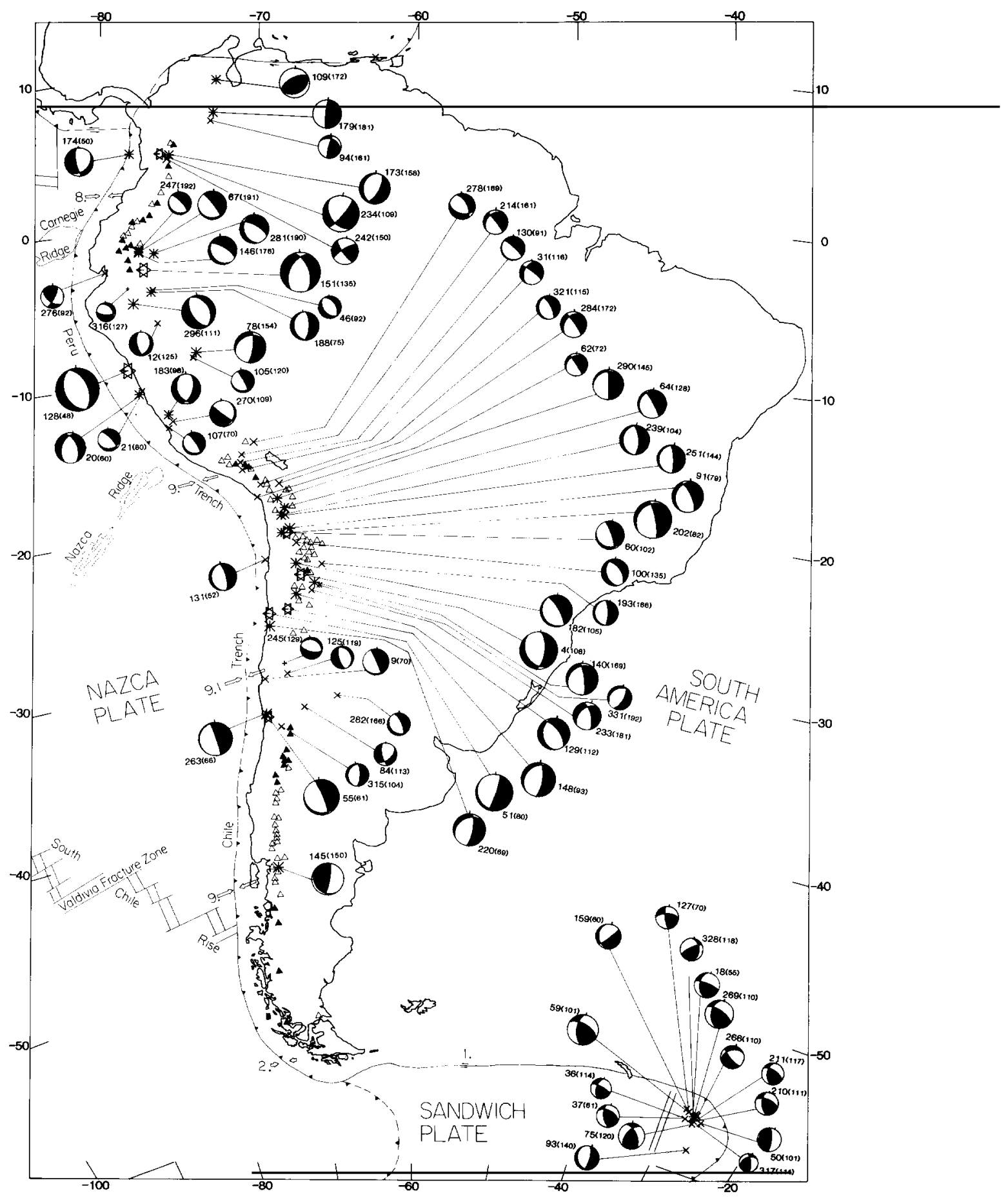

Fig. 13. Epicenters and focal mechanisms of intermediate-depth earthquakes with $M \geq 6.0$ that occurred from 1960 to 1984 (Table IV) in South America and the Scotia arc. For symbols, see Fig. 12 
boundary are the Chile rise and the Nazca and Carnegie ridges (Fig. 13). The characteristics of the downgoing lithosphere vary along the trench, defining several segments (Table I, Fig. 2). The absence or presence of the volcanic chain correlates with dip of the intermediate-depth seismicity (Barazangi and Isacks, 1976). Although intraplate seismicity is high in South America, inaccurate earthquake hypocenters (owing to poor station coverage) allow different interpretations of the configuration of the downgoing Nazca plate (Chin and Isacks, 1983). For example, Barazangi and Isacks (1976) and Yamaoka et al. (1986) propose a tear in the subducted lithosphere, where the slab dip changes abruptly from $8^{\circ}$ to $28^{\circ}$ along the Peru-Altiplano border line. In contrast, Hasegawa and Sacks (1981) suggest that the Nazca plate is contorted rather than torn in this region, based on observations from local seismic data. The observed continuous seismicity is as deep as $300 \mathrm{~km}$ for all regions except Southern Chile; however, events deeper than $500 \mathrm{~km}$ are observed only in Colombia, Peru and Northern Chile regions (Fig. 13). These deep events probably occur within a detached segment of the subducted lithosphere (Isacks and Molnar, 1971; Stauder, 1975). Most intermediate-depth events in South America (from Colombia to Central Chile, Fig. 10) have nearly down-dip tensional axis induced by the lithosphere's negative buoyancy, or possibly in response to continental loading.

We divide the following discussion into five sections, corresponding to variations of intermediate-depth seismicity and occurrence of recent large subduction earthquakes along the Nazca-South America interplate boundary.

\subsubsection{South Chile}

Seismicity in this $1250-\mathrm{km}$-long section extends to a depth of $160 \mathrm{~km}$ with a shallow dip. The shallow activity is dominated by the aftershocks of the 1960 Chile $\left(M_{\mathrm{W}}=9.5\right)$ earthquake. This region, from $34^{\circ}$ to $45^{\circ} \mathrm{S}$, is considered to be the end member case of a very strongly coupled subduction zone (Uyeda and Kanamori, 1979). Relatively young, 0-35 Ma, oceanic lithosphere is being subducted at $-9.5 \mathrm{~cm} \mathrm{a}^{-1}$. Large intraplate (intermediate-depth and outer-rise) earthquakes that occurred in this region during this century have been discussed by Astiz and Kanamori (1986). We should remark that the down-dip compressional event (145) that occurred on May 8, 1971 is the only earthquake with $M \geq 6$ to have occurred to date in this region at intermediate depth. Astiz and Kanamori (1986) interpret this event, along with the extensive sequence of outer-rise tensional events following the 1960 rupture (Christensen and Ruff, 1987) as a temporal change in the intraplate stress environment induced by the interplate event. Large down-dip tensional events preceded the 1960 thrust event in 1934 and 1949, indicating that the down-dip edge of the future thrust zone was loaded by tensional stresses induced by slab pull prior to the interplate failure. The remaining $250-\mathrm{km}$-long section in Southern Chile to the north of the 1960 rupture was broken by the $1939\left(M_{\mathrm{S}}=7.8\right)$ and the $1928\left(M_{\mathrm{S}}=8.0\right)$ interplate earthquakes. From 1960 to 1984 no intermediate-depth earthquakes with $M \geq 6$ have occurred there.

\subsubsection{Central Chile}

This region $\left(29^{\circ}\right.$ to $\left.34^{\circ} \mathrm{S}\right)$ is characterized by a very shallow-dipping slab $\left(10^{\circ}\right)$ at intermediate depth, the absence of active volcanism and occurrence of large underthrusting events with rupture lengths of $\sim 300 \mathrm{~km}$. The northern half is defined by the aftershock area of the 1906 Valparaiso ( $M_{\mathrm{S}}=8.4$ ) thrust earthquake, which has reruptured over $\sim 75 \%$ of its length, in two large subduction events that occurred on July $9,1971\left(M_{\mathrm{S}}\right.$ =7.5) and March 3, $1985\left(M_{\mathrm{S}}=7.8\right)$. The southern-most segment appears to be a seismic gap (Korrat and Madariaga, 1986).

Stress axes of intermediate-depth events in this region are aligned with the convergence direction (Fig. 10); however, the tension axes of most events (except events 245, 125 and 84) dip at $-30^{\circ}$ instead of $10^{\circ}$, as inferred by the intraplate seismicity in the region (Fig. 13). Events 125 and $245(M \approx 6$ ) occurred near the northern limit of the 1943 aftershock zone. Their focal mechanisms indicate internal deformation of the plate and slab segmentation associated with the increase in plate dip $\left(30^{\circ}\right)$ to the north, as proposed by Isacks and Barazangi (1977). Event $9\left(m_{\mathrm{b}}=6.3\right)$ occurred in 
1963, down-dip of the 1943 thrust event rupture area, suggesting that by this time the interplate boundary was at least moderately coupled, and normal faulting events could occur at the base of the coupled zone. Further investigation is required to determine whether a change in the stress field orientation of intermediate-depth events occurred down-dip of the 1943 rupture area.

Before the occurrence of the recent 1971 and 1985 Valparaiso earthquakes, two normal faulting events occurred down-dip at intermediate depth in 1965 (55) and in 1983 (315), respectively, in agreement with the dynamic model for coupled regions (Fig. 3b). Malgrange et al. (1981) studied the 1965 and 1971 Aconcagua events in detail. In 1981, a large normal faulting event $\left(263, M_{\mathrm{W}}=7.0\right)$ occurred down-dip of the 1971 aftershock zone. Although the occurrence of smaller $(M<6)$ downdip compressional events cannot be ruled out, the occurrence of the 1981 event (263) suggests that displacement at the intraplate boundary was not large enough to change the stress field orientation at depth. Note also that in 1983 a compressional outer-rise event occurred offshore the future 1985 Valparaiso earthquake rupture zone (Christensen and Ruff, 1983). Thus, this region agrees with the dynamic model shown in Fig. $3 b$ in that an outerrise compressional event and a down-dip tensional event occurred before the thrust event. However, neither a large tensional outer-rise nor a down-dip compressional intermediate-depth event has occurred to date. One possibility is that the displacement at the interplate boundary may not be large enough to change the stress orientation, but may be sufficient to decrease temporarily the tensional intraplate seismicity in the region down-dip from the large thrusting earthquake.

\subsubsection{Altiplano and North Chile}

The downgoing lithosphere dips $\sim 30^{\circ}$, bending gently along the coasts of southern Peru and northern Chile. Note that the volcanic chain reappears (Fig. 13) and that since at least 1922 no large thrust earthquakes have occurred along the entire length of the interplate boundary (Kelleher et al., 1974; McCann et al., 1979). These regions have been very active at intermediate depth, not only during the 1960-1980 time period but throughout this century (Appendix 1). All of the events in Fig. 13, from event 278 to the northern boundary of the Altiplano region at event 220 to the south boundary of the North Chile region, have down-dip tensional axes. The compression axes are oriented perpendicular to the downgoing plate with a nearly vertical plunge, suggesting that continental loading may play an important role in the stress distribution at intermediate depth, given that the Andes are much broader in the Altiplano-North Chile region. Note also the azimuthal variation of the tension axis along the gently bending Nazca plate, from $-\mathrm{N} 40^{\circ} \mathrm{E}$ for event 278 to $\sim \mathrm{N} 120^{\circ} \mathrm{E}$ for event 220 . The focal mechanisms of intermediate-depth events in this region also agree with the model for a coupled interplate boundary, since normal faulting events occur at the base of the coupled region in response to down-dip pull of the subducting lithosphere.

Christensen and Ruff (1987) report two compressional outer-rise events offshore the 1922 aftershock area $\left(26^{\circ}\right.$ to $\left.29^{\circ} \mathrm{S}\right)$ in 1964 and 1969. The earlier event was followed by a $M_{\mathrm{S}}=7.4$ thrust event that occurred on October 4, 1983. Malgrange and Madariaga (1983) documented the variation of focal mechanisms of moderate intermediate-depth events associated with the large $1966\left(M_{\mathrm{W}}=7.7\right)$ thrust event $\left(25^{\circ}\right)$. A large down-dip tensional event preceded the thrust on February 23, 1965 (51), and a small down-dip compressional aftershock occurred at the northern end of the rupture zone on June 21, 1967. They interpreted their observations as possible evidence of the presence of a double seismic zone in the region. An alternative interpretation is that these events reflect the temporal change in coupling at the thrust boundary as shown in Fig. 3b.

\subsubsection{Ecuador-Peru}

The Ecuador-Peru region, extending from $0^{\circ}$ to $-16^{\circ} \mathrm{S}$, is bounded to the north and south by the Carnegie and Nazca ridges (Fig. 13). This region, albeit complex, offers a good example of the relationship between the coupled interplate boundary and intraplate seismic activity. Seismicity along this region dips at $\sim 30^{\circ}$ at shallow depth, while at $\sim 100 \mathrm{~km}$ depth it becomes almost horizontal, extending laterally almost $300 \mathrm{~km}$ 
(Barazangi and Isacks, 1979). The shallow dip ( $\approx 10^{\circ}$ ) of the subducting lithosphere at intermediate depth has been associated with the lack of volcanism (Fig. 13) by Barazangi and Isacks (1976). Two regions can be defined in terms of the absence or presence of large thrust events at the interplate boundary. The northern segment has no known historic large subduction events and extends from $0^{\circ}$ to $10^{\circ} \mathrm{S}$ (McCann et al., 1979). The oceanic lithosphere in this region is heterogeneous with the Carnegie ridge subducting from $0^{\circ}$ to $3^{\circ} \mathrm{S}$, and numerous fractures are present farther south, seaward from the trench. The second region, extending from $10^{\circ}$ to $16^{\circ} \mathrm{S}$, has active intraplate thrust activity.

A cluster of events $(247,67,28,146)$ at the northern edge of the subducting Carnegie ridge indicates tear faulting, with one nodal plane almost perpendicular to the trench that may be associated with subduction of the presumably more buoyant lithosphere (Barazangi and Isacks, 1976). Events 151, 46, 188, 296 and 12 are down-dip tensional, consistent with a strongly coupled interplate. Christensen and Ruff (1987) reported outer-rise compressional events in this region. Event $316(M \approx 6)$ may be associated with internal deformation of the Nazca plate, resulting from the presence of the Carnegie ridge to the north. The compression axes of event 276 are almost horizontal, suggesting that high compressive stresses induced by the subducting ridge may be dominant to $\sim 100 \mathrm{~km}$ depth. However, this event could be explained as a result of bending of the downgoing slab as it flattens at intermediate depth (see fig. 8 in Isacks and Barazangi, 1977).

The southern segment, from $10^{\circ}$ to $\sim 16^{\circ} \mathrm{S}$, is characterized by large thrust events with rupture lengths of $\sim 150 \mathrm{~km}$. The most recent sequence occurred from south to north in 1942, 1974, 1940 and 1966 (e.g., McCann et al., 1979). Along the southern boundary of this region several studies have proposed a major tear of the subducting lithosphere (Isacks and Barazangi, 1977; Yamaoka et al., 1986) to accommodate the sharp increase in the dip angle of the downgoing plate farther south. Note that from 1960 to 1984 no large tearing events occurred in this region at intermediate depth, which favors Hasegawa and Sacks' (1981) alternate interpretation of continuous slab contortion in this region. Also, no large events $(M \geq 6)$ have occurred in this same time period down-dip of the 1940 and 1942 aftershock zones.

Before the large thrust earthquake of October $1974\left(M_{\mathrm{W}}=8.1\right)$ two down-dip tensional events occurred. In 1968 (107) and in January 1974 (183, $m_{\mathrm{b}}=6.6$ ) both events were located down-dip of the future 1974 thrust rupture zone. In 1982 (270), a down-dip compressional aftershock took place near the earlier 1968 tensional event. These observations agree with the dynamic model shown in Fig. 3b. However, no large outer-rise events occurred either before or after the large 1974 thrust earthquake (Christensen and Ruff, 1987). Downdip of the $1966\left(M_{\mathrm{W}}=8.1\right)$ earthquake aftershock area two down-dip tensional events were recorded in $1963(20,21)$, and no large intraplate activity has occurred since the 1966 event.

The largest intraplate earthquake in this region occurred on May 31, $1970\left(128, M_{\mathrm{W}}=7.8\right)$, and this event has been studied by several investigators (Abe, 1972b; Stauder, 1975). This event is unusual in many ways. For instance, it is located between the regions of active thrusting and apparent aseismic subduction along Peru. Also, a large number of small aftershocks (see Fig. 10) followed this event, defining a steeply dipping fault plane that perhaps broke the entire subducting lithosphere (Abe, 1972b). Some of these events have normal faulting mechanisms while others exhibit compressional mechanisms (Dewey and Spence, 1979). Christensen and Ruff (1987) reported that near the northern edge of the 1966 thrust event an outer-rise compressional event occurred in September 1967. Note also that two normal-faulting events $(78,105)$ occurred down-dip prior to the large 1970 normal-fault earthquake. The occurrence of these events, along with the tensional $1970\left(M_{\mathrm{W}}=7.8\right)$ earthquake, may reflect the presence of a strongly coupled interplate boundary. Abe (1972b) suggests that the 1970 event occurred in response to the gravitational pull exerted by the denser sinking slab.

\subsubsection{Colombia}

The strongly coupled interplate boundary north of the Carnegie ridge, from $5^{\circ} \mathrm{N}$ to $0^{\circ}$, has very 
young oceanic lithosphere subducting along the shallow Colombia trench at $\sim 8 \mathrm{~cm} \mathrm{a}^{-1}$. The seismicity dip is small at shallow depth and increases to $35^{\circ}$ at intermediate depth. The volcanic chain parallels the coast with numerous active volcanoes (Fig. 13). The entire length of the earlier 1906 Colombia $\left(M_{\mathrm{S}}=8.7\right)$ earthquake has been reruptured by the recent thrust events, which progressed from south to north, in 1942, 1958 and 1979 (Kelleher, 1972; Kanamori and McNally, 1982; Beck and Ruff, 1984).

Events 109, 179 and 94 are located in northern Colombia where the trench is not well defined. These events are consistent with down-dip tensional stresses that are probably induced by the negative buoyancy of the sinking slab. No large $(M \geq 6)$ intermediate-depth events are located down-dip of the 1942 and 1958 aftershock zones during the period of 1960 to 1984 . However, near the northern edge of the 1979 aftershock zone several events have occurred. The shallowest event (174), which took place in 1973, is located near the trench axis. It indicates outer-rise tensional stress in the segment adjacent to the 1979 rupture, perhaps indicative of lateral loading of the locked zone by aseismic slip to the north. Down-dip of the northern end of the December $1979\left(M_{\mathrm{W}}=8.2\right)$ thrust event rupture zone, two normal-fault events $(173,234)$ at intermediate depth preceded the interplate rupture. Event 234 took place in November $1979\left(m_{\mathrm{b}}=7.2\right)$, a few weeks before the large thrust event. Although event 242, which followed the 1979 event, is down-dip tensional, the focal mechanism indicates tear faulting, perhaps due to slab segmentation. An outer-rise tensional event seaward of the 1979 rupture zone occurred on January 1981 (Christensen and Ruff, 1987) indicating that the thrust event was large enough to put the outer rise into tension. No precursory outer-rise compressional events were recorded.

\subsection{North Pacific}

\subsubsection{Cascades}

Shallow seismicity is almost non-existent along the $500-\mathrm{km}$-long plate boundary between the Juan de Fuca plate's young oceanic lithosphere and the
North America plate (Fig. 14). However, seismicity at intermediate depth defines a plate dipping at $\sim 22^{\circ}$ extending to $100 \mathrm{~km}$ depth (Crosson, 1980). Heaton and Kanamori (1984) infer a strongly coupled interplate boundary along the Juan de Fuca plate. The occurrence of the 1965 $\left(57, m_{\mathrm{b}}=6.9\right)$ and $1949\left(m_{\mathrm{h}}=7.1\right)$ Puget Sound earthquakes, both down-dip tensional normalfaulting events (Langston and Blum, 1977) at intermediate depth, also suggest a strongly coupled interplate boundary according to the classification in Fig. 8 .

\subsubsection{Alaska-Aleutians}

Convergence between the North American and Pacific plates takes place along the Alaska-Aleutian trench in a northwest direction (Fig. 14). The oceanic plate becomes progressively older to the west along the trench, ranging from 40 to $65 \mathrm{Ma}$. Seismicity defines the downgoing slab to a maximum depth of $280 \mathrm{~km}$ underneath the Aleutian Arc. The dip of the subducting lithosphere shallows progressively to the east from $65^{\circ}$ underneath the Aleutian Islands to $\sim 25^{\circ}$ beneath the Cook Inlet, where seismicity extends to only 150 $\mathrm{km}$ depth. Under the Rat Island arc, west of the Bowers ridge, the maximum seismicity depth is only $100 \mathrm{~km}$, and the arc turns into a transform boundary as it approaches the Kamchatka coast. The volcanic chain that parallels the trench from Alaska to the Rat Island arc is very active (Fig. 14).

The 1964 Alaska $\left(M_{\mathrm{W}}=9.2\right), 1957$ Aleutian $\left(M_{\mathrm{W}}=9.1\right)$ and 1965 Rat Island $\left(M_{\mathrm{W}}=8.7\right)$ earthquakes, with rupture lengths of over $500 \mathrm{~km}$, are among the largest thrust events in this century (Sykes, 1971). However, only a few large $\left(m_{\mathrm{B}}>7\right)$ intermediate-depth events have occurred in the Aleutian-Alaska region (see Table A). Event 306 $\left(m_{\mathrm{b}}=6.3\right)$ is located near the northern edge of the 1964 Alaska earthquake aftershock area. This event shows in-plate horizontal tension and a vertical compression axis, which suggests that it may be associated with either deformation in the subducting plate or with continental loading. Only one event with $M \geq 6$ has occurred down-dip of the 1964 Alaska aftershock zone, on December 1968 $\left(110, m_{\mathrm{b}}=6.5\right)$. This event had many aftershocks 


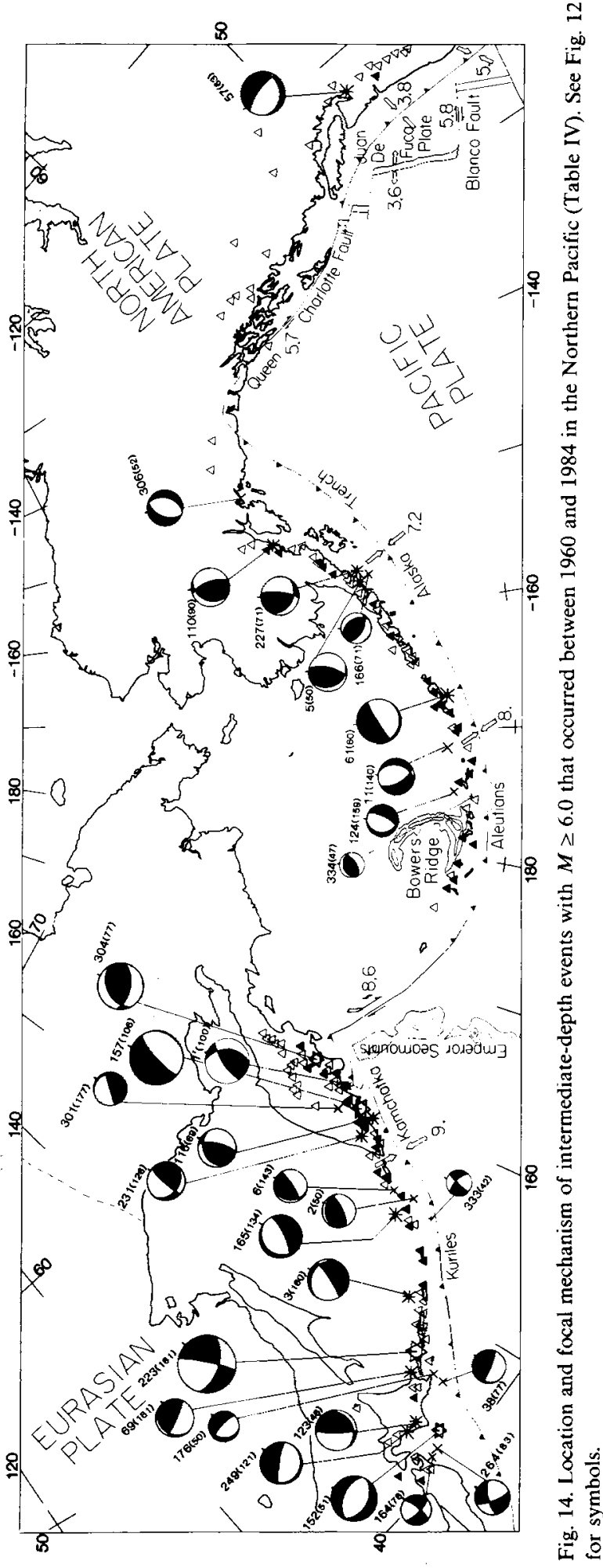

and may be associated with the tear in this region proposed by Burbach and Frohlich (1986). Only one outer-rise tensional event was detected after the 1964 rupture (Christensen and Ruff, 1987), and no outer-rise compressional events are known to have occurred in the region.

Events 5, 166 and 227 occurred down-dip of the northern edge of the 1938 Alaska earthquake aftershock zone (McCann et al., 1979). Hudnut and Taber (1987), using local seismicity data in this region (known as the Shumagin gap), observed a change in the intraplate seismicity pattern, from a single seismic zone, where events 5 , 166 and 227 occur, to a double seismic zone to the west where no larger intermediate-depth events have been observed after 1960. They suggested that this difference may reflect a stress change at the interplate boundary between these two regions that have either different aseismic-to-seismic slip ratios, or are at different stages in the earthquake cycle.

Two down-dip tensional events, 61 and 334, have occurred down-dip of the 1957 Aleutian earthquake aftershock zone. Note that event 334 , which occurred in 1984 was located down-dip of the 1986 Andreanof Islands $\left(M_{\mathrm{W}}=8.0\right)$ earthquake aftershock zone. Before the 1957 Aleutian event, three large $\left(m_{\mathrm{B}}>7\right)$ intermediate-depth earthquakes occurred within the 20 years prior to this event, whereas only one large event $\left(61, m_{\mathrm{B}}=\right.$ 6.9) has occurred thereafter (Table A). This suggests that displacement from the 1957 event at the interplate boundary may have only decreased the tensional down-dip stresses at intermediate-depth. Deeper events (11 and 124), however, show a down-dip compressional axis and a near horizontal in-plate tensional axis, which could be related to lateral bending or segmentation of the downgoing plate (Stauder, 1968, 1972; Spence, 1977). Christensen and Ruff (1987) discuss the extensive sequence of outer-rise tensional events that followed the 1957 and 1965 thrusts.

\subsection{Western Pacific}

The 95-Ma-old Pacific plate subducts westward under the Eurasia plate at $-9 \mathrm{~cm} \mathrm{a}^{-1}$ along the Kamchatka-Kurile-Japan trench (Fig. 14). To the 
south, as the Pacific plate increases in age to 135 $\mathrm{Ma}$, it subducts along the Izu-Bonin-Mariana trench system beneath the Philippine plate with a slower convergence rate and seismicity, defining a nearly vertical plate (Katsumata and Sykes, 1969). The chain of active volcanoes parallels the trench axis for most of its length as shown in Figs. 14 and 15. The dip angle and extent of the intraplate seismicity vary along the trench as indicated in Table I. Double seismic zones at intermediate depth have been reported in the Kurile-Kamchatka region (see fig. 5 of Stauder and Maulchin, 1976) and in Japan (Hasegawa et al., 1979). The strength of coupling at the interplate boundary, as inferred from earthquake magnitudes of thrust events (Ruff and Kanamori, 1980), changes along the trench strike, from strong coupling offshore the Kamchatka Peninsula to seismic decoupling along the Mariana trench.

\subsubsection{Kamchatka}

The 1952 Kamchatka $\left(M_{\mathrm{W}}=9.0\right)$ earthquake broke the southern $400 \mathrm{~km}$ of the interplate boundary along the peninsula. To the north, the $1959\left(M_{\mathrm{S}}=7.8\right)$ and the $1923\left(M_{\mathrm{W}}=8.5\right)$ events ruptured the remaining $150 \mathrm{~km}$. Intermediate-depth earthquakes under Kamchatka can be divided into two depth groups. Events deeper than $125 \mathrm{~km}$ $(231,301)$ have down-dip compressional focal mechanisms similar to deeper events in this region (Stauder and Mualchin, 1976). The shallower events show a temporal change in stress axes' orientation, as shown in Fig. 3b. A down-dip compressional event $\left(1^{\prime}, m_{\mathrm{b}}=6.9\right)$ occurred on July 1960, indicating that displacement at the interplate boundary during the $1952\left(M_{\mathrm{W}}=9.0\right)$ and $1959\left(M_{\mathrm{S}}=7.8\right)$ events induced compressional stresses at intermediate depth. After July 1969, three down-dip tensional events $(116,157$, 304) with $m_{\mathrm{b}} \geq 6.5$ ) occurred at similar depth, suggesting that the interplate boundary is at least moderately coupled once again. Moreover, Christensen and Ruff (1987) remarked that in the outer-rise region, just after the 1952 Kamchatka earthquake, tensional events occurred. However, compressional events have been observed offshore southern Kamchatka, indicating that coupling at the interplate boundary has allowed compressional stresses to accumulate again.

\subsubsection{Kuriles-Japan}

The interplate boundary along northeast Japan and the southern Kurile Island arc has ruptured during a recent sequence of large thrust earthquakes, which are distributed from southwest to northeast along the trench, in $1952\left(M_{\mathrm{W}}=8.1\right)$, $1973\left(M_{\mathrm{W}}=7.8\right), 1969\left(M_{\mathrm{W}}=8.2\right), 1958\left(M_{\mathrm{W}}=\right.$ 8.3) and $1963\left(M_{\mathrm{W}}=8.5\right)$ (Beck and Ruff, 1987; Schwartz and Ruff, 1987). However, along the northern $500 \mathrm{~km}$ of the Kurile trench, the last shallow earthquake occurred in $1915\left(M_{\mathrm{S}}=8.0\right)$, but it is unknown whether this event broke the Kurile gap or was possibly an intraplate event (McCann et al., 1979). Christensen and Ruff (1987) observed compressional events offshore from this gap which they interpret as evidence for strong coupling at the interplate boundary in this region. Large intermediate-depth events $\left(m_{\mathrm{b}}>6\right)$ downdip of the Kurile gap are only located close to its northern and southern boundaries, so these events may be associated, respectively, with the 1952 Kamchatka $\left(M_{\mathrm{W}}=9.0\right)$ or the 1963 Kurile Island $\left(M_{\mathrm{W}}=8.5\right)$ thrust earthquakes.

At the southern end of the 1952 Kamchatka rupture zone, event 2 has down-dip tensional mechanisms at a depth of $50 \mathrm{~km}$. Event 6 has a down-dip tensional axis, whereas event 165 has a down-dip compressional axis. The relative location of these events is consistent with a double seismic zone at intermediate depth (Stauder and Maulchin, 1976). Event $333\left(M_{\mathrm{W}}=5.6\right)$ indicates intraplate deformation near the outer rise. Event 3, which occurred in 1961 at a depth of $160 \mathrm{~km}$ down-dip of the 1963 interplate event, has a down-dip compressional mechanism. This event is not consistent with the temporal model in Fig. $3 \mathrm{~b}$.

Large intermediate-depth events that occurred from 1960 to 1984 down-dip of the Japan-southern Kurile trench reflect the complexity of the subducting lithosphere in this region (e.g., Burbach and Frohlich, 1986). Most intermediate-depth earthquakes in this region, events $223\left(m_{\mathrm{b}}=7.8\right)$, $176\left(m_{\mathrm{b}}=6.0\right), 123\left(m_{\mathrm{b}}=6.7\right), 164\left(m_{\mathrm{b}}=6.7\right)$ and $264\left(m_{\mathrm{b}}=6.3\right)$, have hinge-faulting mechanisms. Note also that events 223 and 123 had 
more aftershocks within one week than similar magnitude intermediate-depth events (see Fig. 9). Event 38, with a down-dip tensional mechanism, occurred in 1968 at the base of the $1952\left(M_{\mathrm{w}}=\right.$ 8.1) aftershock zone, indicating a coupled interplate boundary. Events 69 and 249 are down-dip tensional. Event $152\left(m_{\mathrm{b}}=7.0\right)$ has a vertical compression axis and a horizontal tensional axis, reflecting lateral bending of the downgoing plate, consistent with the orientation of stresses induced by a buckling plate (Sasatani, 1976). This complexity suggests that the magnitude of intermediate-depth stresses underneath northeast Japan owing to the distortion of the downgoing slab is greater than those induced by the interplate boundary. However, farther south along Honshu, the interplate boundary is uncoupled allowing bending stresses to concentrate near the trench axis, resulting in the great normal-faulting 1933 Sanriku $\left(M_{\mathrm{W}}=8.4\right)$ earthquake (Kanamori, 1970). Many large intermediate-depth events have occurred down-dip of the Kurile-Japan trench prior to 1960 (see Appendix 1), but the mechanisms are not known for many of these.

\subsubsection{Izu-Bonin}

Thrust earthquakes along the Izu-Bonin trench (Fig. 14) are infrequent and have maximum magnitudes of $\sim 7.4$ (McCann et al., 1979). However, many large $\left(m_{\mathrm{B}}>7.0\right)$ intraplate earthquakes have occurred during this century (Table A). Event 168 $\left(m_{\mathrm{B}}=7.4\right)$ has a horizontal compression axis aligned with the plate convergence direction and a down-dip tension axis, consistent with stresses induced by the negative buoyancy of the downgoing plate. However, event $70\left(m_{\mathrm{b}}=6.8\right)$ has near vertical compressive stresses and a horizontal tension axis. These events have similar depths, but event 168 is located closer to the trench axis, suggesting the presence of a double seismic zone in the region. Moreover, the focal mechanisms of these events are consistent with unbending of the downgoing plate. Earthquakes that occur below 80 $\mathrm{km}$ depth are down-dip compressional events or tear faults $(28,235,277)$.

Figure 9 shows that event 168 had more than 25 aftershocks with $m_{\mathrm{b}}>3$ within a week. If this event was shallower, its focal mechanism's solu- tion would be consistent with a shallow-thrust event in this region. The $\mathrm{pP}-\mathrm{P}$ phases constrained the depth of this event on the assumption that the event is not a multiple source shallow earthquake. A more detailed analysis of this event may be required to resolve its nature confidently.

\subsubsection{Marianas}

In the Mariana subduction zone, back-arc spreading is taking place, the interplate boundary is uncoupled, and shallow-thrust earthquakes are infrequent, with maximum magnitudes of $\sim 7.0$ (Ruff and Kanamori, 1980). In 1902 a large $\left(M_{\mathrm{S}}\right.$ $=7.9$ ) earthquake occurred in the Mariana Island arc. Although this event is considered a shallow event, there was no tsunami associated with it, perhaps indicating that this event was an intermediate-depth event (McCann et al., 1979). The focal mechanism of event 268 shows in-plate horizontal compression, while events 49 and 307 have in-plate horizontal tension. These variations are consistent with lateral bending of the slab along the trench strike, from convex to concave (Burbach and Frohlich, 1986). Seismicity is poorly defined in the southern Mariana arc, where events $196\left(m_{\mathrm{b}}=7.1\right)$ and $289\left(m_{\mathrm{b}}=6.1\right)$ indicate tear faulting below $100 \mathrm{~km}$ depth. Event 96 shows vertical compressive stresses and horizontal tension in the convergence direction.

\subsection{Philippine Sea}

The Philippine Sea (Fig. 15) is one of the most complex tectonic areas because the Philippine and Eurasia plates subduct in different directions in this region. The Philippine plate subducts to the northwest along the Nankai Trough and the Ryukyu trench and to the southwest along the Philippine trench. The Eurasia plate subducts eastward along the Luzon trench and along the west coast of Negros Island. The Celebes sea-floor subducts eastward along the Cotabato trench, west of Mindanao Island, and to the south along the Sulawesi trench (Cardwell et al., 1980). Seismicity in the Nankai Trough area is limited to shallow depths $(<60 \mathrm{~km})$ and the interplate boundary in this region is strongly coupled. In contrast, all other subduction zones in the Philippine Sea 


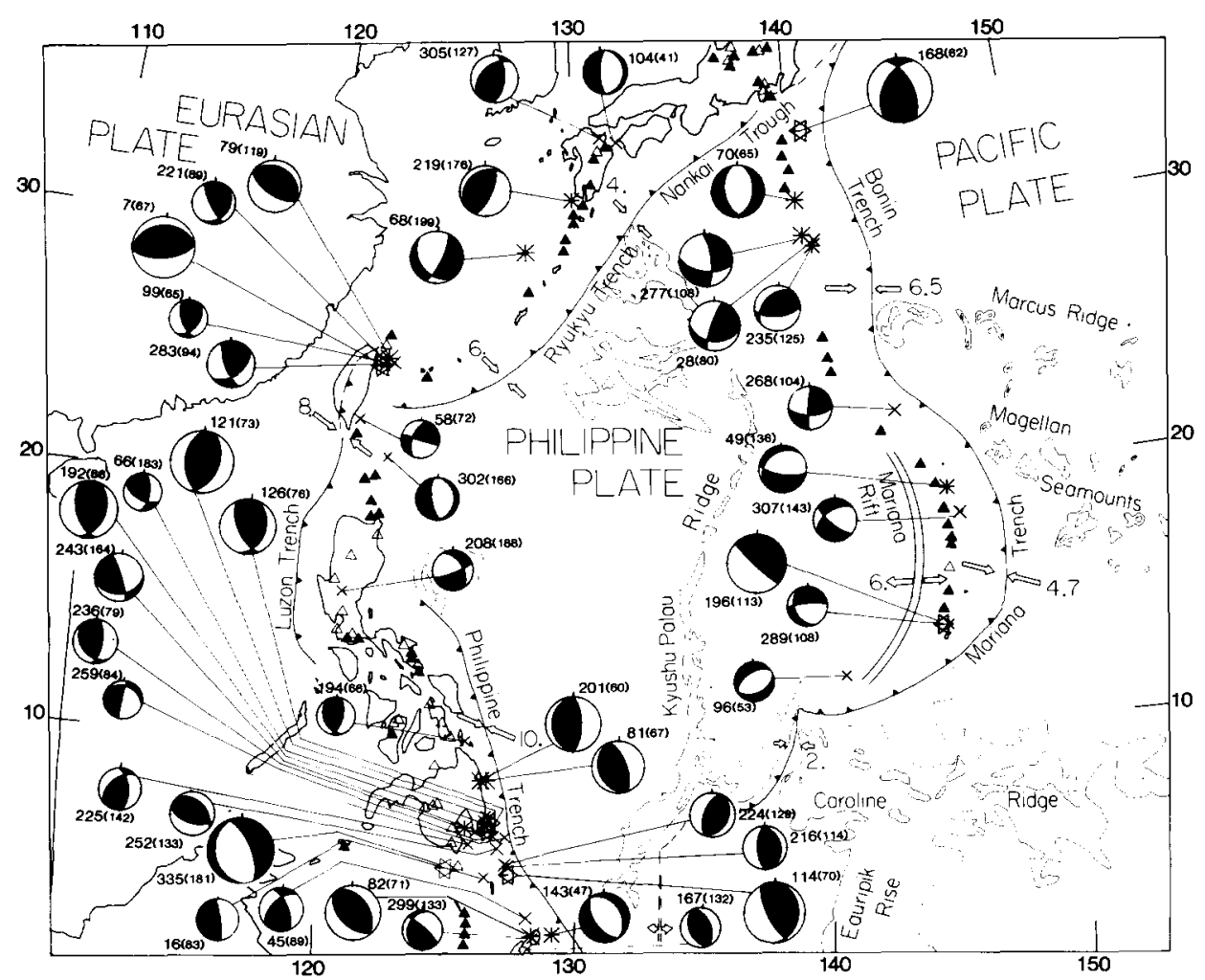

Fig. 15. Focal mechanisms and location of intermediate-depth earthquakes $(M \geq 6.0)$ associated with the Philippine plate that occurred between 1960 and 1984. For symbols, see Fig. 12.

region are weakly coupled at the interplate boundary (Ruff and Kanamori, 1980) and only tensional outer-rise events are observed there (Christensen and Ruff, 1987).

\subsubsection{Ryukyu-North Taiwan}

Many large $\left(m_{\mathrm{B}}>7\right)$ intermediate-depth earthquakes occurred in the Ryukyu-north Taiwan region before 1960 (see Appendix 1), including the largest known intermediate-depth event, $m_{\mathrm{B}}=8.1$, that occurred in 1911 under the Ryukyu Island arc. However, after 1960 this region has been relatively quiet at intermediate depth (Fig. 15). Seismicity extends to $200 \mathrm{~km}$ deep along most of the trench.

Event 104 indicates hinge faulting between the younger ocean floor that subducts in the Nankai Trough and that of the older Philippine seafloor to the west of the Kyushu-Palau ridge. Shiono et al. (1980) indicate that a marked change in the dip and state of stress of intermediate-depth seismicity is observed along the Ryukyu Island arc. North of the Tokaora channel, the slab dips at $\sim 70^{\circ}$, active volcanism is present (Fig. 15), and intermediate-depth events with $m_{\mathrm{b}} \approx 5$ have down-dip tensional mechanisms in response to the negative buoyancy of the downgoing plate. Note that events $219\left(m_{\mathrm{b}}=6.7\right)$ and $305\left(m_{\mathrm{b}}=6.5\right)$ are down-dip tensional. South of the Tokaora channel, the downgoing plate dips at $\sim 45^{\circ}$, volcanism is scarce, and focal mechanisms of $m_{\mathrm{b}} \approx 5$ events indicate down-dip compression, as is true for the large event 68 in Fig. 15. These changes could be explained in terms of lateral differences in temperature in the surrounding mantle, as reflected by volcanism, which can produce less or more resistance to the subducting lithosphere (Shiono et al., 1980).

A cluster of intermediate-depth events underneath north Taiwan delineates the edge of the 
downgoing Philippine plate. The larger events (7, $m_{\mathrm{b}}=7.2$, and $79, m_{\mathrm{b}}=6.7$ ) in this region have near vertical tensional axes that reflect the weakly coupled interplate boundary and the negative buoyancy forces exerted by the downgoing plate. Events 99, 221 and 283 are hinge-faulting events.

\subsubsection{Luzon}

Shallow and intermediate-depth seismicity in this region is scarce and not well defined (Cardwell et al., 1980). During this century only a few large earthquakes have occurred in this region (see Appendix 1). Intermediate-depth events (58, 302, 208 ) in the Luzon subduction zone may reflect only in-plate deformation. However, note that event 302 is down-dip compressional, suggesting resistance to subduction of the plate at depth.

\subsubsection{Philippines}

Cardwell et al. (1980) discussed in detail the very complex tectonic setting of the Philippine Island arc, which is bounded to the west by the shallow Negros and Cotabato subduction zones and to the east by the Philippine trench, where earthquakes occur as deep as $640 \mathrm{~km}$. Frequent large shallow and intermediate-depth earthquakes have taken place in this region during this century (see, e.g., McCann et al. (1979) and Appendix 1). In the northern section of the Philippine trench all intermediate-depth events, from events 126 to 225 in Fig. 15, have either near down-dip tensional mechanisms or vertical tension axes consistent with the model for partially coupled regions (Fig. 8 ) in which vertical tensional stresses are induced by the negative buoyancy of the subducting plate. Similarly, events along the south Philippine trench have nearly down-dip tensional axes, with the exception of the deepest event, $335 M_{\mathrm{W}}=7.5$, which has a down-dip compressional axis.

\subsubsection{Sulawesi}

The Sulawesi subduction zone (Fig. 16) was very active at intermediate depths prior to 1960 (Gutenberg and Richter, 1954). Sequences of large ( $m_{\mathrm{B}}=7.0-7.8$ ) earthquakes occurred mainly in 1905-1907 and 1939-1942. However, seismicity at shallow depth is limited.

Stress axes of intermediate-depth events that occurred after 1960 do not have a preferred orien-

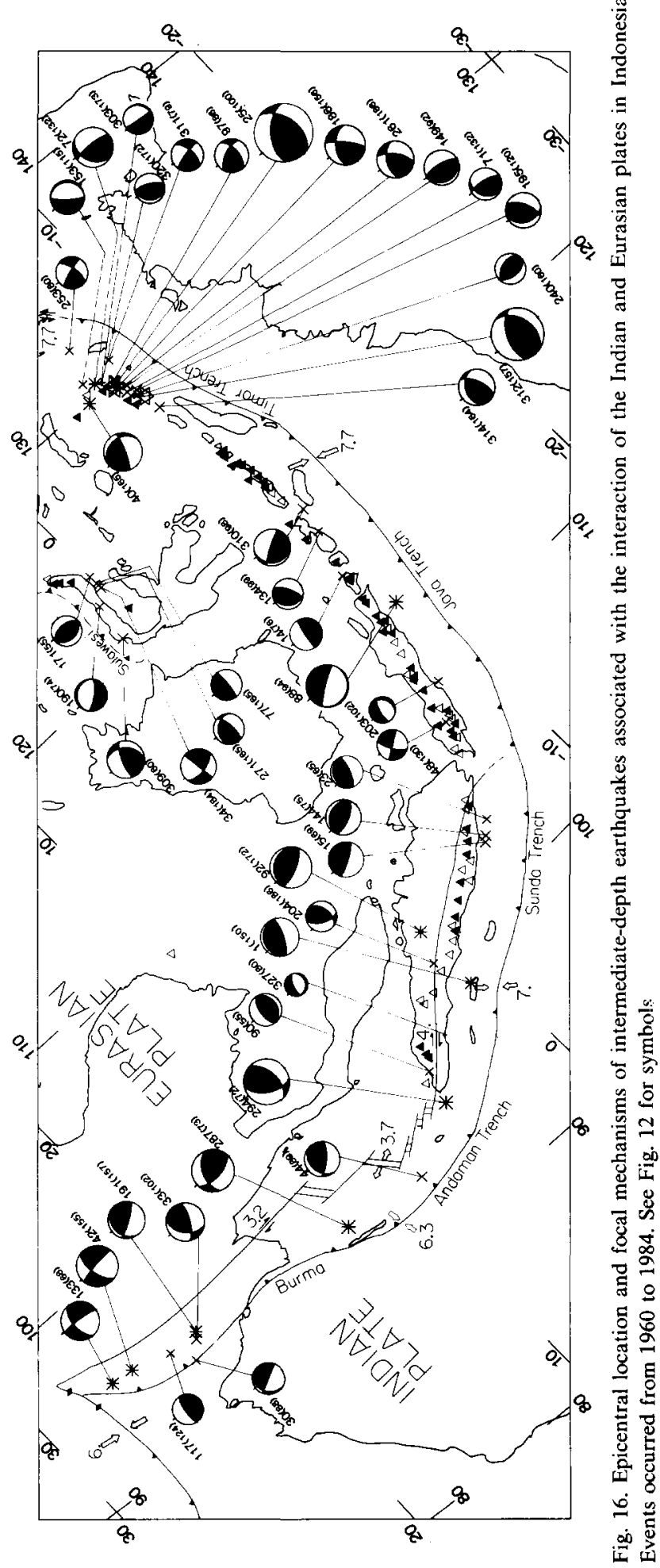


tation (see Fig. 10). Event 171 has an in-plate horizontal axis, in contrast to events 309, 34, 271 and 77 for which the compression axes are normal to the slab. Events 190 and 309 are down-dip tensional events. Interpretation of events in this region is difficult since the geometry of the downgoing plate as well as the tectonics of the region are poorly understood.

\subsection{Indonesia}

The Indo-Australian plate subducts and translates along the Sunda arc from Burma to the Banda Sea (Fig. 16). Various segments are defined by differences in the subducting lithosphere age, the convergence direction between adjacent plates, the maximum seismicity depth (see Tables I and II), and the strength of coupling at the interplate boundary.

\subsubsection{Burma-Andaman Sea}

Oblique convergence between India and Eurasia occurs in the Burma region, where strong coupling is reflected by several large shallow events that occurred in $1897\left(M_{\mathrm{S}}=8.5\right), 1912\left(M_{\mathrm{S}}=8.0\right), 1946$ $\left(M_{\mathrm{S}}=7.8\right)$ and $1950\left(M_{\mathrm{S}}=8.5\right)$. The largest intermediate-depth event in the region occurred in $1954\left(m_{\mathrm{B}}=7.4\right.$, Table A). Most intermediate-depth earthquakes have oblique normal-fault mechanisms (down-dip tensional), consistent with detachment of the denser oceanic lithosphere from the more buoyant continental plate. Event 117 indicates tearing of the subducting plate and may be related to some pre-existing structure.

In the Andaman Sea, oblique subduction of the 55-Ma-old seafloor is accompanied by complex back-arc spreading in this region (Eguchi et al., 1979; Banghar, 1987). Seismicity extends to $\sim 100$ $\mathrm{km}$ depth, with most intermediate-depth events $(287,44,294)$ being down-dip tensional. This is in agreement with the model for moderately coupled subduction zones in which down-dip tensional stresses are induced at intermediate depth by the negative buoyancy of the downgoing plate.

\subsubsection{Sunda}

Convergence of the subducting Indian plate to the northeast along the Sunda trench takes place at $\sim 7 \mathrm{~cm} \mathrm{a}^{-1}$. The volcanic chain parallels the trench and seismicity occurs as deep as $300 \mathrm{~km}$. Historic large shallow earthquakes $\left(M_{\mathrm{W}} \approx 8.7\right)$ have occurred along the Sunda trench (Newcomb and McCann, 1987), suggesting at least a moderately coupled interplate boundary. All intermediate-depth events, from event 327 to the north to event 23 to the south, are down-dip tensional events, and are consistent with the model shown in Fig. 8 for intermediate-depth events that occur down-dip of a partially coupled interplate boundary. Compressional axes for most events are normal to the subducting plate; the exception is event 204, which has horizontal in-plate compression. Burbach and Frohlich (1986) predicted lateral compression stresses in the region where this event occurs.

\subsubsection{Java}

South of the Sunda Strait the maximum depth of seismicity increases abruptly to $650 \mathrm{~km}$ depth. The subducting seafloor is 135-Ma-old and the active volcanic chain parallels the Java trench (Fig. 16). No large historic shallow earthquakes are known in this region (McCann et al., 1979; Newcomb and McCann, 1987), indicating that subduction is mostly aseismic in this $1700-\mathrm{km}$-long trench. In addition, the 1977 Sumbawa normalfault earthquake ( $M_{\mathrm{W}}=8.3$ ), which occurred under the trench axis in response to bending of the lithosphere induced by the slab pull (Spence, 1986), is also consistent with an uncoupled interplate boundary (see Fig. 8). Several large $\left(m_{\mathrm{B}} \geq 7\right)$ intermediate-depth events occurred prior to 1950 in this region (Table $\mathrm{A}$ ).

Event 48 has an in-plate tension axis that could be induced by the lateral bending of the Indian plate as it subducts in this region (Burbach and Frohlich, 1986). Events 88 and 134 are down-dip tensional, whereas the shallower event, 14, is down-dip compressional. Note the relative location of this event with respect to the trench axis and the stress orientation of these events suggest the possible presence of a double seismic zone. Event 310 may be a tear fault.

\subsubsection{Timor}

In the Banda Sea, the subducting lithosphere bends sharply along the Timor trench, where the 
intermediate and deep seismicity define a highly contorted plate (Cardwell and Isacks, 1978). The complexity of the tectonics of the surrounding region contributes to the different interpretations of the shape of the downgoing plate under the Banda Sea (e.g., Burbach and Frohlich, 1986). During this century, many large intraplate earthquakes (Table $\mathrm{A}$ ) have occurred in this region, but only a few large shallow events have been detected.

All intermediate-depth events in the Banda Sea, from event 314 , to the easternmost event 253 , have nearly down-dip tensional focal mechanisms induced by the downgoing plate. Note that the compressional axes of the Banda Sea events in Fig. 16, rotate from a nearly $\mathrm{N}-\mathrm{S}$ direction to a more easterly direction as the subducted plate bends northward (Cardwell and Isacks, 1978; Welc and Lay, 1987). The compression axes of these events rotate systematically around the bend in the downgoing lithosphere. The largest events which occurred recently in the Banda Sea are the $1963\left(25, m_{\mathrm{B}}=7.8\right)$ and the $1983\left(312, M_{\mathrm{W}}=7.4\right)$ earthquakes. These events are studied in detail by Osada and Abe (1981), Welc and Lay (1987) and Michael-Leiba (1984). The 1963 event may involve detachment of the oceanic lithosphere at the leading edge of the Australian continental shelf.

\subsection{Southwest Pacific}

The southwest Pacific region is characterized by subduction along deep trenches which parallel a system of island arcs and marginal basins from New Guinea to New Zealand (Fig. 17). The interaction between the relatively old seafloor of the

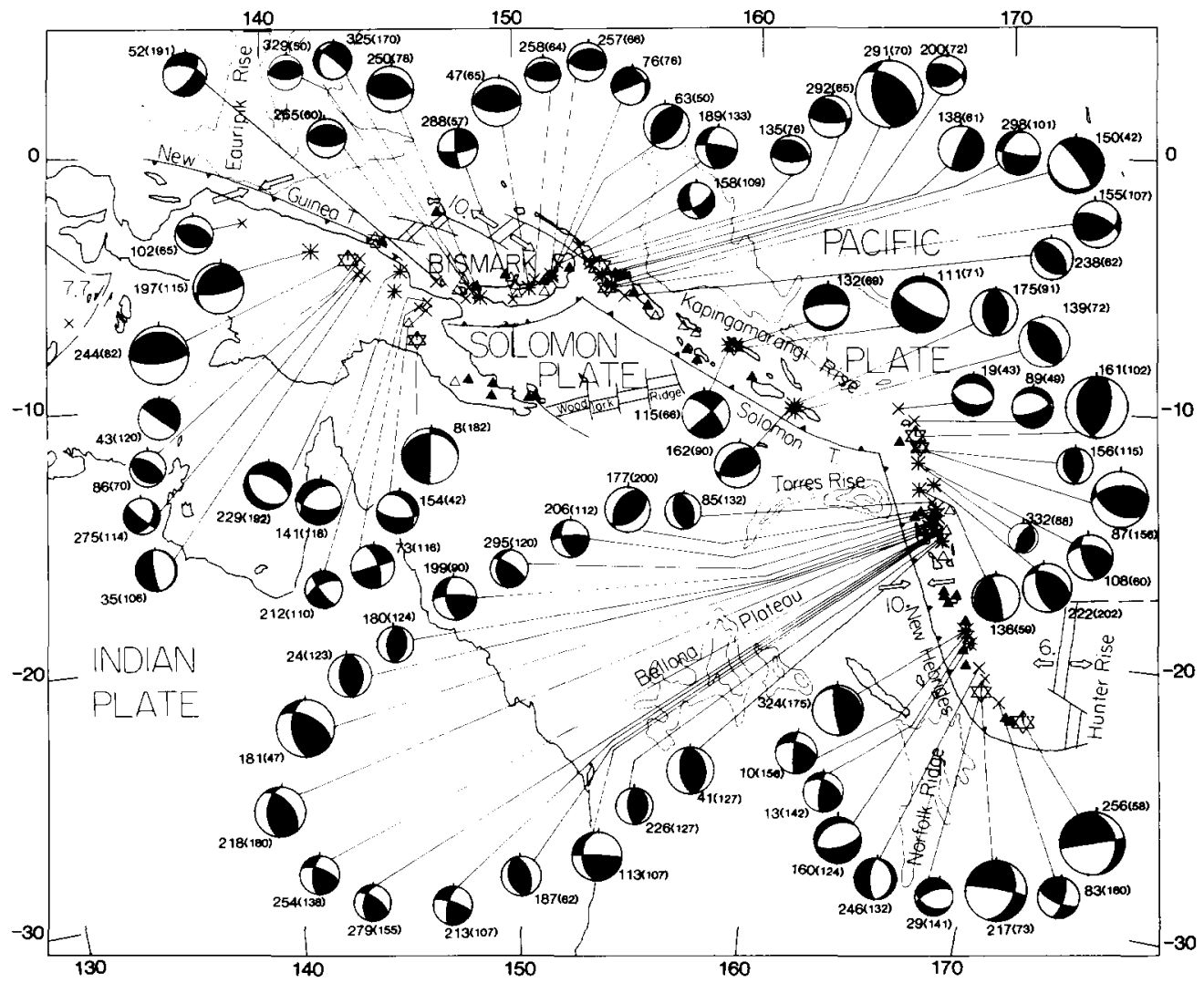

Fig. 17. Intermediate-depth earthquakes $(M \geq 6.0)$ in the New Britain, Solomon, and New Hebrides regions, where the Indian and Solomon plates interact with the Pacific plate. Events occurred from 1960 to 1984 . See Fig. 12 for symbols. 
Pacific and Indo-Australian plates and the younger Solomon and Bismark plates defines different subduction zones in this complex tectonic region. The rupture lengths of most large shallow earthquakes in the southwest Pacific region are $<150 \mathrm{~km}$ long (McCann et al., 1979), indicating moderate coupling in the region. Christensen and Ruff (1987) observed that in this region both compressional and tensional events occur in the outer-rise region. This contrasts with the uncoupled subduction zones along Indonesia and around the Philippine Sea in which only tensional outer-rise events occur.

\subsubsection{New Guinea}

Subduction along the northeast coast of New Guinea defines a downgoing plate to $\sim 500 \mathrm{~km}$ depth along the western end of the New Guinea trench (Pascal, 1979). During this century several large $\left(M_{\mathrm{S}}>7.5\right)$ shallow events have occurred along the New Guinea trench but had very small rupture zones (McCann et al., 1979). A few intermediate-depth events with $m_{\mathrm{b}} \leq 7.2$ have occurred in this region.

Since the shape of the subducting lithosphere under New Guinea is poorly understood and the stress axis distribution of intermediate-depth events in this region does not show a consistent pattern, it is difficult to interpret the intraplate seismicity in this area. Nevertheless, we may emphasize that focal mechanisms of intermediatedepth earthquakes vary rapidly westward along the region from event 102 to event 73 , and that the largest events $(8,244)$ with $M \approx 7.3$ may indicate hinge faulting. Mislocation of intermediate-depth events that occur between the New Guinea and New Britain subduction zones may also confuse the interpretation of the tectonics and the seismic history of this region.

\subsubsection{New Britain-New Ireland-Solomon}

The Solomon plate subducts northward with a steep dip angle to depths of $-500 \mathrm{~km}$ along the New Britain trench. The chain of volcanoes parallels the trench (Fig. 17) along New Britain and the Solomon Islands. The Solomon plate bends abruptly under the New Ireland region and subducts in a northeasterly direction under the Pacific plate (Johnson and Molnar, 1972; Pascal, 1979;
Burbach and Frohlich, 1986). Large shallow earthquakes in this region occur as multiplet events, with the most recent sequences occurring after 1966 (Lay and Kanamori, 1980; Wesnousky et al., 1986). The occurrence of large shallow events located close in time and space indicates that this region has moderate coupling at the interplate boundary.

Most intermediate-depth events in the New Britain region have nearly vertical tension axes and compression axes normal to the plate, consistent with the orientation of stresses induced by the negative buoyancy of the downgoing plate. Exceptions are events 76 and 288, which indicate in-plate deformation. Events 52 and 158, which are deeper and near the edges of the New Britain subduction zone, have in-plate compressional axes. Christensen and Ruff (1987) reported compressional outer-rise events offshore from the western segment of the New Britain trench, on the basis of which they infer that the interplate boundary is under compression. Large thrust events occurred there in 1945 and 1946. Note that event 47, with focal depth of $65 \mathrm{~km}$ had many aftershocks within a week. If this event was actually shallower, its focal mechanism would also be consistent with thrusting on the interplate boundary.

The New Ireland region includes those events located near the corner joining the New Britain and Solomon trenches. This small region, between $152.5^{\circ}$ and $155^{\circ} \mathrm{E}$ longitude and $2^{\circ}$ and $7^{\circ} \mathrm{S}$ latitude, has many large intermediate-depth events during this century (Table A). The interplate boundary breaks in doublet events (Lay and Kanamori, 1980) that occur about every 30 years, the most recent of which occurred on July 14 $\left(M_{\mathrm{W}}=8.0\right)$ and July 26, $1971\left(M_{\mathrm{W}}=8.1\right)$. Most events at intermediate depth in the New Ireland region have down-dip tensional mechanisms consistent with stresses induced by the pull of the slab. Event 138 indicates hinge faulting within the plate. However, event 298 has a down-dip compressional mechanism. This event occurred downdip, and after, the July 14, 1971 thrust earthquake, and is consistent with the dynamic model shown in Fig. 3b. Although the down-dip tensional event $150\left(m_{\mathrm{b}}=7.1\right)$ that occurred on July 19,1971 is located down-dip of the July 14 event, it may have 
helped trigger the July 26 thrust earthquake that broke the adjacent segment near the corner of the New Britain trench (see fig. 3 in Lay and Kanamori, 1980). Christensen and Ruff (1987) reported tensional outer-rise events after the occurrence of the 1971 New Ireland doublet. The New Britain-New Ireland region, albeit complicated, agrees with the simple model of partially coupled regions shown in Fig. 8, in which vertical tensional stresses are induced at intermediate depth from the slab's weight. Also, temporal changes of focal mechanisms are observed in the outer-rise and intermediate-depth regions associated with the occurrence of large, underthrusting earthquakes.

The Solomon trench is the boundary between the Pacific plate and the Solomon and IndoAustralia plates. The Woodlark Ridge subducts along the central part of the Solomon trench, where few large subduction events occur (McCann et al., 1979). However to the north and south of this topographic high, the interplate boundary has been very active. It is characterized by the occurrence of multiplet events with rupture lengths of $\sim 100 \mathrm{~km}$ (Lay and Kanamori, 1980; Wesnousky et al., 1986). Seismicity extends $200 \mathrm{~km}$ deep in this region. At intermediate depth, events 115, 132 and 111, located near the northern edge and down-dip of the Woodlark ridge, indicate a complex stress state, possibly along the boundary of the Solomon and Indo-Australian plates at depth. Events 175, 139 and 162 have vertical tension axes but variable compression axes induced by slab pull and deformation of the plate. These events occurred before the recent 1978-1979 thrust earthquake sequences in this region (Wesnousky et al., 1986).

\subsubsection{New Hebrides}

The New Hebrides subduction zone dips steeply eastward and delineates the boundary between the Indo-Australian plate and the Pacific plates. The volcanic chain parallels the New Hebrides trench for most of its length (Fig. 17). The interplate boundary has been very active during this century and it is the most active region at intermediate depth (Table A). Seismicity is continuous to a depth of $300 \mathrm{~km}$ in the New Hebrides. (Pascal et al., 1973; Isacks et al., 1979; McCann et al., 1979). Events 19 and 89, located at the corner of the Solomon and New Hebrides trenches, have vertical compressive stresses and horizontal tensional stresses aligned with the plate, which reflect the lateral bending of the downgoing Indo-Australian plate (Burbach and Frohlich, 1986). All other intermediate-depth events in the northern segment of the New Hebrides trench, from event 161 to the north to event 41 , have a down-dip tension axis and a compression axis normal to the plate, consistent with the orientation of stresses induced by the negative buoyancy of the slab. Events 41 and 181 , which may be associated with subduction of the Torres rise, had many aftershocks within a week (see Fig. 9).

Back-arc spreading occurs in the southern segment of the New Hebrides trench. Intermediatedepth events that occur in this region show a complex pattern in the stress distribution (see Fig. $10)$. Events $(324,10,13)$ in this southern segment of the New Hebrides trench have down-dip tensional axes and arc-normal compressional axes, similar to events to the north, as discussed above. Event 160 has a down-dip compression and a horizontal tension axis within the plate. This event is located in the region where Burbach and Frohlich (1986) computed lateral extension to be present. Event 246 shows intraplate deformation. Events 256, 83, 217 and 29, near the southern bend where the boundary involves left-lateral strike-slip motion, are hinge-faulting events.

Large, shallow events occurred in the New Hebrides region in $1966\left(M_{\mathrm{S}}=7.9\right), 1985\left(M_{\mathrm{S}}=\right.$ 7.6, 7.6), $1973\left(M_{\mathrm{S}}=7.9\right), 1965\left(M_{\mathrm{S}}=7.7\right)$, and $1980\left(M_{\mathrm{W}}=7.8\right)$ from north to south along the interplate boundary. However, the relationship between the large intermediate-depth events and the shallow underthrusting events is unclear.

\subsubsection{Tonga-Kermadec-New Zealand}

The Tonga-Kermadec trench system is a fairly linear island arc with seismicity occurring as deep as $650 \mathrm{~km}$ (Fig. 18). The Pacific plate subducts to the west underneath the Indian plate, delineating a smooth slab to $\sim 400 \mathrm{~km}$ depth. However, deep events indicate a highly contorted plate below 400 $\mathrm{km}$ depth (Billington, 1980; Giardini and 


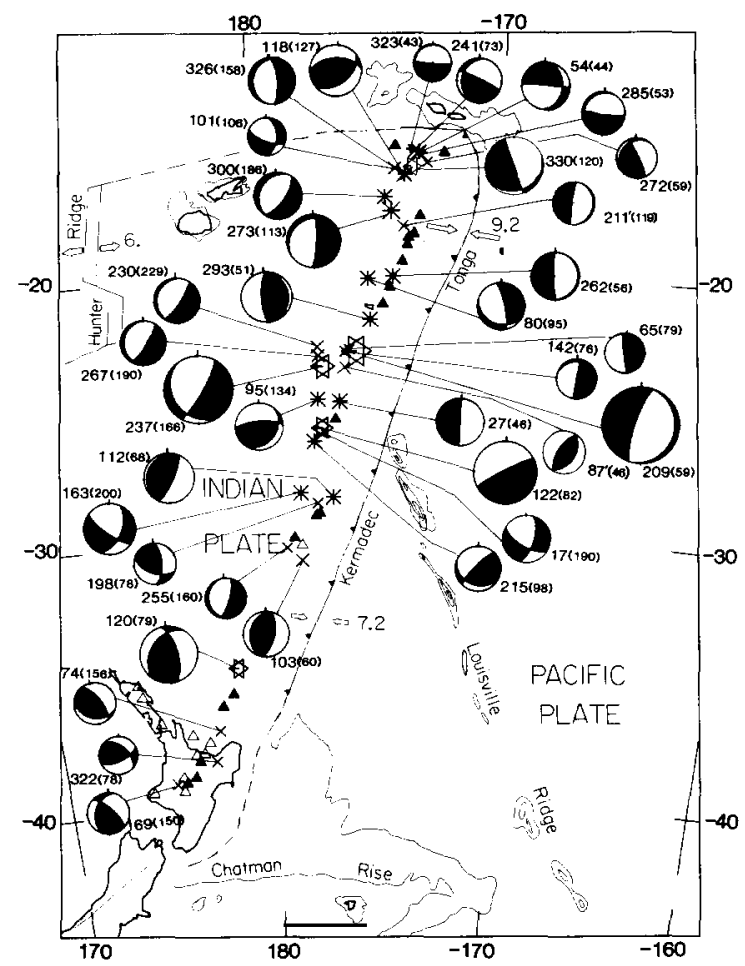

Fig. 18. Focal mechanisms and epicenters of events occurred in the period 1960 to 1984 (with $M \geq 6.0$ ) in the Tonga, Kerma$\mathrm{dec}$ and New Zealand regions, where the Pacific plate underlies the Indian plate. For symbols see Fig. 12.

Woodhouse, 1984). The slab dip is $55^{\circ}$ under Tonga and increases to $70^{\circ}$ farther south under the Kermadec and New Zealand trenches. The volcanic chain, which parallels the Tonga trench, disappears in the region where the Louisville ridge is being subducted (Fig. 18).

Large, shallow events along the northern section of the Tonga trench occurred before 1950 (McCann et al., 1979), although the mechanisms of these events are unknown. No large underthrusting mechanisms are known to have occurred along the Tonga arc north of the Louisville ridge. However, outer-rise compressional events have occurred near $22^{\circ} \mathrm{S}$ (Christensen and Ruff, 1987). Along the northern boundary of the Kermadec trench several thrust events have occurred after 1960 with a large doublet in $1976\left(M_{\mathrm{S}}=7.7,8.0\right)$. A large thrust event occurred in $1982\left(M_{\mathrm{S}}=8.2\right)$ at the location where the Louisville ridge intersects the Tonga trench. The interplate boundary along the Kermadec region is thus partially coupled, as indicated by the maximum rupture lengths of large shallow thrust events of $\sim 150 \mathrm{~km}$ (McCann et al., 1979) while the Tonga region appears to be uncoupled. Most of the deep seismicity occurs in the Tonga trench (Vassiliou et al., 1984; Giardini, 1987), and this region is also very active at intermediate depth.

Intermediate-depth events near the northern boundary of Tonga, where the Pacific plate is strongly contorted, are characterized by in-plate extension, in agreement with Burbach and Frohlich's (1986) calculations for this region. Farther south, most intermediate-depth events have downdip compressional stresses, which are transmitted from the deep seismic region to nearly all depths in the subducting Tonga lithosphere. The few down-dip tensional events in this region are associated with subduction of the Louisville ridge, which appears to increase the coupling of the interplate boundary locally. Events 262 and 27 with $m_{\mathrm{b}}=6.5$ are located down-dip of the Louisville ridge at its inferred northern and southern edges (Giardini and Woodhouse, 1984). The large normal-fault 1977 Tonga earthquake (209) was located down-dip and to the north of the large $1982\left(M_{\mathrm{S}}=7.7\right)$ thrust earthquake. An outer-rise compressional event occurred before 1982 from the thrust rupture zone (Christensen and Ruff, 1987). Following the 1977 event deeper down-dip compressional events occurred $(230,237,267)$. This sequence is consistent with the dynamic model shown in Fig. $3 b$.

Most events in the Kermadec region have a down-dip tensional axis and arc-normal compression. Event 163, the deepest event in Fig. 18, has down-dip compressional stresses similar to deeper events in the region (e.g., Isacks and Molnar, 1969). In 1963 a down-dip tensional event (103, $\left.m_{\mathrm{b}}=6.4\right)$ occurred down-dip of the 1976 aftershock zone. Event $255\left(m_{\mathrm{b}}=6.1\right)$ which occurred down-dip and after the two 1976 events, is downdip compressional. Furthermore, Christensen and Ruff (1987) reported that an outer-rise compressional event occurred prior to the 1976 Kermadec doublet as did a smaller outer-rise tensional event. This sequence is generally consistent with the dynamic model in Fig. 3b. 


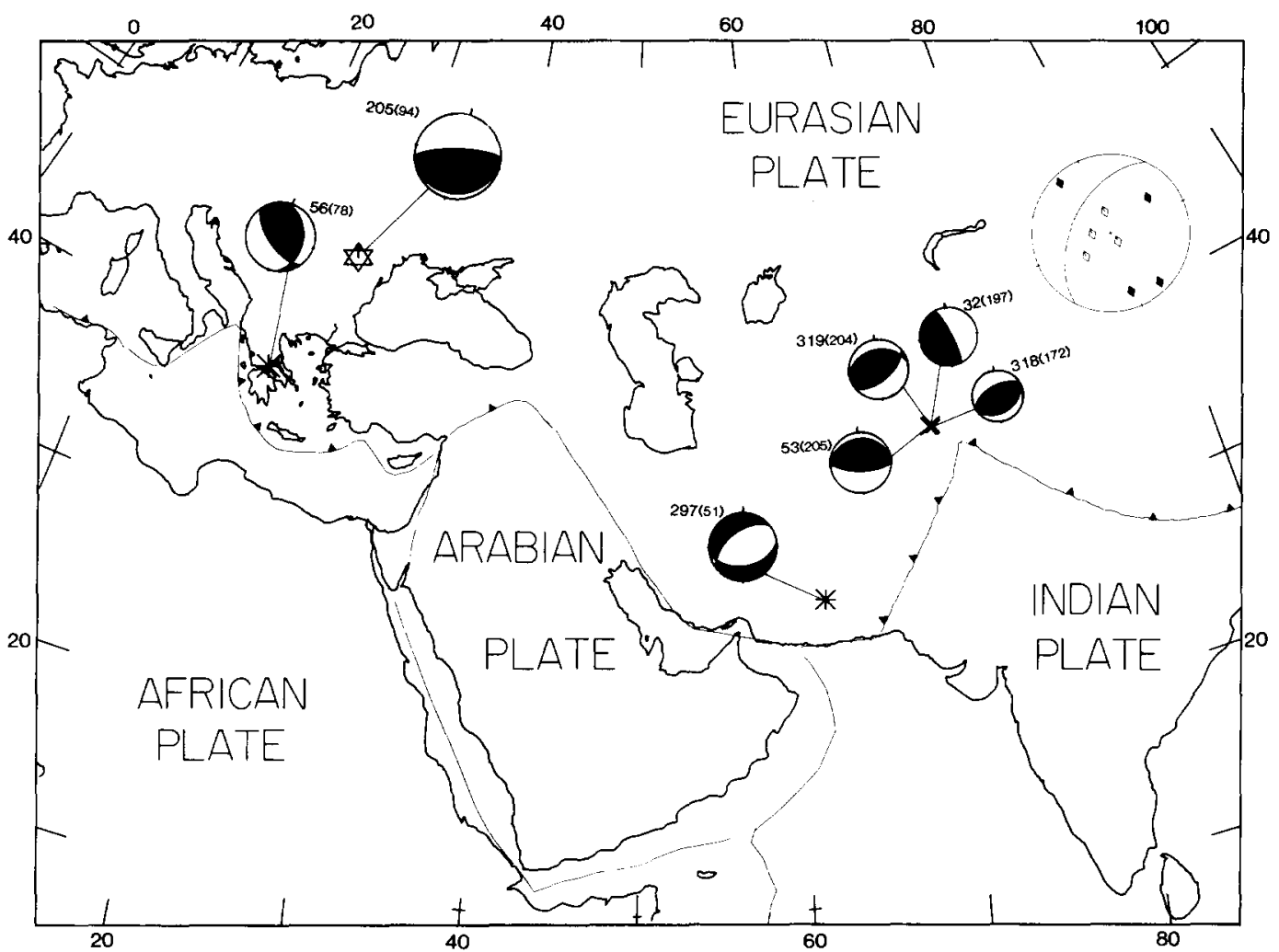

Fig. 19. Events with $M \geq 6.0$ that occurred at intermediate depth between 1960 and 1984 in regions where continental coalitions are taking place. See Fig. 12 for symbols. The $P$ and $T$ axis (closed and open symbols, respectively) of events that occurred in the Indu-Kush and Hellenic arc regions are shown in a lower hemisphere projection. Circles are events shallower than $100 \mathrm{~km}$ and diamonds indicate deeper events.

In New Zealand, the intermediate-depth events $(74,322,169)$ have down-dip tensional axes resulting from the negative buoyancy of the downgoing lithosphere.

\subsection{Alpine-Himalaya Belt}

Figure 19 shows the intermediate-depth events that occurred between 1960 and 1984 in the Alpine-Himalaya belt.

\subsubsection{Hindu-Kush}

Seismicity concentrates at $\sim 220 \mathrm{~km}$ depth in the Hindu-Kush region, where large $m_{\mathrm{B}}>7$ events are frequent (Table $A$ ). Intermediate-depth events have near vertical tensional axes, suggesting that the denser oceanic lithosphere which subducted a few million years ago is now being detached from the more buoyant continental lithosphere.

\subsubsection{Iran}

The seismotectonics of the Iran-Pakistan region have been studied by several investigators (e.g., Jackson and Fitch, 1979, 1981; Jacob and Quittmeyer, 1979). Intermediate-depth events occur along the Makran subduction zone, where the Arabian plate is subducting beneath Eurasia at a shallow dip to $\sim 80 \mathrm{~km}$ depth. Event $297\left(M_{\mathrm{W}}=\right.$ 6.7) has a nearly vertical compression axis and a tension axis nearly horizontal and in the direction of convergence. At greater depth, Jacob and Quittmeyer (1979) reported down-dip tensional events in this area.

\subsubsection{Rumania}

Although the detailed structure of the subducting lithosphere under Rumania is poorly defined, it appears that there is an almost vertical slab that 
strikes NE-SW and dips to the northwest under this region (Isacks and Molnar, 1969). Seismicity concentrates between 100 and $150 \mathrm{~km}$ depth. Two large events occurred in this region recently, in $1940\left(m_{\mathrm{B}}=7.3\right)$ and in $1977\left(205, M_{\mathrm{W}}=7.4\right)$. The latter event indicates near vertical tension stresses similarly to events in the Hindu-Kush region.

\subsubsection{Greece}

The Mediterranean seafloor is subducting eastward along the Hellenic arc under Eurasia at an angle of $60^{\circ}$ to $\sim 200 \mathrm{~km}$ depth (McKenzie, $1978)$. Event $56\left(m_{\mathrm{b}}=6.7\right)$ has down-dip tensional stresses probably induced by the negative buoyancy of the subducting plate. A larger earthquake, $m_{\mathrm{B}}=7.7$, occurred in Greece in 1926 at $100 \mathrm{~km}$ depth (Table A).

\section{Discussion and conclusions}

In this study we have given an overview of the global occurrence of large intermediate-depth earthquakes. By considering only the largest events, we have restricted our attention to the gross spatial and temporal characteristics of the deformation occurring in each region. A fundamental assumption in the study of intermediatedepth events is that the earthquakes serve as stress gauges for the intraplate environment, reflecting static and dynamic stress fields in a competent, quasi-elastic subducting slab. Many interesting aspects of intermediate-depth earthquake phenomena, such as double Benioff zones, plate contortions, and shear zone lineaments are only revealed by considering all earthquakes in each region. Our purpose is to investigate the spatial and temporal relationship between the intermediatedepth activity and interplate events by using two relatively complete catalogs of large intermediatedepth events rather than to unveil all of the complexity of each subducting slab.

The first catalog we considered is the historic record of large $\left(m_{\mathrm{B}}>7\right)$ events that have occurred within subducting slabs in this century. This catalog gives earthquake locations only, for few of the focal mechanisms are known. Only for events in the last 25 years are the depths considered relia- ble. According to this catalog the New Hebrides region is the most active region at intermediate depths, while Tonga is the most active below 400 $\mathrm{km}$ depth. Other regions with a large number of intermediate-depth events are the Altiplano, Timor, Sulawesi, Scotia and New Ireland, all of which have strong contortions of the subducted lithosphere. Other active regions include North Chile, Kuriles, Ryukyus and Philippines. Time sequences of the occurrence of large interplate subduction zone events for all regions do not reveal any simple pattern of stress migration down-dip; however, there is a marked tendency for sequences of large events within the plate to occur at periods when no large interplate activity is taking place. Several regions exhibit long time scale temporal variations in intraplate activity, such as Ryukyu, Sulawesi, Peru, Philippines and New Guinea.

We tested the dependence of the maximum size intermediate and deep focus earthquakes on various parameters of the subduction zones. Convergence rate appears to influence the maximum size of deep earthquakes, while the angle of descent of the subducted slab is inversely proportional to maximum event size. No dependence on age of the slab or maximum depth of penetration was detected.

In order to examine the spatial and temporal variations in stress orientation of large intermediate-depth earthquakes, a second catalog is compiled. This catalog comprises focal mechanisms of all intermediate depth $(40-200 \mathrm{~km})$ events with $M \geq 6.5$ for the period 1960-1984. We determined 40 new focal mechanisms and confirmed the focal depth and focal parameters for many additional events. A catalog with 335 events, which includes published focal mechanisms of the events with $M>6$ in the same depth range and time period.

We first considered the general spatial characteristics of the focal mechanisms of the larger events $(M>6.8)$ in this catalog. Four categories were defined: (1) normal-fault events $(44 \%)$, and (2) reverse-fault events $(33 \%)$, both with a strike nearly parallel to the trench axis; (3) normal or reverse-fault events with a strike significantly oblique to the trench axis (10\%) and (4) tear faulting 
events (13\%). The focal mechanisms of type 1 events occur at the base of strongly or moderately coupled subduction zones; similar type events occur near the trench axis in uncoupled zones. Type 2 events have near vertical tension axes and occur mainly in regions that have partially coupled or uncoupled subduction zones, and where the observed continuous seismicity extends deeper than $300 \mathrm{~km}$. We advanced a simple model, in which the increased dip of the downgoing slab associated with weakly coupled subduction zones induces nearly vertical tensional stress at intermediate depth and, consequently, the change in focal mechanism from type 1 to type 2 events. Events of type 3 occur where the trench axis bends sharply, causing horizontal (parallel to the trench strike) extensional or compressional intraplate stress. Type 4 are hinge-faulting events associated with lateral segmentation of the subducting slab.

We determined the number of aftershocks that occurred within one week of the mainshock reported by the NOAA and ISC catalogs for the intermediate-depth events with $M \geq 6.5$ that occurred between 1960 and 1984. About $48 \%$ of the events had no aftershocks, $37 \%$ of the events had between one and five aftershocks and only $15 \%$ more than five aftershocks. There is a slight correlation between mainshock magnitude and a number of aftershocks. However, all events with more than ten aftershocks are located in the region associated with bends in the subducted slab.

Detailed regional observations, on the basis of all the events $(M>6)$ in our focal mechanism catalog, support the idea that the subducting lithosphere acts as a stress guide in response to spatial or temporal changes of the strength of interplate coupling.

Intermediate-depth earthquakes for uncoupled, or partially coupled, regions involve predominantly reverse faulting, with nearly vertical tension axes. Regions that are partially or weakly coupled are the Lesser and Greater Antilles, the Scotia arc, Ryukyu, Philippines, Timor, New Hebrides, and Solomon subduction zones. Uncoupled regions include central Japan, Izu-Bonin, Mariana and Java. The events occur in response to the negative buoyancy of the subducted slab. These regions have relatively long and steeply dipping seismic zones and may exhibit double seismic zones in response to other factors such as unbending of the downgoing lithosphere. In the uncoupled regions, no temporal changes in the focal mechanisms of either outer-rise or intermediatedepth earthquakes are observed while such temporal behavior is sometimes observed in the partially coupled regions, as is the case for the Solomon Islands.

Intermediate-depth events down-dip of moderately or strongly coupled regions have normal faulting mechanisms near the base of the interplate boundary in response to the down-dip pull of the subducted lithosphere. In contrast, regions that are uncoupled produce large normal faulting events near the trench axis, where stresses due to bending of the lithosphere are largest. Temporal changes either in the focal mechanisms of intermediate-depth events, from down-dip tensional to down-dip compressional events, or a reduction in the seismic activity at intermediate depth are frequently observed after the occurrence of large thrust earthquakes along strongly coupled regions.

Along Middle and South America, where the interplate boundary varies from moderate to strongly coupled, intermediate-depth earthquakes are generally normal-fault events that occur before and down-dip of future large subduction earthquakes. In most regions along Middle and South America, we observe a reduction only in the number of large intermediate-depth events after large subduction events occur in the region. However, down-dip compressional events have been observed after the 1960 Chile and the 1974 Peruvian thrust earthquakes. After the 1964 Alaska earthquake only one large event, a tear fault, has occurred at intermediate depth. Down-dip of the large 1957 Aleutian earthquake a few events indicate coupling at the interplate boundary. This region was very active at intermediate-depth before 1957 when the interplate boundary broke. In addition, one of these events occurred before and down-dip of the large 1986 Andreanof earthquake.

Down-dip of the 1952 and 1959 Kamchatka earthquakes aftershock zones, a down-dip compressional event occurred in 1960. After 1969 only events with down-dip tensional axes have occurred, which indicates that this region is again 
moderately coupled. Large intermediate-depth events under Japan and the South Kurile trench reflect the complexity of the subducting lithosphere in this region, which has been very active throughout this century.

Along the Tonga trench mostly down-dip compressional events are observed; however, the large 1977 event located down-dip of the Louisville ridge had a down-dip tensional mechanism and preceded the large 1982 Tonga thrust event. Temporal changes in the focal mechanisms are also observed with the occurrence of the 1976 Kermadec doublet events.

In conclusion, this overview has confirmed the general complexity of the spatio-temporal occurrence of intermediate-depth earthquakes, with slab pull forces and lateral slab deformation playing the principal role in causing the earthquake occurrence. However, we have presented numerous instances in which temporal variations in the level of activity or stress orientation in the interplate environment are associated with large interplate thrust events. Given that such variations are observed for the very largest events, future investigations of all intraplate activity within the subducted slabs promise to reveal additional features of the seismic cycle.

\section{Acknowledgements}

We thank Bob F. Svendsen for his computer expertise. This research was supported by U.S. Geological Survey Grants 14-08-0001-G1170 and 14-08-002-G1277 (H.K.) and NSF Grant EAR8451715 (T.L.) and a Shell Faculty Career Initiation Grant to T.L. Contribution No. 4504, Division of Geological and Planetary Sciences, California Institute of Technology, Pasadena, California.

\section{Appendix 1}

The depth and time distribution of large intermediate and deep focus earthquakes that occurred between 1904 and 1984 is shown by a series of figures in this appendix. These events are listed by regions in Table A. Earthquake hypocenters were taken from Abe (1981) for most events prior to 1960. A few events were taken from Abe (1984, 1985). The parameters for intermediate-depth events (40-200 km deep) are taken from Table V, while data for deeper events are taken from the NOAA catalog. Magnitudes are $m_{\mathrm{B}}$ for events that occurred before 1975. $M_{\mathrm{W}}$ is given for events that occurred thereafter, and $m_{\mathrm{B}}$ values are calculated from the relation $m_{\mathrm{B}}=0.63 M_{\mathrm{W}}+2.5$ (Kanamori, 1983). In the figures we show the $m_{\mathrm{B}}$ value for all events. The seismic moment, $M_{0}$, can be computed from the relation $\log M_{0}=2.4 m_{\mathrm{B}}+$ 10.1 given by Kanamori (1983) for intermediate and deep focus events.

Figure A1 shows magnitude-depth distribution on the left-hand side. Diamonds indicate events that occurred after 1960 , presumably with more reliable locations. The arrows indicate the extent of continuous seismicity for each region (Table I). Observations made of the variation of magnitude of this century, large, intraplate earthquakes and depth are strongly dependent not only on the accuracy of $m_{\mathrm{B}}$ to measure earthquake size, but also on the accuracy of the event location. We assume that they are approximately correct. Although some regions in South America (Colombia (1), Ecuador (2), Peru (3) and North Chile (5)) have deep events, it is unclear if a continuous slab is present or if the shallow and deep seismicity is unrelated (Stauder, 1975). The seismic activity stops at $\sim 250 \mathrm{~km}$ and reappears at $\sim 550 \mathrm{~km}$ depth at about the same horizontal distance from the trench. Note that the Rivera and the Nankai Trough regions are not included in Table A. These regions have young, short subducting slabs (Table I) and no large event deeper than $40 \mathrm{~km}$ has occurred there during this century.

The figure on the right-hand side displays the time-distance distribution for regions 1-38. Distance along strike for each subduction zone is given in $\mathrm{km}$, the approximate direction of the projection is also indicated. Asterisks show the location of large shallow earthquakes with $M_{\mathrm{S}} \geq$ 7.5 and the approximate extent of the aftershock area for these events is indicated with a horizontal line. Cross-diamonds indicate the location of 
TABLE A

Large intermediate and deep focus events

\begin{tabular}{|c|c|c|c|c|c|c|c|c|c|c|c|}
\hline \multicolumn{3}{|c|}{ DATE } & \multicolumn{2}{|c|}{ TIME } & \multirow{2}{*}{\multicolumn{2}{|c|}{$\begin{array}{l}\text { LATITUDE } \\
\left(+^{\circ} N_{-}{ }^{\circ} S\right) \\
\end{array}$}} & \multirow{2}{*}{\multicolumn{2}{|c|}{$\begin{array}{l}\text { LONGITUDE } \\
\left(+^{\circ} E--^{\circ} W\right)\end{array}$}} & \multirow{2}{*}{$\begin{array}{c}\text { DEPTH } \\
(\mathrm{km}) \\
\end{array}$} & \multicolumn{2}{|c|}{ MAGNITUDE } \\
\hline Year & Mo & Dy & $\mathrm{HrMn}$ & Sec & & & & & & $m_{B}$ & $M_{v}$ \\
\hline 6 & $\mathrm{COI}$ & $\mathrm{OMI}$ & LA (1) & & & & & & & & \\
\hline 1911 & 4 & 10 & 1842 & 24.00 & 9 & 0.00 & -74 & 0.00 & 100.0 & 7.00 & \\
\hline 1911 & 4 & 28 & 0952 & 54.00 & 0 & 0.00 & -71 & 0.00 & 600.0 & 6.90 & \\
\hline 1914 & 5 & 28 & 323 & 54.00 & 9 & 0.00 & -78 & 0.00 & 70.0 & 6.90 & \\
\hline 1925 & 3 & 29 & 2112 & 37.00 & 8 & 0.00 & -78 & 0.00 & 60.0 & 7.10 & \\
\hline 1970 & 7 & 31 & 1708 & 5.40 & -1 & 27.54 & -72 & 33.48 & 651.0 & 7.50 & \\
\hline 1979 & 11 & 23 & 2340 & 29.80 & 4 & 48.30 & -76 & 13.02 & 1086 & 7.00 & 7.20 \\
\hline 6 & ECT & $\mathrm{ADC}$ & $R(2)$ & & & & & & & & \\
\hline 1906 & 9 & 28 & 1524 & 54.00 & -2 & 0.00 & -79 & 0.00 & 150.0 & 7.50 & \\
\hline 1921 & 12 & 18 & 1529 & 35.00 & -2 & 30.00 & -71 & 0.00 & 650.0 & 7.50 & \\
\hline 1922 & 1 & 17 & 350 & 33.00 & -2 & 30.00 & -71 & 0.00 & 650.0 & 7.40 & \\
\hline 1937 & 7 & 19 & 1935 & 24.00 & -1 & 30.00 & -76 & 30.00 & 190.0 & 7.10 & \\
\hline 1971 & 7 & 27 & 202 & 4960 & -2 & 44.88 & -77 & 25.74 & 135.0 & 7.30 & \\
\hline 1983 & 4 & 12 & 1207 & 5460 & -4 & 53.40 & -78 & 10.80 & 111.1 & 6.90 & 6.97 \\
\hline 13 & PEF & $U(3)$ & & & & & & & & & \\
\hline 1915 & 4 & 23 & 1529 & 1800 & -8 & 0.00 & -68 & 0.00 & 650.0 & 6.90 & \\
\hline 1941 & 9 & 18 & 1314 & 9.00 & -13 & 45.00 & -72 & 15.00 & 100.0 & 7.00 & \\
\hline 1950 & 7 & 9 & 440 & $4 \infty$ & -8 & 0.00 & -70 & 45.00 & 650.0 & 7.00 & \\
\hline 1950 & 7 & 9 & 450 & 500 & -8 & 0.00 & -70 & 45.00 & 6500 & 7.00 & \\
\hline 1950 & 12 & 10 & 250 & 42.00 & -14 & 15.00 & -75 & 45.00 & 80.0 & 7.10 & \\
\hline 1959 & 7 & 19 & 1506 & 10.00 & -15 & 0.00 & -70 & 30.00 & 200.0 & 7.10 & \\
\hline 1958 & 7 & 26 & 1737 & 9.00 & -13 & 30.00 & -69 & 0.00 & 620.0 & 7.20 & \\
\hline 1961 & 8 & 31 & 148 & 37.50 & -10 & 42.00 & -70 & 54.00 & 6260 & 7.00 & \\
\hline 1961 & 8 & 31 & 157 & 8.00 & -10 & 30.00 & -70 & 42.00 & 629.0 & 7.30 & \\
\hline 1961 & 8 & 19 & 509 & 49.50 & -10 & 48.00 & -71 & 0.00 & 649.0 & 7.20 & \\
\hline 1963 & 8 & 15 & 1725 & 6.00 & -13 & 48.00 & -69 & 15.00 & 543.0 & 7.30 & \\
\hline 1963 & 11 & 9 & 2115 & 30.00 & -8 & 48.00 & -71 & 42.00 & 576.0 & 7.20 & \\
\hline 1970 & 5 & 31 & 2023 & 27.30 & -9 & 10.56 & -78 & 49.38 & 48.0 & 7.80 & \\
\hline 26 & AL? & PL & NO (4) & & & & & & & & \\
\hline 1910 & 10 & 4 & 2300 & 6.00 & -22 & 0.00 & -69 & 0.00 & 120.0 & 7.20 & \\
\hline 1913 & 5 & 8 & 1835 & 24.00 & -17 & 0.00 & .74 & 30.00 & 200.0 & 6.90 & \\
\hline 1914 & 2 & 26 & 458 & 1200 & -18 & 0.00 & -67 & 0.00 & 130.0 & 7.20 & \\
\hline 1915 & 6 & 6 & 2129 & 37.00 & -18 & 30.00 & -68 & 30.00 & 160.0 & 7.30 & \\
\hline 1916 & 8 & 25 & 944 & 42.00 & -21 & 0.00 & -68 & 0.00 & 180.0 & 7.00 & \\
\hline 1922 & 3 & 28 & 357 & 54.00 & -21 & 0.00 & -68 & 0.00 & 90.0 & 7.10 & \\
\hline 1922 & 10 & 11 & 1449 & 50.00 & -16 & 0.00 & -72 & 30.00 & 50.0 & 7.60 & \\
\hline 1929 & 10 & 19 & 1012 & 52.00 & -23 & 0.00 & -69 & 0.00 & 100.0 & 7.10 & \\
\hline 1933 & 10 & 25 & 2328 & 16.00 & -23 & 0.00 & -66 & 42.00 & 220.0 & 7.00 & \\
\hline 1940 & 10 & 4 & 754 & 42.00 & -22 & 0.00 & -71 & 0.00 & 75.0 & 7.10 & \\
\hline 1940 & 12 & 22 & 1859 & 46.00 & -15 & 30.00 & -68 & 30.00 & 230.0 & 6.90 & \\
\hline 1941 & 4 & 3 & 1521 & 39.00 & -22 & 30.00 & -66 & 0.00 & 260.0 & 7.20 & \\
\hline 1943 & 3 & 14 & 1837 & 56.00 & -20 & 0.00 & -69 & 30.00 & 150.0 & 7.10 & \\
\hline 1943 & 12 & 1 & 1034 & 46.00 & -19 & 30.00 & .69 & 45.00 & 80.0 & 7.10 & \\
\hline 1948 & 5 & 11 & 855 & 41.00 & -17 & 30.00 & -70 & 15.00 & 70.0 & 7.40 & \\
\hline 1949 & 4 & 25 & 1354 & 59.00 & -19 & 45.00 & -69 & 0.00 & 110.0 & 7.20 & \\
\hline 1952 & 9 & 21 & 230 & 35.00 & -21 & 45.00 & -65 & 45.00 & 260.0 & 7.10 & \\
\hline 1953 & 12 & 7 & 205 & 24.00 & -22 & 6.00 & -68 & 42.00 & 128.0 & 7.20 & \\
\hline 1959 & 7 & 19 & 1506 & 10.00 & -15 & 0.00 & -70 & 30.00 & 200.0 & 7.10 & \\
\hline 1957 & 11 & 29 & 2219 & 38.00 & -21 & 0.00 & -66 & 0.00 & 200.0 & 7.40 & \\
\hline 1958 & 1 & 15 & 1914 & 29.00 & -16 & 30.00 & -72 & 0.00 & 60.0 & 7.00 & \\
\hline 1959 & 6 & 14 & 12 & 2.00 & -20 & 25.20 & -69 & 0.00 & 83.0 & 7.50 & \\
\hline 1960 & 1 & 13 & 1540 & 3400 & -16 & 0.00 & -72 & 0.00 & 200.0 & 7.50 & \\
\hline 1967 & 12 & 27 & 917 & 55.70 & -21 & 12.00 & -68 & 18.00 & 1350 & 7.00 & \\
\hline 1974 & 1 & 2 & 1042 & 29.90 & -22 & 32.28 & -68 & 23.82 & 105.0 & 7.10 & \\
\hline 1976 & 11 & 30 & 40 & 5780 & -20 & 31.20 & -68 & 55.14 & 820 & 7.30 & \\
\hline
\end{tabular}




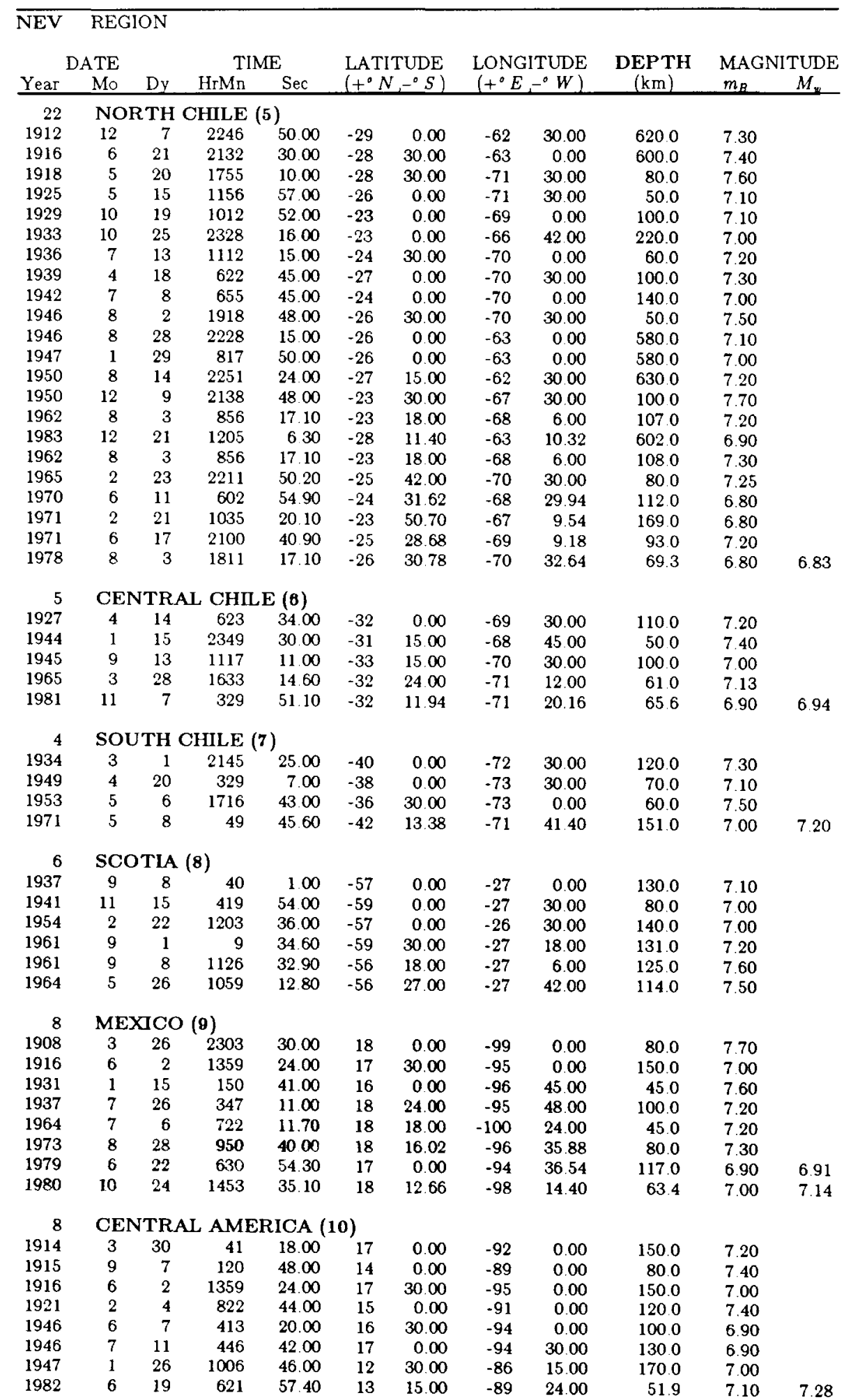


TABLE A (continued).

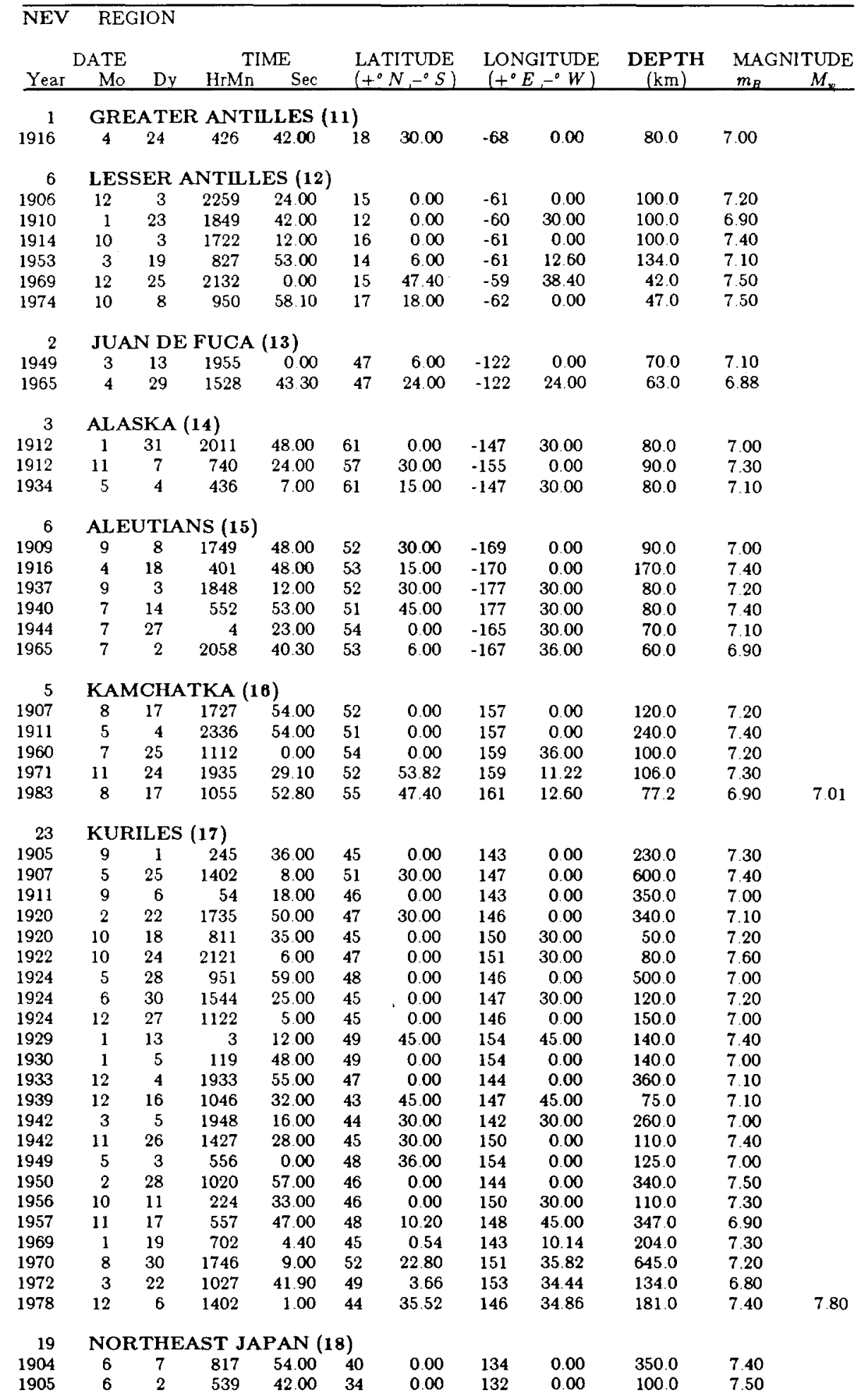




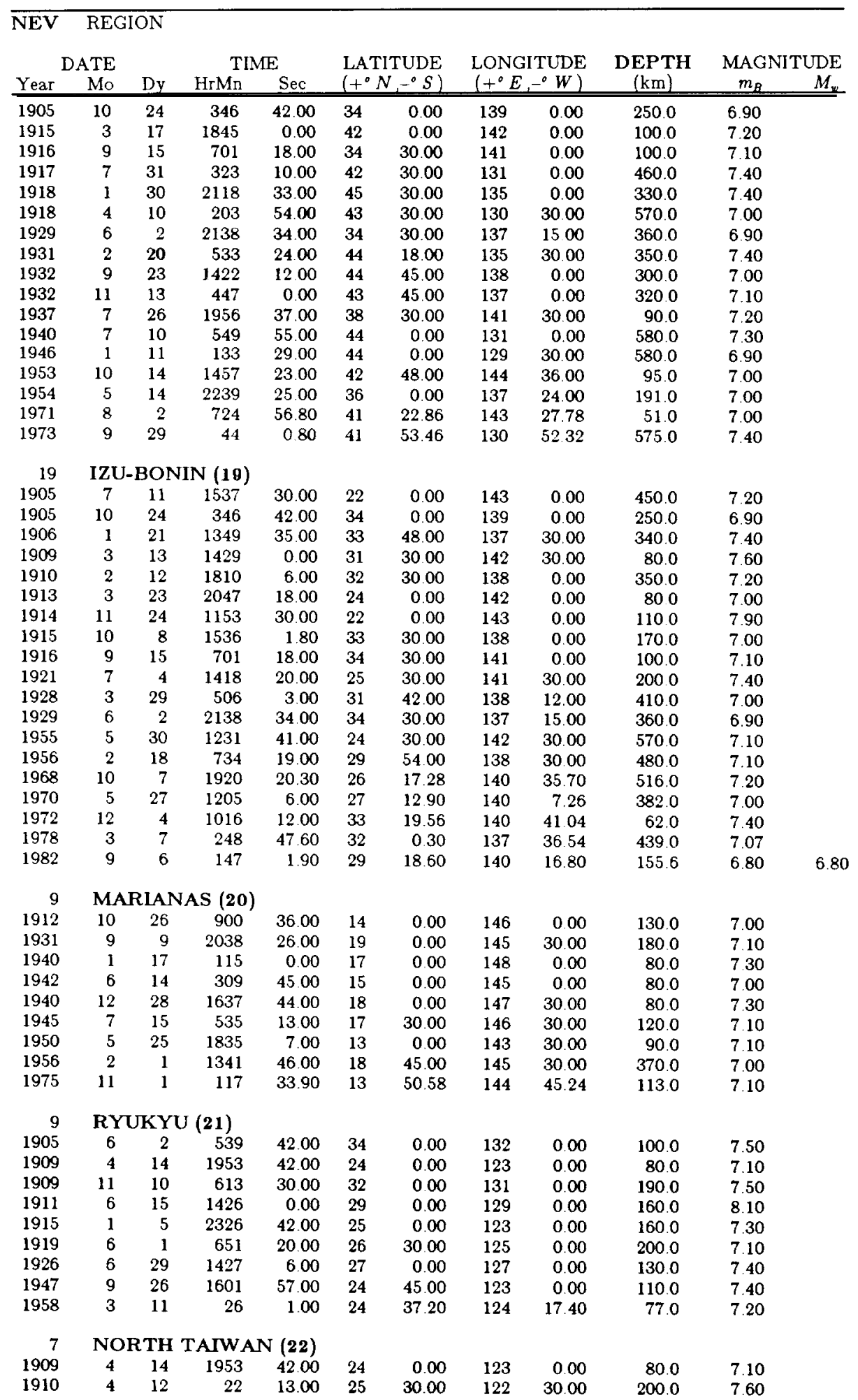


TABLE A (continued).

\begin{tabular}{|c|c|c|c|c|c|c|c|c|c|c|c|}
\hline \multicolumn{12}{|c|}{$\begin{array}{ll}\text { NEV } & \text { REGION }\end{array}$} \\
\hline \multicolumn{3}{|c|}{ DATE } & \multicolumn{2}{|c|}{ TIME } & \multirow{2}{*}{\multicolumn{2}{|c|}{$\begin{array}{l}\text { LATITUDE } \\
\left(+^{\circ} N-^{0} S\right)\end{array}$}} & \multirow{2}{*}{\multicolumn{2}{|c|}{$\begin{array}{l}\text { LONGITUDE } \\
\left(+^{\circ} E,-^{0} W\right)\end{array}$}} & \multirow{2}{*}{$\underset{(\mathrm{km})}{\text { DEPTH }}$} & \multicolumn{2}{|c|}{ MAGNITUDE } \\
\hline Year & Mo & Dy & $\mathrm{HrMn}$ & $\mathrm{Sec}$ & & & & & & & $M_{*}$ \\
\hline 1915 & 1 & 5 & 2326 & 42.00 & 25 & 0.00 & 123 & 0.00 & 160.0 & 7.30 & \\
\hline 1947 & 9 & 26 & 1601 & 57.00 & 24 & 45.00 & 123 & 0.00 & 110.0 & 7.40 & \\
\hline 1959 & 4 & 26 & 2040 & 37.00 & 24 & 51.00 & 122 & 4500 & 113.0 & 7.50 & \\
\hline 1963 & 2 & 13 & 850 & 4.50 & 24 & 30.00 & 122 & 6.00 & 67.0 & 7.25 & \\
\hline 1967 & 10 & 25 & 59 & 2260 & 24 & 30.00 & 122 & 12.00 & 65.0 & 7.00 & \\
\hline \multicolumn{12}{|c|}{ LUZON (23) } \\
\hline 1930 & 12 & 21 & 1451 & 24.00 & 20 & 0.00 & 122 & 15.00 & 170.0 & 7.00 & \\
\hline 1938 & 5 & 23 & 821 & 53.00 & 18 & 0.00 & 119 & 30.00 & 80.0 & 7.00 & \\
\hline \multicolumn{12}{|c|}{ PHILIPPINES (24) } \\
\hline 1907 & 3 & 29 & 2046 & 30.00 & 3 & 0.00 & 122 & 0.00 & 500.0 & 7.20 & \\
\hline 1907 & 6 & 25 & 1754 & 36.00 & 1 & 0.00 & 127 & 0.00 & 200.0 & 7.50 & \\
\hline 1918 & 2 & 7 & 520 & 30.00 & 6 & 30.00 & 126 & 30.00 & 120.0 & 7.20 & \\
\hline 1928 & 8 & 12 & 808 & 50.00 & 1 & 0.00 & 127 & 0.00 & 130.0 & 7.00 & \\
\hline 1936 & 1 & 20 & 1656 & 19.00 & 6 & 0.00 & 127 & 0.00 & 80.0 & 7.00 & \\
\hline 1941 & 2 & 4 & 1403 & 12.00 & 9 & 0.00 & 124 & 0.00 & 600.0 & 7.00 & \\
\hline 1948 & 1 & 28 & 347 & 21.00 & -1 & 30.00 & 126 & 30.00 & 80.0 & 7.10 & \\
\hline 1950 & 7 & 29 & 1646 & 2.00 & 2 & 30.00 & 127 & 0.00 & 80.0 & 7.00 & \\
\hline 1949 & 4 & 30 & 123 & 32.00 & 6 & 30.00 & 125 & 0.00 & 130.0 & 7.30 & \\
\hline 1955 & 3 & 31 & 1817 & 19.00 & 8 & 6.00 & 123 & 12.00 & 96.0 & 6.90 & \\
\hline 1972 & 6 & 11 & 1641 & 0.90 & 3 & 56.40 & 124 & 19.08 & 325.0 & 7.40 & \\
\hline 1966 & 9 & 8 & 2115 & 50.50 & 2 & 24.00 & 128 & 18.00 & 71.0 & 7.20 & \\
\hline 1969 & 1 & 30 & 1029 & 40.40 & 4 & 48.30 & 127 & 26.22 & 70.0 & 7.10 & \\
\hline 1970 & 1 & 10 & 1207 & 8.60 & 6 & 49.50 & 126 & 44.22 & 73.0 & 7.30 & \\
\hline 1970 & 3 & 30 & 1646 & 45.60 & 6 & 47.82 & 126 & 39.06 & 76.0 & 7.10 & \\
\hline 1975 & 7 & 10 & 1829 & 16.00 & 6 & 30.42 & 126 & 38.52 & 86.0 & 7.00 & \\
\hline 1976 & 11 & 7 & 1709 & 6.10 & 8 & 28.68 & 126 & 22.50 & 60.0 & 6.90 & \\
\hline 1984 & 11 & 20 & 815 & 16.23 & 5 & 10.02 & 125 & 7.44 & 180.7 & 7.20 & 7.48 \\
\hline \multicolumn{12}{|c|}{ SULAWESI (25) } \\
\hline 1905 & 1 & 22 & 243 & 54.00 & 1 & 0.00 & 123 & 0.00 & 90.0 & 7.80 & \\
\hline 1939 & 12 & 21 & 2100 & 40.00 & 0 & 0.00 & 123 & 0.00 & 150.0 & 7.80 & \\
\hline 1941 & 9 & 17 & 647 & 57.00 & 0 & -30.00 & 121 & 30.00 & 190.0 & 6.90 & \\
\hline 1942 & 5 & 28 & 101 & 48.00 & 0 & 0.00 & 124 & 0.00 & 1200 & 7.40 & \\
\hline 1948 & 2 & 9 & 1454 & 22.00 & 0 & 0.00 & 122 & 30.00 & 160.0 & 6.90 & \\
\hline 1961 & 3 & 28 & 935 & 55.00 & 0 & 12.00 & 123 & 36.00 & 83.0 & 7.30 & \\
\hline \multicolumn{12}{|c|}{ BURMA (28) } \\
\hline 1906 & 8 & 31 & 1457 & 30.00 & 27 & 0.00 & 97 & 0.00 & 100.0 & 6.70 & \\
\hline 1932 & 8 & 14 & 439 & 32.00 & 26 & 0.00 & 95 & 30.00 & 120.0 & 7.00 & \\
\hline 1954 & 3 & 21 & 2342 & 0.00 & 24 & 30.00 & 95 & 0.00 & 180.0 & 7.40 & \\
\hline \multicolumn{12}{|c|}{ ANDAMAN (27) } \\
\hline 1914 & 10 & 11 & 1617 & 6.00 & 12 & 0.00 & 94 & 0.00 & 80.0 & 7.20 & \\
\hline 1916 & 7 & 27 & 1152 & 42.00 & 4 & 0.00 & 96 & 30.00 & 100.0 & 7.00 & \\
\hline 1983 & 4 & 4 & 251 & 34.90 & 5 & 43.80 & 94 & 48.60 & 71.6 & 6.96 & \\
\hline \multicolumn{12}{|c|}{ SUNDA (28) } \\
\hline 1913 & 8 & 13 & 425 & 42.00 & -5 & 30.00 & 105 & 0.00 & 75.0 & 7.30 & \\
\hline 1943 & $\cdot 11$ & 26 & 2125 & 22.00 & -2 & 30.00 & 100 & 0.00 & 130.0 & 7.10 & \\
\hline 1957 & 4 & 16 & 404 & 3.00 & -4 & 40.80 & 107 & 9.60 & 546.0 & 7.20 & \\
\hline \multicolumn{12}{|c|}{ JAVA (29) } \\
\hline 1911 & 7 & 5 & 1840 & 6.00 & -7 & 30.00 & 117 & 30.00 & 370.0 & 7.00 & \\
\hline 1916 & 9 & 11 & 630 & 36.00 & -9 & 0.00 & 113 & 0.00 & 100.0 & 7. 10 & \\
\hline 1926 & 9 & 10 & 1034 & 29.00 & -9 & 0.00 & 111 & 0.00 & 80.0 & 7.00 & \\
\hline 1937 & 8 & 11 & 55 & 54.00 & -6 & 15.00 & 116 & 30.00 & 610.0 & 7.20 & \\
\hline 1943 & 7 & 23 & 1453 & 9.00 & -9 & 30.00 & 110 & 0.00 & 90.0 & 760 & \\
\hline 1949 & 4 & 23 & 1115 & 39.00 & -8 & 0.00 & 121 & 0.00 & 80.0 & 7.00 & \\
\hline 19 & TIM & $\mathbf{R}$ & & & & & & & & & \\
\hline 1909 & 5 & 30 & 2101 & 18.00 & -8 & 0.00 & 131 & 0.00 & 100.0 & 7.10 & \\
\hline 1914 & 7 & 4 & 2338 & 54.00 & -5 & 30.00 & 129 & 0.00 & 200.0 & 7.10 & \\
\hline
\end{tabular}




\begin{tabular}{|c|c|c|c|c|c|c|c|c|c|c|c|}
\hline \multirow{2}{*}{$\begin{array}{l}\text { NEV } \\
\text { Year }\end{array}$} & \multicolumn{11}{|l|}{ REGION } \\
\hline & $\begin{array}{r}\text { DATE } \\
\text { Mo }\end{array}$ & Dy & \multicolumn{2}{|c|}{ TIME } & \multicolumn{2}{|c|}{$\begin{array}{l}\text { LATITUDE } \\
\left(+^{\circ} N-^{0} S\right)\end{array}$} & \multicolumn{2}{|c|}{$\begin{array}{l}\text { LONGITUDE } \\
\left(+^{\circ} E-_{-}^{\circ} W\right)\end{array}$} & $\begin{array}{c}\text { DEPTH } \\
(\mathrm{km})\end{array}$ & \multicolumn{2}{|c|}{ MAGNITUDE } \\
\hline 1917 & 8 & 30 & 407 & 15.00 & -7 & 30.00 & 128 & 0.00 & 100.0 & 7.30 & \\
\hline 1918 & 11 & 18 & 1841 & 55.00 & -7 & 0.00 & 129 & 0.00 & 190.0 & 7.50 & \\
\hline 1918 & 11 & 23 & 2257 & 55.00 & -7 & 0.00 & 129 & 0.00 & 190.0 & 7.10 & \\
\hline 1927 & 6 & 3 & 712 & 1100 & -7 & 0.00 & 131 & 0.00 & 150.0 & 7.20 & \\
\hline 1931 & 3 & 28 & 1238 & 37.00 & -7 & 0.00 & 129 & 30.00 & 80.0 & 7.20 & \\
\hline 1934 & 6 & 29 & 825 & 17.00 & -6 & 45.00 & 123 & 45.00 & 720.0 & 7.00 & \\
\hline 1938 & 10 & 20 & 219 & 27.00 & -9 & 0.00 & 123 & 0.00 & 90.0 & 7.30 & \\
\hline 1944 & 3 & 22 & 43 & 18.00 & -8 & 30.00 & 123 & 30.00 & 220.0 & 7.20 & \\
\hline 1950 & 11 & 2 & 1527 & 56.00 & -6 & 30.00 & 129 & 30.00 & 50.0 & 740 & \\
\hline 1954 & 2 & 20 & 1835 & 500 & -6 & 45.00 & 124 & 30.00 & 580.0 & 7.00 & \\
\hline 1956 & 7 & 18 & 619 & 34.00 & -5 & 4.20 & 130 & 15.60 & 128.0 & 7.20 & \\
\hline 1957 & 3 & 23 & 512 & 31.00 & -5 & 30.00 & 131 & 0.00 & 100.0 & 7.10 & \\
\hline 1963 & 11 & 4 & 117 & 11.40 & -6 & 51.60 & 129 & 3480 & 100.0 & 780 & \\
\hline 1964 & 7 & 8 & 1155 & 39.10 & -5 & 30.00 & 129 & 48.00 & 165.0 & 7.00 & \\
\hline 1971 & 7 & 8 & 1907 & 7.30 & -7 & 1.86 & 129 & 41.76 & 92.0 & 7.30 & \\
\hline 1982 & 6 & 22 & 418 & 40.50 & -7 & 20.34 & 126 & 2.58 & 450.0 & 7.20 & 7.44 \\
\hline 1983 & 11 & 24 & 530 & 34.90 & -7 & 33.00 & 128 & 15.00 & 157.1 & 7.20 & 7.40 \\
\hline 14 & NEW GUINEA (31) & & & & & & & & & & \\
\hline 1936 & 6 & 10 & 823 & 21.00 & -5 & 30.00 & 147 & 0.00 & 190.0 & 7.00 & \\
\hline 1939 & 3 & 2 & 700 & 27.00 & -4 & 0.00 & 143 & 0.00 & 130.0 & 7.00 & \\
\hline 1943 & 12 & 1 & 604 & 55.00 & -4 & 45.00 & 144 & 0.00 & 120.0 & 7.30 & \\
\hline 1944 & 1 & 7 & 249 & 20.00 & -4 & 30.00 & 143 & 30.00 & 120.0 & 7.00 & \\
\hline 1946 & 1 & 17 & 939 & 35.00 & -7 & 30.00 & 147 & 30.00 & 100.0 & 7.20 & \\
\hline 1946 & 9 & 23 & 2330 & 0.00 & -6 & 0.00 & 145 & 0.00 & 100.0 & 7.10 & \\
\hline 1950 & 2 & 17 & 2107 & 700 & -7 & 0.00 & 146 & 0.00 & 180.0 & 7.20 & \\
\hline 1951 & 2 & 17 & 2107 & 7.00 & .7 & 0.00 & 146 & 0.00 & 1800 & 6.90 & \\
\hline 1959 & 11 & 19 & 1108 & 41.00 & -5 & 30.00 & 146 & 0.00 & 100.0 & 7.00 & \\
\hline 1961 & 1 & 5 & 1553 & 56.00 & -4 & 6.00 & 143 & 0.00 & 108.0 & 7.10 & \\
\hline 1963 & 2 & 26 & 2014 & 1000 & -7 & 36.00 & 146 & 12.00 & 182.0 & 7.30 & \\
\hline 1968 & 5 & 28 & 1327 & 1870 & -2 & 54.84 & 139 & 19.14 & 65.0 & 7.20 & \\
\hline 1975 & 12 & 25 & 2322 & 21.70 & -4 & 5.04 & 142 & 2.40 & 115.0 & 6.80 & \\
\hline 1980 & 7 & 16 & 1956 & 46.70 & -4 & 27.36 & 143 & 31.26 & 81.9 & 720 & 7.26 \\
\hline 4 & NEW BRITAIN (32) & & & & & & & & & & \\
\hline 1910 & 9 & 7 & 711 & 18.00 & -6 & 0.00 & 151 & 0.00 & 80.0 & 7.20 & \\
\hline 1923 & 11 & 2 & 2108 & 6.00 & -4 & 30.00 & 151 & 30.00 & 50.0 & 7.20 & \\
\hline 1946 & 1 & 17 & 939 & 35.00 & -7 & 30.00 & 147 & 30.00 & 100.0 & 7.20 & \\
\hline 1973 & 1 & 18 & 928 & 14.10 & -6 & 52.08 & 149 & 59.58 & 43.0 & 6.80 & \\
\hline 10 & NEW IRELAND (33) & & & & & & & & & & \\
\hline 1912 & 9 & 1 & 410 & 0.00 & -4 & 30.00 & 155 & 0.00 & 430.0 & 7.00 & \\
\hline 1928 & 3 & 13 & 1831 & 52.00 & -5 & 30.00 & 153 & 0.00 & 100.0 & 7.00 & \\
\hline 1932 & 1 & 9 & 1021 & 42.00 & -6 & 12.00 & 154 & 30.00 & 380.0 & 7.20 & \\
\hline 1941 & 9 & 4 & 1021 & 44.00 & -4 & 45.00 & 154 & 0.00 & 90.0 & 7.10 & \\
\hline 1943 & 12 & 23 & 1900 & 10.00 & -5 & 30.00 & 153 & 30.00 & 50.0 & 7.20 & \\
\hline 1951 & 5 & 21 & 827 & 20.00 & -6 & 0.00 & 154 & 30.00 & 150.0 & 7.00 & \\
\hline 1950 & 7 & 29 & 2349 & 2.00 & -6 & 30.00 & 155 & 0.00 & 70.0 & 6.90 & \\
\hline 1950 & 12 & 4 & 1628 & 3.00 & -5 & 0.00 & 153 & 30.00 & 110.0 & 7.30 & \\
\hline 1971 & 7 & 19 & 14 & 45.30 & -5 & 41.28 & 153 & 48.00 & 42.0 & 7.10 & \\
\hline 1983 & 3 & 18 & 905 & 50.10 & -4 & 51.60 & 153 & 30.60 & 69.9 & 7.30 & 7.71 \\
\hline 5 & SOLOMONS (34) & & & & & & & & & & \\
\hline 1937 & 9 & 15 & 1227 & 32.00 & -10 & 30.00 & 161 & 30.00 & 80.0 & 7.20 & \\
\hline 1950 & 7 & 29 & 2349 & 2.00 & -6 & 30.00 & 155 & 0.00 & 70.0 & 6.90 & \\
\hline 1955 & 10 & 13 & 926 & 49.00 & -10 & 0.00 & 160 & 45.00 & 70.0 & 7.10 & \\
\hline 1969 & 1 & 5 & 1326 & 39.90 & -7 & 58.50 & 158 & 54.54 & 71.0 & 7.30 & \\
\hline 1970 & 12 & 29 & 226 & 12.20 & -10 & 32.82 & 161 & 24.18 & 72.0 & 6.80 & \\
\hline 48 & NEW HEBRIDES (35) & & & & & & & & & & \\
\hline 1909 & 8 & 18 & 39 & 30.00 & -22 & 0.00 & 172 & 0.00 & 1000 & 7.20 & \\
\hline 1910 & 3 & 30 & 1655 & 48.00 & -21 & 0.00 & 170 & 0.00 & 80.0 & 7.10 & \\
\hline
\end{tabular}


TABLE A (continued).

\begin{tabular}{|c|c|c|c|c|c|c|c|c|c|c|c|}
\hline \multicolumn{12}{|c|}{$\begin{array}{ll}\text { NEV } & \text { REGION }\end{array}$} \\
\hline \multicolumn{3}{|c|}{ DATE } & \multicolumn{2}{|c|}{ TIME } & \multirow{2}{*}{\multicolumn{2}{|c|}{$\begin{array}{l}\text { LATITUDE } \\
\left(++^{0} N-{ }^{\circ} S\right)\end{array}$}} & \multirow{2}{*}{\multicolumn{2}{|c|}{$\begin{array}{l}\text { LONGITUDE } \\
\left(+{ }^{\circ} E-{ }^{\circ} W\right)\end{array}$}} & \multirow{2}{*}{$\begin{array}{c}\text { DEPTH } \\
(\mathrm{km})\end{array}$} & \multicolumn{2}{|c|}{ MAGNITUDE } \\
\hline Year & Mo & Dy & $\mathrm{HrMn}$ & $\mathrm{Sec}$ & & & & & & & \\
\hline $1910^{\circ}$ & 5 & 1 & 1830 & 36.00 & -20 & 0.00 & 169 & 0.00 & 800 & 7.10 & \\
\hline 1910 & 6 & 1 & 555 & 30.00 & -20 & 0.00 & 169 & 0.00 & 800 & 7.30 & \\
\hline 1910 & 6 & 1 & 648 & 18.00 & -20 & 0.00 & 169 & 0.00 & 80.0 & 7.10 & \\
\hline 1910 & 6 & 16 & 630 & 42.00 & -19 & 0.00 & 169 & 30.00 & 100.0 & 7.90 & \\
\hline 1910 & 11 & 9 & 602 & 0.00 & -15 & 000 & 166 & 0.00 & 80.0 & 7.50 & \\
\hline 1910 & 11 & 10 & 1219 & 54.00 & -14 & 0.00 & 166 & 30.00 & 90.0 & 7.10 & \\
\hline 1911 & 10 & 20 & 1744 & 0.00 & -12 & 30.00 & 166 & 0.00 & 160.0 & 7.00 & \\
\hline 1911 & 11 & 22 & 2305 & 24.00 & -15 & 0.00 & 169 & 0.00 & 200.0 & 7.30 & \\
\hline 1912 & 8 & 6 & 2111 & 18.00 & -14 & 0.00 & 167 & 0.00 & 260.0 & 7.30 & \\
\hline 1913 & 10 & 14 & 808 & 48.00 & -19 & 30.00 & 169 & 0.00 & 230.0 & 760 & \\
\hline 1913 & 11 & 10 & 2112 & 30.00 & -18 & 0.00 & 169 & 0.00 & 80.0 & 7.20 & \\
\hline 1913 & 11 & 15 & 527 & 6.00 & -23 & 0.00 & 171 & 0.00 & 150.0 & 7.10 & \\
\hline 1915 & 1 & 5 & 1433 & 15.00 & -15 & 0.00 & 168 & 0.00 & 200.0 & 7.30 & \\
\hline 1919 & 8 & 31 & 1720 & 46.00 & -16 & 0.00 & 169 & 0.00 & 180.0 & 7.30 & \\
\hline 1925 & $\mathbf{3}$ & & & & & & & & & & \\
\hline 1926 & 6 & 3 & 446 & 56.00 & -15 & 0.00 & 168 & 30.00 & 60.0 & 7.10 & \\
\hline 1928 & 8 & 24 & 2143 & 30.00 & -15 & 0.00 & 168 & 0.00 & 220.0 & 7.00 & \\
\hline 1933 & 1 & 1 & 848 & 39.00 & -14 & 45.00 & 168 & 0.00 & 140.0 & 7.00 & \\
\hline 1935 & 6 & 24 & 2323 & 14.00 & -15 & 45.00 & 167 & 45.00 & 140.0 & 7.10 & \\
\hline 1935 & 8 & 17 & 144 & 42.00 & -22 & 30.00 & 171 & 0.00 & 120.0 & 7.20 & \\
\hline 1937 & 7 & 2 & 237 & 15.00 & -14 & 15.00 & 167 & 0.00 & 80.0 & 7.00 & \\
\hline 1939 & 4 & 5 & 1642 & 40.00 & -19 & 30.00 & 168 & 0.00 & 70.0 & 7.00 & \\
\hline 1939 & 8 & 12 & 207 & 27.00 & -16 & 15.00 & 168 & 30.00 & 180.0 & 7.00 & \\
\hline 1940 & 1 & 6 & 1403 & 24.00 & -22 & 0.00 & 171 & 0.00 & 90.0 & 7.20 & \\
\hline 1940 & 2 & 20 & 218 & 20.00 & -13 & 30.00 & 167 & 0.00 & 200.0 & 7.00 & \\
\hline 1940 & 9 & 19 & 1819 & 48.00 & -24 & 0.00 & 171 & 0.00 & 80.0 & 7.00 & \\
\hline 1942 & 1 & 29 & 923 & 44.00 & -19 & 0.00 & 169 & 0.00 & 130.0 & 7.00 & \\
\hline 1944 & 10 & 5 & 1728 & 27.00 & -22 & 30.00 & 172 & 0.00 & 120.0 & 7.30 & \\
\hline 1944 & 11 & 24 & 449 & 3.00 & -19 & 0.00 & 169 & 0.00 & 170.0 & 7.40 & \\
\hline 1947 & 11 & 9 & 457 & 50.00 & -22 & 30.00 & 170 & 0.00 & 50.0 & 7.00 & \\
\hline 1949 & 7 & 23 & 1026 & 45.00 & -18 & 30.00 & 170 & 0.00 & 150.0 & 7.20 & \\
\hline 1950 & 9 & 10 & 1516 & 8.00 & -15 & 30.00 & 167 & 0.00 & 100.0 & 7.10 & \\
\hline 1951 & 3 & 10 & 2157 & 29.00 & -15 & 0.00 & 167 & 30.00 & 130.0 & 7.20 & \\
\hline 1951 & 3 & 24 & 17 & 36.00 & -10 & 30.00 & 166 & 0.00 & 150.0 & 7.10 & \\
\hline 1953 & 7 & 2 & 656 & 59.00 & -19 & 0.00 & 169 & 0.00 & 223.0 & 7.40 & \\
\hline 1957 & 12 & 17 & 1350 & 20.00 & -12 & 22.20 & 166 & 43.80 & 120.0 & 7.20 & \\
\hline 1960 & 3 & 8 & 1633 & 38.00 & -16 & 30.00 & 168 & 3000 & 250.0 & 7.20 & \\
\hline 1963 & 5 & 1 & 1003 & 20.20 & -19 & 0.00 & 168 & 54.00 & 142.0 & 7.10 & \\
\hline 1964 & 7 & 9 & 1639 & 49.30 & -15 & 30.00 & 167 & 36.00 & 127.0 & 7.40 & \\
\hline 1971 & 11 & 21 & 557 & 11.90 & -11 & 49.02 & 166 & 31.92 & 115.0 & 7.40 & \\
\hline 1972 & 2 & 14 & 2329 & 51.70 & -11 & 21.54 & 166 & 20.10 & 102.0 & 7.00 & \\
\hline 1973 & 12 & 29 & 19 & 31.10 & -15 & 7.02 & 166 & 53.76 & 47.0 & 7.20 & \\
\hline 1978 & 5 & 1 & 1303 & 37.10 & -21 & 14.40 & 169 & 48.00 & 73.4 & 7.10 & 7.37 \\
\hline 1978 & 5 & 13 & 708 & 46.20 & -14 & 31.26 & 167 & 19.14 & 180.4 & 6.80 & 680 \\
\hline 1981 & 7 & 6 & 308 & 24.70 & -22 & 15.60 & 171 & 43.80 & 58.3 & 7.30 & 7.54 \\
\hline 1984 & 4 & 6 & 2308 & 22.30 & -18 & 54.18 & 168 & 51.00 & 175.0 & 6.80 & 6.80 \\
\hline 45 & TOI & GA & & & & & & & & & \\
\hline 1907 & 3 & 31 & 2200 & 36.00 & -18 & 0.00 & -177 & 0.00 & 400.0 & 7.20 & \\
\hline 1909 & 2 & 22 & 921 & 42.00 & -18 & 0.00 & -179 & 0.00 & 550.0 & 7.60 & \\
\hline 1909 & 8 & 18 & 39 & 30.00 & -22 & 0.00 & 172 & 0.00 & 100.0 & 7.20 & \\
\hline 1910 & 3 & 30 & 1655 & 48.00 & -21 & 0.00 & 170 & 0.00 & 80.0 & 7.10 & \\
\hline 1910 & 8 & 21 & 538 & 36.00 & -17 & 0.00 & -179 & 0.00 & 600.0 & 7.40 & \\
\hline 1911 & 8 & 21 & 1628 & 55.00 & -21 & 0.00 & -176 & 0.00 & 300.0 & 7.20 & \\
\hline 1913 & 11 & 15 & 527 & 6.00 & -23 & 0.00 & 171 & 0.00 & 150.0 & 7.10 & \\
\hline 1915 & 2 & 25 & 2036 & 12.00 & -20 & 0.00 & 180 & 0.00 & 6000 & 7.10 & \\
\hline 1916 & 7 & 8 & 934 & 30.00 & .18 & 0.00 & 180 & 0.00 & 600.0 & 7.10 & \\
\hline 1919 & 1 & 1 & 259 & 57.00 & -19 & 30.00 & -176 & 30.00 & 180.0 & 7.70 & \\
\hline 1919 & 8 & 18 & 1655 & 25.00 & -20 & 30.00 & -178 & 30.00 & 3000 & 7.00 & \\
\hline 1924 & 1 & 16 & 2138 & 0.00 & -21 & 0.00 & -176 & 0.00 & 350.0 & 7.00 & \\
\hline 1924 & 5 & 4 & 1651 & 43.00 & -21 & 0.00 & -178 & 0.00 & 560.0 & 7.20 & \\
\hline 1927 & 4 & 1 & 1906 & 9.00 & -20 & 0.00 & -177 & 30.00 & 400.0 & 7.00 & \\
\hline 1932 & 5 & 26 & 1609 & 40.00 & -25 & 30.00 & 179 & 15.00 & 600.0 & 7.50 & \\
\hline 1933 & 9 & 6 & 2208 & 29.00 & -21 & 30.00 & -179 & 45.00 & 600.0 & 7.00 & \\
\hline 1934 & 10 & 10 & 1542 & 6.00 & -23 & 30.00 & 180 & 0.00 & 540.0 & 7.20 & \\
\hline 1935 & 7 & 29 & 738 & 53.00 & -20 & 45.00 & -178 & 0.00 & 510.0 & 7.10 & \\
\hline
\end{tabular}




\begin{tabular}{|c|c|c|c|c|c|c|c|c|c|c|c|}
\hline \multicolumn{3}{|c|}{ DATE } & \multicolumn{2}{|c|}{ TIME } & \multicolumn{2}{|c|}{$\begin{array}{l}\text { LATITUDE } \\
\left(+^{\circ} N-^{\circ} S\right)\end{array}$} & \multicolumn{2}{|c|}{$\begin{array}{l}\text { LONGITUDE } \\
\left(+^{\circ} E,-^{\circ} W\right)\end{array}$} & $\begin{array}{c}\text { DEPTH } \\
(\mathrm{km})\end{array}$ & \multicolumn{2}{|c|}{ MAGNITUDE } \\
\hline 1935 & 8 & 17 & 144 & 42.00 & -22 & 30.00 & 171 & 0.00 & 120.0 & 7.20 & \\
\hline 1937 & 4 & 16 & 301 & 37.00 & -21 & 30.00 & -177 & 0.00 & 400.0 & 7.50 & \\
\hline 1940 & $i$ & 6 & 1403 & 2400 & -22 & 0.00 & 171 & 0.00 & 90.0 & 7.20 & \\
\hline 1940 & 9 & 19 & 1819 & 4800 & -24 & 0.00 & 171 & 0.00 & 80.0 & 7.00 & \\
\hline 1941 & 11 & 24 & 2146 & 23.00 & -28 & 0.00 & -177 & 30.00 & 80.0 & 7.00 & \\
\hline 1943 & 9 & 27 & 2203 & 4400 & -30 & 0.00 & -178 & 0.00 & 90.0 & 7.00 & \\
\hline 1944 & 5 & 25 & 106 & 3700 & -21 & 30.00 & -179 & 30.00 & 640.0 & 7.00 & \\
\hline 1944 & 10 & 5 & 1728 & 27.00 & -22 & 30.00 & 172 & 0.00 & 1200 & 7.30 & \\
\hline 1947 & 11 & 9 & 457 & 50.00 & -22 & 30.00 & 170 & 0.00 & 50.0 & 7.00 & \\
\hline 1948 & 1 & 27 & 1158 & 28.00 & -20 & 30.00 & -178 & 0.00 & 6300 & 7.00 & \\
\hline 1949 & 7 & 23 & 1026 & 45.00 & -18 & 30.00 & 170 & 0.00 & 150.0 & 7.20 & \\
\hline 1949 & 11 & 22 & 51 & 49.00 & -28 & 30.00 & -178 & 30.00 & 180.0 & 7.10 & \\
\hline 1950 & 12 & 10 & 1323 & 4.00 & -28 & 0.00 & -178 & 30.00 & 250.0 & 7.20 & \\
\hline 1950 & 12 & 14 & 152 & 49.00 & -19 & 15.00 & -175 & 45.00 & 200.0 & 750 & \\
\hline 1955 & 8 & 6 & 831 & 28.00 & -21 & 0.00 & -178 & 0.00 & 360.0 & 7.00 & \\
\hline 1956 & 5 & 23 & 2048 & 28.20 & -15 & 24.60 & -178 & 43.80 & 396.0 & 7.20 & \\
\hline 1956 & 12 & 27 & 14 & 11.00 & -23 & 30.00 & -177 & 000 & 240.0 & 7.00 & \\
\hline 1957 & 7 & 14 & 623 & 52.00 & -27 & 0.00 & -178 & 0.00 & 150.0 & 7.00 & \\
\hline 1957 & 9 & 28 & 1420 & 0.00 & -20 & 28.80 & -178 & 30.60 & 549.0 & 7.20 & \\
\hline 1959 & 9 & 14 & 1409 & 50.00 & -28 & 40.20 & -177 & 42.60 & 73.0 & 7.30 & \\
\hline 1967 & 10 & 9 & 1721 & 46.00 & -21 & 600 & -179 & 12.00 & 605.0 & 7.10 & \\
\hline 1970 & 1 & 20 & 719 & 51.20 & -25 & 48.00 & -177 & 20.94 & 82.0 & 7.20 & \\
\hline 1972 & 3 & 30 & 534 & 50.00 & -25 & 42.00 & 179 & 36.00 & 479.0 & 7.00 & \\
\hline 1972 & 5 & 22 & 2045 & 57.30 & -17 & 41.34 & -175 & 11.34 & 227.0 & 7.20 & \\
\hline 1977 & 6 & 22 & 1208 & 33.40 & -22 & 52.68 & -175 & 54.00 & 59.1 & 7.60 & 8.04 \\
\hline 1980 & 4 & 13 & 1804 & 31.90 & -23 & 27.96 & -177 & 17.82 & 166.2 & 7.30 & 7.57 \\
\hline 1981 & 7 & 6 & 0308 & 24.70 & -4 & 27.36 & 143 & 31.26 & 81.0 & 7.10 & 7.26 \\
\hline & & $\mathrm{RMA}$ & DEC (3 & & & & & & & & \\
\hline 1953 & 9 & 29 & 136 & 46.00 & -36 & 54.00 & 177 & 6.00 & 287.0 & 7.00 & \\
\hline 1970 & 1 & 8 & 1712 & 39.10 & -34 & 44.46 & 178 & 34.08 & 179.0 & 7.00 & \\
\hline 1 & & $\mathrm{~W} \mathrm{ZE}$ & ALAND & (38) & & & & & & & \\
\hline 1942 & 8 & 1 & 1234 & 300 & -41 & 0.00 & 175 & 45.00 & 50.0 & 7.10 & \\
\hline 12 & & JDU-1 & KUSH (3 & & & & & & & & \\
\hline 1909 & 7 & 7 & 2137 & 50.00 & 36 & 30.00 & 70 & 30.00 & 230.0 & 7.60 & \\
\hline 1911 & 7 & 4 & 1333 & 26.00 & 36 & 0.00 & 70 & 30.00 & 190.0 & 7.40 & \\
\hline 1921 & 11 & 15 & 2036 & 38.00 & 36 & 30.00 & 70 & 30.00 & 215.0 & 7.60 & \\
\hline 1922 & 12 & 6 & 1355 & 36.00 & 36 & 30.00 & 70 & 30.00 & 230.0 & 7.30 & \\
\hline 1924 & 10 & 13 & 1617 & 45.00 & 36 & 0.00 & 70 & 30.00 & 220.0 & 7.20 & \\
\hline 1929 & 2 & 1 & 1714 & 26.00 & 36 & 30.00 & 70 & 30.00 & 220.0 & 7.00 & \\
\hline 1937 & 11 & 14 & 1058 & 12.00 & 36 & 30.00 & 70 & 30.00 & 240.0 & 7.20 & \\
\hline 1943 & 2 & 28 & 1254 & 33.00 & 36 & 30.00 & 70 & 30.00 & 210.0 & 7.10 & \\
\hline 1949 & 3 & 4 & 1019 & 25.00 & 36 & 0.00 & 70 & 30.00 & 2300 & 7.40 & \\
\hline 1965 & 3 & 14 & 1553 & 6.60 & 36 & 25.20 & 70 & 43.80 & 205.0 & 7.50 & \\
\hline 1974 & 7 & 30 & 512 & 40.60 & 36 & 21.18 & 70 & 45.78 & 2110 & 7.10 & \\
\hline 1983 & 12 & 30 & 2352 & 39.90 & 36 & 22.32 & 70 & 44.48 & 215.0 & 7.40 & \\
\hline 1 & & $N(4)$ & & & & & & & & & \\
\hline 1934 & 6 & 13 & 2210 & 28.00 & 27 & 30.00 & 62 & 30.00 & 80.0 & 7.00 & \\
\hline 4 & & $\mathrm{EECI}$ & (41) & & & & & & & & \\
\hline 1911 & 4 & 4 & 1543 & 54.00 & 36 & 30.00 & 25 & 30.00 & 140.0 & 7.00 & \\
\hline 1926 & 6 & 26 & 1946 & 34.00 & 36 & 30.00 & 27 & 30.00 & 100.0 & 7.70 & \\
\hline 1926 & 8 & 30 & 1138 & 12.00 & 36 & 45.00 & 23 & 15.00 & 100.0 & 7.10 & \\
\hline 1957 & 4 & 25 & 225 & 42.00 & 36 & 28.20 & 28 & 33.60 & 53.0 & 7.10 & \\
\hline 2 & & MAN & $A(42)$ & & & & & & & & \\
\hline 1940 & 11 & 10 & 139 & 900 & 45 & 45.00 & 26 & 30.00 & 150.0 & 7.30 & \\
\hline 1977 & 3 & 4 & 1921 & 54.10 & 45 & 46.32 & 26 & 45.66 & 94.0 & 7.20 & 7.40 \\
\hline 1 & & AIN ( & & & & & & & & & \\
\hline 1954 & 3 & $29^{\circ}$ & 617 & 500 & 37 & 0.00 & -3 & 36.00 & 640.0 & 7.00 & \\
\hline
\end{tabular}

Magnitudes are $m_{\mathrm{B}}$ for events that occurred prior to 1975 and $M_{\mathrm{W}}$ thereafter, where $m_{\mathrm{B}}=0.63 M_{\mathrm{W}}+2.5$ (Kanamori, 1983) 
138

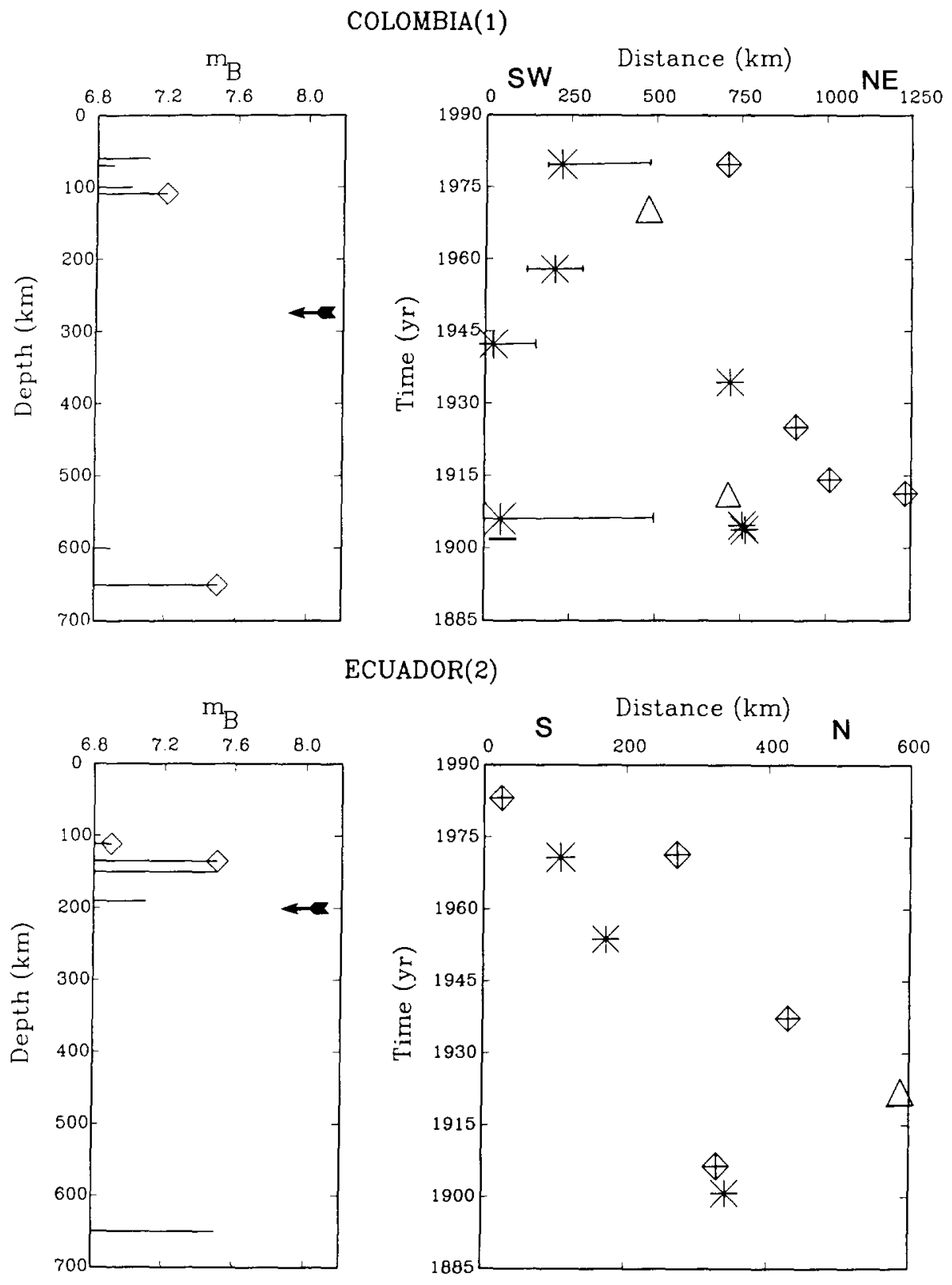

Fig. Al-1. 
PERU(3)
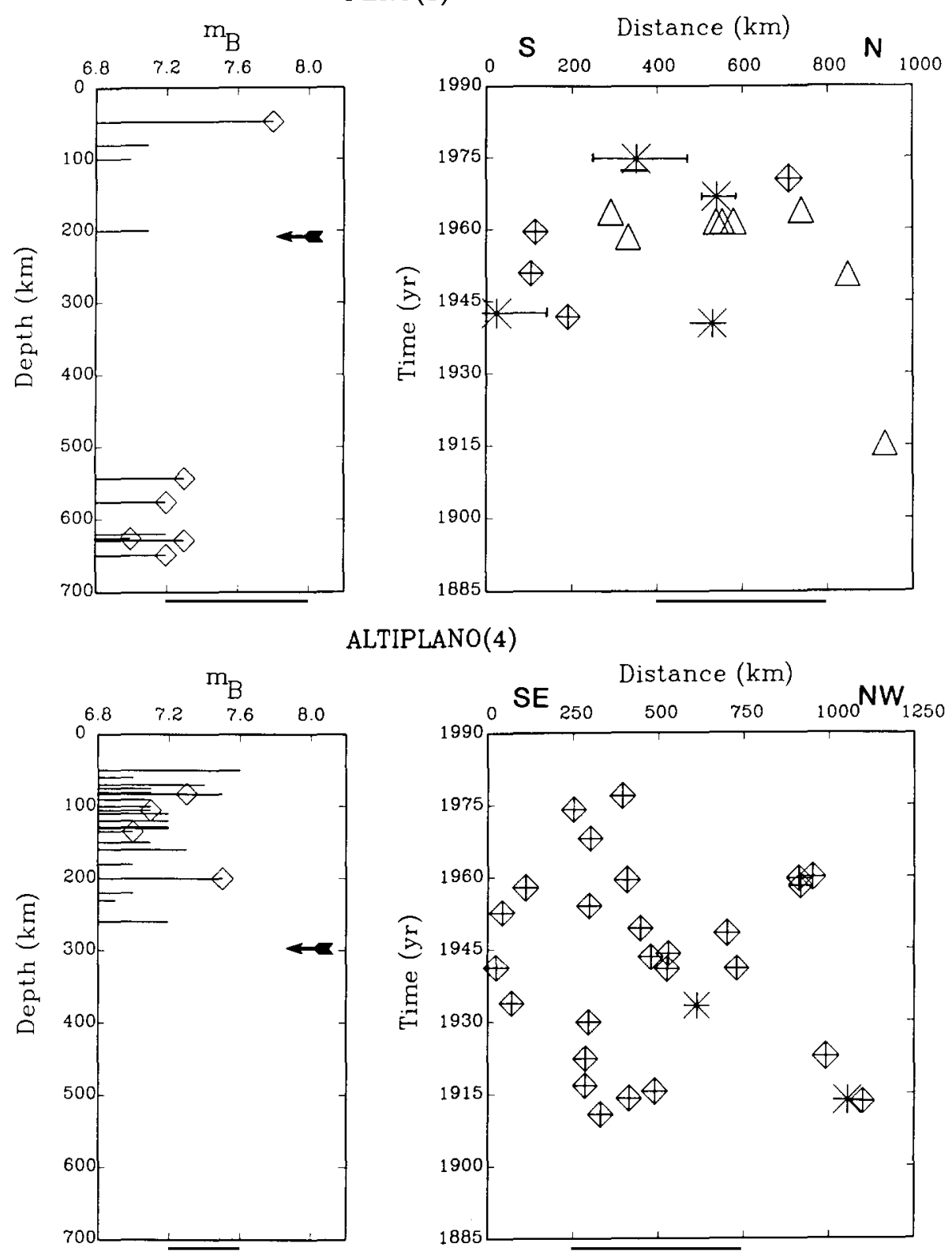

ALTIPLANO(4)

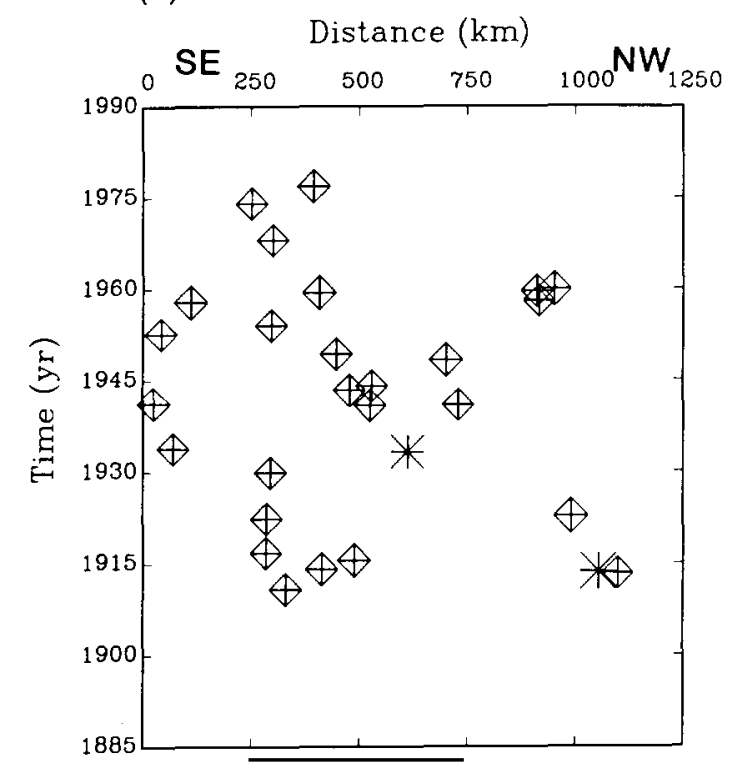

Fig. A1-2.

events shown in the left-hand side figure with $d \leq 300 \mathrm{~km}$ and triangles are deep events $(d>300$ $\mathrm{km}$ ). Symbol sizes are proportional to the magnitude of the events. For regions 39-42, the righthand figure displays the time distribution for each region of large (a) intermediate and deep focus earthquakes, and (b) shallow events with $M_{\mathrm{S}} \geq 7.5$. Deep focus earthquakes are indicated by a closed diamond in these figures. Note that the occurrence of intraplate large events in some regions such as the Altiplano (4), North Chile (5), Kuriles (17), Northeast Japan (18), Timor (30), New Hebrides 


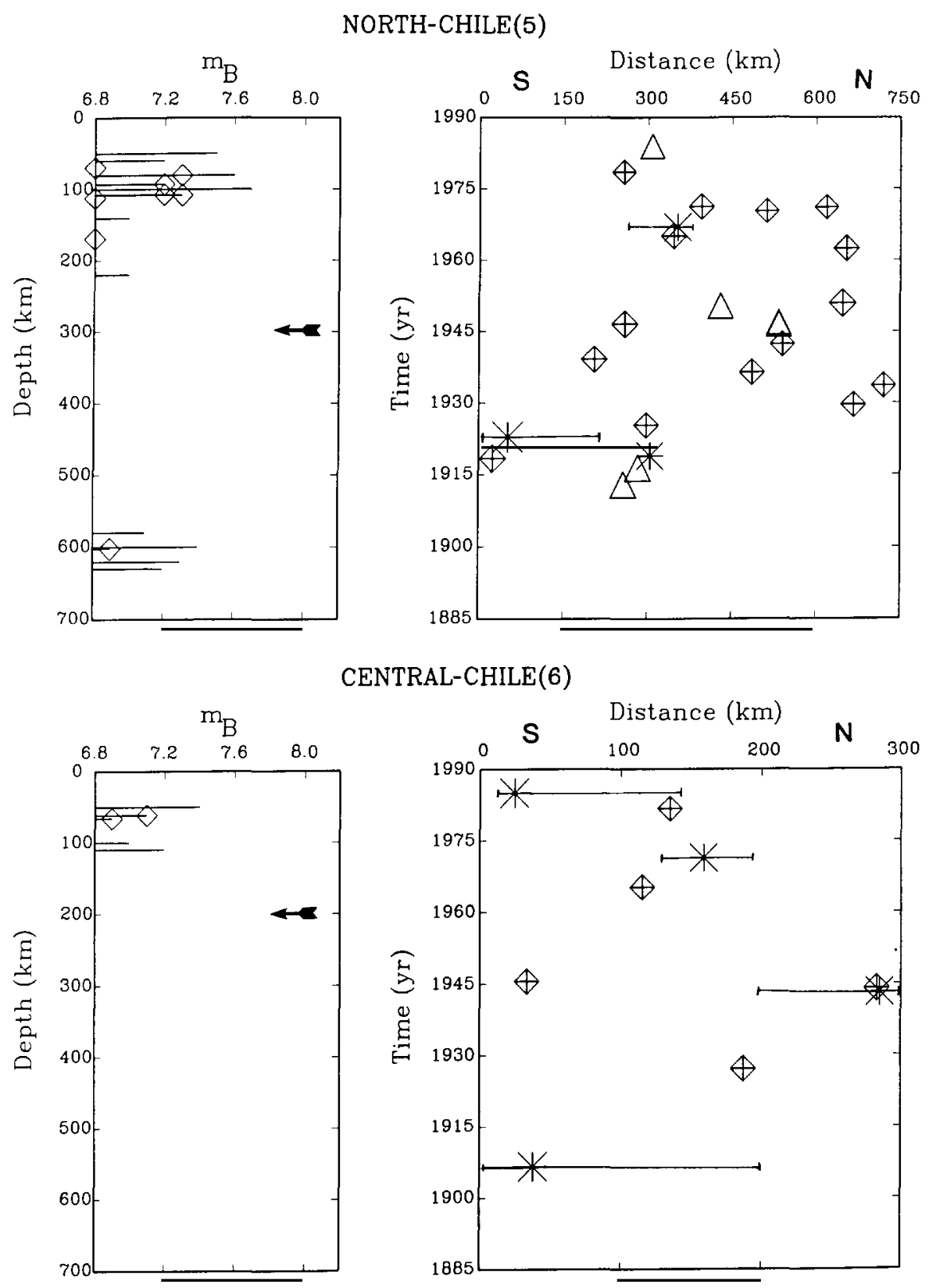

Fig. A1-3.

(35), Tonga (36), and the Hindu-Kush (39) regions have been relatively constant in the last 80 years. However, some other regions such as the Ryukyu (21) and Sulawesi (23) were especially active in large events in the early part of the century, whereas other regions such as Peru (3) and New Guinea (31) appear more active in recent decades. This text replaces figure captions. 
141

SOUTH-CHILE(7)

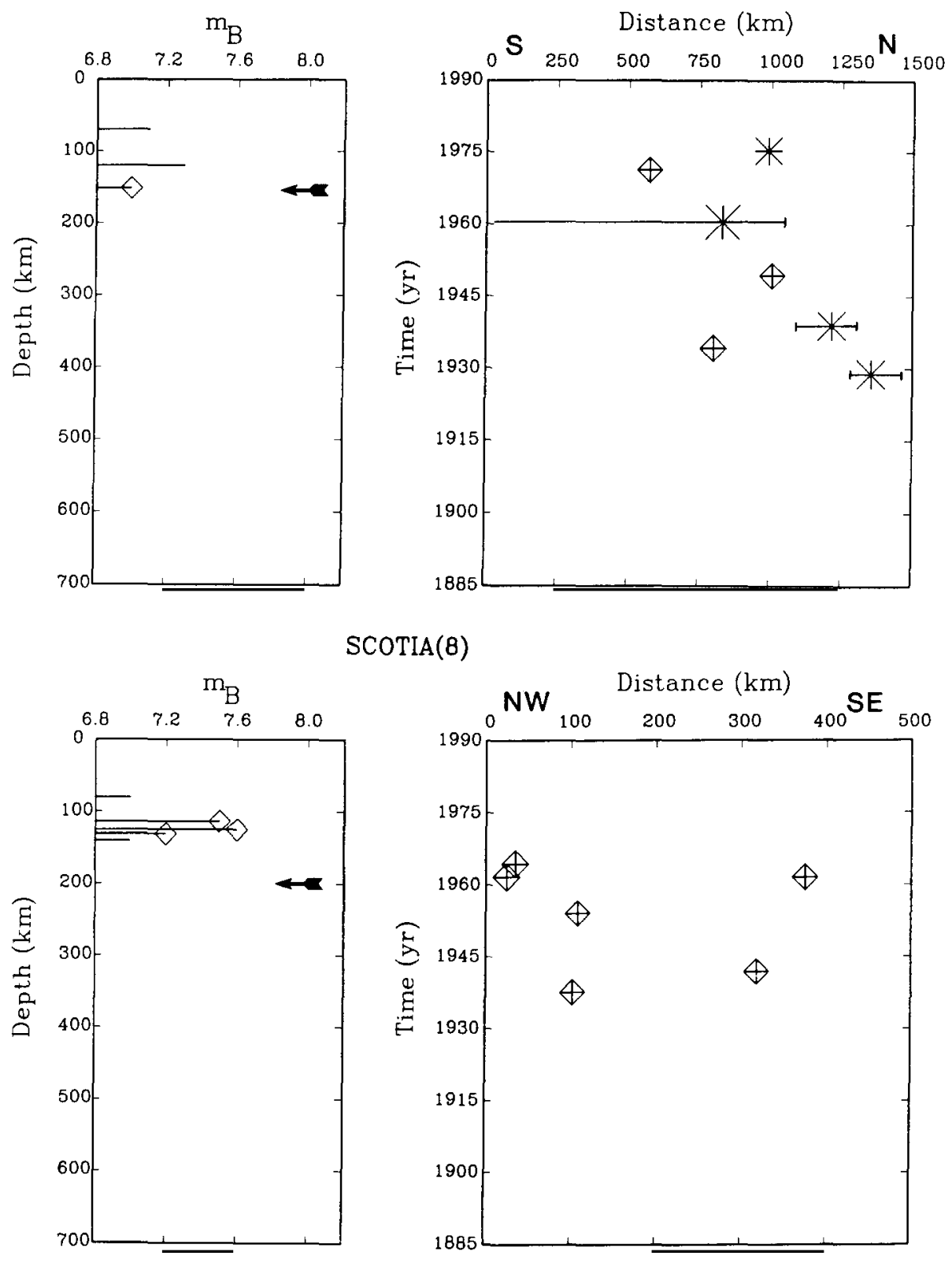

Fig. A1-4. 

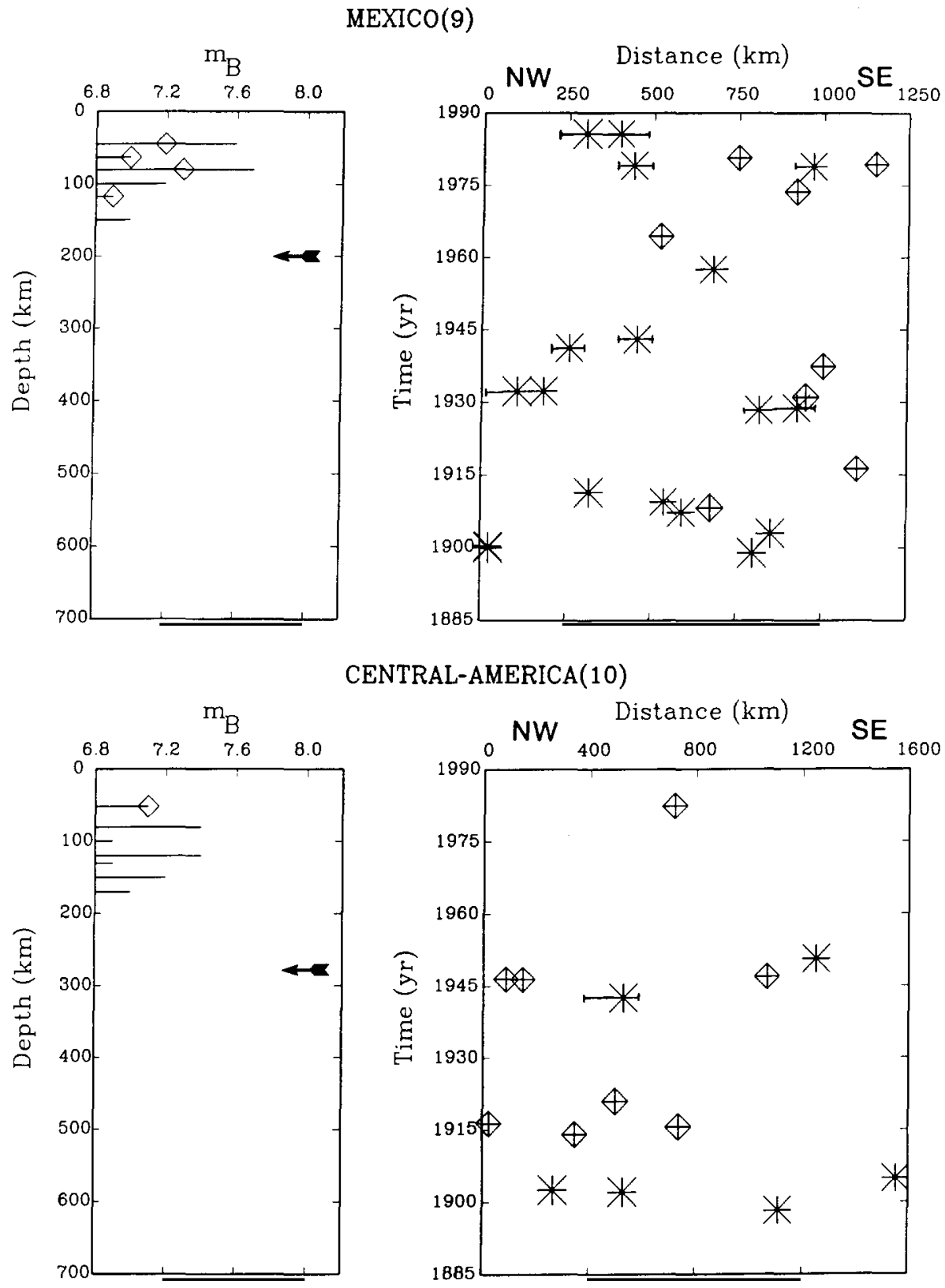

Fig. A1-5. 
143

GREATER-ANTILLES(11)

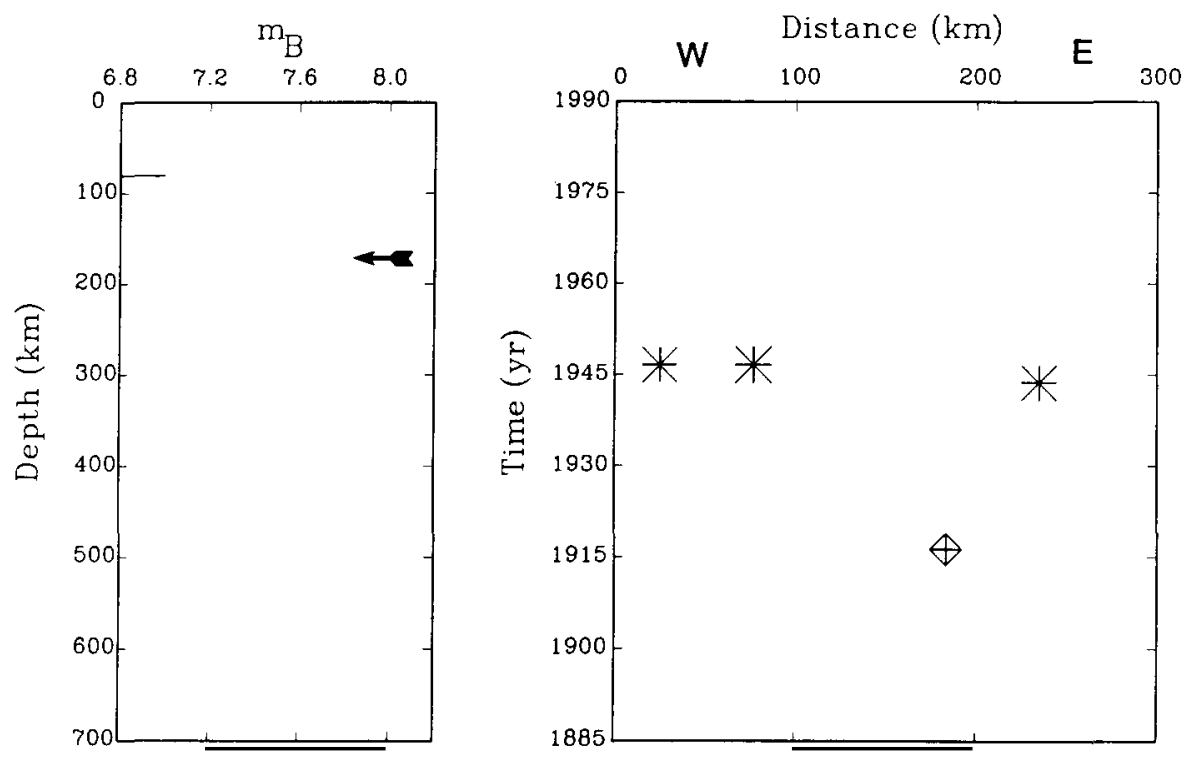

LESSER-ANTILLES(12)

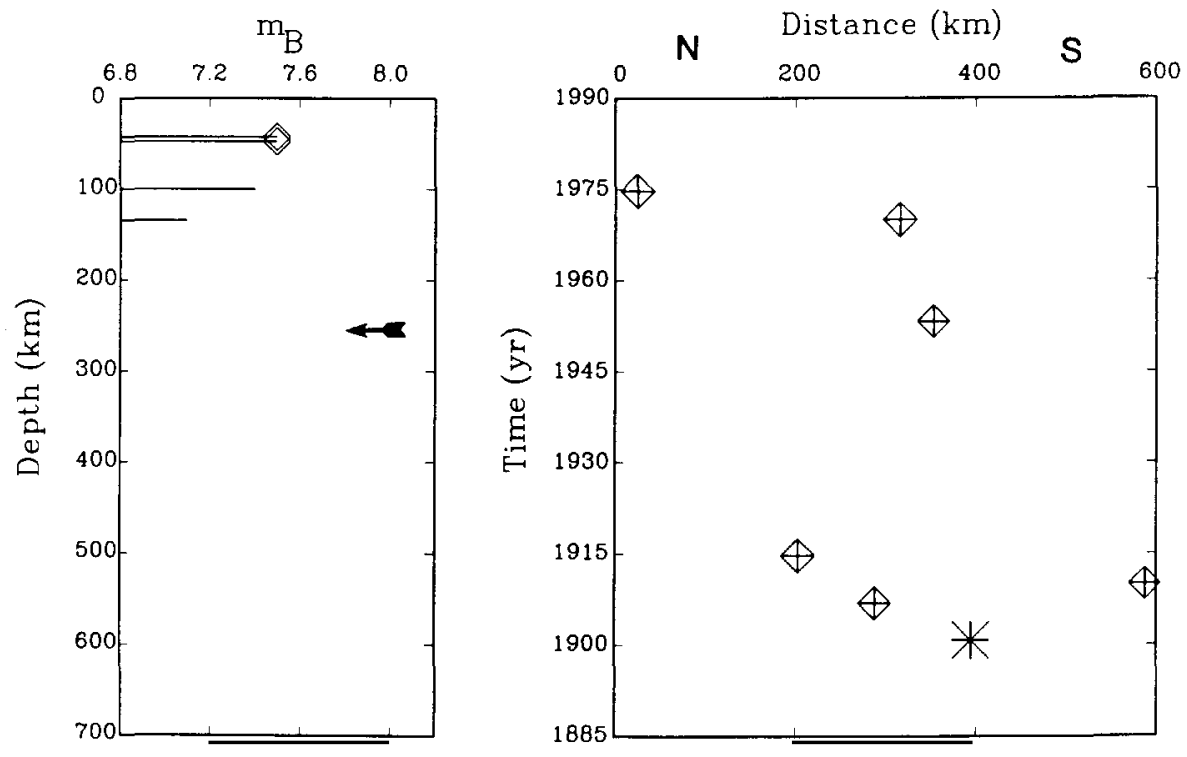

Fig. A1-6. 

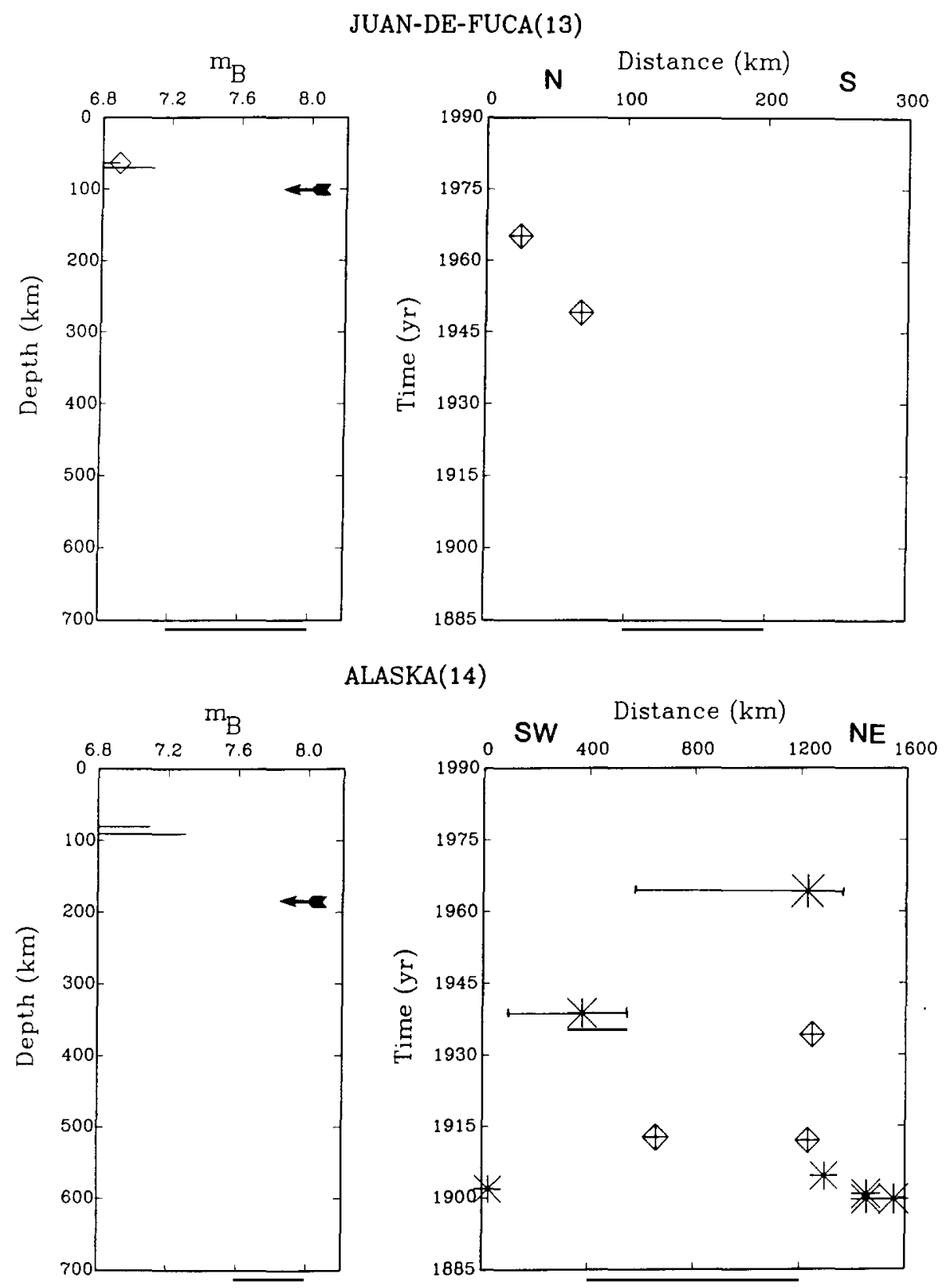

Fig. A1-7. 
145

ALEUTIANS(15)

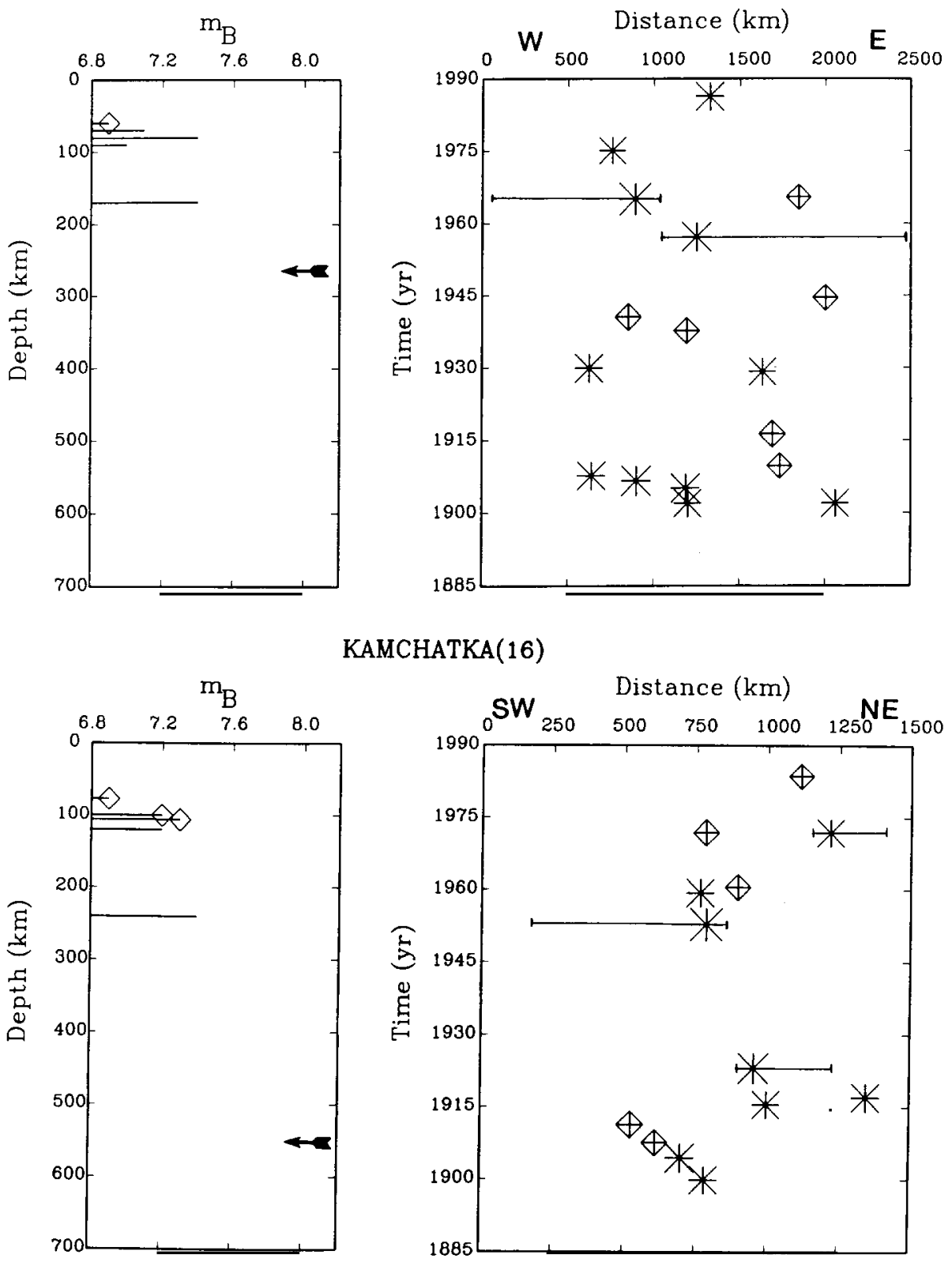

Fig. A1-8. 


\section{KURILES(17)}
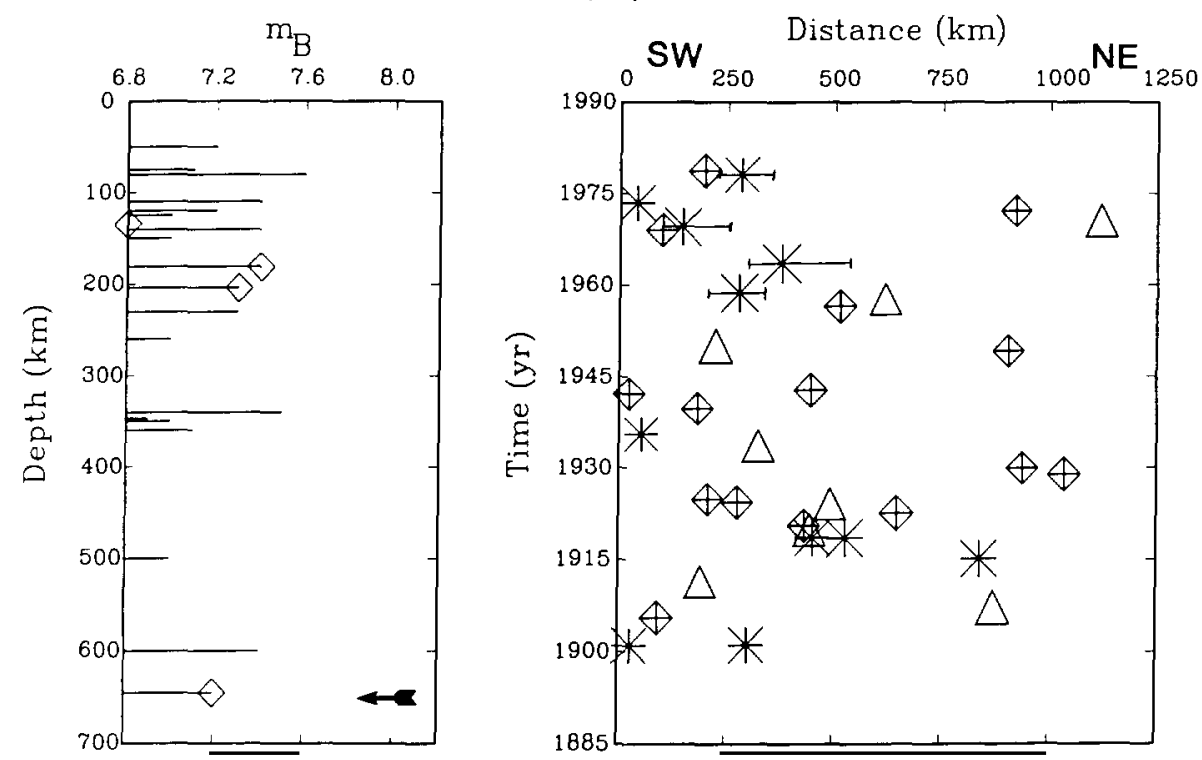

\section{NORTHEAST-JAPAN(18)}
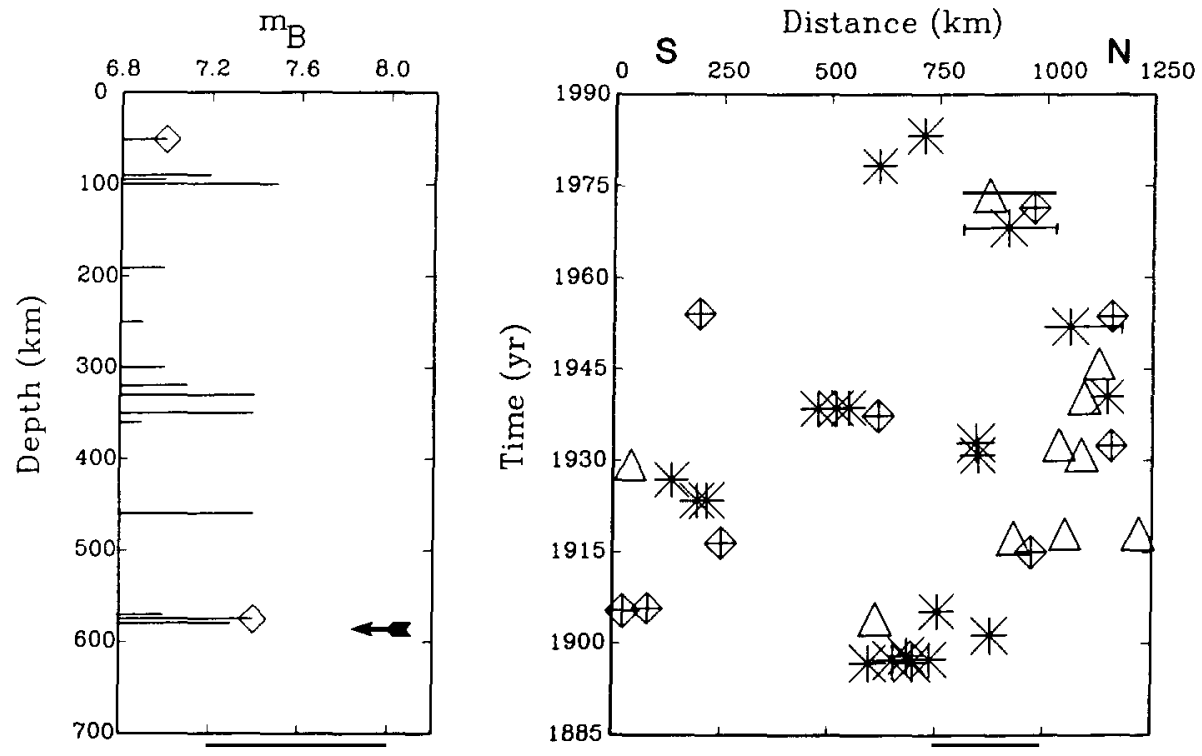

Fig. A1-9. 

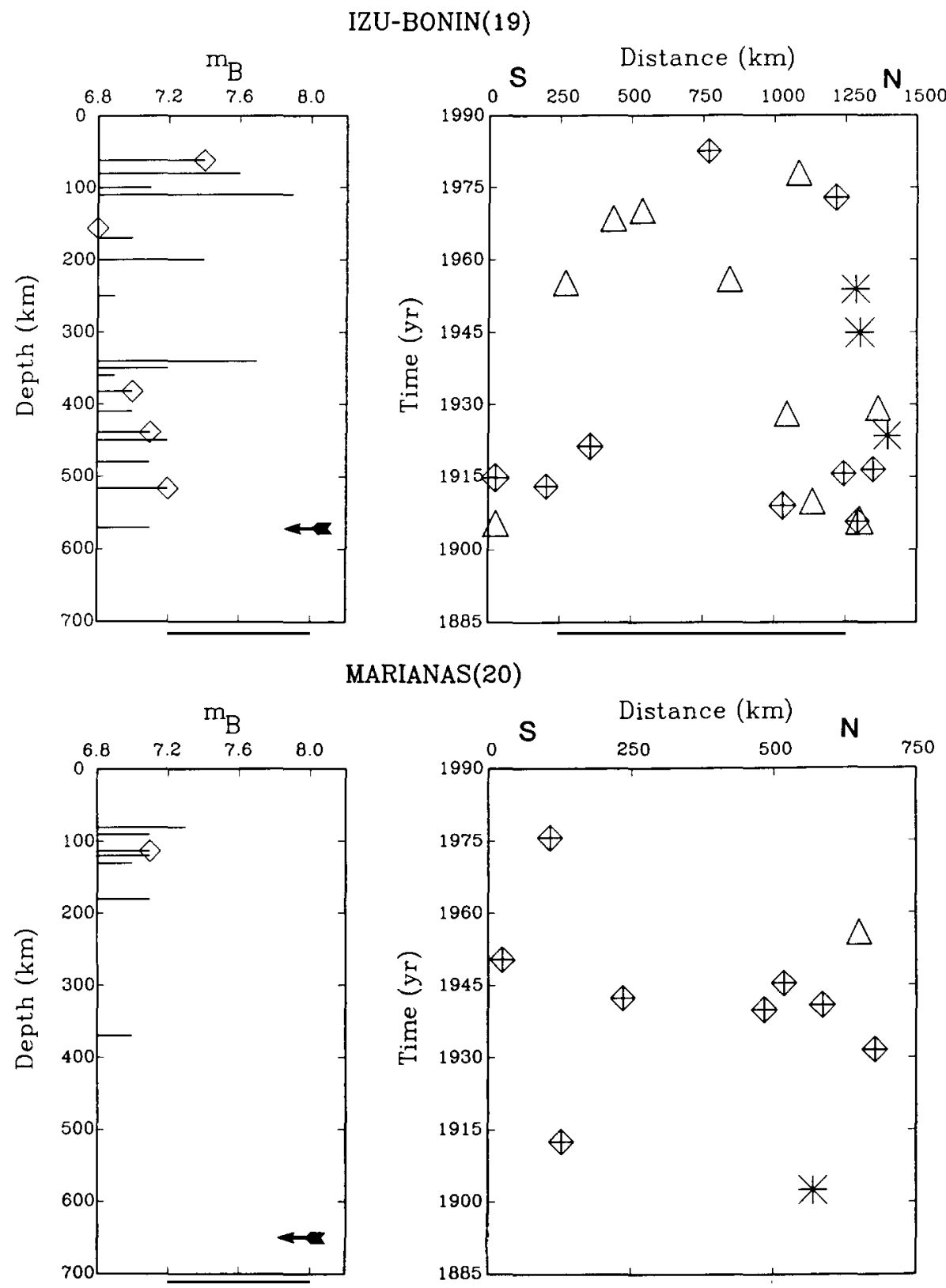

Fig. A1-10. 

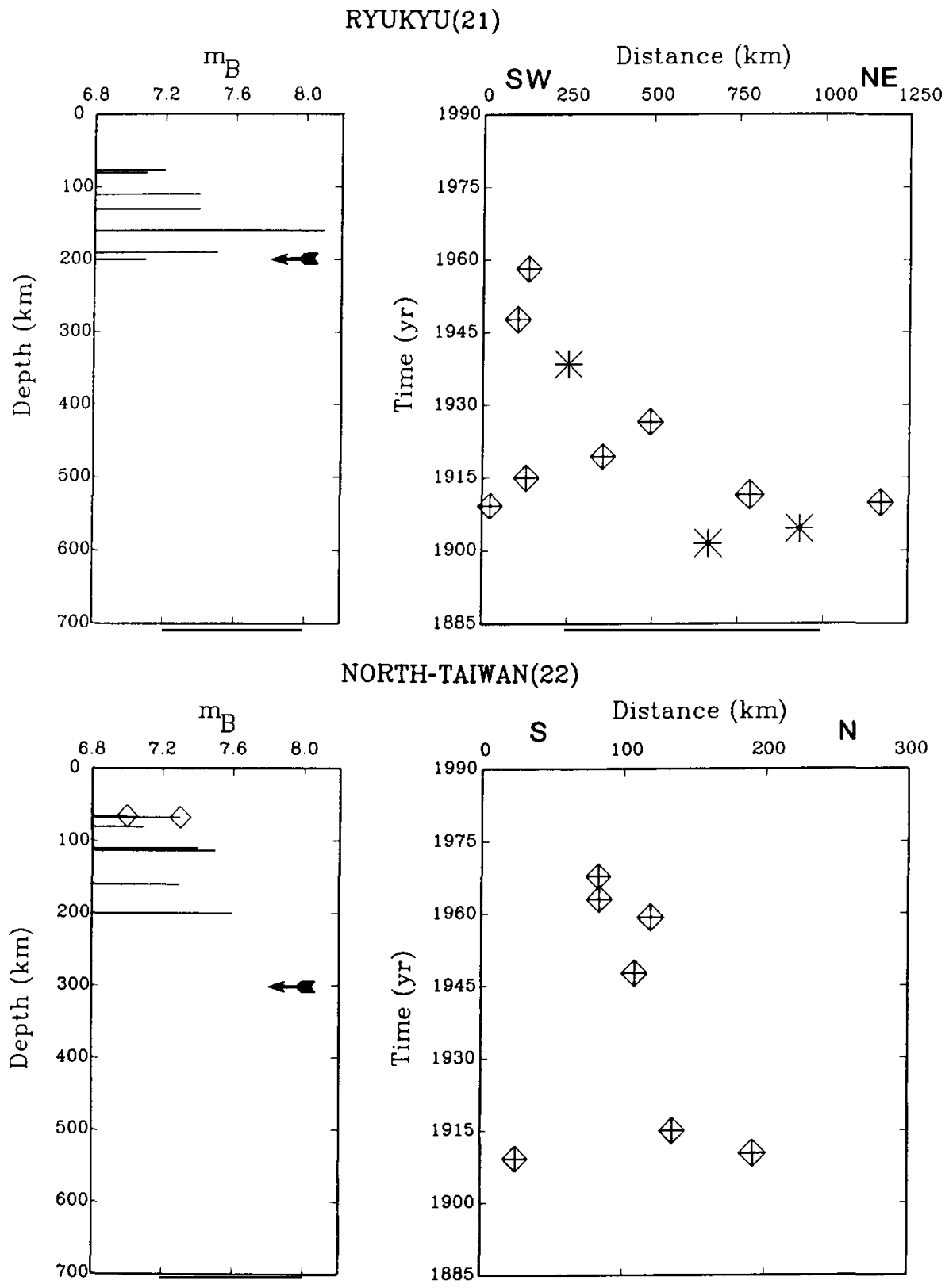

Fig. A1-11. 

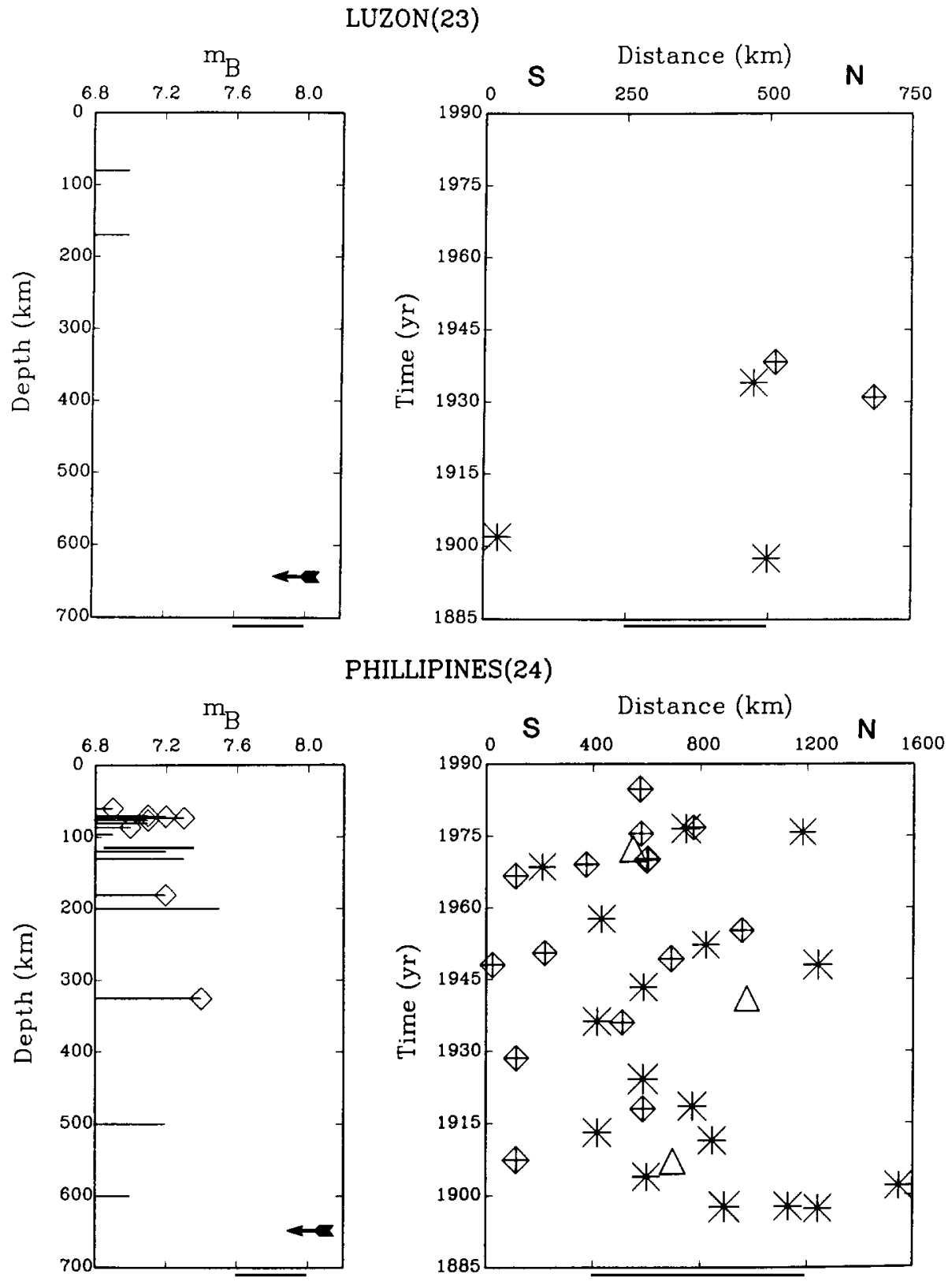

Fig. A1-12. 

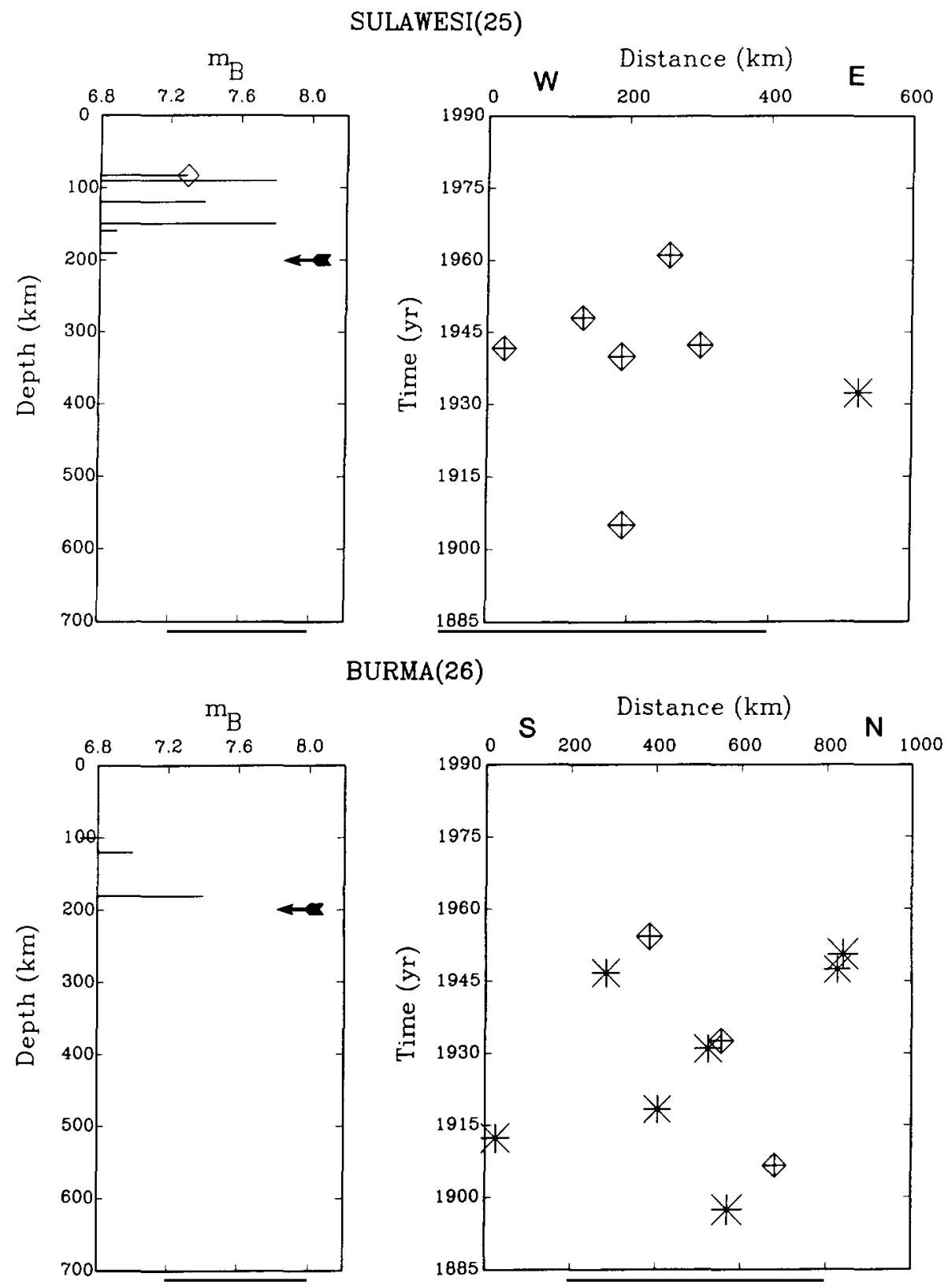

Fig. Al-13. 
151

ANDAMAN(27)

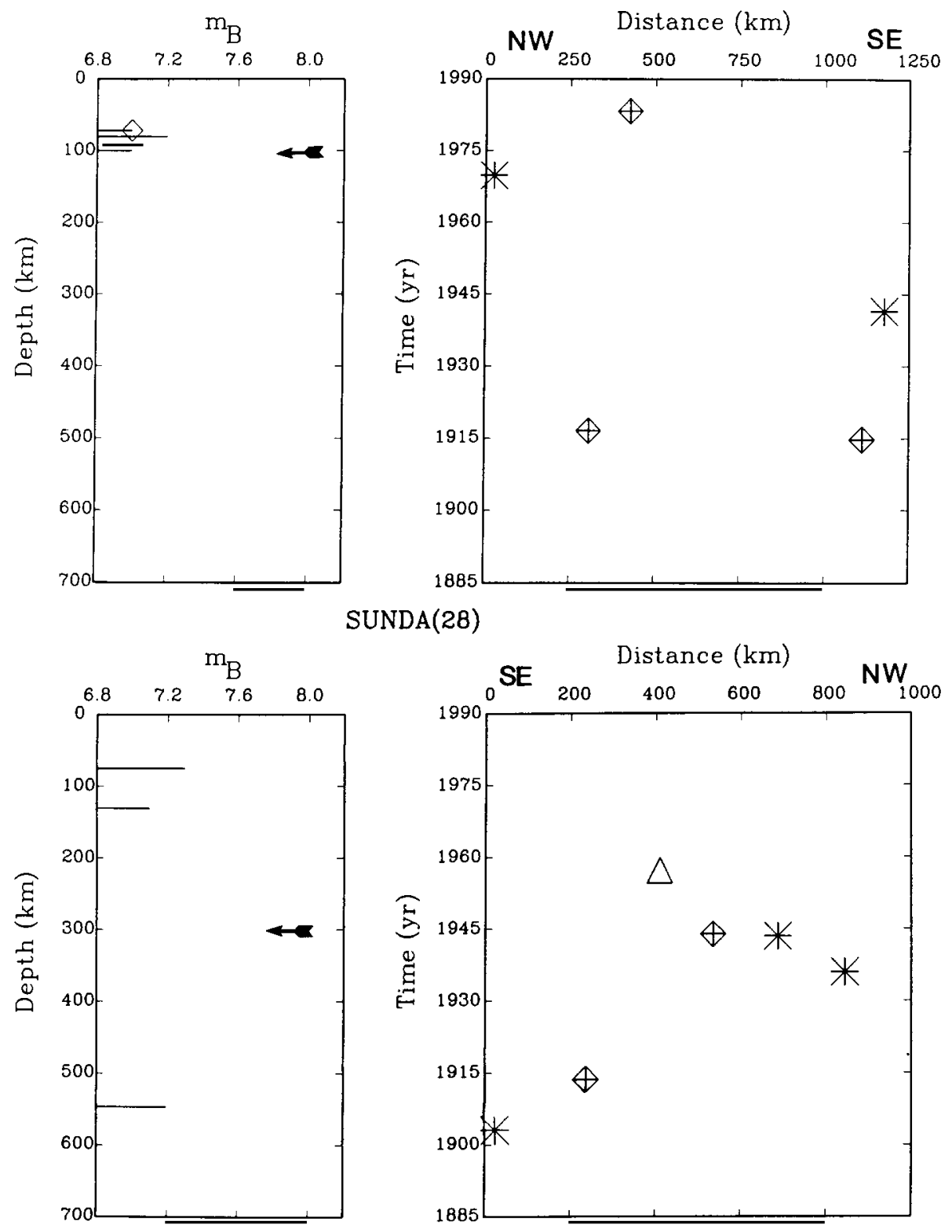

Fig. A1-14. 
JAVA(29)
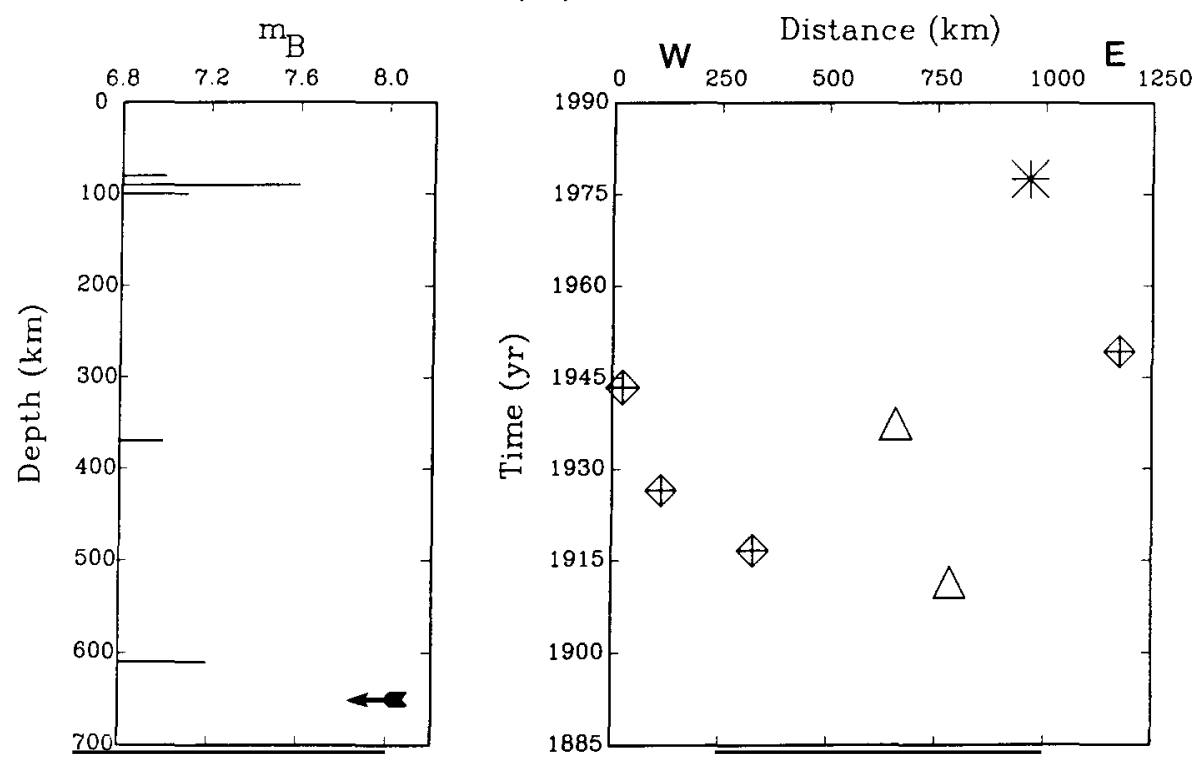

TIMOR(30)
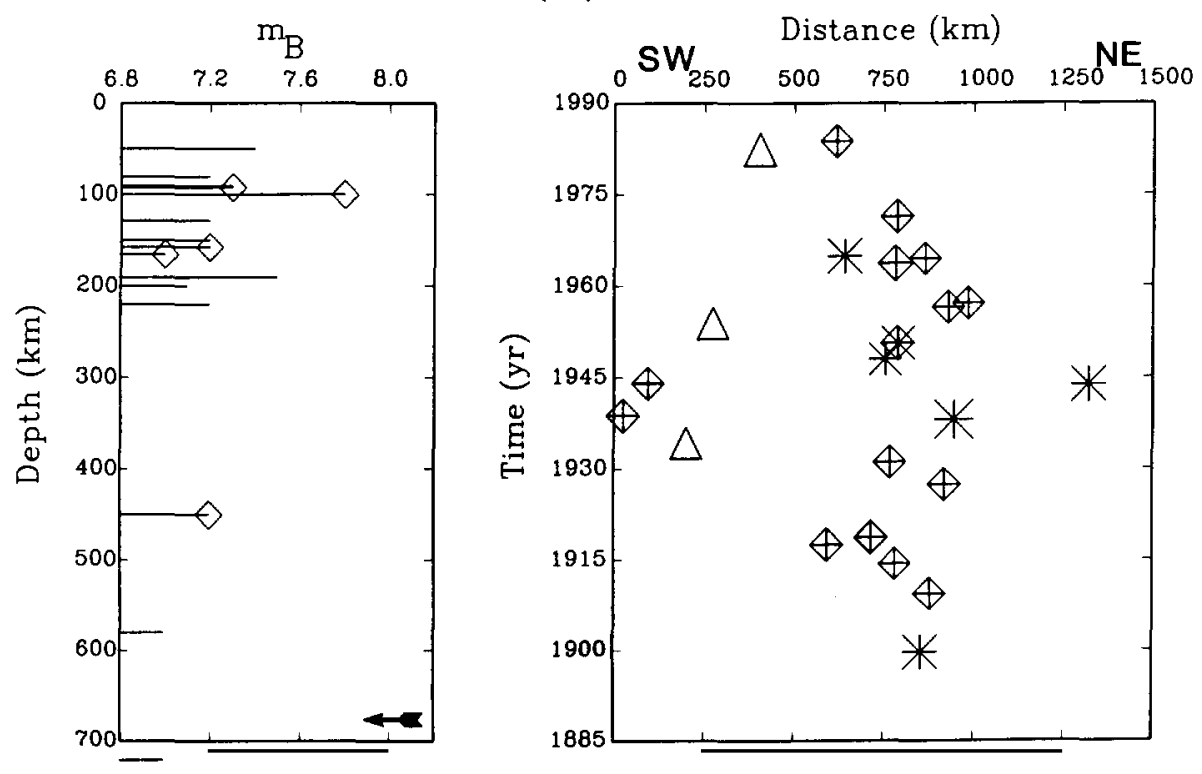

Fig. A1-15. 


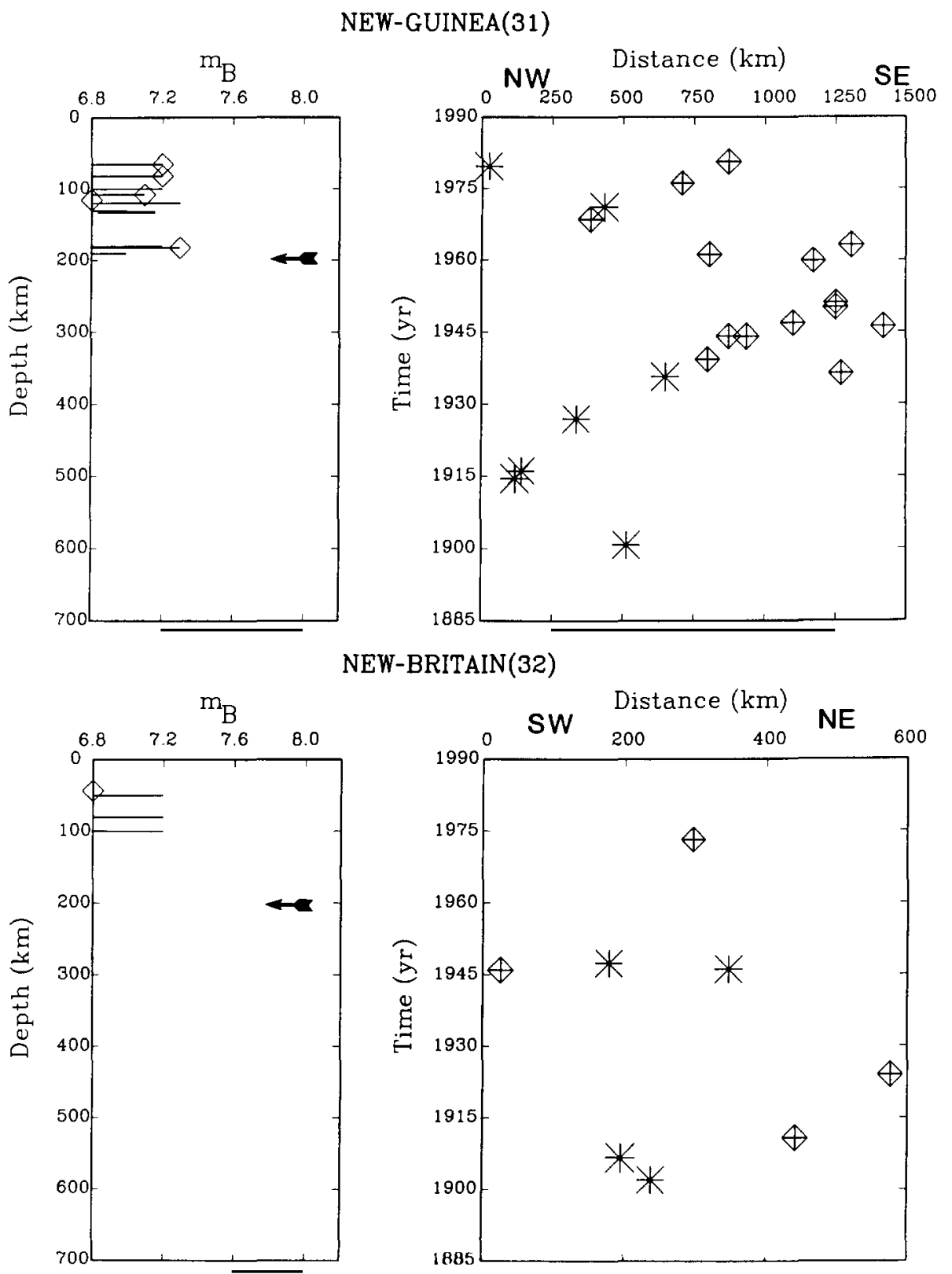

Fig. A1-16. 
154

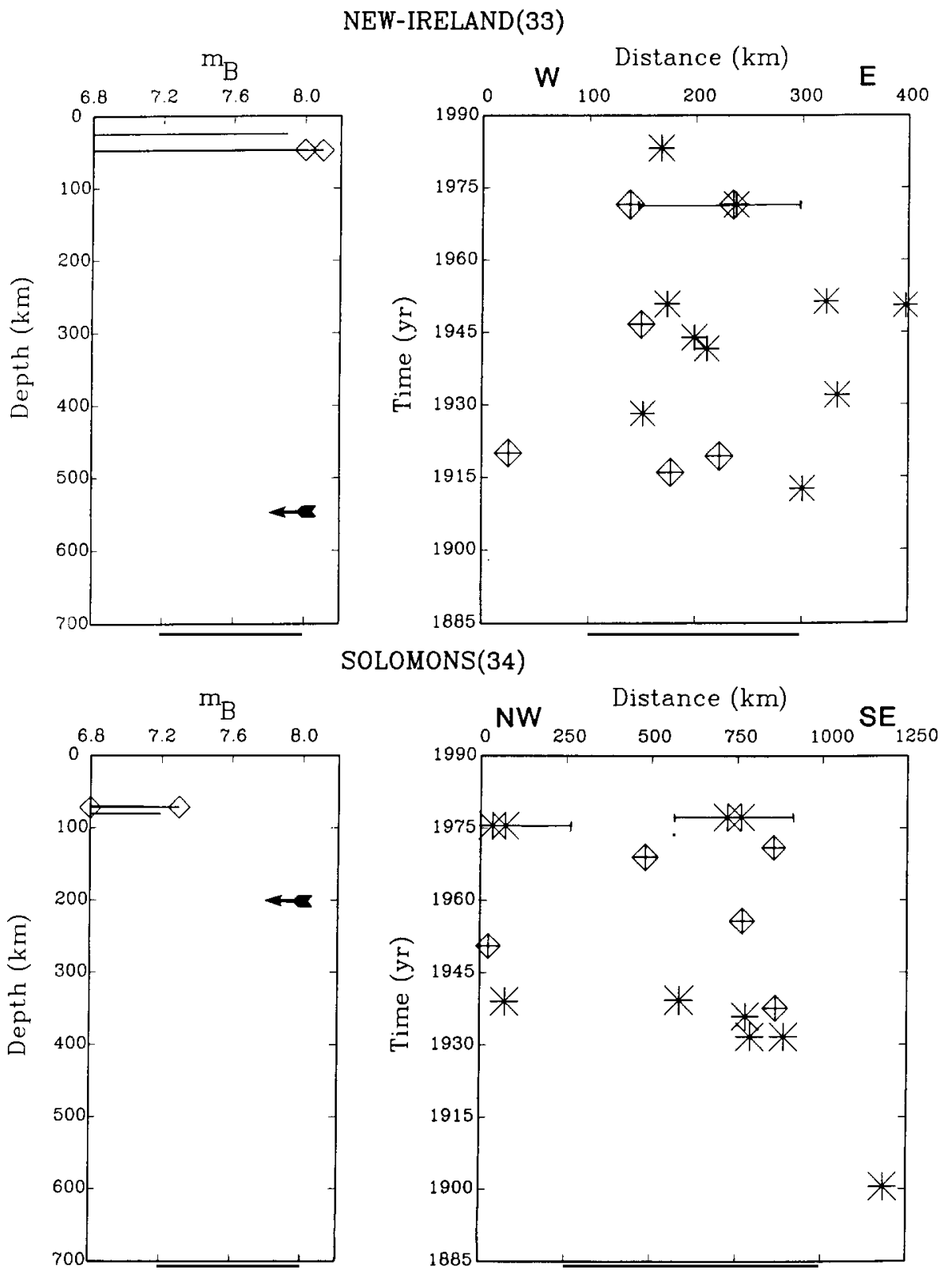

Fig. A1-17. 
155

NEW-HEBRIDES(35)
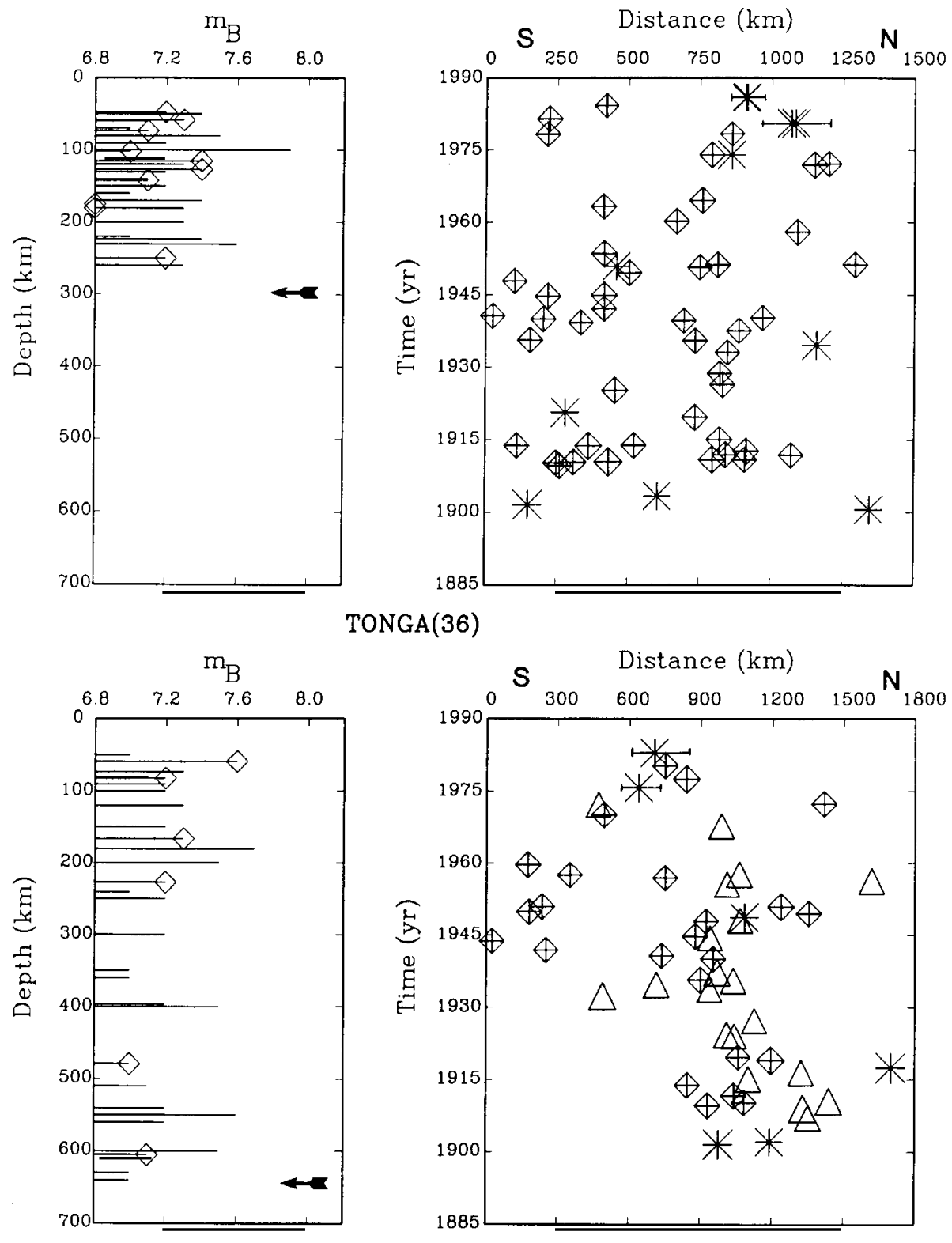

Fig. Al-18. 
156

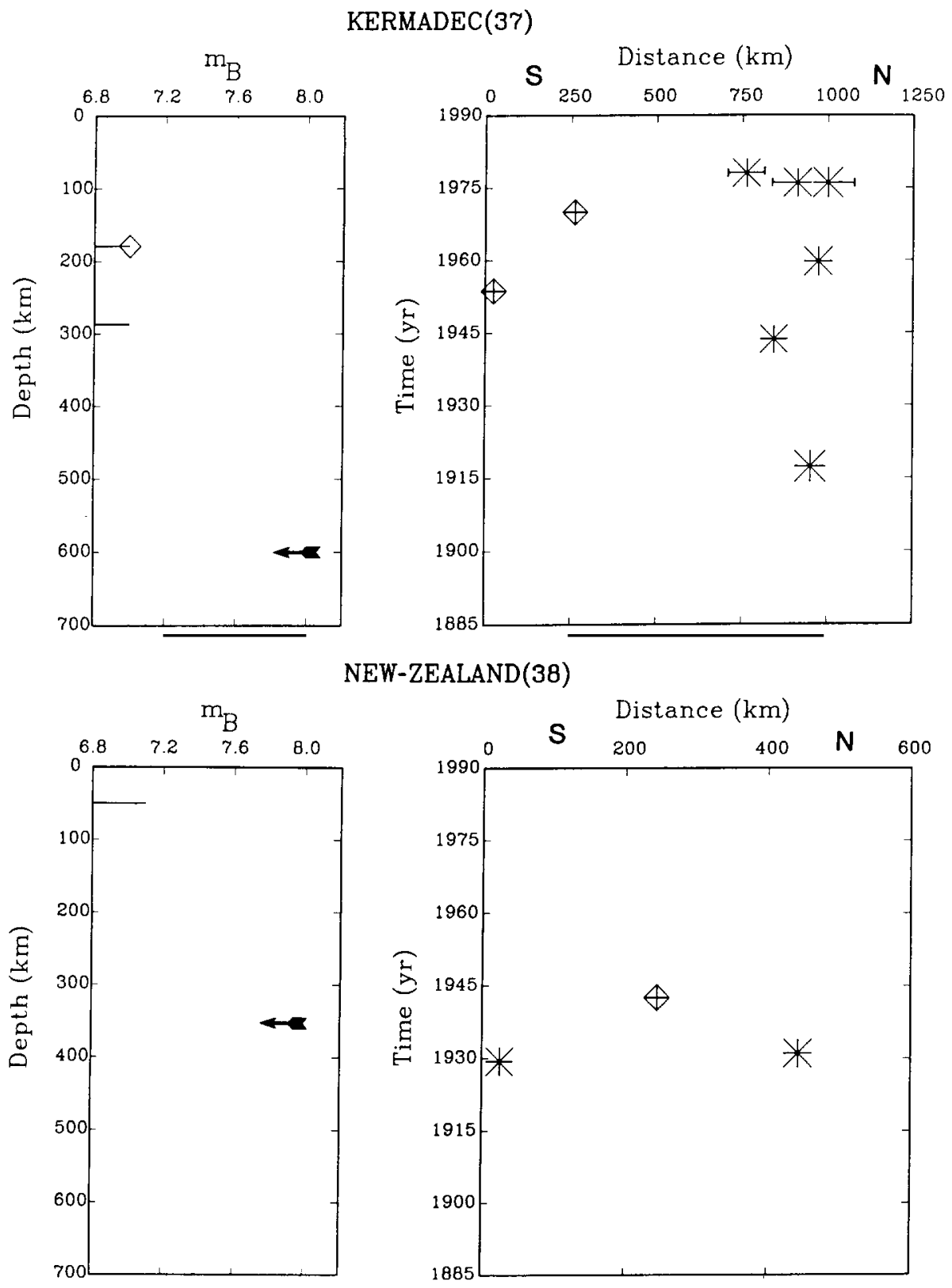

Fig. A1-19. 
157

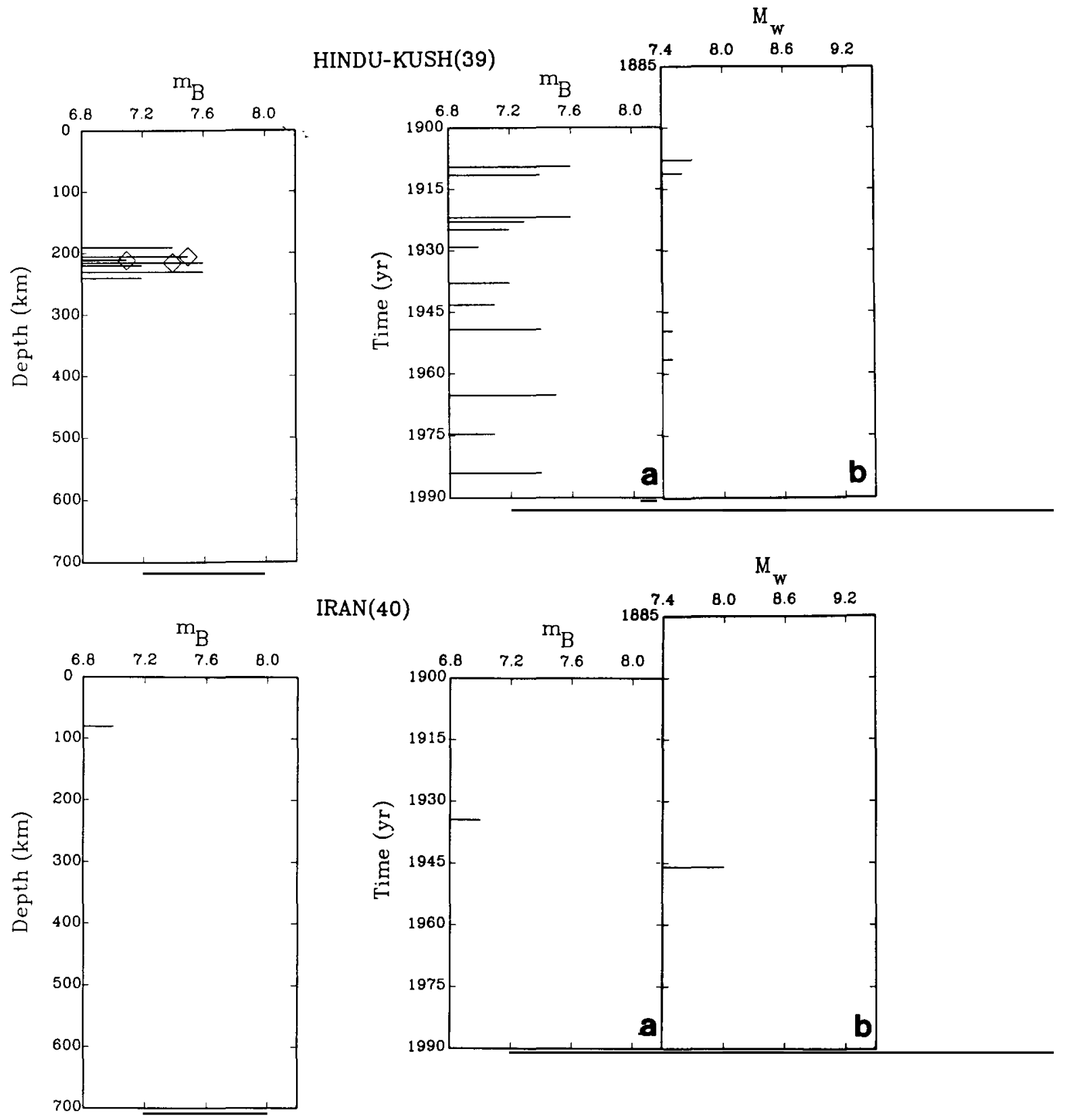

Fig. A1-20. 
158

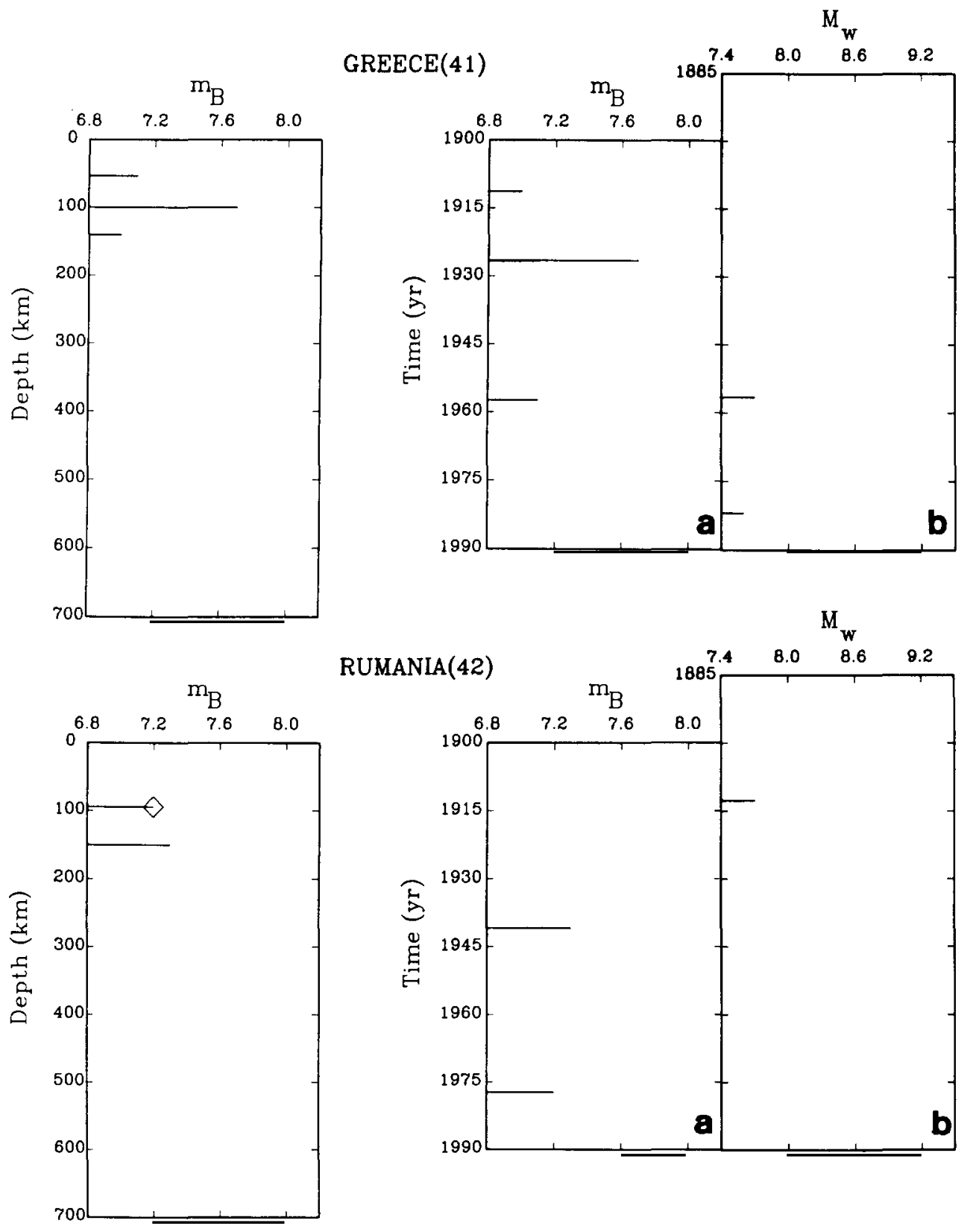

Fig. A1-21. 
159

SPAIN(43)
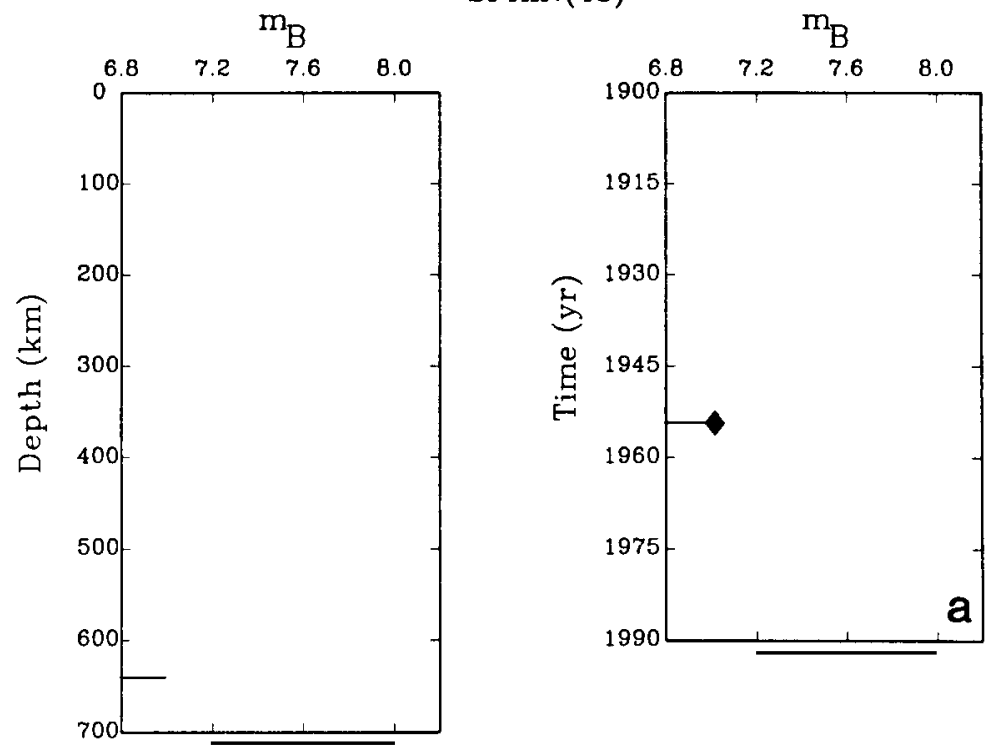

RIVERA(9')

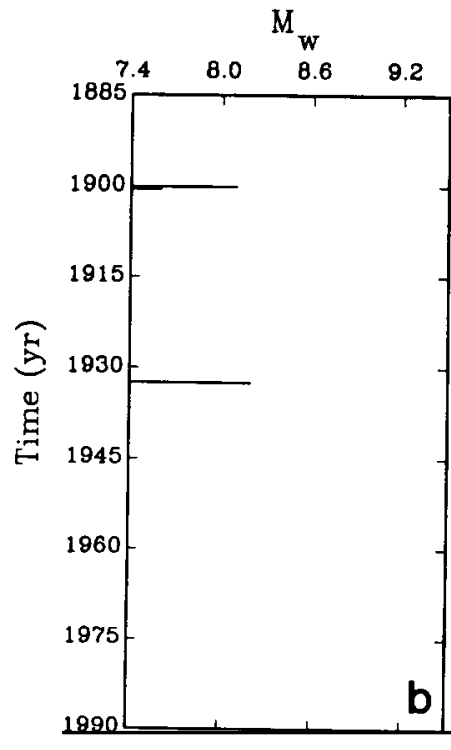

NANKAI(21')

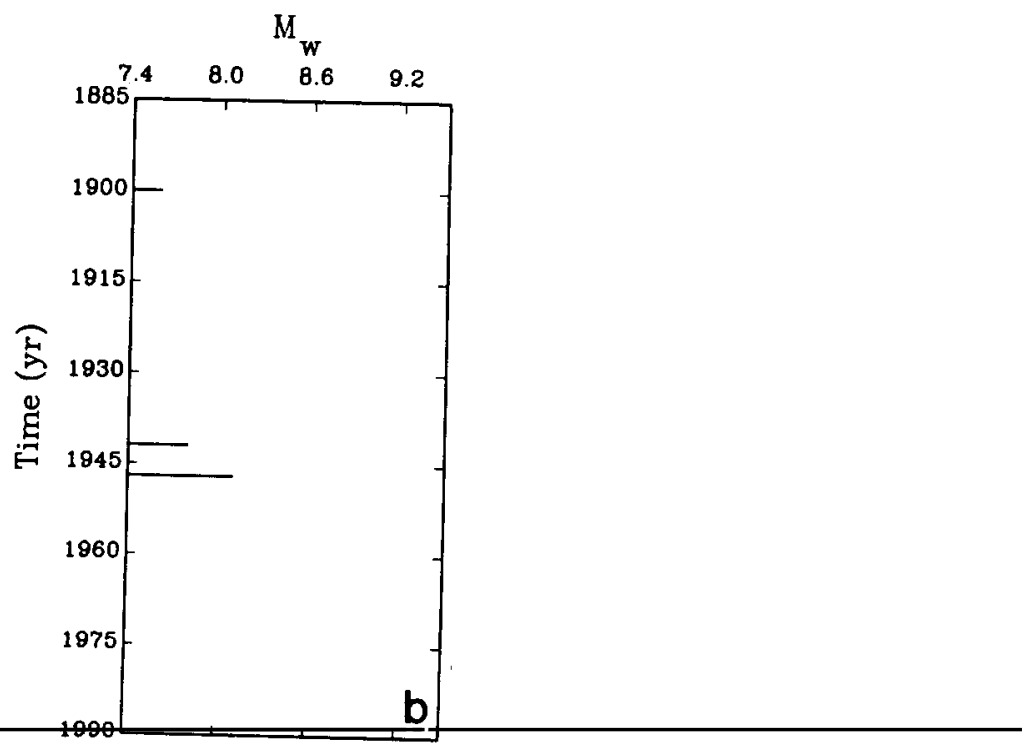

Fig. A1-22. 


\section{Appendix 2}

New focal mechanisms of intermediate-depth earthquakes were determined from first-motion data of long and short-period WWSSN records.
The mechanism diagrams in Figs. A2.1 and A2.2 are shown on an equal angle projection of the lower focal hemisphere. Closed circles are compressions and open circles, dilatations. Numbers correspond to events in Table A. The asterisk next

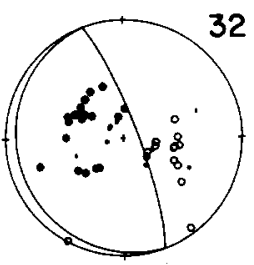

JAN, 28,1964

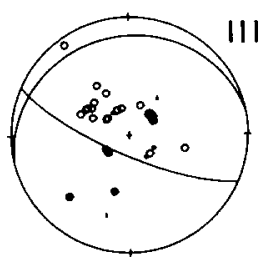

JAN. 5, $1969^{*}$

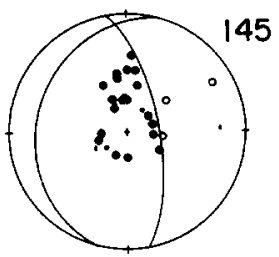

MAY $8,1971^{*}$

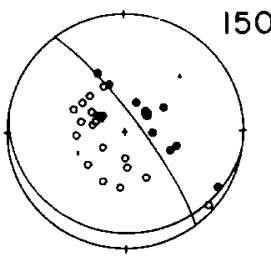

JULY 19, 197।

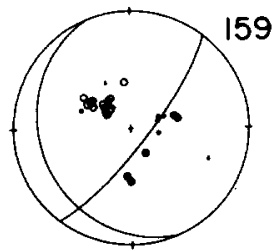

JAN. 8, $1972^{*}$
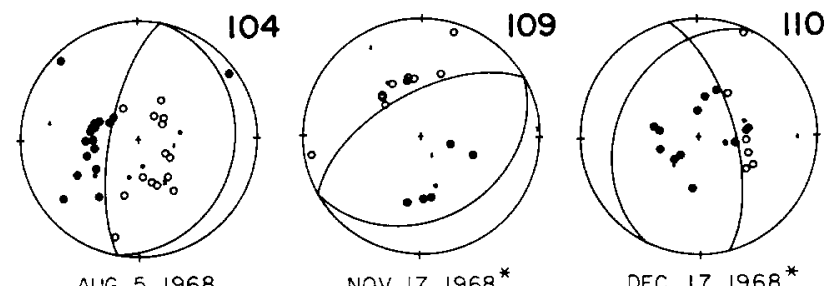

NOV. $17,1968^{*}$

DEC. $17,1968^{*}$
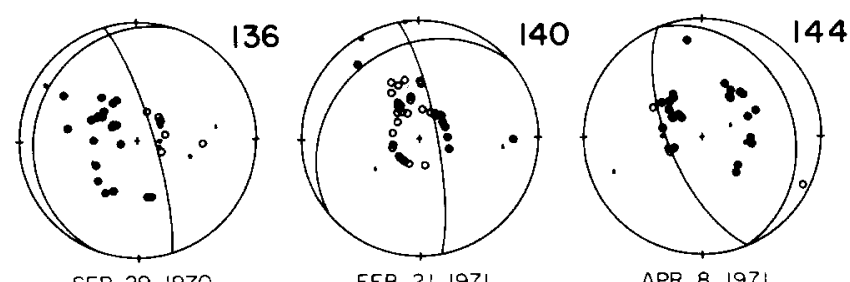

SEP. 29, 1970

FEB. $21,19 ? 1$
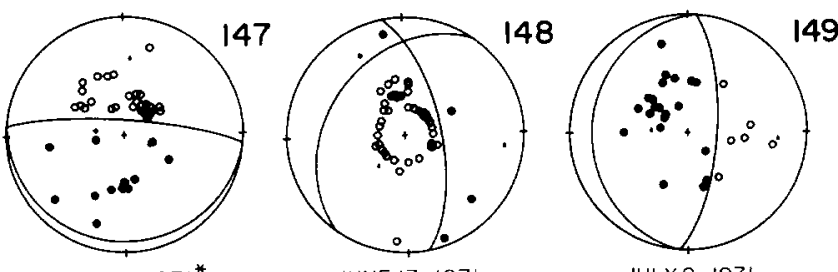

JUNE 17, 1971

JULY 8, 1971
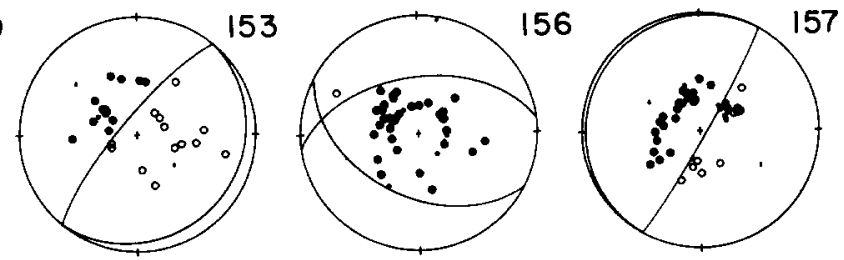

SEP. $16,1971^{*}$

NOV. $21,1971^{*}$

NOV 24,1971

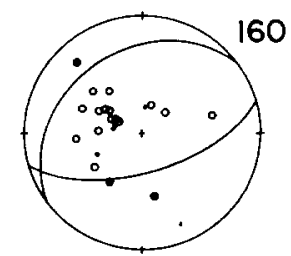

JAN. $28,1972^{*}$

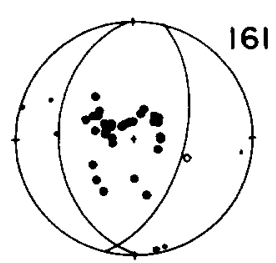

FEB. 14, 1972*

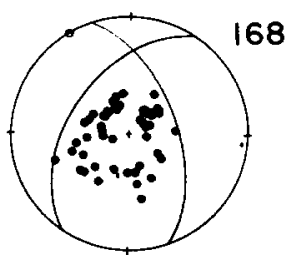

DEC 4,1972 *

Fig. A2.1. New focal mechanisms for intermediate-depth earthquakes that occurred from 1964 to 1972 . See text for symbols. 


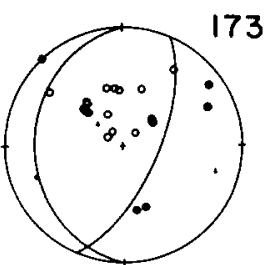

APR. 3, 1973

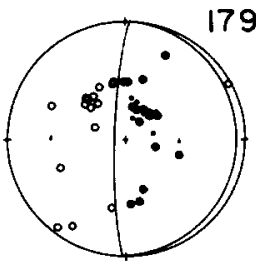

AUG 30,1973

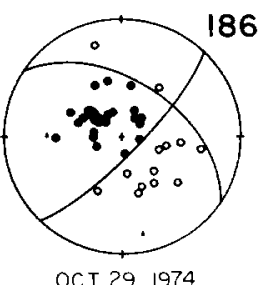

OC T. 29,1974

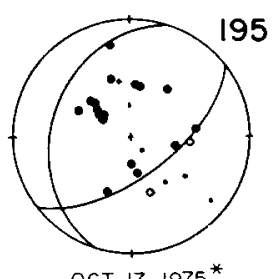

OCT. $17,1975^{*}$

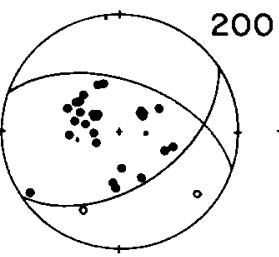

JUNE 3,1976 *

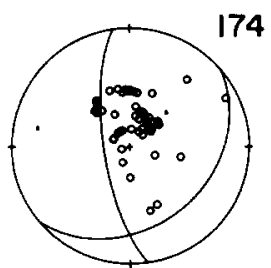

APR. 24, 1973

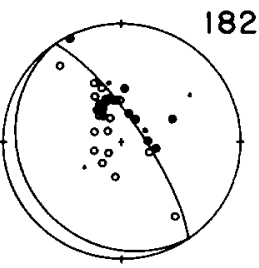

JAN. 2, 1974

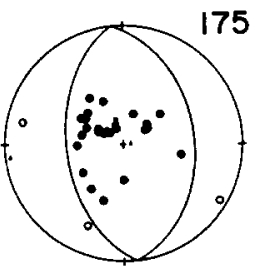

JUNE $9,1973^{*}$

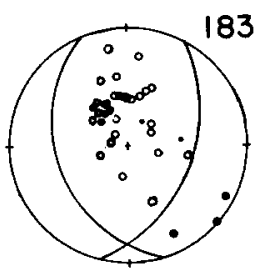

JAN. 5,1974

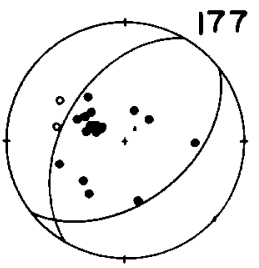

AUG. I, 1973

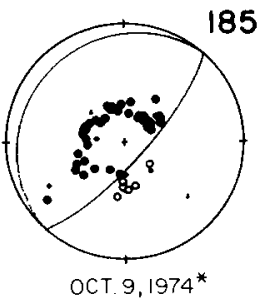

189

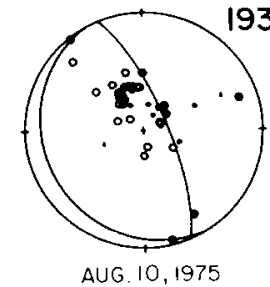

193
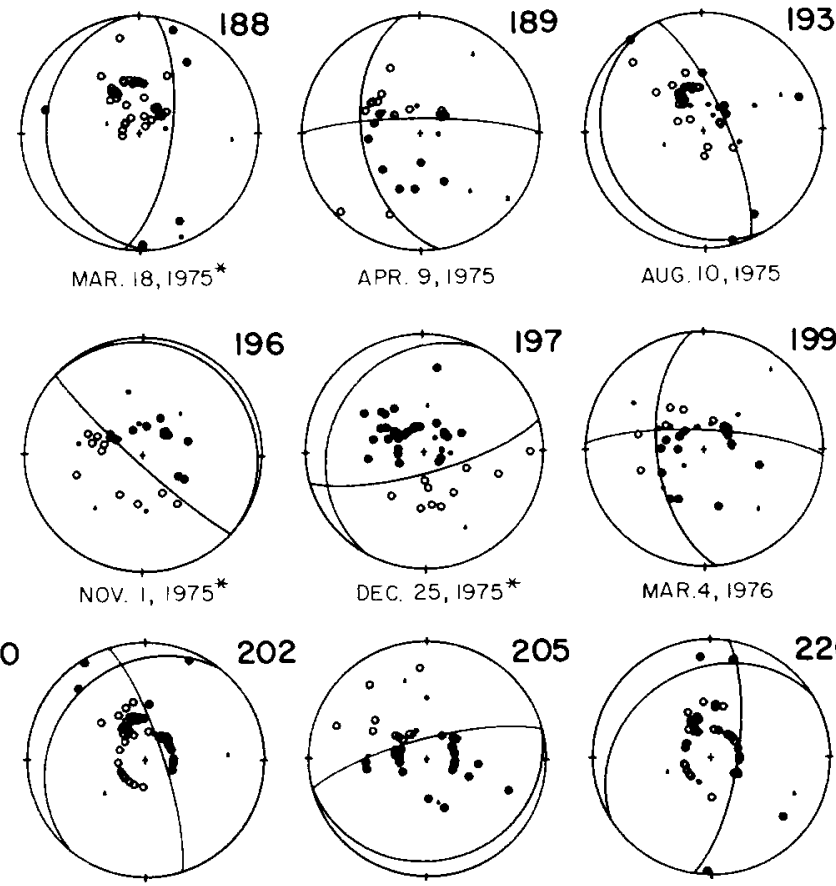

NOV. 30, 1976
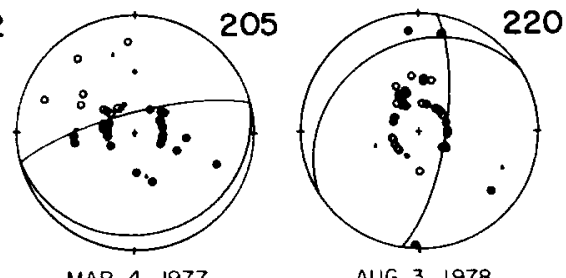

Fig. A2.2. New focal mechanism for intermediate-depth events that occurred between 1973 and 1978. See text for symbols.

to the event date indicates that one or two of the focal parameters have been constrained by modeling of a few long-period P-waveforms for these events or by timing of $(\mathrm{pP}-) \mathrm{P}$ and $(\mathrm{sp}-\mathrm{P})$ phases in short-period records to constrain the event depth. Figure A2.1 shows events that occurred from 1964 to 1972 and Figure A2.2 those events that occurred between 1973 and 1978 . 


\section{References}

Abe, K., 1972a. Focal process of the South Sandwich Island earthquake of May 26, 1964. Phys. Earth Planet. Inter., 5: $110-122$

Abe, K., 1972b. Mechanisms and tectonic implications of the 1966 and 1970 Peru earthquakes. Phys. Earth Planet. Inter., 5: $367-379$.

Abe, K., 1981. Magnitudes of large shallow earthquakes from 1904 to 1980. Phys. Earth Planet. Inter., 27: 72-92.

Abe, K., 1984. Complements to "Magnitude of large shallow earthquakes from 1904-1980". Phys. Earth Planet. Inter., 34: $17-23$.

Abe, K., 1985. Re-evaluation of the large deep earthquake of January 21, 1906. Phys. Earth Planet. Inter., 39: 157-166.

Abe, K. and Kanamori, H., 1979. Temporal variation of the activity of intermediate and deep focus earthquakes. $\mathbf{J}$. Geophys. Res., 84: 3589-3595.

Anderson, D.L., 1967. Phase changes in the upper Mantle. Science, 157: 1165-1173.

Ansell, J.H. and Smith, E.G.C., 1975. Detailed structure of a mantle seismic zone using the homogeneous station method. Nature, 253: 518-519.

Astiz, L. and Kanamori, H., 1984. An earthquake doublet in Ometepec, Guerrero, Mexico. Phys. Earth Planet. Inter., 34: 24-45.

Astiz, L. and Kanamori, H., 1986. Interplate coupling and temporal variation of mechanisms of intermediate-depth earthquakes in Chile. Bull. Seismol. Soc. Am., 76: $1614-1622$.

Banghar, A.R., 1987. Seismo-tectonics of the Andaman-Nicobar Islands. Tectonophysics, 133: 95-104.

Barazangi, M. and Isacks, B.L., 1976. Spatial distribution of earthquakes and subduction of the Nazca plate beneath South America. Geology, 4: 686-692.

Barazangi, M. and Isacks, B.L., 1979. Subduction of the Nazca plate beneath Peru; evidence from the spatial distribution of earthquakes. Geophys. J.R. Astron. Soc., 57: 537-555.

Barker, P.F., 1970. Plate tectonics of the Scotia Sea region. Nature, 228: 1293-1296.

Barker, P.F., 1972. A spreading centre in the East Scotia Sea. Earth Planet. Sci. Lett., 15: 123-132.

Beck, S.L. and Ruff, L.J., 1984. The rupture process of the great 1979 Colombia earthquake: evidence for the asperity model. J. Geophys. Res., 89: 9281-9291.

Beck, S.L. and Ruff, L.J., 1987. Source process of the 1963 Kurile Islands earthquake. J. Geophys. Res. (submitted).

Billington, S., 1980. The morphology and tectonics of the subducted lithosphere in the Tonga-Fiji-Kermadec region from seismicity and focal mechanism solutions. Ph.D. thesis, University of Cornell, $220 \mathrm{pp}$.

Burbach, G.V. and Frohlich, C., 1986. Intermediate and deep seismicity and lateral structure of subducted lithosphere in the Circum-Pacific region. Rev. Geophys., 24: 833-874.

Cardwell, R.K. and Isacks, B.L., 1978. Geometry of subducted lithosphere beneath the Banda Sea in eastern Indonesia from seismicity and fault plane solutions. J. Geophys. Res., 83: $2825-2838$.
Cardwell, R.K., Isacks, B.L. and Karig, D.E., 1980. The spatial distribution of earthquakes, focal mechanism solutions and subducted lithosphere in the Philippine and North Indonesia Island. In: D.E. Hayes (Editor), The Tectonic and Geologic Evolution of the Southeast Asian Seas and Islands. Geophys. Monogram 23, Washington, DC, pp. $1-35$.

Chase, C.G., 1978. Extension behind island arcs and motion relative to hot spots. J. Geophys. Res., 83: 5385-5387.

Chapple, W.M. and Forsyth, D.M., 1979. Earthquakes and bending of plates at trenches. J. Geophys. Res., 84: 6729-6749.

Chin, D.S. and Isacks, B.L., 1983. Accurate source depths and focal mechanisms of shallow earthquakes in western South America and in the New Hebrides Island arc. Tectonics, 2: $529-563$.

Christensen, D. and Ruff, L.J., 1983. Outer-rise earthquakes and seismic coupling. Geophys. Res. Lett., 10: 697-700.

Christensen, D. and Ruff, L.J., 1987. Outer-rise earthquakes and their relationship to seismic coupling (preprint).

Chung, W.Y. and Kanamori, H., 1976. Source process and tectonic implications of the Spanish deep-focus earthquake of March 29, 1954. Phys. Earth Planet. Inter., 13: 85-96.

Chung, W.Y. and Kanamori, H., 1978. Subduction process of a fracture zone and aseismic ridges. The focal mechanism and source characteristics of the New Hebrides earthquake of 1969 , January 19 and some related events. Geophys. J. R. Astron. Soc., 54: 221-240.

Chung, W.Y. and Kanamori, H., 1980. Variation of seismic source parameters and stress drop within a descending slab and its implications in plate mechanics. Phys. Earth Planet. Inter., 23: 134-159.

Clowes, R.M., Ellis, R.M., Jajnal, Z. and Jones, I.F., 1983. Seismic reflections from subducting lithosphere? Nature, 303: 668-670.

Crosson, R.S., 1980. Review of seismicity in the Puget Sound region from 1970 to 1978 . In: Proceedings of Workshop XIV, Earthquake Hazards of the Puget Sound Region, Washington. U.S. Geol. Surv., Open-File Report 83-19, pp. 6-18.

Dean, B.W. and Drake, C.L., 1978. Focal mechanism solutions and tectonics of the Middle America arc. J. Geology, 86: 111-128.

Denham, D., 1977. Summary of earthquake focal mechanisms for the western Pacific-Indonesia region 1929-1973. NOAA, U.S. Dept. of Commerce Report SE-3.

Dewey, J.W. and Algermissen, S.T., 1974. Seismicity of the Middle America arc-trench systems near Managua, Nicaragua. Bull. Seismol. Soc. Am., 64: 1033-1048.

Dewey, J.W. and Spence, W., 1979. Seismic gaps and source zones of recent large earthquakes in coastal Peru. Pageoph, 117: 1148-1171.

Dmowoska, R., Rice, J.R., Lovison, L.C. and Josell, D., 1987 Stress transfer and phenomena in coupled subduction zones during the earthquake cycle (preprint).

Dziewonski, A.M. and Woodhouse, J.H., 1983. An experiment in the systematic study of global seismicity; centroid-moment tensor solutions for 201 moderate and large earthquakes of 1981. J. Geophys. Res., 88: 3247-3271. 
Dziewonski, A.M., Friedman, A., Giardini, D. and Woodhouse, J.H., 1983a. Global seismicity of 1982: centroid-moment tensor solutions for 308 earthquakes. Phys. Earth Planet. Inter., 33: 76-90.

Dziewonski, A.M., Friedman, A. and Woodhouse, J.H., 1983b. Centroid-moment tensor solutions for January-March 1983. Phys. Earth Planet. Inter., 33: 71-75.

Dziewonski, A.M., Franzen, J.E. and Woodhouse, J.H., 1983c. Centroid-moment tensor solutions for April-June 1983. Phys. Earth Planet. Inter., 33: 243-249.

Dziewonski, A.M., Franzen, J.E. and Woodhouse, J.H., 1984a. Centroid-moment tensor solutions for July-September 1983. Phys. Earth Planet. Inter., 34: 1-8.

Dziewonski, A.M., Franzen, J.E. and Woodhouse, J.H., 1984b. Centroid-moment tensor solutions for October-December 1983. Phys. Earth Planet. Inter., 34: 129-136.

Dziewonski, A.M., Franzen, J.E. and Woodhouse, J.H., 1984c. Centroid-moment tensor solutions for January-March 1984. Phys. Earth Planet. Inter., 34: 209-219.

Dziewonski, A.M., Franzen, J.E. and Woodhouse, J.H., 1985a. Centroid-moment tensor solutions for April-June 1984. Phys. Earth Planet. Inter., 37: 87-96.

Dziewonski, A.M., Franzen, J.E. and Woodhouse, J.H., 1985b. Centroid-moment tensor solutions for July-September 1984. Phys. Earth Planet. Inter., 38: 203-213.

Earthquake Data Report, U.S.G.S., 1984.

Eguchi, T., Uyeda, S. and Maki, T., 1979. Seismotectonics and tectonic history of the Andaman Sea. Tectonophysics, 57: $35-51$.

Engdahl, E.R., 1977. Seismicity and plate subduction in the Central Aleutians. In: M. Talwani and W.C. Pitman III (Editors), Island Arcs, Deep Sea Trenches and Backarc Basins. Am. Geophysical Union, Washington, DC.

Engdahl, E.R. and Scholz, C.H., 1977. A double Benioff zone beneath the central Aleutians: An unbending of the lithosphere. Geophys. Res. Lett., 4: 473-476.

Engdahl, E.R., Sleep, N.H. and Lin, M.T., 1977. Plate effects in North Pacific subduction zones. Tectonophysics, 37: $95-116$.

Fedotov, S.A., 1968. On deep structure, properties of the upper mantle, and volcanism of the Kuril-Kamchatka island arc according to seismic data. Am. Geophys. Union, Geophys. Monogram, 12: 131-139.

Fedotov, S.A., Bagdasarova, S.M., Kuzin, I.P. and Tarakanov, R.Z., 1971. Earthquakes and Deep Structure of the South Kurile Island Arc. Israel Program for Scientific Translations, Jerusalem, vi $+249 \mathrm{pp}$.

Fitch, T.J., 1970a. Earthquake mechanisms and island arc tectonics in the Indonesian-Philippine region. Bull. Seismol. Soc. Am., 60: 565-591.

Fitch, T.J., 1970b. Earthquake mechanisms in the Himalayan, Burmese, and Andaman regions and continental tectonics in central Asia. J. Geophys. Res., 75: 2699-2709.

Fitch, T.J., 1972. Plate convergence, transcurrent faults and internal deformation adjacent to southeast Asia and the western Pacific. J. Geophys. Res., 77: 4432-4460.
Fitch, T.J. and Molnar, P., 1970. Focal mechanisms along inclined earthquake zones in the Indonesia-Philippine region. J. Geophys. Res., 75: 1431-1444.

Forsyth, D.W., 1975. Fault plane solutions and tectonics of the south Atlantic and Scotia Sea. J. Geophys. Res., 80: 1429-1443.

Frankel, A., 1982. A composite focal mechanism for microearthquakes along the northeastern border of the Caribbean plate. Geophys. Res. Lett., 9: 511-514.

Frankel, A. and McCann, W.R., 1979. Moderate and large earthquakes in the South Sandwich arc: Indications of tectonic variation along a subduction zone. J. Geophys. Res., 84: 5571-5577.

Frankel, A., McCann, W.R. and Murphy, A.J., 1980. Observations from a seismic network in the Virgin Island region: Tectonic structures and earthquake swarms. J. Geophys. Res., 85: 2669-2678.

Fujita, K. and Kanamori, H., 1981. Double seismic zones and stresses of intermediate depth earthquakes. Geophys. J. R. Astron. Soc., 66: 131-156.

Fukao, Y., Hori, S. and Ukawa, M., 1983. A seismological constraint on the depth of basalt-eclogite transition in a subducting oceanic crust. Nature, 303: 413-415.

Giardini, D., 1984. Systematic analysis of deep seismicity: 200 centroid moment tensor solutions for earthquakes between 1977 and 1980. Geophys. J. R. Astron. Soc., 77: 883-914.

Giardini, D., 1987. Quantification and frequency distribution of deep earthquakes. J. Geophys. Res. (submitted).

Giardini, D. and Woodhouse, J.H., 1984. Deep seismicity and modes of deformation in Tonga subduction zone. Nature, 307: 505-509.

Giardini, D., Dziewonski, A.M. and Woodhouse, J.H., 1985. Centroid-moment tensor solutions for 113 large earthquakes in 1977-1980. Phys. Earth Planet. Inter., 40: 259-272.

Given, J.W. and Kanamori, H., 1980. The depth extent of the 1977 Sumbawa, Indonesia earthquake. EOS, 61: 1044.

González, J., McNally, K. and Rial, J., 1984. Recent normal faulting in southcentral Mexico: A study of source processes and regional tectonic implications (preprint).

González-Ruiz, J.R., 1986. Earthquake source mechanics and tectonophysics of the Middle America subduction zone in Mexico. Ph. D. thesis, University of California, Santa Cruz.

Goto, K., Hamaguchi, H. and Suzuki, Z., 1985. Earthquake generating stresses in a descending slab. Tectonophysics, 112: $111-128$

Gutenberg, B. and Richter, C.F., 1954. Seismicity of the Earth, 2nd Edn. Princeton University Press, Princeton, NJ, 330 pp.

Hasegawa, A. and Sacks, S., 1981. Subduction of the Nazca plate beneath Peru as determined from seismic observations. J. Geophys. Res., 86: 4971-4980.

Hasegawa, A. and Umino, N., 1978. Focal mechanisms and the distribution of seismicity in northeast Japan. Progr. Abstr. Seismol. Soc. Jpn., 1: 34 (in Japanese).

Hasegawa, A., Umino, N., Takagi, A. and Suzuki, Z., 1979. 
Double-planed deep seismic zone and anomalous structure in the upper mantle beneath northeastern Honshu (Japan). Tectonophysics, 57: 1-6.

Heaton, T.H. and Kanamori, H., 1984. Seismic potential associated with subduction in the Northwestern United States. Bull. Seismol. Soc. Am., 74: 933-941.

Hodgson, J.H. and Cock, J.I., 1956. Tellus, 8: 321-328.

House, L.S. and Jacob, K.H., 1982. Thermal stresses in subducting lithosphere can explain double seismic zones. Nature, 295: 587-589.

House, L.S. and Jacob, K.H., 1983. Earthquakes, plate subduction and stress reversals in the eastern Aleutian arc. J. Geophys. Res., 88: 9347-9373.

Hudnut, K.W. and Taber, J.J., 1987. Transition from double to single Wadati-Benioff seismic zone in the Shumagin Islands, Alaska. Geophys. Res. Lett., 14: 143-146.

Isacks, B.L. and Barazangi, M., 1977. Geometry of Benioff zones: lateral segmentation and downward bending of the subducting lithosphere. Am. Geophys. Union, Maurice Ewing Series, 1: 99-114.

Isacks, B.L. and Molnar, P., 1969. Mantle earthquake mechanisms and the sinking of the lithosphere. Nature, 223: $1121-1124$.

Isacks, B.L. and Molnar, P., 1971. Distribution of stresses in the descending lithosphere from a global survey of focal-mechanisms solutions of mantle earthquakes. Rev. Geophys. Space Phys., 9: 103-174.

Isacks, B.L., Oliver, J. and Sykes, L.R., 1968. Seismology and the new global tectonics. J. Geophys. Res., 73: 5855-5899.

Isacks, B.L., Sykes, L.R. and Oliver, J., 1969. Focal mechanism of deep and shallow earthquakes in the Tonga-Kermadec region and the tectonics of island arcs. Geol. Soc. Am. Bull., 80: 1443-1470.

Isacks, B.L., Cardwell, R.K., Chatelain, J.L., Barazangi, M., Marthelot, J.M., Chim, D. and Loutat, R., 1979. Seismicity and tectonics of the central New Hebrides island arc. Am. Geophys. Union, Maurice Ewing Series, 4: 93-116.

Ishida, M., 1970. Seismicity and travel-time anomaly in and around Japan. Bull. Earth Res. Inst., 48: 1023-1051.

Ishikawa, Y., 1985. Double seismic zone beneath Kysushu, Japan. Zisin, 38: 265-269.

Jackson, J. and Fitch, T.J., 1979. Seismotectonic implications of relocated aftershock sequences in Iran and Turkey. Geophys. J. R. Astron. Soc., 57: 209-229.

Jackson, J. and Fitch, T., 1981. Basement faulting and the focal depths of the larger earthquakes in the Zagros mountains (Iran). Geophys. J. R. Astron. Soc., 64: 561-586.

Jacob, K.H. and Quittmeyer, R.L., 1979. The Makron region of Pakistan and Iran: trench-arc system with active plate subduction. In: Geodynamics of Pakistan.

Jarrard, R.D., 1986. Relations among subduction parameters. Rev. Geophys., 24: 217-284.

Johnson, T. and Molnar, P., 1972. Focal mechanism and plate tectonics of the southwest Pacific. J. Geophys. Res., 77: $5000-5032$.

Kanamori, H., 1970. Seismological evidence for lithospheric normal faulting: the Sanriku earthquake of 1933. Phys. Earth Planet. Inter., 4: 289-300.

Kanamori, H., 1977a. The energy release in great earthquakes. J. Geophys. Res., 82: 2981-2987.

Kanamori, H., 1977b. Seismic and aseismic slip along subduction zones and their tectonic implications. Am. Geophys. Union, Maurice Ewing Series, 1: 163-174.

Kanamori, H., 1983. Magnitude scale and quantification of earthquakes. Tectonophysics, 9: 185-199.

Kanamori, H., 1986. Rupture process of subduction-zone earthquakes. Ann. Rev. Earth Planet. Sci., 14: 293-322.

Kanamori, H. and McNally, K.C., 1982. Variable rupture mode of the subduction zone along the Ecuador-Colombia coast. Bull. Seismol. Soc. Am., 72: 1241-1253.

Kanamori, H. and Tsumura, K., 1971. Spatial distribution of earthquakes in the Kii Peninsula, Japan, south of the Median Tectonic line. Tectonophysics, 12: 327-342.

Katsumata, M. and Sykes, L.R., 1969. Seismicity and tectonics of the western Pacific: Izu-Mariana-Caroline and Ryuku-Taiwan Region. J. Geophys. Res., 74: 5923-5948.

Kawakatsu, H., 1985. Double seismic zones: A first order feature of plate tectonics. Ph.D. thesis, Stanford University, California.

Kawakatsu, H., 1986a. Double seismic zones: kinematics. J. Geophys. Res., 91: 4811-4825.

Kawakatsu, H., 1986b. Downdip tensional earthquakes beneath the Tonga arc: A double seismic zone? J. Geophys. Res., 91: 6432-6440.

Kawakatsu, H. and Seno, T., 1983. Triple seismic zone and the regional variation of seismicity along the northern Honshu arc. J. Geophys. Res., 88: 4215-4230.

Kelleher, J., 1972. Rupture zones of large South American earthquakes and some predictions. J. Geophys. Res., 77: 2087-2103

Kelleher, J., Savino, J., Rowlett, H. and McCann, W., 1974. Why and where great thrust earthquakes occur along island arcs. J. Geophys. Res., 79: 4889-4899.

Korrat, I. and Madariaga, R., 1986. Rupture of the Valparaiso (Chile) gap from 1971 to 1985. In: S. Das, J. Boatwright and C.H. Scholtz (Editors), Earthquake source mechanics. Am. Geophys. Union, pp. 247-258.

Langston, C.A. and Blum, D.E., 1977. The April 29, 1965 Puget Sound earthquake and the crustal and upper mantle structure of western Washington. Bull. Seismol. Soc. Am., 67: 693-711.

Lay, T. and Kanamori, H., 1980. Earthquake doublets in the Solomon Islands. Phys. Earth Planet. Inter., 21: 283-304.

LeDain, A.Y., Tapponier, P. and Molnar, P., 1984. Active faulting and tectonics of Burma and surrounding regions. $J$. Geophys. Res., 89: 453-472.

LeFevre, L.V. and McNally, K.C., 1985. Stress distribution and subduction of aseismic ridges in the Middle America subduction zone. J. Geophys. Res., 90: 4495-4510.

Malgrange, M. and Madariaga, R., 1983. Complex distribution of large thrust and normal fault earthquakes in the Chilean subduction zone. Geophys. J. R. Astron. Soc., 73: 489-506. 
Malgrange, M., Deschamps, A. and Madariaga, R., 1981. Thrust and extensional faulting under the Chilean coast, 1965, 1971 Aconcagua earthquakes. Geophys. J. R. Astron. Soc., 66: 313-331.

McCaffrey, R., Molnar, P., Roecker, S.W. and Joyodiwiryo, Y., 1985. Microearthquake seismicity and fault plane solutions related to arc-continent collision in the eastern Sunda arc, Indonesia. J. Geophys. Res., 90: 4511-4528.

McCann, W.R. and Sykes, L.R., 1984. Subduction of aseismic ridges beneath the Caribbean plate: implications for the tectonic and seismic potential of the northeastern Caribbean. J. Geophys. Res., 89: 4493-4519.

McCann, W.R., Nishenko, S.P., Sykes, L.R. and Krause, J., 1979. Seismic gaps and plate tectonics: seismic potential for major boundaries. Pageoph., 117: 1082-1147.

McKenzie, D., 1978. Active tectonics of the Alpine-Himalaya belt: the Aegean Sea and surrounding regions. Geophys. J. R. Astron. Soc., 55: 217-254.

McNally, K.C. and Minster, B., 1981. Nonuniform seismic slip rates along the Middle America trench. J. Geophys. Res., 86: 4949-4959.

McNally, K.C., Gonzalez-Ruiz, J.R. and Stolte, C., 1986. Seismogenesis of the 1985 Great $\left(M_{\mathrm{S}}=8.1\right)$ Michoacan, Mexico earthquake. Geophys. Res. Lett., 13: 585-588.

Michael-Leiba, M.O., 1984. The Banda Sea earthquake of 24 November 1983: evidence for intermediate-depth thrust faulting in the Benioff zone. Phys. Earth Planet. Inter., 36: 95-98.

Minster, J.B. and Jordan, T.H., 1978. Present day plate motions. J. Geophys. Res., 83: 5331-5354.

Mogi, K., 1973. Relationship between shallow and deep seismicity in the western Pacific region. Tectonophysics, 17: $1-22$.

Molnar, P. and Sykes, L.R., 1969. Tectonics of the Caribbean and Middle-America regions from focal mechanisms and seismicity. Geol. Soc. Am. Bull., 80: 1639-1684.

Molnar, P., Freedman, D. and Shih, J., 1979. Lengths of intermediate and deep seismic zones and temperatures in downgoing slabs of lithosphere. Geophys. J. R. Astron. Soc., 56: 41-54.

Moore, G.W., 1982. Plate tectonic map of the circum Pacific region, explanatory notes: Tulsa, OK. American Association of Petroleum Geologist, 14 pp.

Newcomb, K.R. and McCann, W.R., 1987. Seismic history and seismotectonics of the Sunda arc. J. Geophys. Res., 92: 421-440.

Nishimura, C., Wilson, D.S. and Hey, R.N., 1984. Pole of rotation analysis of present-day Juan de Fuca plate motion. J. Geophys. Res., 89: 10283-10290.

Oike, K., 1971. On the nature and occurrence of intermediate and deep earthquakes. 1. The world-wide distribution of the earthquake generating stress. Bull. Disas. Prev. Res. Inst., Kyoto U., 20: 145-182.

Osada, M. and Abe, K., 1981. Mechanism and tectonic implications of the great Banda Sea earthquake of November 4, 1963. Phys. Earth Planet. Inter., 25: 129-139.

Pascal, G., 1979. Seismotectonics of the Papua-New Guinea-Solomon Island region. Tectonophysics, 57: 7-34.
Pascal, G., Dubois, J., Barazangi, M., Isacks, B.L. and Oliver, J., 1973. Seismic velocity anomalies beneath the New Hebrides Island arc: evidence for a detached slab in the Upper-mantle. J. Geophys. Res., 78: 6998-7004.

Pascal, G., Isacks, B.L., Barazangi, M. and Dubois, R., 1978. Precise relocations of earthquakes and seismotectonics of the New Hebrides island arc. J. Geophys. Res., 83: 4957-4973.

Pennington, W.D., 1983. Role of shallow phase changes in the subduction of oceanic crust. Science, 220: 1045-1047.

Reid, I.D., 1976. The Rivera plate: A study in seismology and plate tectonics. Ph.D. thesis, University of California, San Diego.

Richter, C.F., 1958. Elementary Seismology. Freeman, San Francisco, CA, 768 pp.

Richter, F.M., 1979. Focal Mechanisms and seismic energy release of deep and intermediate earthquakes in the Tonga-Kermadec region and their bearing on the depth extent of mantle flow. J. Geophys. Res., 84: 6783-6795.

Ripper, I.D., 1974. Some earthquake focal mechanisms in the New Guinea-Solomon Island region, 1963-1968. Aust., Bur. Miner. Resour. Geol. Geophys. Rep. 178.

Ripper, I.D., 1975. Some earthquake focal mechanisms in the New Guinea-Solomon Island region, 1969-1971. Aust., Bur. Miner. Geol. Geophys. Resour. Rep. 192.

Ruff, L. and Kanamori, H., 1980. Seismicity and the subduction process. Phys. Earth Planet. Inter., 23: 240-252.

Ruff, L. and Kanamori, H., 1983. Seismic coupling and uncoupling at subduction zones. Tectonophysics, 99: 99-117.

Samowitz, I.R. and Forsyth, D.W., 1981. Double seismic zone beneath the Mariana Island arc. J. Geophys. Res., 86: 7013-7021.

Sasatani, T., 1976. Mechanisms of mantle earthquakes near the junction of the Kurile and northern Honshu arcs. J. Phys. Earth, 24: 341-354.

Schwartz, S. and Ruff, L.J., 1987. Asperity distribution and earthquake occurrence in the Southern Kurile Islands Arc. Phys. Earth Planet. Inter. (submitted).

Seno, T., 1977. The instantaneous rotation vector of the Philippine Sea plate relative to the Eurasian plate. Tectonophysics, 42: 209-226.

Seno, T. and Kurita, K., 1978. Focal mechanism and tectonics in the Taiwan-Philippine region. J. Phys. Earth, 26 Suppl., S249-S263.

Seno, T. and Pongswat, B., 1981. A triple-planed structure of seismicity and earthquake mechanisms at the subduction zone off Miyagi Prefecture, northern Honshu, Japan. Earth Planet. Sci. Lett., 55: 25-36.

Shiono, K., Mikumo, T. and Ishikawa, Y., 1980. Tectonics of the Kyushu-Ryukyu arc as evidenced from seismicity and focal mechanisms of shallow to intermediate depth earthquakes. J. Phys. Earth, 28: 17-43.

Silver, P.G. and Jordan, T.H., 1983. Total moment spectra of fourteen large earthquakes. J. Geophys. Res., 88: 3273-3293.

Singh, S.K., Astiz, L. and Havskov, J., 1981. Seismic gaps and recurrence periods of large earthquakes along the Mexican 
subduction zone: A reexamination. Bull. Seismol. Soc. Am., 71: $827-843$.

Singh, S.K., Suarez, G. and Dominguez, T., 1985. The Oaxaca, Mexico, earthquake of 1931: lithospheric normal faulting in the subducted Cocos plate. Nature, 317: 56-58.

Sleep, N.H., 1973. Teleseismic P-wave transmission through slabs. Bull. Seismol. Soc. Am., 63: 1349-1373.

Sleep, N.H., 1979. The double seismic zone in downgoing slabs and the viscosity of the mesosphere. J. Geophys. Res., 84: 4565-4571.

Soedarmo, P.D., 1973. Focal mechanisms of earthquake generating stress system in the Indonesia region. Presented at Symposium on Recent Crustal Movements, Bandung.

Spence, W., 1977. The Aleutian arc: Tectonic blocks, episodic subduction, strain diffusion, and magma generation. J. Geophys. Res., 82: 213-230.

Spence, W., 1986. The 1977 Sumba earthquake series: evidence for slab pull force acting at a subduction zone. J. Geophys. Res., 91: 7693-7701.

Spence, W., 1987. Slab pull and the seismotectonics of subducting lithosphere. Rev. Geophys., 25: 55-70.

Stauder, W., 1968. Tensional character of earthquake foci beneath the Aleutian trench with relation to sea-floor spreading. J. Geophys. Res., 73: 7693-7701.

Stauder, W., 1972. Fault motion and spatially bounded character of earthquakes in Amchitka Pass and the Delarof islands. J. Geophys. Res., 77: 2072-2080.

Stauder, W., 1973. Mechanisms and spatial distribution of the Chilean earthquakes with relation to subduction of the oceanic plate. J. Geophys. Res., 78: 5033-5061.

Stauder, W., 1975. Subduction of the Nazca plate under Peru as evidenced by focal mechanisms and seismicity. J. Geophys. Res., 80: 1053-1064.

Stauder, W. and Bollinger, G.A., 1966. The S-wave project for focal mechanism studies: Earthquakes of 1963. Bull. Seismol. Soc. Am., 56: 1363-1371.

Stauder, W. and Maulchin, L., 1976. Fault motion in the larger earthquakes of the Kurile-Kamchatka arc and the Kurile-Hokkaido corner. J. Geophys. Res., 81: 2297-2308.

Stein, S., Engeln, J.F., Wiens, D.A., Speed, R.C. and Fujita, K., 1982. Subduction, seismicity and tectonics in the Lesser Antilles arc. J. Geophys. Res., 87: 8642-8664.

Suzuki, S. and Motoya, Y., 1978. Microearthquake activity and a two-layered deep seismic zone in the Hokkaido region. Abstr. Progr. Seismol. Soc. Jpn., 1: 32 (in Japanese).

Sykes, L.R., 1966. Seismicity and deep structure of island arcs. J. Geophys. Res., 71: 2981-3006.

Sykes, L.R., 1971. Aftershock zones of great earthquakes, seismicity, gaps and earthquake prediction for Alaska and the Aleutians. J. Geophys. Res., 76: 8021-8041.

Sykes, L.R. and Quittmeyer, R.C., 1981. Repeat times of great earthquakes along simple plate boundaries. In: D.W. Simpson and P.G. Richards (Editors), Earthquake Prediction, an International Review. Am. Geophys. Union, Washington, DC, pp. 217-247.

Sykes, L.R., McCann, W.R. and Kafka, A.L., 1982. Motion of the Caribbean plate during the last 7 m.y. and implications for earlier Cenozoic movements. J. Geophys. Res., 87: 10656-10676.

Tsumara, K., 1973. Microearthquake activity in the Kanto district. In: Special Publication for the 50th Anniversary of the Great Kanto Earthquake, Earthquake Research Institute, Tokyo, pp. 67-87.

Uyeda, S. and Kanamori, H., 1979. Back-arc opening and the mode of subduction. J. Geophys. Res., 80: 1053-1064.

Vassiliou, M.S., 1984. The state of stress in subducting slabs as revealed by earthquakes analyzed by moment tensor inversion. Earth Planet. Sci. Lett., 69: 195-202.

Vassiliou, M.S., Hager, B.H. and Raefsky, A., 1984. The distribution of earthquakes with depth and stress in subducting slabs. J. Geodynamics, 1: 11-28.

Veith, K., 1974. The relationship of island and seismicity to plate tectonics, Ph.D. thesis, South Methodist University, Dallas, TX.

Veith, K., 1977. The nature of the dual zone of seismicity in the Kuriles arc. EOS, 58: 1232.

Vlaar, N.J. and Wortel, M.J.R,, 1976. Lithospheric aging, instability, and subduction. Tectonophysics, 32: 331-351.

Welc, J.L. and Lay, T., 1987. The source rupture process of the great Banda Sea earthquake of November 4, 1963. Phys. Earth Planet. Inter., 5: 242-254.

Wesnousky, S.G., Astiz, L. and Kanamori, H., 1986. Earthquake multiplets in the Southeastern Solomon Islands. Phys. Earth Planet. Inter., 44: 304-318.

Wickens, A.J. and Hodgson, J.H., 1967. Computer re-evaluation of earthquake mechanism solutions 1922-1962. Publ. Dom. Obs., Ottawa, 33: 1-560.

Wortel, R., 1982. Seismicity and rheology of subducted slabs. Nature, 296: 553-555.

Yamaoka, K., Fukao, Y. and Kumazawa, M., 1986. Spherical shell tectonics: effects of sphericity and inextensibility on the geometry of the descending lithosphere. Rev. Geophys., 24: $27-54$.

Yoshii, T., 1977. Structure of the Earth's crust and mantle in northeast Japan. Kagaku, 47: 170-176.

Yoshii, T., 1979. A detailed cross section of the deep seismic zone beneath Japan. Tectonophysics, 55: 349-360. 